\title{
MEASUREMENT OF THE RADIATIVE DECAY WIDTH \\ OF THE POSITIVE RHO MESON
}

\author{
by \\ Joey $\mathbf{W}$. Huston \\ Submitted in oPartial Fulfillment \\ of the \\ Requirements for the Degree \\ DOCTOR OF PHILOSOPHY \\ Department of Physics and Astronomy \\ The University of Rochester \\ Rochester, New York.
}


ACKNOWLEDGEMENTS

An experiment of this size could not have proceeded without the combined efforts of many individuals. The cast of characters includes: David Berg, Clark Chandlee, Selcuk Cihangir, Tom Ferbel, Terry Jensen, Fred tbbkowicz, Mike McLaughlin, Takayoshi Ohshima, Paul slattery and pat Thompson from the Oniversity of Rochester; Bruce arktop; steve Beppelmann, Terry Joyce, Marvin Marshak, Earl Patwowad, Reith Ruddick and Mike Shupe from the University of Mirrinesota; Joe Biel, Tom Droege, Alan Jonckheere, Peter Roehler and Charlie Nelson from Fermilab. Supporting roles were played by the National Science Foundation and the Department of Energy.

I am grateful for the advice and support I have received throughout my graduate career from professor Fred Lobkowicz.

I thankfully acknowledge the capable assistance of Professors Tom Ferbel, Paul slattery, Reith Ruddick, Marvin Markshak and Earl Peterson during the experimental run and during the data analysis. 
I would like to thank Terry Jensen and Pat Thompson for the detailed attention they have given to all facets of this experiment. Alan Jonckheere has my appreciation for steering the beam roughly to where it should have been and Charlie Nelson has my thanks for making sure that the. LAC worked once it got there.

I have greatly enjoyed my friendship with Takayoshi Ohshima from whom I have learned many things including the creative use of the English language.

I have had many interesting discussions with Marek zielinski and have learned reasonable pronounciation of several difficult polish words.

I have enjoyed working with my fellow.graduate students: David Berg, Clark Chandlee, Selcuk Cihangir, Bruce Collick, Mike McLaughlin and Steve Hepplelmann. I look forward to no longer being a graduate student.

I thank Mrs. Betty Cook for the counseling, advice and filling in of forms she has given to me throughout my studies.

I acknowledge the professional job done by John sheedy in making the figures for my thesis. 
My stay at Fermilab has been made more pleasant by my friendship with Selcuk Cihangir who is the only person I know who has been drafted into the Turkish army.

Finally, I would like to express my thanks to my family for their love and support: my sisters Kathy and Sherry, my brothers Jerry and Michael and, most of all, my mother and father, Mary and walter. 
ABSTRACT

We have investigated the coherent reaction $\pi^{+}+(A, Z)+\pi^{+} \pi^{0}+(A, Z)$ on three nuclear targets (carbon, copper and lead) at an incident momentum of $200 \mathrm{GeV}$. The $\pi^{+} \pi^{\circ}$ mass spectrum was found to be dominated by $\rho^{+}$ production. A fit was performed on the line shape of the $\rho^{+}$ and the values of $770 \mathrm{MeV}$ for the mass and $150 \mathrm{MeV}$ for the total width were found. The $t$ distributions were analyzed allowing a small amount of hadronic production to interfere with the dominant electromagnetic production of the $\rho^{+}$. A new value for the radiative width, $\Gamma\left(\rho^{+}+\pi^{+} \gamma\right)$, of $60 \pm 4 \mathrm{KeV}$ was extracted. This value is in reasonable agreement with that expected from VDM, SU(3) and non-relativistic quark model arguments. 
TABLE OF CONTENTS

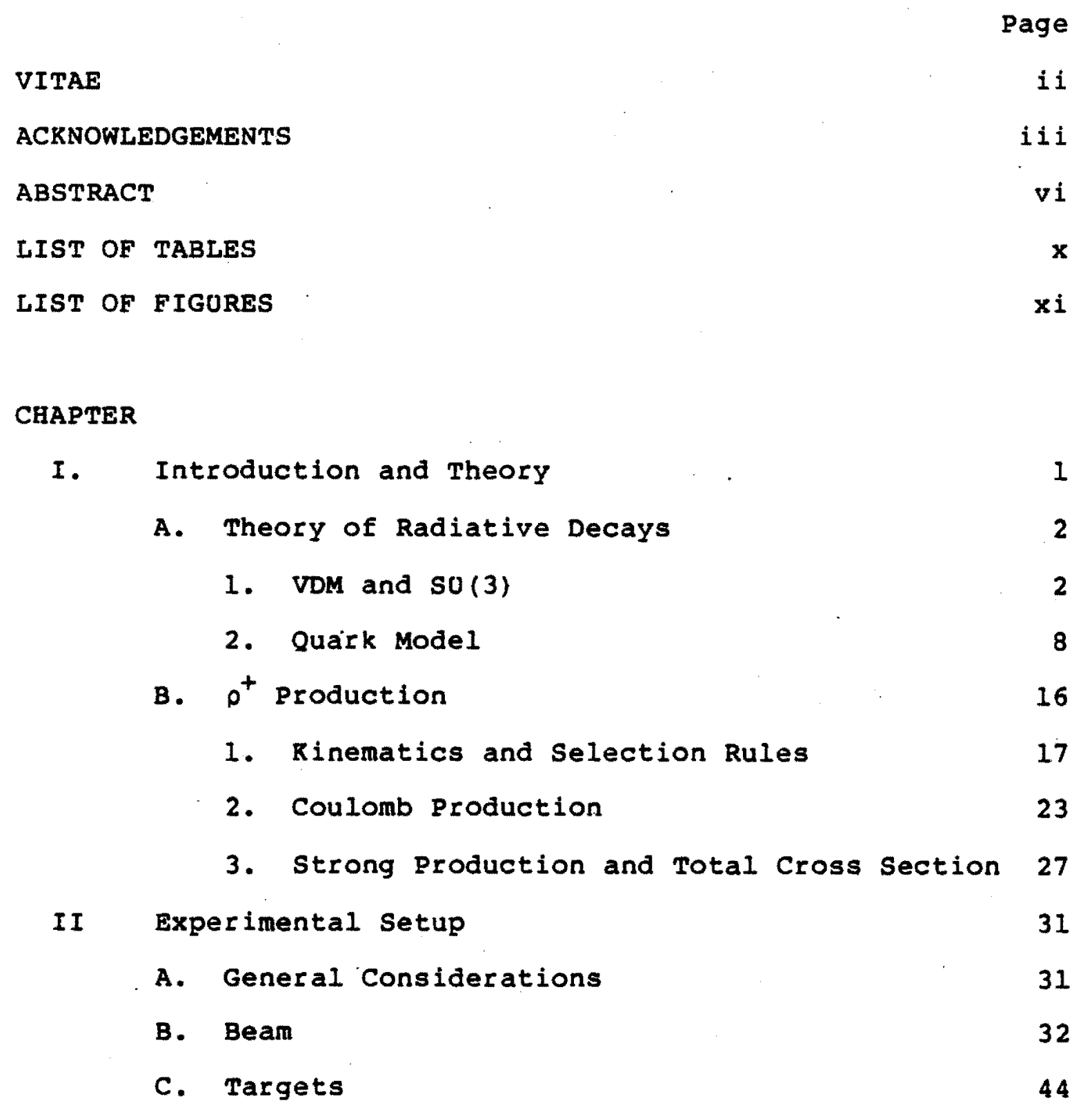


D. Particle Detection $\mathbf{4 4}$

1. Charged Particles 46

2. Photons 52

E. Experimental Trigger 64

F. Data Acquisition and Monitoring 76

III. Data Processing 80

A. Calibration and Alignment 80

1. Drift Chambers 80

2. Liquid Argon Calorimeter 86

B. Reconstruction 103

1. Charged Track Reconstruction 103

2. Photon Reconstruction 107

C. Run by Run Consistency 111

IV. Comparisons to Monte Carlo Model 115

A. Monte Carlo Model 115

B. Comparison of $\mathrm{K}^{+} \rightarrow \pi^{+} \pi^{\circ}$ DecaysMonte Carlo and Experiment 118

v. Normalization 138

A. Beam Normalization 139

B. Spectrometer Related Corrections 144

C. $\mathrm{R}^{+}$Decay Normalization 149

VI. $\rho^{+}$Data 154

A. Introduction 154

B. Data and Comparisons to Monte Carlo Model 155

c. Mass Fittings 166

D. Calculation of Cross Sections 274

E. Extraction of Radiative Width 176 
REFERENCES

APPENDIX A

203

APPENDIX B 


\section{LIST OF TABLES}

Table

Page

I Properties of the targets

II Resolution in $p_{t}$ for $\mathrm{R}^{+} \rightarrow \pi^{+} \pi^{\circ}$ decays

III Correction factors to beam normalization

a) effects independent of target

b) effects dependent on target

IV Correction factors to cross section

a) effects dependent on target

b) effects independent of target

V $\quad \mathbf{K}^{+} \rightarrow \pi^{+} \pi^{\circ}$ normalization factors

VI Results of mass fits

VII Summary of correction factors for $\rho^{+}$production 175

VIII Resolution for $\rho^{+} t$ fittings

IX Results of fits to t distributions

$x$ Integrated cross sections for $\rho^{+}$production 


\section{LIST OF FIGURES}

1. A graphical derivation of the ratio of the $\rho \pi \gamma$ and $\omega \pi y$ couplings using VDM and so(3) arguments. (Page 4)

2. A schematic depiction of the magnetic dipole transition responsible for the reaction $\rho^{+} \rightarrow \pi^{+} \gamma_{\text {. }}$ (Page 4)

3. a) A schematic depiction of the reaction $\pi^{+}+\gamma \rightarrow \rho^{+} \rightarrow \pi^{+} \pi^{\circ}$ where the $\gamma$ is from the coulomb field of a nucleus. b) A schematic depiction of the reaction $\pi^{+}+\omega^{0} \rightarrow p^{+} \rightarrow \pi^{+} \pi^{\circ}$ where the $\omega^{\circ}$ has been exchanged with a nucleus. (Page 4)

4. a) Diagram illustrating the kinematics for the reaction $\mathbf{a}+\mathbf{A}+\mathbf{a}^{\star}+\mathbf{A}$.

b) Diagram for the Coulomb production reaction $\pi^{+}+A \rightarrow \rho^{+}+A$ in the rest frame of the $\rho^{+}$(Gottfried-Jackson frame) :

c) Definition of the Gottfried-Jackson reference frame for the coherent process $\pi^{+}+A+\pi^{+} \pi^{\circ}+A$.

d) Definition of the helicity reference frame for the coherent process $\pi^{+}+A \rightarrow \pi^{+} \pi^{\circ}+A$. (Page 20)

5. Schematic of the beam transportation system for the $M-1$ beamline at Fermilab. The $Q i$ designate quadrupole magnets, the B $i$ dipole magnets, and $\mathrm{KIC}$ and $\mathrm{K} 2 \mathrm{C}$ the two 
primary Cerenkov counters. The position of the beryllium absorber is indicated(at $130 \mathrm{~m}$ ). (Page 34)

6. Particle beam fractions as a function of beryllium absorber length. The curves are the result of fits to the effective absorption cross section for each particle type. See Reference 33. (Page 37)

7. Particle beam fractions as a function of the production transverse momentum for two cases: no beryllium and $\mathbf{2 . 2}$ $m$ of beryllium absorber. See Reference 33 for more detail. (Page 38)

8: Detection efficiency of Cerenkov counter $\mathrm{Kl}$ as a function of the helium pressure. $K I$ is the coincidence (either 2-fold or 3-fold) of the signals from the Cerenkov counter and $B$ is the signal defining the beam. See the text in Chapter II for more detail. (Page 41)

9. Detection efficiency of Cerenkov counter $k 2$ as a function of helium pressure. (Page 42)

10. Detection efficiency as a function of helium pressure for the Cerenkov. counter R3. (Page 43)

11. Schematic of the E272 spectrometer. (Page 47)

12. Drift chamber read-out and calibration system. (Page 50)

13. Exploded view of the LAC. (Page 54) 
14. Schematic top and side views of the LAC and cryostat assembly. (Page 56)

15. Schematic of liquid argon purification system. (Page 58)

16. a) Block diagram of the electronics for one channel of the LAC.

b) Signal at point $c$ of the above diagram.

c) Read-out system for the LAC amplifiers. (Page 61)

17. Schematic of the scintillation counters used in the experiment. (Page 66)

18. Diagram of the logic used to define the Rho trigger. (Page 67)

19. Diagram of the trigger logic associated with the LAC. (Page 75)

20. Diagram of the on-line data acquisition system. (Page 78)

21. Plots of the calibration constants for two LRS-2770A TDC channels as a function of time. (Page 82)

22. Plots of TDC time versus projected beam position for the upsteam and downstream chambers. (Page 85)

23. Energy conversion factors for all strips in the front half of the LAC. (Page 90) 
24. Ratio of the energy determined by the LAC to the energy determined by the drift chambers and analyzing magnet for $50 \mathrm{GeV}$ electrons from the calibration run. The curve through the data is a Gaussian with an rms width of 1.468. The smaller Gaussian shows the drift chamber contribution to the resolution. (Page 92)

25. Difference of the position determined by the LAC from that determined by the drift chambers for electrons from the $50 \mathrm{GeV}$ calibration run. The curve through the data is a Gaussian with an rms width of $0.67 \mathrm{~mm}$. The smalier Gaussian shows the contribution of the drift chambers to the position resolution: (Page 92)

26. Energy measured by the LAC versus energy measured by the drift chambers and analyzing magnet for positrons from Re3 decays. (Page 94)

27. Energy resolution of the LAC (standard deviation squared) as a function of positron momentum for positrons from Re 3 decays. (Page 95)

28. Position resolution of the LAC for positrons from Re3 decays as a function of the positron momentum. (Page 98)

29. Electromagnetic and hadronic shower shapes in the LAC. (Page 99) 
30. a) Plot of the fraction of charged pions versus the ratio of the energy deposited in the LAC by the pions to the pion momentum measured by the drift chambers and analyzing magnet.

b) Plot of the fraction of electrons and charged pions versus the ratio of the energy deposited in the back of the LAC to the total energy deposited in the LAC by electrons and charged pions. (Page 101)

31. a) Resolution in $\mathrm{p}_{t}$ obtained for $\mathrm{R}^{+} \rightarrow \pi^{+} \pi^{\circ}$ decays versus relative $\dot{x}$ position(in the reconstruction program) of the LAC. The original LAC position and the position ultimately used in the analysis are shown by the arrows. b) Resolution in $p_{t}$ obtained for $\mathrm{K}^{+} \rightarrow \pi^{+} \pi^{\circ}$ decays versus relative $Y$ position of the LAC. The LAC has already been shifted to the $x$ position of the minimum in Figure 31a. (Page 104)

32. Scatterplot of the $X$ and $Y$ gamma energies for positrons from Ke3 decays. (Page 110)

33. Relative collected charge in the LAC (deduced from the $\pi^{\circ}$ mass) versus time. (Page 114)

34. Decay vertex distribution for $\mathrm{K}^{+} \rightarrow \pi^{+} \pi^{\circ}$ decay events and the Monte Carlo prediction. The arrows indicate the region used for normalization. (Page 120)

35. Decay angle distribution in the helicity frame for 
$\mathrm{K}^{+} \rightarrow \pi^{+} \pi^{\circ}$ decays. The smooth curve is a Monte Carlo prediction assuming an isotropic decay of the $\mathrm{K}^{+}$. (Page 122)

36. Distribution of the transverse distance of the $\pi^{+}$from beam center for $\mathrm{K}^{+} \rightarrow \pi^{+} \pi^{\circ}$ decays and the Monte carlo comparison. (Page 122)

37. Asymmetry distribution and Monte Carlo comparison for the two photons from the $\pi^{\circ}$ in $\mathrm{K}^{+}+\pi^{+} \pi^{\circ}$ decays. (Page 124)

38. Energy distribution for $\pi^{\circ}$ 's from $\mathrm{R}^{+} \rightarrow \pi^{+} \pi^{\circ}$ decays and the Monte Carlo comparison. (Page 124)

39. a) Separation $\left(R=r\left(X^{2}+Y^{2}\right)\right)$ in the LAC of the two photons from the $\pi^{\circ}$ in $\mathrm{K}^{+}+\pi^{+} \pi^{\circ}$ decays. The smooth curve is the Monte Carlo prediction.

b) Separation of the two photons in one view ( $X$ or $Y$ ) and the Monte Carlo prediction. (Page 125)

40. Energy resolution of LAC (standard deviation squared) for Monte Carlo Re3 events as a function of positron momentum. For comparison the data resolution function is plotted as a dashed line. (Page 127)

41. Position resolution of the LAC for positrons from data Ke3 decays as a function of positron momentum. For comparison the position resolution predicted by the Monte Carlo is plotted as a smooth curve. (Page 128) 
42. Two photon mass for $\mathrm{K}^{+} \rightarrow \pi^{+} \pi^{\circ}$ decays and Monte Carlo comparison. (Page 130)

43. $\pi^{\circ}$ mass resolution versus $\pi^{0}$ energy for $K^{+}+\pi^{+}, 0$ decays. The Monte Carlo prediction is plotted as a smooth curve and the prediction based on the position and energy resolutions obtained with $\mathrm{Ke} 3$ decays is plotted as a dashed curve. The events have been restricted to asymmetries less than 0.33 . (Page 132)

44. $\pi^{+} \pi^{\circ}$ mass distribution from $\mathrm{R}^{+} \rightarrow \pi^{+} \pi^{\circ}$ decays and Monte Carlo comparison. (Page 133)

45. Total energy distribution for $\mathrm{K}^{+} \rightarrow \pi^{+} \pi^{\circ}$ decays and the Monte Carlo comparison. (Page 133)

46. $t$ aistributions observed for the three targetsland target empty) for $\mathrm{R}^{+} \rightarrow \pi^{+} \pi^{\circ}$ decays and the Monte Carlo predictions. (Page 135)

47. a) $\pi^{+} \pi^{\circ}$ mass distribution for the coherent process $\pi^{+} \mathrm{Pb} \rightarrow \pi^{+} \pi^{\circ} \mathrm{Pb}$.

b) $\pi^{+} \pi^{\circ}$ mass distribution for the coherent process $\pi^{+} \mathrm{Cu} \rightarrow \pi^{+} \pi^{\circ} \mathrm{Cu}$.

c) $\pi^{+} \pi^{\circ}$ mass distribution for the coherent process $\pi^{+} \mathrm{C} \rightarrow \pi^{+} \pi^{\circ} \mathrm{C}$.

The arrows indicate the limits used to define the $\rho^{+}$ signal. (Page 154) 
48. a) Reconstructed interaction vertex distribution for $\rho^{+}$ events. The larger histogram is for the lead target. The smaller histogram shows the target empty background scaled to the lead target beam count.

b) Reconstructed interaction vertex distribution for the lead target, now with target empty subtracted (and restricted to $t<.002 \mathrm{GeV}^{2}$, and the Monte carlo prediction. The arrows indicate the interaction vertex restriction used in the analysis. (Page 157)

49. a) Acceptance corrected distribution of the decay angle $\theta$ of the $\pi^{+}$in the Gottfried-Jackson frame for the coherent process $\pi^{+} \mathrm{Pb} \rightarrow \pi^{+} \pi^{\circ} \mathrm{Pb}$. The smooth curve shows the $\sin ^{2} \theta$ shape expected for coherently produced $\rho^{+}$mesons.

b) The combined geometric acceptance and reconstruction efficiency as a function of $\cos \theta_{G J} \cdot($ Page 159)

50. a), b) The distribution of the decay angle $\phi$ of the $\pi^{+}$ in the Gottfried-Jackson frame for the coherent process $\pi^{+} \mathrm{Pb} \rightarrow \pi^{+} \pi^{\circ} \mathrm{Pb}$. The top curve has a $t$ cut of $t<.002\left(\mathrm{GeV}^{2}\right)$ and the bottom curve has a $t$ cut of $.0005<t<.002\left(\mathrm{GeV}^{2}\right)$. (Page 161)

51. a) Distribution of total reconstructed energy for $0^{+}$ events on the lead target. The shaded region shows the effect of imposing a $t<.002 \mathrm{GeV}^{2}$ cut.

b) The shaded region above now compared with the Monte Carlo prediction. The two arrows indicate the 
restriction on total energy applied in the analysis. (Page 163)

52. a) Two photon mass distribution for $\rho^{+}$events on the lead target with Monte Carlo prediction. The two arrows indicate the $\pi^{\circ}$ mass cut used in the analysis.

b) Asymmetry distribution of the two photons for the events above that pass the $\pi^{\circ}$ mass cut. The Monte Carlo prediction is shown as a smooth curve. (Page 164)

53. $\pi^{\circ}$ energy distribution $p^{+}$events on the lead target and the Monte Carlo prediction. (Page 165)

54. Acceptance corrected $\pi^{+} \pi^{0}$ mass spectrum for the process $\pi^{+} \mathrm{Pb} \rightarrow \pi^{+} \pi^{\circ} \mathrm{Pb}$ for $t<.002 \mathrm{GeV}^{2}$. The acceptance as a function of mass is plotted above the data. The smooth curve through the data describes the best fits to the mass shape. (Page 167)

55. Acceptance corrected $\pi^{+} \pi^{\circ}$ mass spectrum for the process $\pi^{+} \mathrm{Cu} \rightarrow \pi^{+} \pi^{\circ} \mathrm{Cu}$. (Page 168)

56. Differential cross sections $(d \sigma / d t)$ for $\rho^{+}$production on the lead, copper and carbon targets. The smooth curves indicate the results of fits on the individual targets. (Page 177)

57. Definition of the regions over which the coulombic form factor is calculated. (Page 179) 
58. a) Schematic representation of one cell of LAC. b) Current waveform for the case of uniform ionization. c) Charge waveform for the case of uniform ionization. (Page 204)

59. Ratio of charge collected to total charge expected as a function of the electric field. The operating point is shown by the arrow. (Page 206) 


\section{Chapter I}

Introduction and Theory

In this thesis we report our measurement of the radiative width $\Gamma\left(\rho^{+}+\pi^{+} \gamma\right)$. The measurement was carried out through a study of the coherent reaction

$$
\pi^{+}+(A, Z)+\rho^{+}+(A, Z)
$$

where $(A, Z)$ represents targets of different atomic mass and atomic number. The theoretical framework needed to understand the above reaction is discussed in this chapter. In Chapter II the experimental apparatus is described and Chapter III details the reconstruction process and the resolution obtained in the different elements of the spectrometer. The Monte Carlo model used to simulate the experiment is described in Chapter IV, and in Chapter $V$ the normalization of the production cross section is described. In Chapter VI we present our analysis of Reaction (1.1) and extract a value for $\Gamma\left(\rho^{+}+\pi^{+} Y\right)$. 
A. Theory of Radiative Decays

Rates for radiative transitions of the form

$$
V \rightarrow P+Y
$$

can be understood theoretically by application of the vector Dominance Model(VDM), su(3) flavor symmetry, and the quark model. In the above reaction, $V$ stands for vector meson and P for pseudoscalar meson. Attempts to understand radiative transitions of this type will be described in this chapter.

1. VDM and SU(3)

In the Vector Dominance Model, the vector mesons $\rho, \omega$, and $\phi$ couple to the same electromagnetic current as the photon. [1] using the vector meson-photon couplings,the electromagnetic current $j_{\mu}$ can be written

$$
j_{\mu}(x)=\frac{e m_{\rho}^{2}}{2 \gamma_{\rho}} \rho_{\mu}(x)+\frac{e m_{\omega}^{2} \sin \theta_{v}}{2 \sqrt{3} \gamma_{Y}} \omega_{\mu}(x)+\frac{e m_{\phi}{ }^{2} \cos \theta_{v}}{2 \sqrt{3} \gamma_{Y}} \phi_{\mu}(x)
$$

$\rho_{\mu}(x), w_{\mu}(x)$, and $\phi_{\mu}(x)$ are the field operators for the neutral $\rho$ meson, $w$ meson, and $\phi$ meson respectively. The vector meson couplings are defined by 


$$
\begin{aligned}
& g_{\rho Y}=\frac{1}{\gamma_{\rho}} \\
& g_{\omega \gamma}=\frac{\sin \theta_{v}}{\sqrt{3} \gamma_{Y}} \\
& g_{\phi Y}=\frac{\cos \theta_{v}}{\sqrt{3} \gamma_{Y}}
\end{aligned}
$$

In the limit of $s u(3)$ symmetry, $\gamma_{P}=\gamma_{Y}$. The angle $\theta_{V}$ describes the mixing of the $\omega-\phi$ system. The physical $\omega$ and $\phi$ states are defined in terms of the octet $\left(\omega_{8}\right)$ and singlet $\left(\omega_{1}\right)$ members of the $I=Y=0$ members of the $s 0(3)$ representation. [2]

$$
\begin{aligned}
& \omega=\omega_{8} \sin \theta_{v}+\omega_{1} \cos \theta_{v} \\
& \phi=\omega_{8} \cos ^{\theta} v-\omega_{1} \sin \theta_{v}
\end{aligned}
$$

For the case of "ideal mixing", $\theta_{v}=\tan ^{-1}(1 / / 2)$. Ideal mixing leaves the $\phi$ as a pure ss state and leaves the $\omega$ with no strange component. For ideal mixing the coupling constants are in the ratios

$$
g_{\rho \gamma}^{2}: g_{\omega \gamma}^{2}: g_{\phi \gamma}^{2}=9: 1: 2
$$

Using these couplings the widths for various radiative decays can be related. (See Figure 1) For example, ignoring phase space factors, 


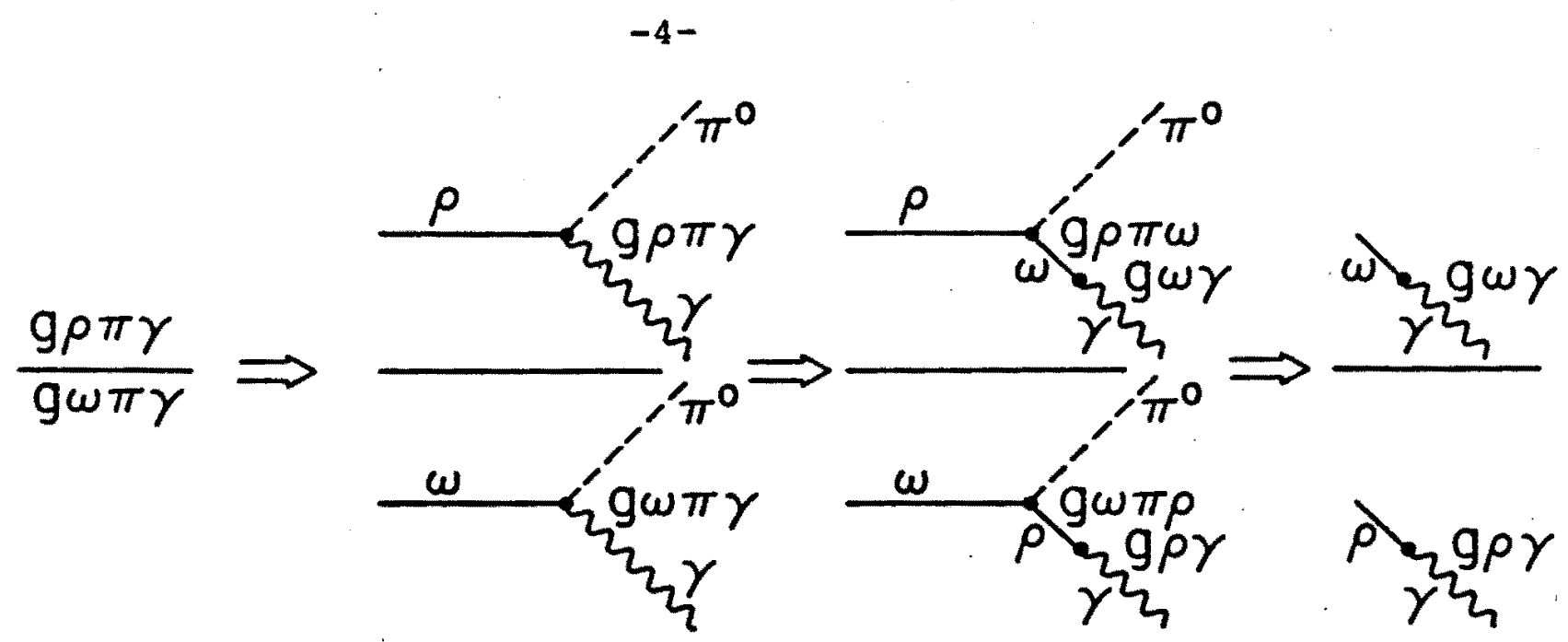

Figure 1

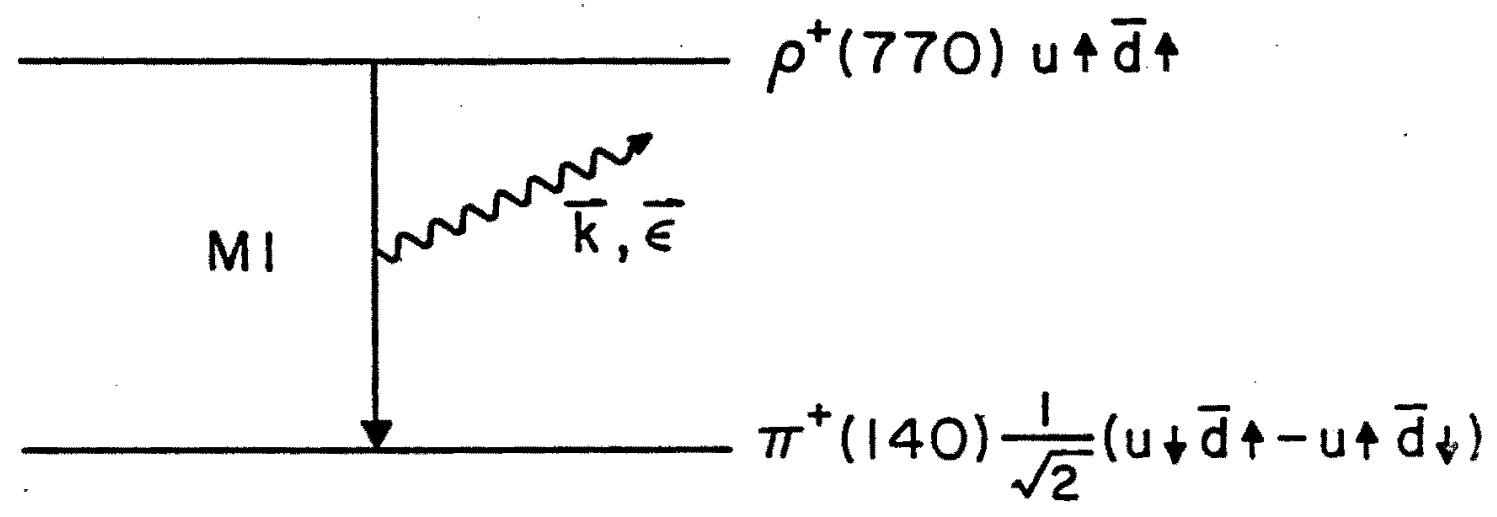

Figure 2
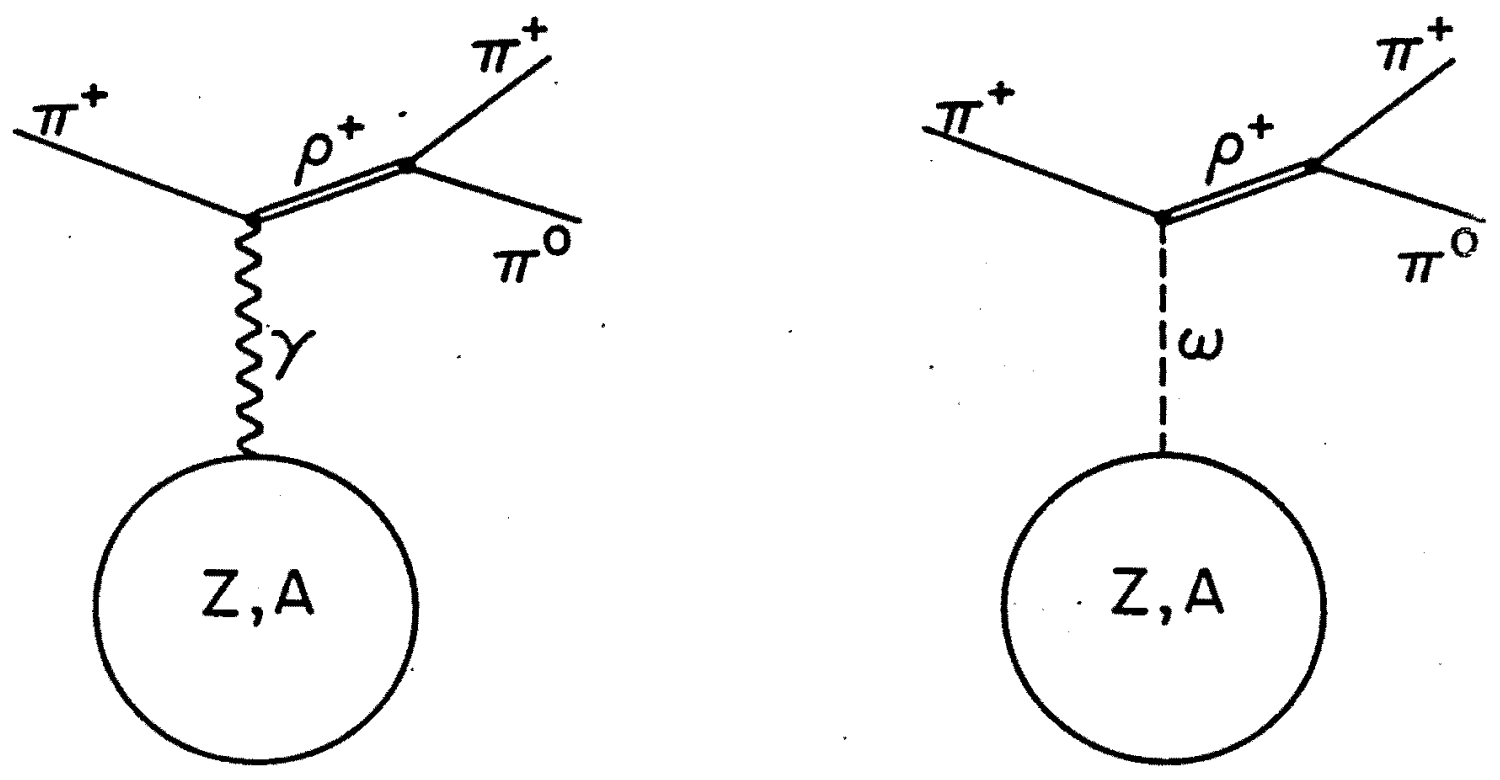

Figure 3 


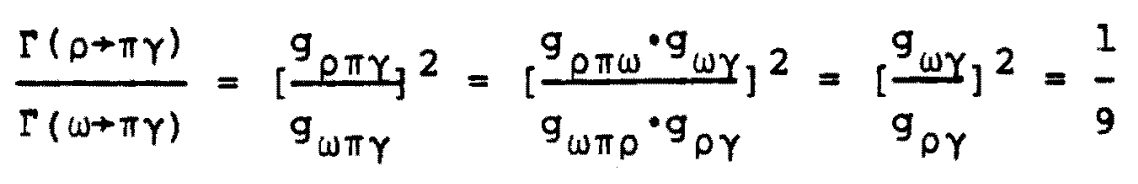

su(3) invariance of the strong and electromagnetic interactions leads to the following relations among the processes of the form $V+P+Y$ [3]

$$
\frac{g}{3}=g_{\rho} o^{\circ} o_{\gamma}=g_{\rho} \pm^{ \pm}{ }^{ \pm}=\frac{1}{\sqrt{3}} g_{\omega_{8} \pi^{\circ} \gamma}
$$

Further relations are possible by using u-spin. The photon is a 0-spin scalar and thus conserves 0-spin. within a su(3) multiplet, conservation of u-spin means conservation of charge.

$$
g_{\omega_{1} \pi} o_{\gamma}=\frac{2}{3} g_{1}
$$

In the above relations, $g$ describes the vector octet-pseudoscalar octet- $\gamma$ coupling $\left(V_{8} P_{8} \gamma\right)$ and $g_{1}$ describes the vector singlet-pseudoscalar octet- $\gamma \operatorname{coupling}\left(V_{1} P_{8} \gamma\right)$. Note that many more relationships among so(3) processes are provided by so(3) symmetry than shown above. I have merely 
mentioned the ones germane to a comparison of the $\rho^{+} \rightarrow \pi^{+} \gamma$ and $\omega^{\circ} \rightarrow \pi^{\circ} \gamma$ decays. A comparison is usually made to the $\omega^{\circ}$ radiative width since it is one of the largest and easiest to measure.

In terms of these couplings the radiative widths are given by the formula [3]

$$
\begin{aligned}
& \Gamma(\mathrm{V}+\mathrm{P} Y)=\frac{4}{3} \alpha \frac{\mathrm{k}^{3}}{\mathrm{~m}_{\pi}^{2}}\left|g_{V P \gamma}\right|^{2} \\
& \text { where } k=\frac{\left(m_{V}{ }^{2}-m_{P}{ }^{2}\right)}{2 m_{V}}
\end{aligned}
$$

By again assuming ideal mixing, the $\omega \pi r$ and $\phi \pi \gamma$ couplings can be written

$$
\begin{aligned}
& g_{\omega \pi \gamma}=\frac{1}{3}\left(2 g_{1}+g\right) \\
& g_{\phi \pi \gamma}=\frac{\sqrt{2}}{3}\left(g-g_{1}\right)
\end{aligned}
$$

By invoking nonet symmetry $\left(g=g_{1}\right.$, suggested by the smallness of the $\phi \rightarrow \pi r$ decay), the above relations simplify to

$$
\begin{aligned}
& g_{\omega \pi \gamma}=g \\
& g_{\phi \pi \gamma}=0
\end{aligned}
$$


Thus

$$
\frac{\Gamma\left(\rho^{+}+\pi^{+} \gamma\right)}{\Gamma\left(\omega^{\circ}+\pi^{\circ} \gamma\right)}=\left[\frac{\left(m_{\rho}^{2}-m_{\pi}{ }^{2}\right) / 2 m_{\rho}}{\left(m_{\omega}{ }^{2}-m_{\pi}{ }^{2}\right) / 2 m_{\omega}}\right]^{3} \cdot \frac{1}{9}=.95 \cdot \frac{1}{9}=.106
$$

Note that since the $\rho$ and $\omega$ mesons are close in mass, the phase space factor contributes little to the ratio. The value of $1 / 9$ was also found earlier using VDM arguments.

The $\rho \pi \gamma$ coupling can also be related to the $\pi^{\circ} \gamma \gamma$ coupling, again using vector dominance. [5] If one photon from the $\pi^{\circ}$ couples to the $\rho^{\circ}$, isospin requires the other to couple to the isoscalar vector meson( $\omega$ or $\phi)$. Thus, we can write

$$
g_{\pi}{ }^{\circ} \gamma \gamma=\frac{e}{2 \gamma_{\rho}} g_{\pi \rho \gamma}+\frac{e}{2 \sqrt{ } 3 \gamma Y}\left(\sin \theta_{v} g_{\pi \omega \gamma}+\cos \theta_{v} g_{\pi \phi \gamma}\right)
$$

Assuming ideal mixing and nonet symmetry and using the relations $(1.8),(1.12 a)$, and $(1.12 b)$, we have

$$
g_{\pi}^{\circ}{ }_{Y \gamma}=\frac{e}{3 \gamma_{\rho}} g
$$

The $\pi^{\circ} \rightarrow \gamma Y$ width can be written 


$$
\Gamma\left(\pi^{\circ}+\gamma \gamma\right)=\frac{\alpha_{m}}{4}\left|g_{\pi}{ }^{\circ} \gamma \gamma\right|^{2}
$$

so using (1.10a) we have.

$$
\frac{\Gamma\left(\rho^{+}+\pi^{+} \gamma\right)}{\Gamma\left(\pi^{\circ} \rightarrow \gamma \gamma\right)} \simeq \frac{16}{3}\left(\frac{k}{m_{\pi}}\right)^{3} \frac{\gamma_{\rho}^{2}}{4 \pi \alpha}
$$

where $k$ has the same definition as before. The process $\rho^{0}+e^{+} e^{-}$can be used measure $\gamma_{\rho}^{2} / 4 \pi$. The width can be written [6]

$$
\Gamma\left(\rho^{\circ}+e^{+} e^{-}\right)=\frac{\pi \alpha^{2}}{3} m_{\rho} \frac{1}{\gamma_{\rho}^{2}}\left(1+\frac{2 m_{e}^{2}}{m_{\rho}^{2}}\right)\left(1-\frac{4 m_{e}^{2}}{m_{\rho}^{2}}\right) 1 / 2
$$

In the Particle Data Book, $\Gamma\left(\rho^{0+e^{+}} e^{-}\right)=6.58 \pm 0.69 \mathrm{kev}$. Therefore $\gamma_{\rho}^{2 / 4 \pi}=0.52$. Also in the Particle Data Book $\pi^{\circ} \rightarrow Y Y=7.86 \pm 0.55$ ev. [7] Substituting these numbers in (1.17), we obtain for $\Gamma\left(\rho^{+} \rightarrow \pi^{+} \gamma\right)$ a value of $59 \pm 8 \mathrm{KeV}$.

\section{A. 2 Quark Model}

In the so(3) quark model, mesons consist of bound states of quarks and anti-quarks. [8] Thus both the $\rho^{+}$and $\pi^{+}$mesons can be considered to be composed of an up(charge +2/3, spin 1/2) and an antidown quark (charge $1 / 3, \operatorname{spin} 1 / 2$ ). 
The two mesons have the same quark content and are distinguised by their spin wave functions. In the $\rho^{+}$, the two spins are parallel, leading to a total spin of 1 . In the $\pi^{+}$, the two spins are anti-parallel for a composite spin of 0 . As shown in Figure 2, the process $\rho^{+}+\pi^{+} \gamma$ can be considered as a spin flip or magnetic dipole transition. Magnetic dipole, or Ml, transitions have a long history of application in atomic physics [9] and were applied to radiative decays of vector mesons at an early stage of development of the quark model. [10] The following section outlines a treatment of the Ml transition in the non-relativistic quark model.

The s-matrix in a relativistic, covariant treatment of the decay $v \rightarrow p+r$ can be written.

$$
s_{f i}=-i(2 \pi)^{4} \delta^{4}\left(k+k_{p}-k_{V}\right) T_{f i}
$$

$k$ is the 4-momentum of the photon, $k_{P}$ the 4-momentum of the pseudoscalar meson, and $k_{v}$ the 4-momentum of the vector meson. The matrix element $T_{f i}$ has the following relationship with the invariant Feynman amplitude $m_{f i}$ 


$$
T_{f i}=\frac{m_{f i}}{\left(2 k 2 E_{v} 2 E_{p}\right)^{1 / 2}}
$$

The decay rate $\Gamma$ in the rest frame of $V$ can be constructed by squaring $\mathbf{T}_{f i}$ and summing over final states

$$
\Gamma=(2 \pi)^{4} \int \frac{d^{3} k}{(2 \pi)^{3}} \frac{d^{3} k_{P}}{(2 \pi)^{3}} \delta^{3}\left(\vec{k}_{P}+\vec{k}\right) \delta\left(m_{V}-E_{P}-k\right) \cdot\left[\frac{1}{2 m_{V}} \frac{1}{2 k} \frac{1}{2 E_{P}}\left|m_{f i}\right|^{2}\right.
$$

In a non-relativistic treatment the bound state wave funtions are normalized in a non-covariant way by $\left\langle\Psi_{f} \mid \Psi_{i}\right\rangle=\delta_{f i}$ and the amplitude for the reaction $|i>\rightarrow| f\rangle+\gamma$ is denoted by $e M / f(2 k)$. If we now make an approximation and set this amplitude equal to $T_{E i}$

$$
T_{f i} \simeq \frac{e M}{\sqrt{(2 k)}}=\frac{m_{f i}}{\left(2 k 2 E_{v^{2}} E_{p}\right)^{1 / 2}}
$$

or

$$
m_{f i} \simeq\left(2 m_{V} 2 E_{p}\right)^{1 / 2} \mathrm{eM}
$$

Substituting into Equation (1.21)

$$
I(V \rightarrow P, Y)=\frac{e^{2}}{2 \pi} \frac{k E_{P}}{m_{V}} \int \frac{d \Omega}{4 \pi} \sum|M|^{2}
$$


where

$$
E_{P}=\left(k^{2}+m_{P}^{2}\right)^{1 / 2}=\frac{\left(m_{v}{ }^{2}+m_{p}{ }^{2}\right)}{2 m_{v}}
$$

and

$$
k=\frac{\left(m_{v}^{2}-m_{p}^{2}\right)}{2 m_{v}}
$$

In the long wavelength limit the matrix element $M$ can be written

$$
e M=\mu_{q} e_{q} \vec{\sigma} \cdot(\vec{k} \times \vec{\varepsilon})+\underset{\text { terms which vanish if the }}{\text { parity remains unchanged }}
$$

Now expanding

$$
\sum|\vec{\sigma} \cdot \vec{k} \times \vec{\varepsilon}|^{2}=\sigma^{2}\left[k^{2} \varepsilon^{2}=2 k^{2} \sigma^{2}\right.
$$

and substituting(and averaging over initial spins)

$$
\Gamma(V \rightarrow P \gamma)=\frac{2}{3}-\alpha k^{3}\left(\frac{{ }_{p}}{m_{v}}\right) \quad\left[|<v| \frac{{ }_{q} e_{q} \sigma_{q} \sigma_{q}}{e}|P>|^{2}\right.
$$


The usual assumption made at this point is that the $V$ and $P$ spatial wave funtions have a perfect overlap(although attempts have been made to allow the overlap integral to be less than unity[12]). Setting $\mu_{q}=\mu_{p}$ and evaluating the above matrix elements we have for the decays $\omega^{\circ} \rightarrow \pi^{\circ} \gamma$ and $\rho^{+} \rightarrow \pi^{+} \gamma$

$$
\begin{aligned}
& \Gamma\left(\omega^{0} \rightarrow \pi^{\circ} \gamma\right)=\frac{4}{3} \alpha k_{\omega}^{3} \mu_{p} 2\left(\frac{E_{\pi}}{m_{\omega}}\right) \\
& \Gamma\left(\rho^{+} \rightarrow \pi^{+} \gamma\right)=\frac{4}{27} \alpha k_{\rho}^{2} \mu_{p}^{2}\left(\frac{E_{\pi}}{m_{\rho}}\right)
\end{aligned}
$$

Substituting the values $k_{\omega}=.379 \mathrm{Gev}$ and $k_{\rho}=.372 \mathrm{Gev}$ and $\mu_{\mathrm{p}}=.00149 \mathrm{MeV}^{-1}$ we have

$$
\begin{aligned}
& \left.\Gamma\left(\omega^{\circ} \rightarrow \pi^{\circ} \gamma\right)=1.17 \underset{m_{\omega}}{E_{\pi}}\right) \quad(\mathrm{MeV}) \\
& \Gamma\left(\rho^{+} \rightarrow \pi^{+} \gamma\right)=0.123\left(\frac{E_{\pi}}{\mathrm{m}_{\rho}} \quad(\mathrm{MeV})\right.
\end{aligned}
$$

Alternatively, we can allow the quark magnetic moments to be determined by the magnetic moments of the proton, neutron and lambda. The quark magnetic moment can be written 


$$
\mu_{q}=\frac{g_{q}}{2 m_{q}}
$$

where $g_{q}$ is the gyromagnetic ratio of the quark and $m_{q}$ is the mass of the quark. Assuming pointlike behavior $\left(g_{q}=1\right)$, we can obtain(from the proton, neutron and lambda moments)

$$
\begin{aligned}
& \mu_{u}=\frac{1}{2 m_{u}}=\frac{1}{2(338)}=.00148 \mathrm{MeV}^{-1} \\
& \mu_{d}=\frac{1}{2 m_{d}}=\frac{1}{2(322)}=.00155 \mathrm{MeV}^{-1}
\end{aligned}
$$

The radiative widths of the $\omega^{\circ}$ and $\rho^{+}$then can be written

$$
\begin{aligned}
& \left.\Gamma\left(\omega^{\circ} \rightarrow \pi^{\circ} \gamma\right)=\frac{4}{3} k_{\omega}{ }^{3} \underset{3}{\left(-\mu_{u}\right.}+\frac{1}{3} \mu_{d}\right)^{2} \frac{E_{\pi}}{m_{\omega}} \\
& \left.\Gamma\left(\rho^{+} \rightarrow \pi^{+} \gamma\right)=\frac{4}{3}-\alpha k_{\rho}^{3 \cdot} \underset{3}{(2} \mu_{u^{-}}-\frac{1}{3} \mu_{d}\right) 2 \frac{E_{\pi}}{m_{\rho}}
\end{aligned}
$$

or , substituting,

$$
\begin{aligned}
& \Gamma\left(\omega^{\circ} \rightarrow \pi^{\circ} \gamma\right)=1.198 \frac{E_{\pi}}{m_{\omega}}(\mathrm{MeV}) \\
& \Gamma\left(\rho^{+} \rightarrow \pi^{+} \gamma\right)=0.111 \frac{\mathrm{E}_{\pi}}{\mathrm{m}_{\rho}} \quad(\mathrm{MeV})
\end{aligned}
$$


Now the phase space factor $E_{\mathrm{p}} / \mathrm{mV}_{\mathrm{V}}$ was introduced earlier in Equation (1.24) when the non-relativistic approximation was made. It was pointed out in the original calculation [10] that this factor would be replaced by unity if the relativistically invariant form of the $V_{Y}$ vertex was used. Setting $E_{\pi} / m_{\rho}$ and $E_{\pi} / m_{\omega}$ to. $I$ we arrive at a value for $\Gamma\left(\omega^{\circ} \rightarrow \pi^{\circ} \gamma\right)$ of $1.17 \mathrm{MeV}$ and for $\Gamma\left(\rho^{+} \rightarrow \pi^{+} \gamma\right.$ of $123 \mathrm{KeV}$ for the case $\mu_{\mathrm{q}}=\mu_{\mathrm{p}}$. If we allow symmetry breaking $\left(m_{\mathrm{u}} \neq \mathrm{m}_{\mathrm{d}}\right)$, we obtain $\Gamma\left(\omega^{\circ} \rightarrow \pi^{\circ} \gamma\right)=1.198 \mathrm{MeV}$ and $\Gamma\left(\rho^{+} \rightarrow \pi^{+} \gamma\right)=111 \mathrm{KeV}$. The above values for $\Gamma\left(\omega^{\circ}+\pi^{\circ} \gamma\right)$ are close to the value observed in early experiments. [13] As pointed out by 0'Donnell in Reference 3. the non-relativistic calculation neglects any recoil $(k=0)$ and it should hold only if $\mathbf{m}_{\pi} \approx \mathbf{m}_{\omega}$ or equivalently $E_{\pi} / m_{V} \approx 1$. osing physical masses for $\omega^{\circ} \rightarrow \pi^{\circ} \gamma$ and $\rho^{+} \rightarrow \pi^{+} \gamma, E_{\pi} / m_{V}(\rho$ or $\omega)=0.52$. Thus the non-relativistic quark model predictions of $\Gamma\left(\rho^{+}+\pi^{+} \gamma\right)$ and $\Gamma\left(\omega^{\circ} \rightarrow \pi^{\circ} \gamma\right)$ drop by a factor of 2 if the physical masses are used.

Some of the ambiguity is removed if a comparison of the two widths $\Gamma\left(\omega^{\circ}+\pi^{\circ} \gamma\right)$ and $\Gamma\left(\rho^{+}+\pi^{+} \gamma\right)$ is made. Since the two masses are very similar, any phase space factor must be very similar. The ratio is

$$
\frac{\Gamma\left(\rho^{+}+\pi^{+} \gamma\right)}{\Gamma\left(\omega^{0} \rightarrow \pi^{\circ} \gamma\right)}=0.105
$$




$$
\frac{\Gamma\left(p^{+}+\pi^{+} \gamma\right)}{\Gamma\left(\omega^{\circ}+\pi^{\circ} \gamma\right)}=0.093
$$

for symmetry breaking allowed. Using one of the latest estimates for $\Gamma\left(\omega^{\circ}+\pi^{\circ} \gamma\right)$ of $789 \pm 82 \mathrm{KeV}$ [14], we obtain an estimate for $\Gamma\left(\rho^{+} \rightarrow \pi^{+} \gamma\right)$ of $83 \pm 10 \mathrm{KeV}$ for the unbroken symmetry case and $73 \pm 8 \mathrm{KeV}$ for the broken symmetry case.

The first measurement of the radiative width of the $\rho$ meson(using the primakoff effect and a negative pion beam) found a value for $\Gamma\left(\rho^{-}+\pi^{-} Y\right)$ of $35 \pm 10 \mathrm{kev.} \mathrm{[15]} \mathrm{This} \mathrm{is} \mathrm{about}$ a factor of $2-2.5$ less than that predicted from the $\Gamma\left(\rho^{-} \rightarrow \pi^{-} \gamma\right) / \Gamma\left(\omega^{\circ} \rightarrow \pi^{\circ} \gamma\right)$ ratio. A reanalysis of the experiment has suggested that the presence of $A_{2}$ exchange in the strong production mechanism could substantially alter the value of the extracted radiative width. [16] A remeasurement in ig78 (Experiment E272) obtained the value $\Gamma\left(0^{-}+\pi^{-} \gamma\right)=71 \pm 7 \mathrm{KeV}$, much closer to that predicted from the above arguments. [17]

Considering the relativistic nature of the quarks in the light mesons, it seems remarkable that a non-relativistic quark model treatment provides as good agreement as it does. It has been mentioned that in the bag model treatment of mesons, the boundary conditions force the "large" component of the wave function to dominate over the "small" component throughout the interior of the bag. [18] This is just the 
approximation used in Bjorken and Drell to take the non-relativistic limit of a relativistic theory. [19] The success of the non-relativistic quark model treatment may be due to this.

B. $\rho^{+}$Production

The rate for the transition $\omega^{\circ} \rightarrow \pi^{\circ} \gamma$ is high enough, and the background low enough for a direct measurement of $\Gamma\left(\omega^{\circ}+\pi^{\circ} \gamma\right)$ to be feasible. The width for the transition $\rho^{+} \rightarrow \pi^{+} \pi^{\circ}$ is known to be approximately $150 \mathrm{MeV}$. If we estimate $\Gamma\left(\rho^{+} \rightarrow \pi^{+} \gamma\right)$ to be $100 \mathrm{KeV}$ (on the high side), we obtain $\Gamma\left(p^{+} \rightarrow \pi^{+} \gamma\right) / \Gamma\left(p^{+} \rightarrow \pi^{+} \pi^{\circ}\right)=.00067$. Background from the copious $\rho^{+}+\pi^{+} \pi^{\circ}$ decay mode (where one of the photons from the $\pi^{\circ}$ can be lost) makes a direct measurement of the $\rho^{+}+\pi^{+} \gamma$ decay very difficult. A direct search for $\rho^{-} \rightarrow \pi^{-} \gamma$ decays was reported, and after background subtraction no evidence was found for radiative decays. [20] An upper limit was quoted for $\Gamma\left(0^{-}+\pi^{-} \gamma\right) / \Gamma\left(0^{-}+\pi^{-} \pi^{\circ}\right)$ of 0.58 , corresponding to to $\Gamma\left(\rho^{-}+\pi^{-} \gamma\right)<$ 600 kev. The $\gamma \pi \rho$ coupling could also be measured in principle in the reaction $\gamma+p \rightarrow p^{\circ}+p$. [21] However, in the analysis, the one-pion exchange contribution has to be distinguished from competing mechanisms. The situation is very model dependent and unclear. 
Primakoff suggested that the $\pi^{\circ}$ lifetime could be measured in coherent $\pi^{\circ}$ photoproduction experiments, in which the nuclear coulomb field is used as a target. [22] since that time the primakoff mechanism has been used in several experiments to measure $\pi^{\circ}$ and $\eta^{\circ}$ lifetimes. [23] This mechanism also has application to the measurement of radiative widths of the form of Reaction (1.2)

$$
V \rightarrow P+\gamma
$$

by using the inverse reaction

$$
P+\gamma \rightarrow V
$$

in which the pseudoscalar meson couples to a virtual photon from the nuclear coulomb field. The reaction $\pi^{+}+\gamma+\rho^{+}+\pi^{+} \pi^{\circ}$ is shown schematically in Figure 3a. Coherent production can also proceed through hadronic exchange, as shown in Figure 3b, so a clean extraction of the radiative width involves separating the primakoff(or coulomb) and hadronic contributions. In the rest of this chapter, the characteristics and relative rates for coherent coulomb and strong production will be derived. 
Coherent processes are those in which the final nuclear state coincides with the initial nuclear ground state. [24] The production amplitude on the nucleus is a superposition of the production amplitudes of each individual nucleon.

$$
\text { Consider the reaction }
$$

$$
a+A \rightarrow a^{*}+A^{\prime}
$$

where $a$ is the incident particle, $a^{*}$ the produced particle and $A$ and $A^{\prime}$ the initial and final states of the nucleus, respectively. The kinematics of Reaction (1.39) are illustrated in Figure $4 a$.

The process can be described with the usual Mandelstam variables.

$$
\begin{aligned}
& s=(\text { center of mass energy })^{2}=\left(p_{a}+p_{A}\right)^{2}=\left(p_{a^{*}}+p_{A}\right)^{2} \\
& \begin{array}{l}
\text { (1.40a) } \\
\text { (1.40) }
\end{array} \\
& \left.q^{2}=\text { (momentum transfer }\right)^{2}=\left(p_{A}-p_{A^{\prime}}\right)^{2}=\left(p_{a}-p_{a^{*}}\right)^{2} \\
& |q|^{2}=q_{z}^{2}+q_{t}^{2} \\
& t=-q^{2}
\end{aligned}
$$

$q_{z}$ and $q_{t}$ are the components of the 3-vector momentum 
transfer parallel to and perpendicular to the original beam direction. (Note that $t$ intrinsically is a negative number. However, for the purposes of discussion, it is more convenient to consider it as being positive.) For high $s$ and low $t$, the regime being considered here, $t$ channel exchanges predominate. [25]

The minimum momentum transfer possible, $t_{\text {min, }}$ can be written

$$
\begin{aligned}
& t_{m i n}=-q_{m i n}{ }^{2}=-\left(E_{a} *-E_{a}\right)^{2}+\left(\left|\vec{p}_{a} *\right|-\left|\vec{p}_{a}\right|\right)^{2} \\
& \simeq \frac{\left(m_{a}{ }^{2}-m_{a}^{2}\right)^{2}}{4\left|\vec{p}_{a}\right|^{2}}
\end{aligned}
$$

The longitudinal momentum transfer, $q_{z}$, is small.

$$
q_{2}=q_{\mathrm{min}}
$$

Thus, we can write

$$
\begin{aligned}
t^{\prime} & =\left|t-t_{\min }\right| \\
& \simeq q_{t}{ }^{2}
\end{aligned}
$$

By the uncertainty principle, we expect typical momentum transfers for coherent interactions to assume values 
(a)
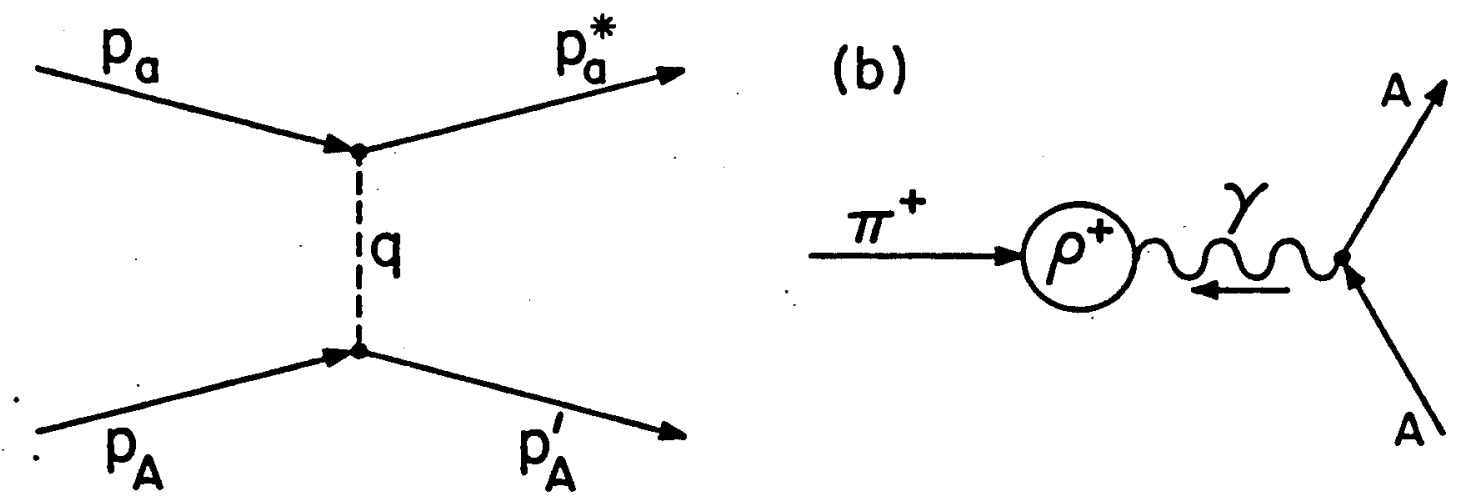

(c) GOTTFRIED - JACKSON FRAME

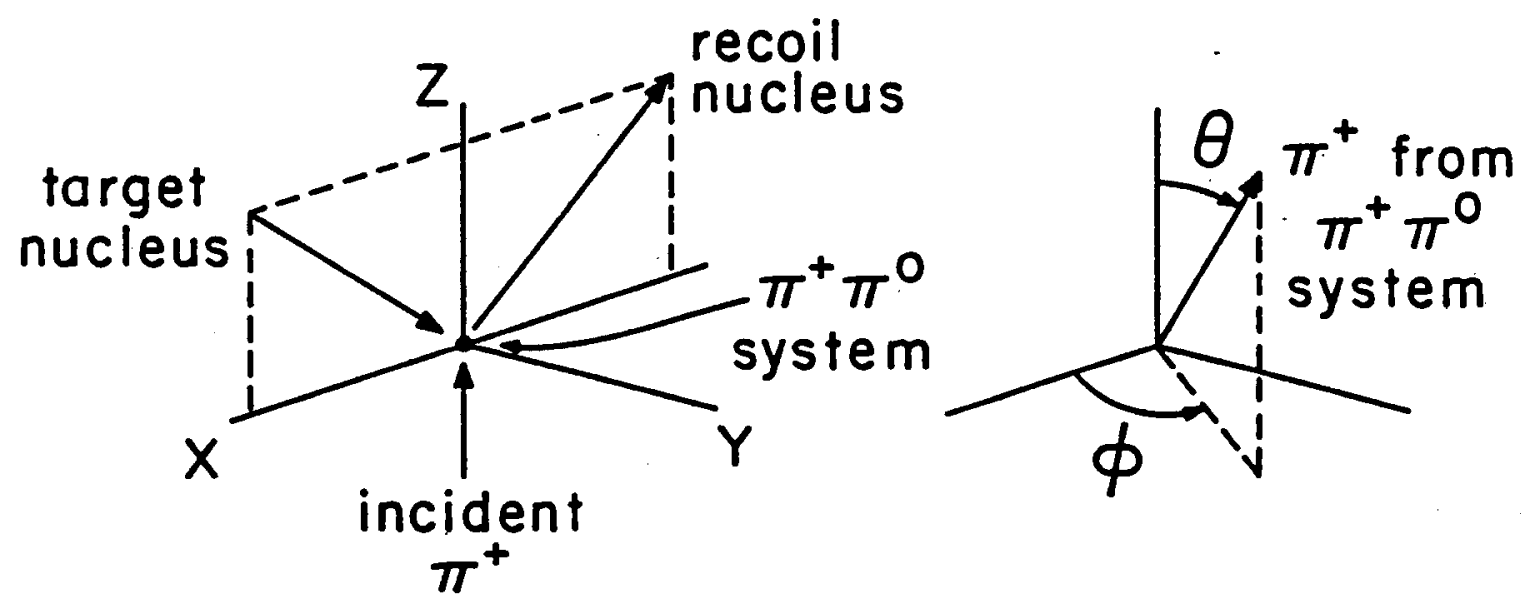

(d) HELICITY FRAME

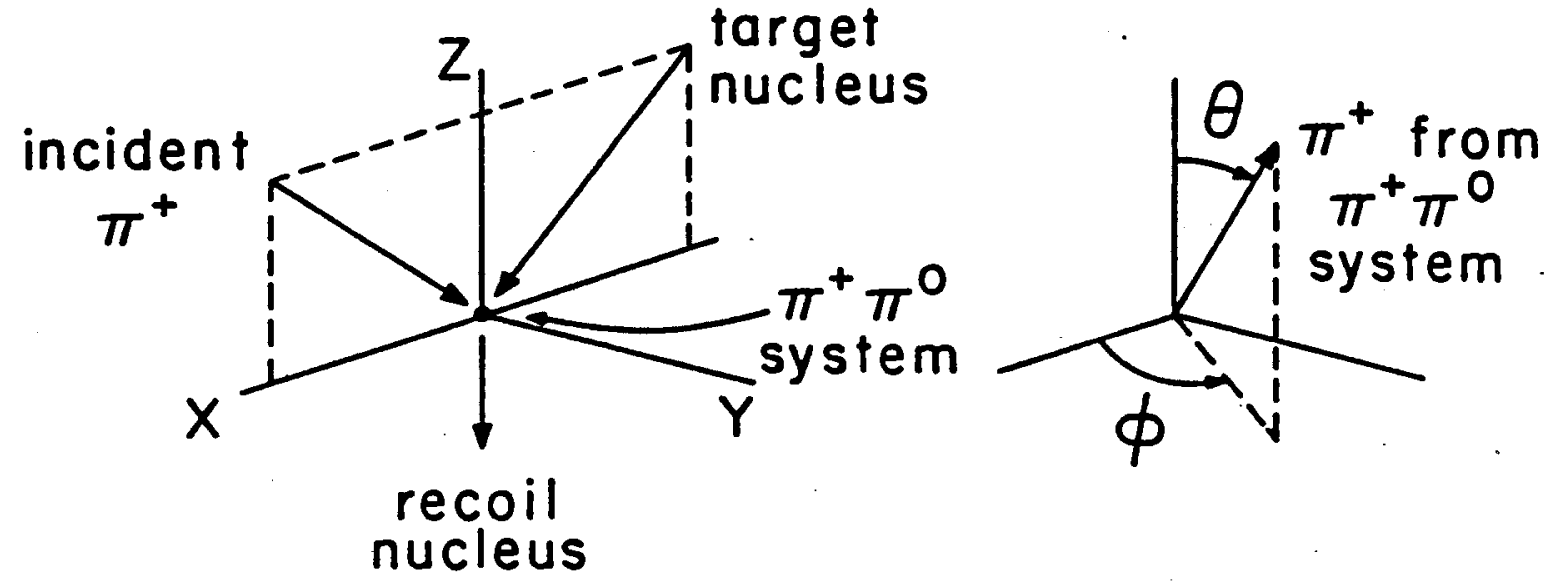


corresponding to dimensions of the nuclear radius(1.1 $\mathrm{A}^{1 / 3}$ fermi) or greater. Thus

$$
q \simeq q_{t} \leqq \frac{h c}{1.1 A^{1 / 3}}=\frac{0.2}{A^{1 / 3}}(\mathrm{GeV})
$$

For a lead nucleus, $q \leqq 35 \mathrm{MeV} / \mathrm{c}$. The kinetic energy of the recoiling nucleus, given by $q_{0}=q^{2} / 2 m_{A}$, then is $3 \mathrm{MeV}$.

Nuclear targets have an advantage over hydrogen targets in that many exchange mechanisms are enhanced or suppressed on nuclei, if the nucleus remains in its ground state. [26] For example, any amplitude proportional to the net spin of the nucleus is relatively small, since for large nuclei $J<<A$; likewise any contributions proportional to net isospin are reduced since roughly the same numbers of protons and neutrons are present in the nucleus.

For meson induced coherent reactions on a spin zero nucleus, certain selection rules can be derived as a consequence of parity and angular momentum conservation. For production in the forward direction, only changes in parity between $a$ and $a^{*}$ (in Equation (1.39)) of the form $(-1)^{\ell}$ are allowed. These processes involve production of the "unnatural parity" states of spin-parity $\mathrm{J}^{\mathrm{P}=0^{-}, 1^{+}, 2^{-}, 3^{+}, \ldots}$ Away from the forward direction, parity changes of the form 
$(-1)^{\ell+1}$ are also allowed. Exchanges of this sort involve helicity flip with the exchanged obects having $\mathrm{J}^{\mathrm{P}}=1^{-}, 2^{+}, 3^{-} \ldots$ Coherent $\rho^{+}$meson production involves exchanges of this type.

\section{G-parity conservation limits the allowed strong} exchange mechanisms for coherent dipion final states produced by incident pions. The selection rule can be written $G_{a} G_{a}{ }^{*}$ exchange ${ }^{=+1}$. One immediate consequence of this rule is that dipion final states cannot be produced diffractively (by the exchange of the vacuum quantum numbers or pomeron exchange). In Coulomb production of dipion states, G-parity conservation allows only the isoscalar part of the photon to couple to the $\gamma \pi \rho$ vertex(the isoscalar part of the photon has $G=-1$, the isovector part of the photon has $G=+1$ ).

Strong processes with $I=1$ exchanges are possible, but as mentioned previously, are suppressed in nuclei due to partial cancellation of contributions from protons(isospin up) and neutrons (isospin down). It is thus expected that $w$ exchange $\left(J^{P}=1^{-}, I^{G}=0^{-}\right)$ will dominate

over

$\mathrm{A}_{2}$ exchange $\left(\mathrm{J}^{\mathrm{P}}=2^{+}, \mathrm{I}^{\mathrm{G}}=\mathrm{I}^{-}\right)$in hadronic production of the $\rho^{+}$.

The generalized pauli principle requires that the isotopic spin of the dipion final state be even for even relative angular momentum and odd for odd relative angular momentum. From this and the above discussion it can be 
concluded that coherently produced dipion states will predominately be found in states with $I=1$ and $\mathrm{J}^{\mathrm{P}}=1^{-}, 3^{-}, 5^{-} \ldots$

The decay characteristics of coherently produced $\rho^{+}$ mesons can be determined from the properties of the possible exchange particles, $\gamma$ and $\omega^{\circ}$. In Figure $4 b$, Reaction (1.1) is depicted in the rest frame of the $\rho^{+}$meson. If we regard the incident pion as a plane wave, we can expand it in terms of the spherical harmonic wave functions. The photon has two possible helicity states, \pm 1 . The spin of the produced $\rho^{+}$ must then either point along or opposite the direction of the incident pion. A similar argument can be made for $\omega^{\circ}$ exchange(but in this case three helicity states are possible though the state with zero projection cannot couple to a plane wave to form a $\mathrm{p}^{+}$).

A reference frame useful in describing coherent production is the Gottfried-Jackson frame, the definition of which is provided in Figure 4c. [27] The charged pion from the $\rho^{+}+\pi^{+} \pi^{\circ}$. decay should exhibit an angular distribution characterized by $\left|Y_{I}^{I}(\theta, \phi)+Y_{1}^{-1}(\theta, \phi)\right|^{2}$ or $\sin ^{2} \theta \sin ^{2} \phi$. In Figure $4 d$ the $s$ channel helicity frame is defined. For Coulomb production at high energy, the two frames are practically identical.

\section{B. 2 Coulomb Production}


The cross section for the coulomb production of a state $a^{*}$ with mass $m$ in the reaction $a+A \rightarrow a^{*}+A^{\prime}$ can be written (in the high energy limit) as [28]

$$
\frac{d \sigma}{d t d m^{2}}=\frac{\dot{z}^{2} \alpha}{\pi} \frac{\sigma_{\gamma}\left(m^{2}\right)}{\left(m^{2}-m_{a}^{2}\right)} \frac{t-t_{m i n}}{t^{2}}\left|F_{e m}(t)\right|^{2}
$$

$z$ is the nuclear charge, $\alpha$ the fine structure constant, $m_{a}$ the mass of the incident particle and $F_{\text {em }}(t)$ the electromagnetic form factor. Fem (t) has been modified to take into account the nuclear absorption of the incoming and outgoing states. (This will be discussed in more detail in Chapter VI.) $\sigma_{\gamma}\left(m^{2}\right)$ is the total cross section for the reaction $a+\gamma \rightarrow a^{*}$ at a $\gamma$-a center of mass energy $E_{\mathrm{cm}}=\mathrm{m}$. If the final state $a^{*}$ is a single particle of mass $m, \sigma_{\gamma}\left(m^{2}\right)$ can be written

$$
\sigma_{\gamma}\left(m^{2}\right)=\frac{2 \pi}{k^{2}}\left(\frac{2 J_{a}^{*}+1}{2 J_{a}+1}\right) \frac{\Gamma\left(a^{*}+a+\gamma\right) \Gamma\left(a^{*}+x\right)}{\left(m-m_{0}\right)^{2}+(\Gamma / 2)^{2}}
$$

$J_{a}$ is the spin of the incident particle, $J_{a}$ * the spin of the outgoing particle, $m_{0}$ is the resonance mass of $a^{*}$, and $k$ is the momentum of the photon in the center of mass system of $a^{*}$. $k$,again, can be written

$$
k=\frac{m^{2}-m_{a}^{2}}{2 m}
$$


$\Gamma\left(a^{*}+a \gamma\right)$ is the mass dependent partial width(See Chapter VI) for the reaction $a^{*}+a+\gamma$ and $\Gamma\left(a^{*}+x\right)$ is the mass dependent partial width for the reaction $a^{\star}+x$ where $x$ is the final state of $a^{*}$ that is measured experimentally. (For $a^{*}=\rho^{+}, x=\pi^{+} \pi^{\circ}$. , The factor of $\Gamma$ in the denominator refers to the total width of $a^{*}$. Substituting Equation (1.46) into Equation (1.45), we obtain

$$
\begin{aligned}
\frac{d \sigma}{d t d m^{2}}= & \left.4 \pi \alpha z^{2} \frac{2 J_{a}^{*+1}}{2 J_{a}+1}\right) \frac{m^{2}}{\left(m^{2}-m_{a}{ }^{2}\right)^{3}} \frac{\Gamma\left(a^{*}+a \gamma\right) \Gamma\left(a^{*}+x\right)}{\Gamma} \\
& \frac{1}{\pi} \frac{\Gamma / 2}{\left(m-m_{0}\right)^{2}+(\Gamma / 2)^{2}} \frac{t-t_{m i n}}{t^{2}}\left|F_{e m}(t)\right|^{2}
\end{aligned}
$$

Integrating over $\mathrm{m}^{2}$

$$
\begin{aligned}
\frac{d \sigma}{d t}=4 \pi \alpha z^{2} & \left.\frac{2 J_{a}^{*+1}}{2 J_{a}+1}\right) \int \frac{m^{2}}{\left(m^{2}-m_{a}{ }^{2}\right)^{3}} \frac{\Gamma\left(a^{*}+a \gamma\right) \Gamma\left(a^{*}+x\right)}{\Gamma} \\
& \frac{1}{\pi} \frac{\Gamma / 2}{\left(m-m_{0}\right)^{2}+(\Gamma / 2)^{2}} \frac{t-t_{m i n}}{t^{2}}\left|F_{e m}(t)\right|^{2} \mathrm{dm}^{2}
\end{aligned}
$$

If we make the nar row resonance assumption, the features of the above formula become more transparent. For $\Gamma<m_{0}$

$$
\frac{1}{\pi} \frac{\Gamma / 2}{\left(m-m_{0}\right)^{2}+(\Gamma / 2)^{2}}=\delta\left(m-m_{0}\right)
$$


If $\Gamma\left(a^{*}+x\right) \simeq \Gamma$ (true for the $\rho^{+}$case) Equation (1.49) becomes

$$
\frac{d \sigma}{d t}=8 \pi \alpha z^{2}\left(\frac{2 J_{a}+1}{2 J_{a}+1}\right) \Gamma_{o}\left(a^{\star}+a \gamma\right)\left(\frac{m_{o}}{m_{o}^{2}-m_{a}^{2}}\right)^{3} \frac{t-t_{\min }}{t^{2}}\left|F_{e m}(t)\right|^{2}
$$

where the radiative width $\Gamma_{0}$ is evaluated at the resonance mass $m_{0}$. (The above approximation will be discussed in more detail in Chapter VI).

Some important features of Coulomb production can be deduced from Equation 1.51:

1. The diffential cross section is sharply peaked at low $t$ due to the presence of the photon propagator $1 / t^{2}$. The cross section vanishes at $t=t_{\min }(a$ consequence of current conservation for the electromagnetic field [29]), rises rapidly with to peak at $t=2 t_{\mathrm{min}}$, and then falls roughly as $1 / t$. The peak position is almost independent of target nucleus, i.e. , the form factor varies much more slowly with $t$ than the photon propagator term does.

2. From Equation (1.4Ib) it is seen that $t_{m i n}$ approaches 0 as $I /\left|\nabla_{a}\right|^{2}$. For an incident pion 
momentum of $200 \mathrm{GeV}, t_{m i n}$ for $\rho^{+}$production (at 770 $\mathrm{MeV}$ ) is approximately $2 \times 10^{-6} \mathrm{GeV}^{2}$.

3. The height of the peak is proportional to $\mathrm{z}^{2}\left|\mathrm{D}_{\mathrm{a}}\right|^{2}$.

4. As $\left|\vec{p}_{a}\right|$ approaches infinity, the integrated cross section increases as $\mathrm{z}^{2} \log \left|\mathrm{t}_{\mathrm{a}}\right|$. This is due to the decrease of $t_{\text {min }}$ with increasing momentum.

5. For high incident momentum most of the coherent Coulomb production takes place at values of $q(=V|t|)$ corresponding to distances larger than the nuclear radius. For $q=\sqrt{2} q_{m i n}$ we can write

$$
b=\frac{h c}{\sqrt{2} q_{m i n}}=\frac{.28 p}{m_{a}{ }^{*}-m_{a}^{2}}
$$

where $b$ is the impact parameter. For $\rho^{+}$production at the resonance mass at an incident momentum of 200 Gev, $b=56$ fermi. The radius of a lead nucleus is approximately 7 fermi $\left(1.1 \mathrm{~A}^{1 / 3}\right)$. Thus the behavior of the differential cross section will not be particularly sensitive to the parameters chosen to describe the form factor. 
As mentioned previously, coherent production can also proceed by hadronic exchange. For $\rho^{+}$production from incident pions, $\omega^{\circ}$ exchange predominates. The differential cross section can be parameterized in the form [30]

$$
\frac{d \sigma}{d t}=A^{2} C_{s}\left|t-t_{\min }\right|\left|F_{h}(t)\right|^{2}
$$

where $A$ is the atomic weight, $C_{S}$ is a parameter describing the strength of the process on a single nucleon, and $F_{h}(t)$ is the hadronic form factor modified to include initial and final state absorption. Some of the germane characteristics of the above cross section are listed below:

1. The behavior of the factor $C_{s}$ can be determined from Regge theory. $C_{s}$, for $\omega^{\circ}$ exchange, should fall linearly with the incident momentum, that is, $C_{s} \alpha$ $1 /\left|\vec{p}_{\pi}+\right|$. Thus the integrated hadronic cross section roughly falls as $1 /\left|\vec{p}_{\pi}+\right|$. The integrated cross section for coulomb production was found to increase as $\log \left|t_{\pi}+\right|$. Thus at high enough energies coulomb production will predominate over strong production.

2. In the forward direction the square of the hadronic form factor varies roughly as $e^{-b t}$ where $b=R^{2} / 4, R$ being the nuclear radius. Substituting this form of. 
$\left|F_{h}(t)\right|^{2}$ into Equation (1.53) enables us to determine the behavior with $t$ of the differential cross section. The cross section vanishes in the forward direction $\left(t=t_{\min }\right)$, rises to a peak at $t \simeq 4 / R^{2}$ and then falls due to the depressing effect of the form factor.

3. The shape of the strong cross section is roughly independent of energy. Since the Coulomb cross section peaks at $t=2 t_{\min }$ and $t_{\min } \alpha 1 /\left|\vec{p}_{\pi}+\right|^{2}$, the separation in $t$ of the coulomb and strong contributions will be greater at higher energies. For a lead target at $200 \mathrm{GeV} / \mathrm{c}$, the Coulomb term peaks at $t=4 \times 10^{-6} \mathrm{GeV}^{2}$ and the strong term at $t=.003$ $\mathrm{GeV}^{2}$. The separation is better for lower $\mathrm{A}$ since the peak of the strong cross section falls with the square of the nuclear radius.

4. The strong cross section is more sensitive to the parameters used to describe the nucleus than the Coulomb cross section. The hadronic form factor, $F_{h}(t)$, was calculated with an optical nodel (see Chapter VI) in which the nuclear density is taken to be the same as the charge density. In the absence of absorption the effective A-dependence of the integrated cross section becomes $A^{2 / 3}$. Absorption 
effects reauce the strong cross section even further. Since Coulomb production increases as $z^{2}$, the ratio of coulomb to strong contributions will increase with heavier nuclei.

The Coulomb and strong amplitudes can interfere. The total cross section must then be written in the form

$$
\frac{d \sigma}{d t}=\left|T_{c}+e^{i \phi T_{s}}\right|^{2}
$$

where $\operatorname{d\sigma /dt(Coulomb)}=\left|T_{c}\right|^{2}$ and $\operatorname{d\sigma /dt(strong)}=\left|T_{s}\right|^{2}$ and $\phi$ is the relative phase between the two amplitudes. This equation, smeared by the observed resolution, was used for the experimental fits to the observed $\rho^{+} t$ distributions. There were three parameters in the fit: the $\rho^{+}$radiative width $\Gamma_{0}\left(\rho^{+}+\pi^{+} \gamma\right)$, the parameter $C_{s}$ for $\omega^{\circ}$ exchange, and the relative phase $\phi$. The fitting process will be described in more detail in Chapter VI. 
A. General Considerations

Characteristics of the reaction we wished to measure

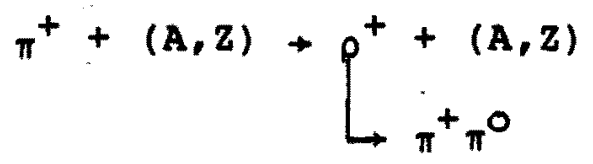

placed strong constraints on the design of the apparatus for this experiment. The size of the total cross section for this reaction can be estimated by integrating the theoretical differential cross section Equation (1.54), over t. At 200 GeV incident momentum, using a value for the radiative width of $70 \mathrm{KeV}$, one obtains about 1.0 millibarn(mb). The total $\pi^{+} \mathrm{Pb}$ cross-section at $200 \mathrm{GeV}$ is about $2000 \mathrm{mb}$. Thus the reaction of interest corresponds to about 0.058 of the total cross-section. Therefore, one very important consideration in the design of the experiment was that a trigger be devised to select only the small fraction of the total interactions that were of interest. 
An unambiguous extraction of the radiative width requires an adequate separation of the two coherent contributions, Coulomb and strong. As discussed in Chapter I, the two processes dominate in different t regions; the Coulomb process at very small t and the strong process at moderate $t$. The experimental resolution smears out the theoretical $t$ distribution and thereby produces an ambiguity in the size of the two contributions. The experiment was designed to have good resolution in $t$ so as to permit a clean extraction of the radiative width. The desire for optimal $t$ resolution led. to two constraints: because multiple Coulomb scattering in the target leads to a degradation of resolution, only thin targets could be used, and, in order to determine the $t$ of the reaction, high precision detectors were needed to measure the positions and energies of the decay products. The first constraint necessitated that the targets were kept to a few tenths of a radiation length; the second constraint meant that a magnetic spectrometer, equipped with drift wire chambers and a fine-grained liquid argon calorimeter, was necessary for adequate measurement. In the following sections the design and operation of the E272 spectrometer will be discussed in more detail.

B. Beam 
The experiment was conducted in the M1-East beamline at Fermilab. The Ml-East beam originated in interactions of $400 \mathrm{GeV}$ primary protons with a $38 \mathrm{~cm}(1.25$ interaction lengths) beryllium target. A secondary positive beam of 200 GeV momentum was transported down to our experiment. The momentum spread of the beam was \pm 18 and the maximum acceptance for the tune which we used was approximately 2 microsteradians. During our experiment the production angle for the MI beam varied from 1.9 milliradians to 3.6 milliradians, with most of the data taken at angles less than 2.5 milliradians.

The Ml beam was a standard 3-stage beam [31]. The first stage provided dispersion for momentum selection. The second stage recombined the momentum, while the third stage provided a $50 \mathrm{~m}$ long parallel section which could be used for identifying particles using differential Cerenkov counters. A schematic of the beamline, along with ray traces of selected beam particles, is shown in Figure 5 .

At its nominal production angle of $3.6 \mathrm{mrad}$ and at a momentum of $+200 \mathrm{GeV}$, the Ml beam was composed of $2.58 \mathrm{~K}^{+}$, $14.08 \pi^{+}$, and 83.58 p. (These percentages refer to measurements $400 \mathrm{~m}$ downstream of the production target. [32]) In this experiment, however, the MI beamline was run under somewhat different conditions then in previous 


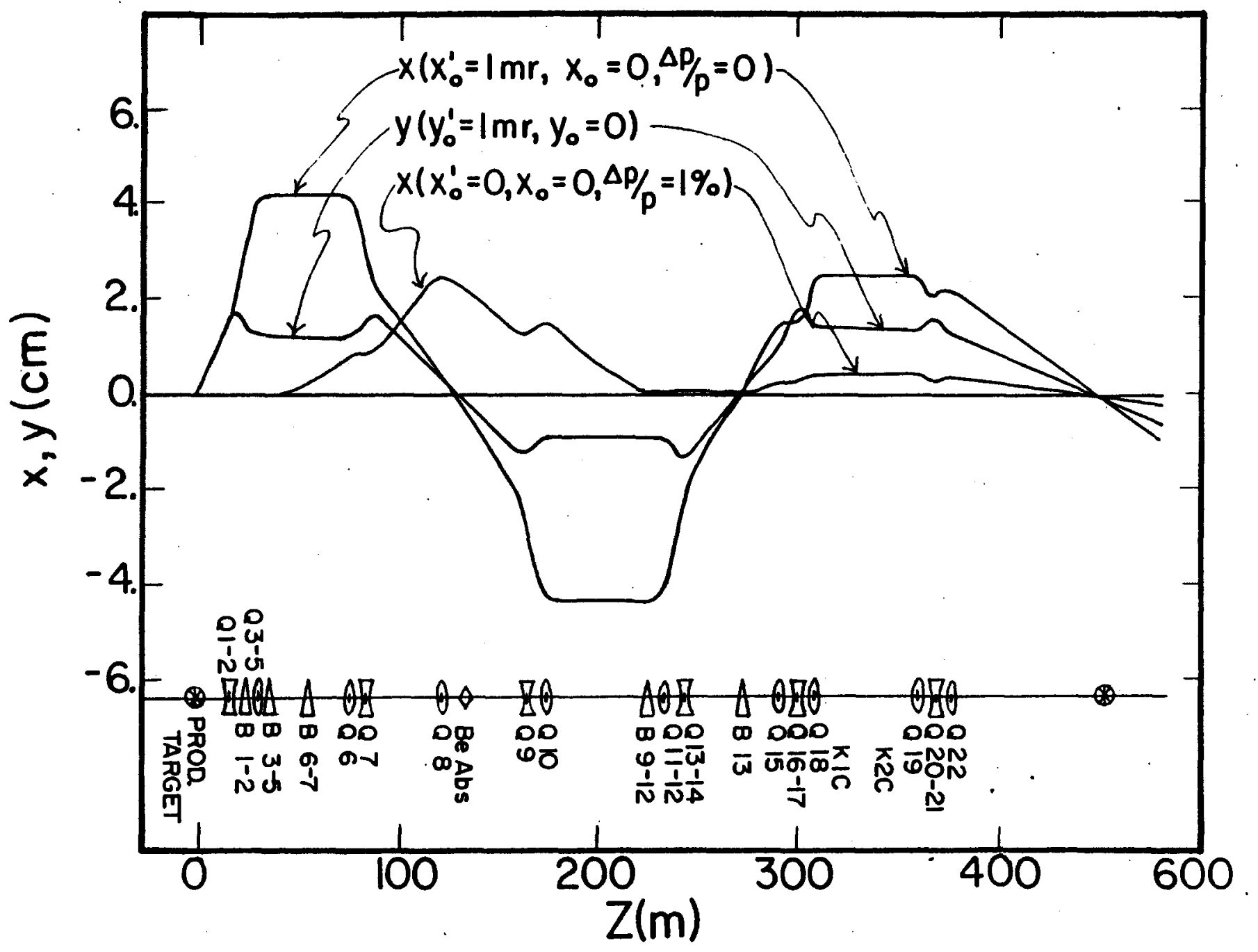

Figure 5 
experiments. A 1978 run of E272 used a $156 \mathrm{GeV}$ negative beam to extract a precision measurement of the radiative width $\Gamma\left(\rho^{-}+\pi^{-} \gamma\right)$. The radiative width $\Gamma\left(K^{*-}+k^{-} \gamma\right)$ was also measured during this run. However, due to the low kaon flux in the beam, a precision measurement was not possible. Because of the nature of the spectrometer it was undesireable to run at intensities greater than $10^{6}$ particles/second. To increase the number of kaons in the beam and yet keep the intensity reasonably low, it was decided to place a beryllium absorber at the first focus in the MI beamiine. The proton absorption cross section on beryllium is larger than either the pion or kaon absorption cross-section. The effect of the placement of the beryllium in the beamline was to produce an enhancement of the $\mathrm{K}^{+}$fraction, and to a somewhat lesser extent of the $\pi^{+}$fraction in the beam. [33] A positive beam was chosen because the ratio of the absorption cross section for the majority particle(proton) to the kaon absorption cross section is larger than the ratio of the pion(majority particle in negative beam) absorption cross section to the kaon absorption cross section, and also because the initial positive kaon flux is larger than the negative kaon flux.

The beryllium absorber consisted of 4 metal blocks, $10 \mathrm{~cm} \times 10 \mathrm{~cm}$ in cross-section, ranging in length from $15 \mathrm{~cm}$ to $125 \mathrm{~cm}$. Any combination of the four could be remotely inserted into the beam providing a maximum absorber length of 
$221 \mathrm{~cm}$. The beryllium was placed at the momentum dispersed focus to minimize the effect of multiple scattering in the absorber. For a monochromatic beam, the divergence at this focus was $\pm 0.4 \mathrm{mrad}$ in the bending plane and $\pm 0.8 \mathrm{mrad}$ in the vertical plane. Multiple scattering of the beam through $2 \mathrm{~m}$ of beryllium contributed only $0.2 \mathrm{mrad} \mathrm{rms}$ to the divergence in each plane. Nevertheless effects of the increased multiple scattering could be discerned in the degraded response of the downstream Cerenkov counters. Slight adjustments were required.in the beam optics whenever the absorber length was changed.

In Figure 6 are shown the beam fractions of pions, kaons, and protons for a $3.6 \pm 0.45 \mathrm{mrad}$ (with $\mathrm{p}_{t}=720 \pm 90 \mathrm{MeV}$ ) production angle as a function of absorber length. Figure 7 shows the beam fraction for two beryllium absorber lengths as a function of transverse momentum at the production target.

The tagging of incident beam particles was achieved with two differential Cerenkov counters, $\mathrm{K} 1$ and $\mathrm{R} 2$, and one threshold Cerenkov counter, R3. (See Figure 5) Counters R.l and R2 were located in the "parallel" section of the beam which consisted of the region between $305 \mathrm{~m}$ and $360 \mathrm{~m}$. In this region the beam had a small angular divergence. Counter R3 was located in the beam region between $378 \mathrm{~m}$ and $408 \mathrm{~m}$. Counter $\mathrm{Rl}$ was $32.3 \mathrm{~m}$ long and was sensitive to Cerenkov 


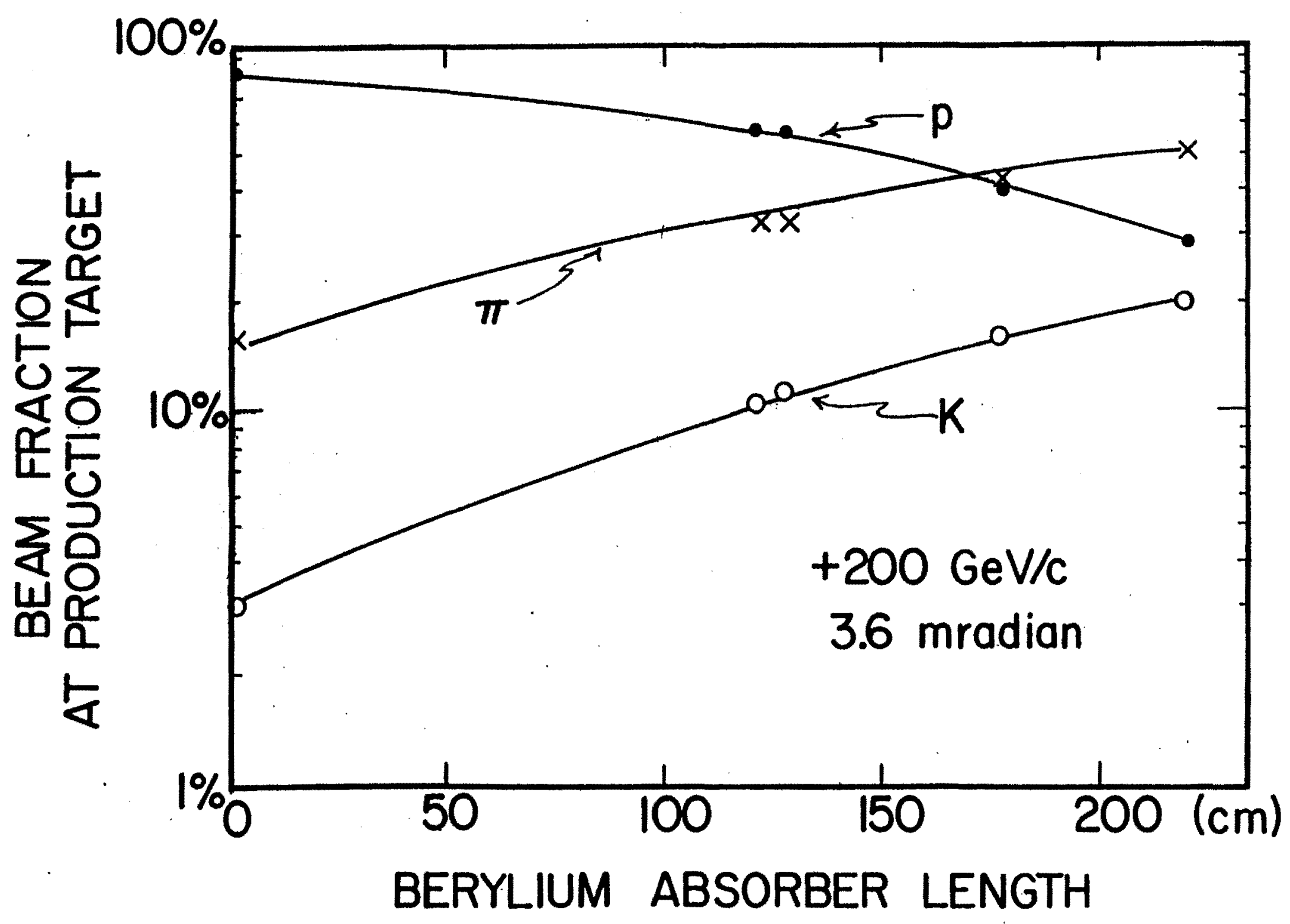




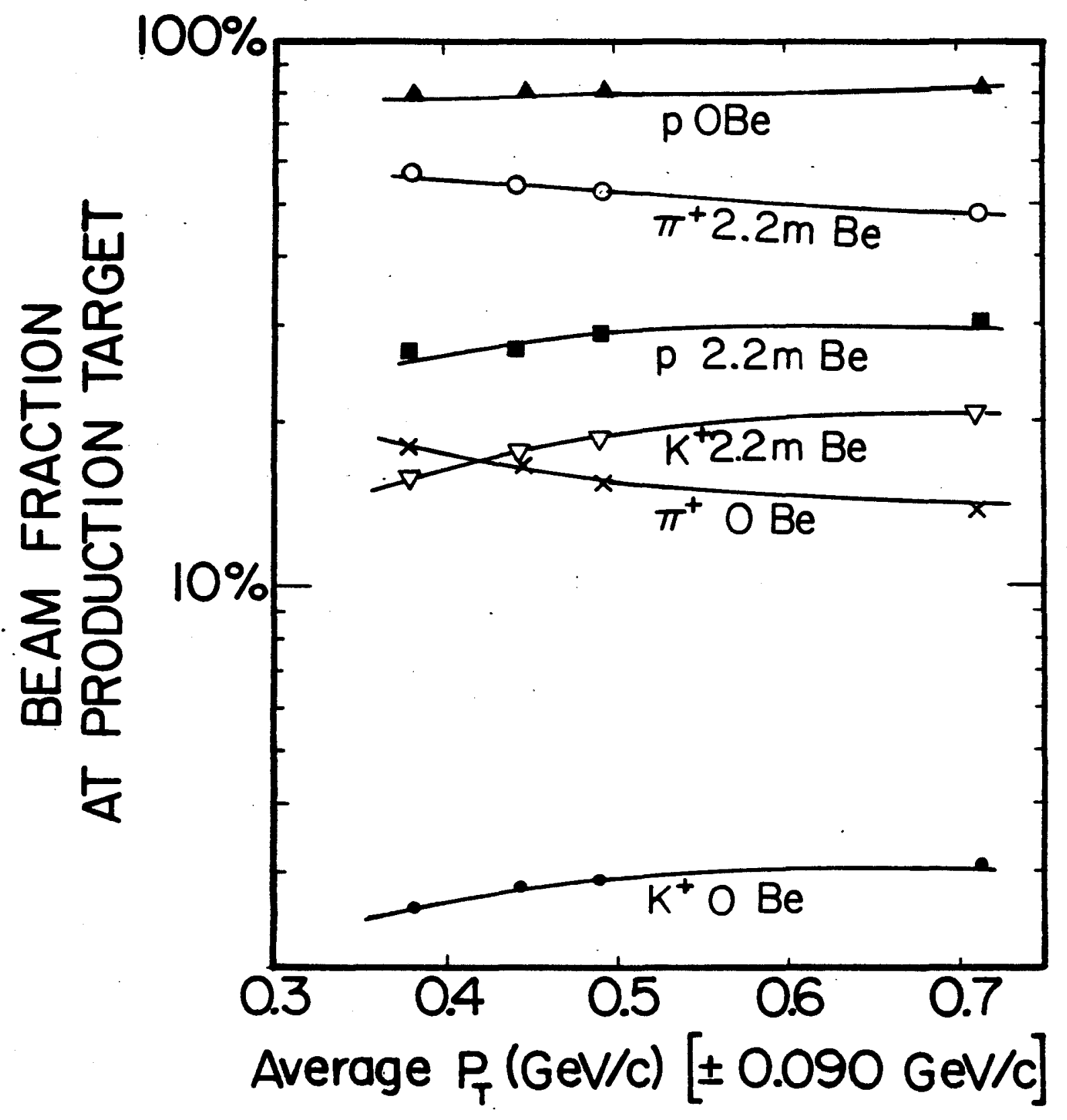

Figure 7 
radiation at an angle of $7.5 \mathrm{mrad}$. Counter $\mathrm{k} 2$ was $16.2 \mathrm{~m}$ long and was sensitive to radiation at an angle of $15 \mathrm{mrad}$. Both counters had two concentric rings of 6 phototubes to view the radiated light. The outer ring, available to be used as a veto, was not needed in this experiment. signals from adjacent phototubes of the inner ring were combined to form three logic signals. Identification of a beam particle then required either a 2 fold or a 3 fold coincidence of these logic signals.

Counter $\mathrm{R3}$ (30 $\mathrm{m}$ long) had two phototubes, one of which was sensitive to light at angles less than $7.5 \mathrm{mr}$ ad and one which was sensitive to light emitted at angles greater than $7.5 \mathrm{mrad}$. Positive identification of a beam particle required that a signal from the inner phototube not be accompanied by a signal from the outer phototube.

Helium was used as the radiating medium for all 3 Cerenkov counters. The pressures in the counters were adjusted so that $\mathrm{K} 1$ was used to identify kaons, $\mathrm{K} 2$ to identify pions and $\mathrm{K3}$ to identify protons. Pressure curves for the three counters are shown in Figures 8,9 , and 10. These curves were taken with $1.2 \mathrm{~m}$ of beryllium in the beamline. ( $B$ in the figure captions is for beam particle, the definition of which will be provided in section F). 
Figure 8 shows the pressure curve for Kl for 2-fold and 3-fold coincidences. During the run, a compromise had to be struck between maximizing the kaon flux by inserting more beryllium and between maintaining good efficiency in Cerenkov tagging.'As a result of this compromise, $\mathrm{Kl}$ was somewhat inefficient. For a 2-fold coincidence the counter was approximately $97 \%$ efficient, independent of absorber thickness. The 3-fold coincidence, however, was only $80 \%$ efficient using $2.1 \mathrm{~m}$ of beryllium compared to 908 using no absorber. Present under the kaon peak is a pion contamination of about $1.5 \%$. The $\mathrm{K} 2$ counter was $98 \%$ efficient using $2.1 \mathrm{~m}$ of beryllium. There was $a 0.5 \%$ contamination of kaons under the pion peak. The pion and kaon separations were more than adequate for this experiment. Corrections for the above mentioned contaminations were applied in the calculation of decay rates and cross sections.

The sundry beamline elements were controlled through a Fermilab computer system, a control console for which was present in the experimental trailer. The beam spot size could be monitored using several integrating wire chambers located at various spots in the beam line. The beam intensity could be controlled by using horizontal and vertical collimators present at several locations in the beamline. Primarily, it was collimator C4V,located at the first focus, that was used to alter the intensity. The beam 


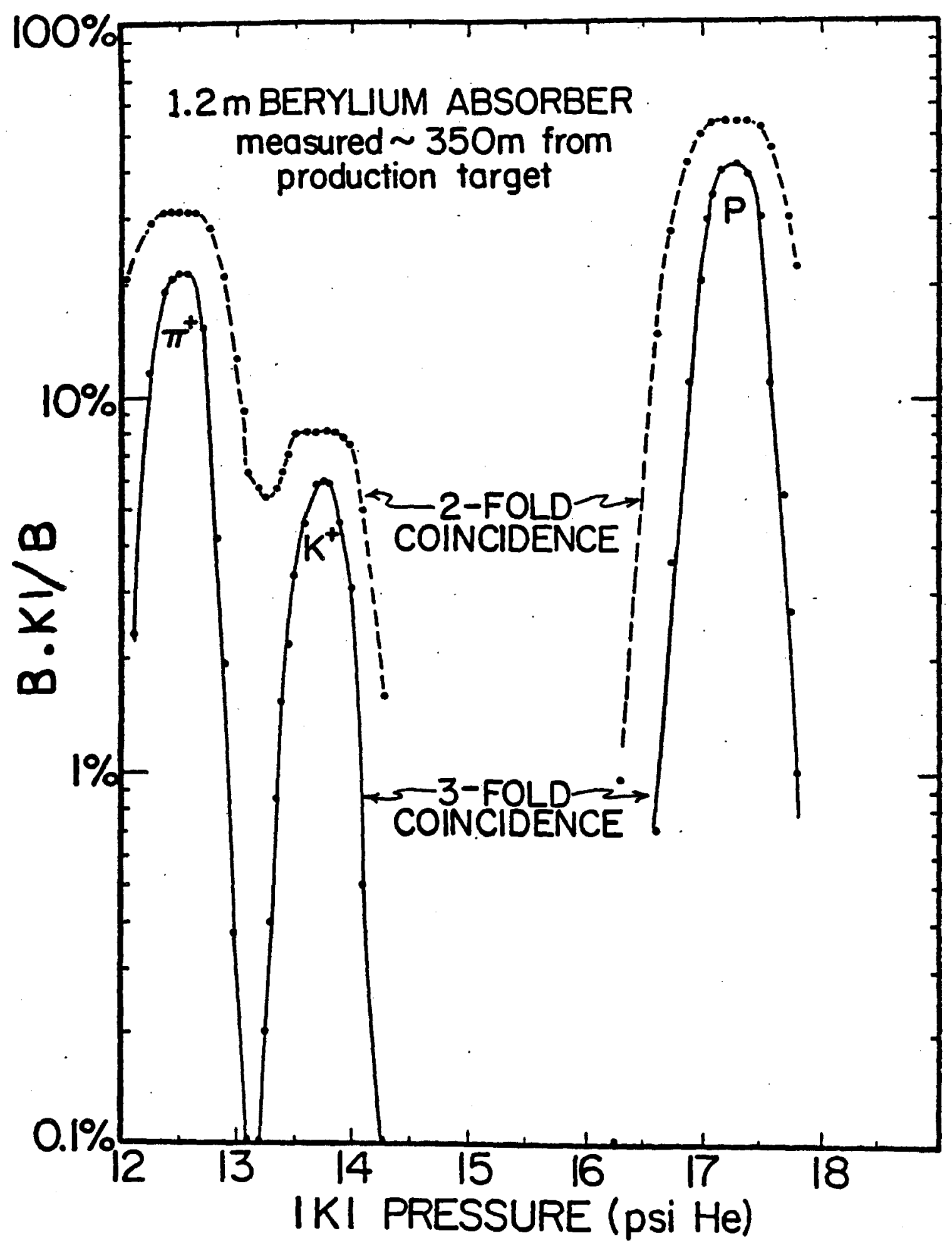

Figure 8 


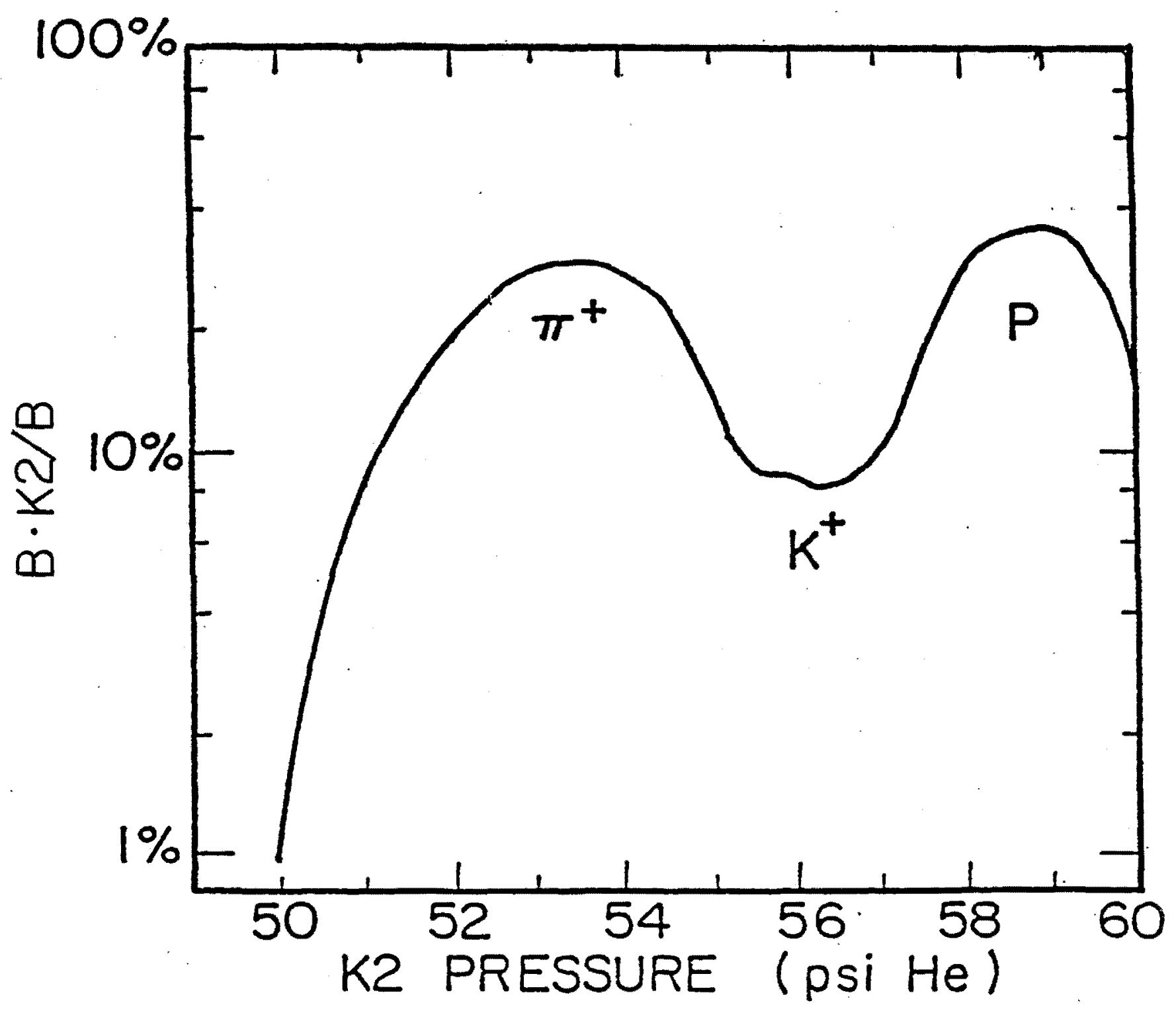

Figure 9 


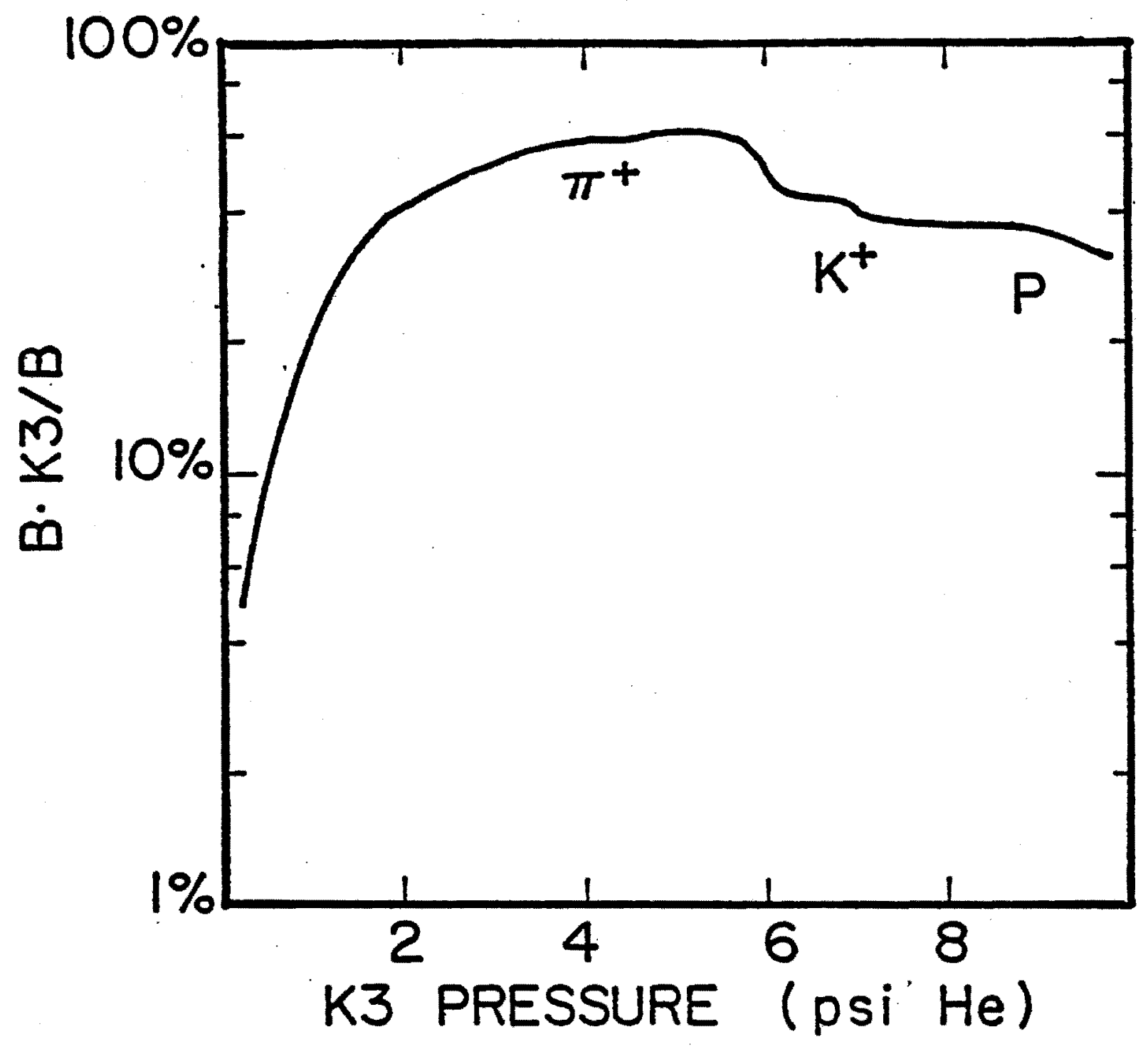

Figure 10 
flux varied in the experiment from $2-5 \times 10^{5} \mathrm{particles} / \mathrm{second}$. The main accelerator control provided a series of reference times for the experimenters to indicate the beginning and end of "flattop", the period of extraction of the primary beam. These reference times were used to gate the experimental electronics.

C. Targets

Three nuclear targets were used in this experiment: carbon, copper, and lead. The three targets were chosen to cover a range of $A$ and $Z$ so as to examine the coulomb and strong production mechanisms in regimes of different relative strength. The properties of these targets are shown in Table I. The targets were $3.75 \mathrm{~cm}$ by $3.43 \mathrm{~cm}$ in their transverse dimensions, and were glued in place in a lucite holder for ease of handling. A balance was struck in the selection of the target thickness between the desire for high data rate and the desire to keep the number of radiation lengths to a minimum. As mentioned previously, multiple scattering in the target contributes substantially to the $t$ resolution of the experiment and, in fact, was the dominant contribution in the case of the copper target. There will be more discussion of this point in Chapter VI.

D. Particle Detection 


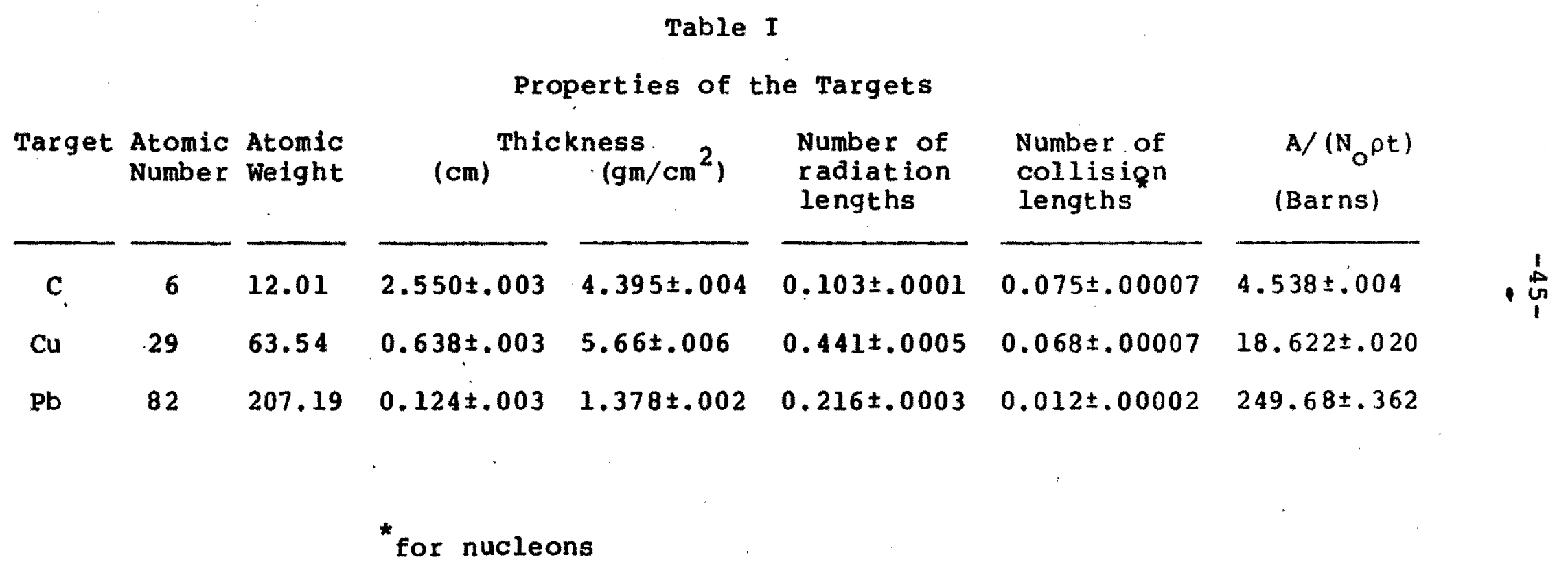


The final state of Reaction (1.1) consisted of one charged pion and two photons from the decay of the neutral pion. Particles were detected using the spectrometer shown in Figure 11. The spectrometer consisted of two sets of proportional chambers, one set of drift chambers and a liquid argon calorimeter.

\section{D.l Charged Particles}

Charged particles were detected with two sets of proportional chambers and one set of drift chambers. Immediately upstream of the target were two multiwire proportional chambers, $\mathrm{Jl}$ and $\mathrm{J} 2$, which were used to define incident beam tracks. Jl and J2 each consisted of 4 wire planes, 2 measuring $X$ position and 2 measuring $Y$ position. The wire separation for each plane was $1 \mathrm{~mm}$, but each plane in $X$ and $Y$ was staggered with respect to the other, providing in an effective wire spacing of $0.5 \mathrm{~mm}$. In this way an angular resolution of $\pm 0.015 \mathrm{mrad}$ was achieved for beam tracks. Signals from one $X$ plane in each of $J I$ and $J 2$ were used for on-line event selection in a low-level trigger processor (See Section E).

Each of the proportional chambers $\mathrm{P} 1 \mathrm{X}, \mathrm{P} 1 \mathrm{Y}$ and $\mathrm{P} 2$ also had two staggered planes. The wire spacing on each plane was $2.5 \mathrm{~mm}$, leading to an effective separation of $1.25 \mathrm{~mm}$. PlX 

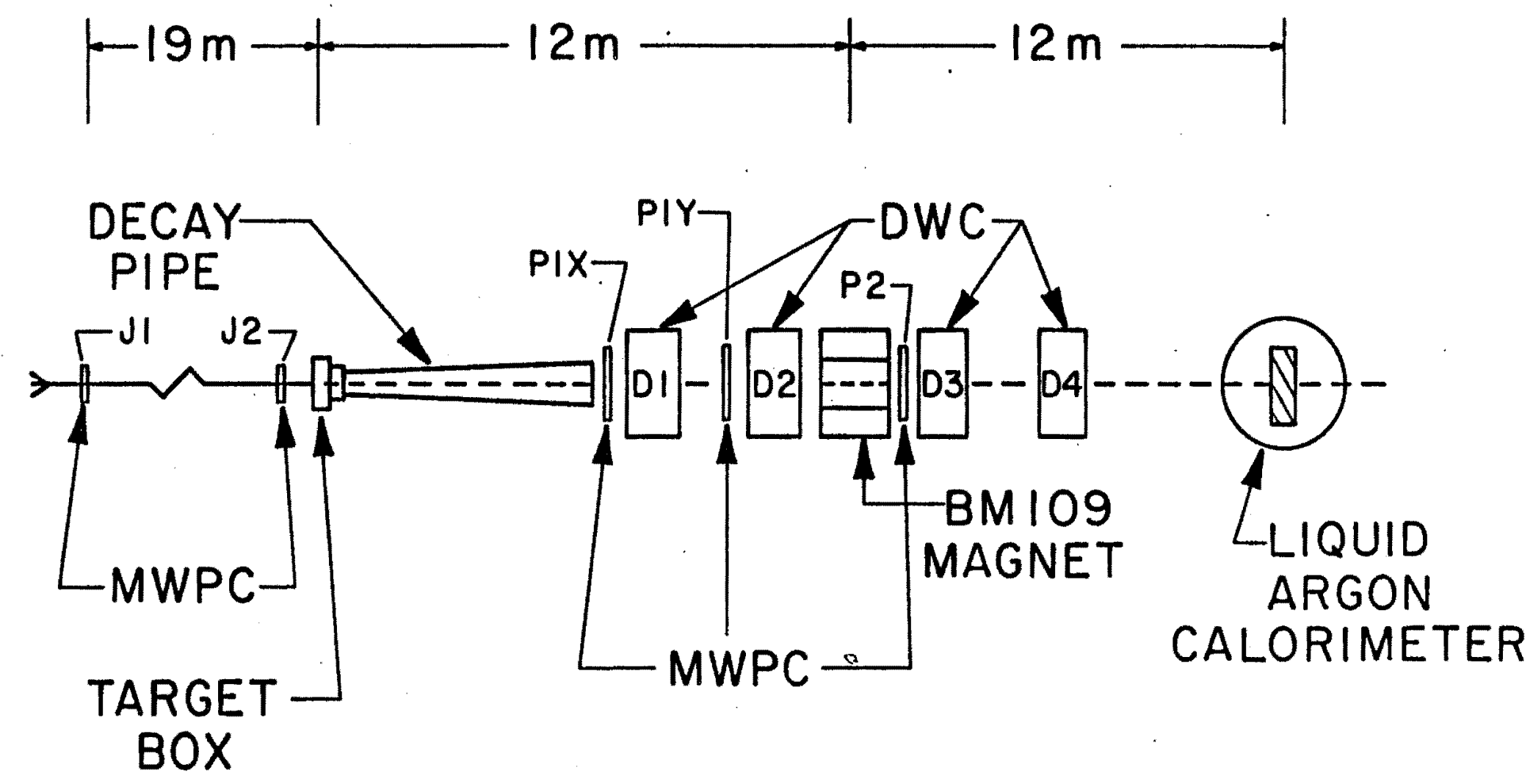
and $P 2$ measured the $X$ position of charged particles while PIY measured the $Y$ position. Signals from PIX were also used for the trigger processor mentioned above.

There were 24 drift chamber planes arranged in 4 groups, D1-D4. D1 and D2 were upstream of the magnet while D3 and D4 were downstream. D1 consisted of $2 X$ planes, 2 Y planes and 20 planes in a common gas volume. The $U$ planes were at an angle of +30 degrees with respect to the vertical. D2 was identical to $D 1$ except that the $U$ planes were replaced by $V$ planes which had wires at an angle of -30 degrees with respect to the vertical. The active areas of D1 and D2 were $20.3 \mathrm{~cm}$ vertically by $61 \mathrm{~cm}$ horizontally. The active areas matched the aperture of the BM109 magnet.

D3 and D4 consisted of 6 planes, each with its own separate gas volume. The planes in D3 had an active area of $45.7 \mathrm{~cm}$ vertically by $111.8 \mathrm{~cm}$ horizontally. Two planes measured $x$, two planes were $U(+18.5$ degrees with respect to the vertical) and two planes were $v(-18.5$ degrees with respect to the vertical). The D4 group was identical to D3 group except that the active area was $61.6 \mathrm{~cm}$ vertically by $152.4 \mathrm{~cm}$ horizontally.

The cell size in each of the 24 drift planes was 2 cm. At the normal operating voltages this corresponded to 
maximum drift times of 200 nanoseconds. Each plane of a doublet was offset by half a cell with respect to the other plane. This is the standard method used to resolve the left-right ambiguity, that is, the uncertainty about which side of the wire the charged particle passed.

Amplifier and discriminator electronics for the drift wires were mounted in racks on top of each chamber. Signals from the sense wires were amplified and shaped and the resulting ECL level outputs were transmitted via 64 element . cables(Ansley C1622-130) to the experimental trailer. Here they were converted to digital signals by Lecroy Research Services Model 2770A Drift Chamber Digitizers. A block diagram of the electronics used to record drift chamber information is shown in Figure 12.

Each of the Lecroy digitizers contained 96 8-bit time-to-digital conversters (TDC) in a triple width CAMAC module. The TDC's were common stop, meaning that conversion of each signal started at the leading edge and ended with a common stop puise, in this case the experimental trigger. If a second input signal arrived before the stop pulse, the clock would reset, and conversion would start again. This was one of the reasons that it was important not to have multiple hits within the 200 nsec conversion time of the TDC. There will be more discussion of this point in section F. 


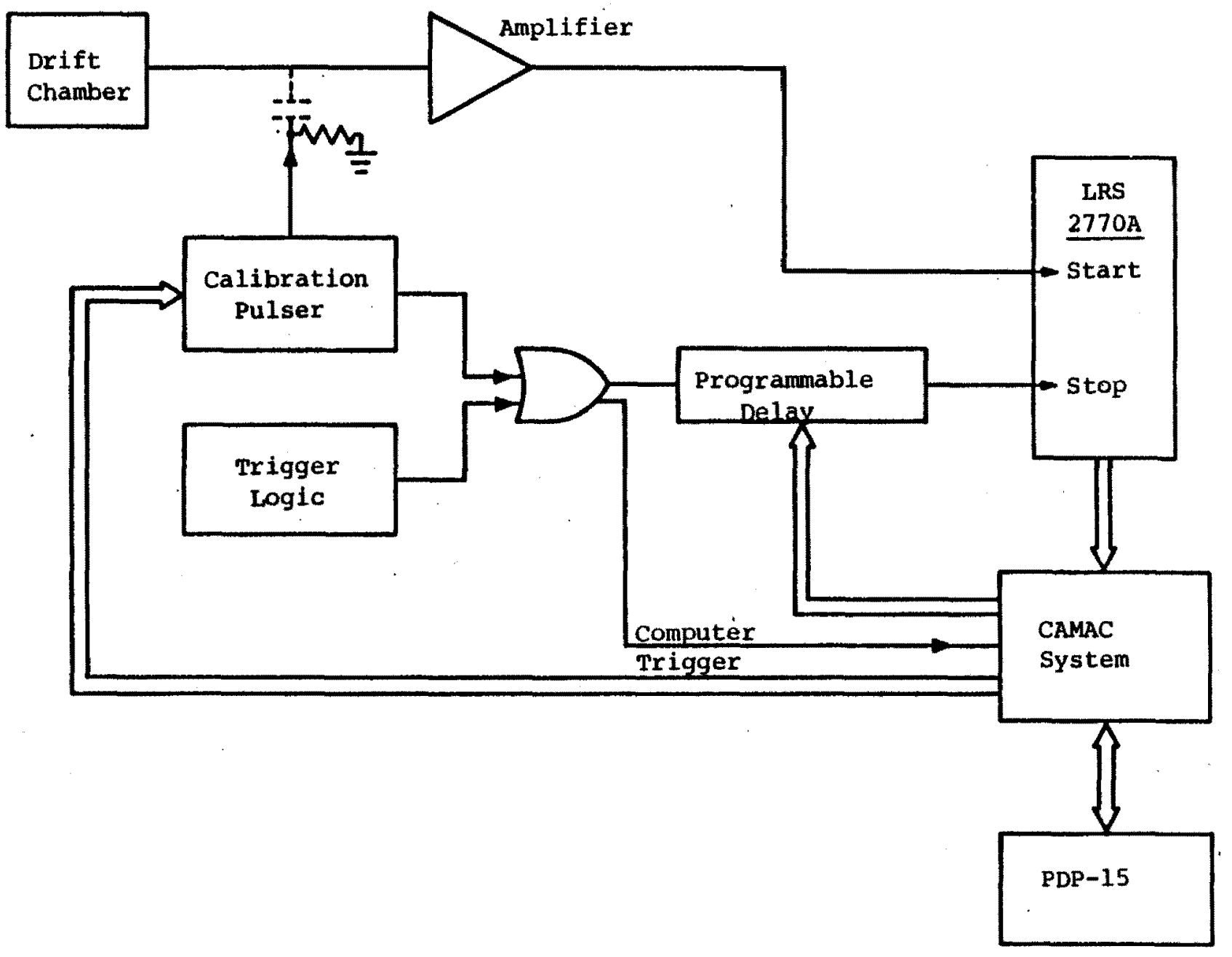


Several time scale ranges were available for the TDC's. The 500 nsec range was chosen because this was well matched to the maximum drift time of $200 \mathrm{nsec}$ for each cell. In this range each count corresponded to approximately 2 nsec, leading to an intrinsic position resolution of about $0.1 \mathrm{~mm}$.

The time distributions for data were set to fall in the middle of this 500 nsec range through a programmable delay unit which could be adjusted from a computer console.(See Figure 12) The ends of the range were avoided because of possible nonlinearities. By an adjustment of this delay with respect to a calibration start pulse, the gains and offsets of each TDC channel were measured. Between spills, the on-line program cycled the delay through. 4 different values, then reset the delay to the value used for data taking. A least squares fit to these 4 different points was used to calculate the gains and offsets. By use of the gains and offsets, the digital counts of the TDC's were converted to times relative to the trigger pulse.

six pulser modules, computer controlled, were used to provide the pulses for between spill calibration of the TDC channels. Each module had 16 output lines and each output line was coupled to 8 amplifier channels. This was done by placing a twisted piece of insulated (number 18) wire across 
the signal lines of the drift chamber amplifiers. The wire was terminated through 50 ohms to the ground plane of the amplifier. Because the on-line progran was not set up to read out all 1000 channels at the same time, only a selected number of the TDC channels were read out for a particular calibration trigger. A more detailed description on the operation of the drift chambers and the TDC's can be found in Reference [34].

\section{2 Photons}

In the planning of this experiment it was calculated that, for photons, an energy resolution of $20 \% / \sqrt{ }$ and a position resolution of $1.5 \mathrm{~mm}$ was necessary to achieve the physics goals. [35] Additional requirements on the photon

detector were: that it be able to handle high rates $\left(10^{5}\right.$ photons/second), have a uniform response over its active area, have good photon-hadron discrimination, and be able to provide selective triggers. The above requirements dictated the use of a Iiquid argon calorimeter(LAC). Since its introduction in the early 1970's [36], the liquid argon calorimeter has proved to be a very useful tool in high energy physics. Our experiment marked one of the first times that a LAC was used for measuring high energy photons. 
An exploded view of the LAC is shown in Figure 13. It consisted of 61 lead sheets and 62 copper-clad G-10 boards. The lead sheets ( $2 \mathrm{~mm}$ thick) were separated on either side from the copper-clad G-10 boards by $2 \mathrm{~mm}$ gaps of liquid argon. The gap was defined by G-10 spacers along the edges of the detector and by small G-10 pins in the interior. The pins were found to be essential to ensure homogeneity of the gap over the entire face of the detector. The detector was held together by stainless steel rods, which were attached to the support structure. When immersed in liquid argon, the detector presented 25 radiation lengths and 1.0 absorption lengths to incident pions.

The copper-clad G-10 boards $1.6 \mathrm{~mm}$ G-10 and $.025 \mathrm{~mm}$ copper) were etched to form strips with $1.27 \mathrm{~cm}$ pitch and 0.5 mm interstrip gaps. The strips were aligned along the $x$ and $Y$ directions on alternate boards. The detector was divided into two sections, front and back, each containing 31 readout boards. The $x$ strips that measured the same coordinate in each section were ganged together and read out by a single amplifier. The $Y$ boards, in addition to the front/back division were separated electronically into left and $r$ ight halfs. Again, the strips for the same $Y$ coordinate in each section were grouped together to be read out by a single amplifier. There were a total of 480 amplifier channels: 224 for $X$ and 256 for $Y$. 


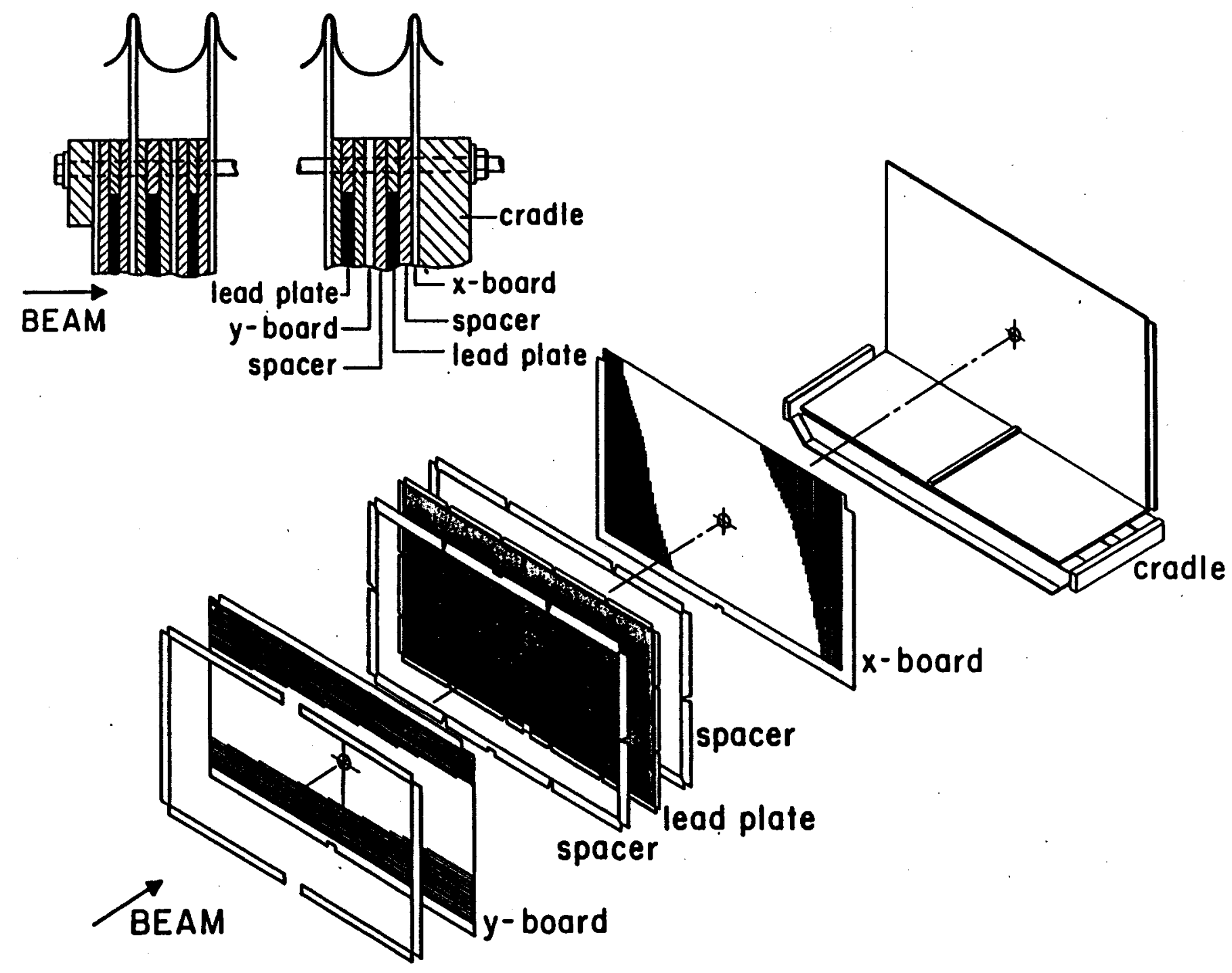


The active area of the detector was $0.81 \mathrm{~m}$ vertically by $1.4 \mathrm{~m}$ horizontally. Each lead plate and G-10 board had a $1.9 \mathrm{~cm}$ hole drilled in the middle to allow the beam to pass through. To further minimize interactions, an evacuated quartz tube was placed in this volume.

The detector support consisted of a welded aluminum cradle with $1.3 \mathrm{~cm}$ of G-10 beneath and in back of the detector. Detector and cradle were suspended by 4 stainless steel rods from a flat stainless steel cover plate. The cover plate was bolted to the top flange of the cylindrical cryostat.(See Figure 14) An 0-ring gasket maintained the seal. The vacuum insulated cryostat was supported by a steel structure (the "tower") which also provided the means for raising and lowering the detector. The tower also provided support for a catwalk around the edges of the cover plate. The catwalk was used in testing of the LAC electronics even when beam was on. This last feature proved to be indispensable in the debugging of the electronics. The whole tower structure could be moved across the floor on airpads. This allowed the beam to be swept across the detector during calibration. (See Section A, Chapter III)

The cylindrical shape of the cryostat left a large space between the front face of the detector and the inside wall of the cryostat. If this region were filled with liquid 


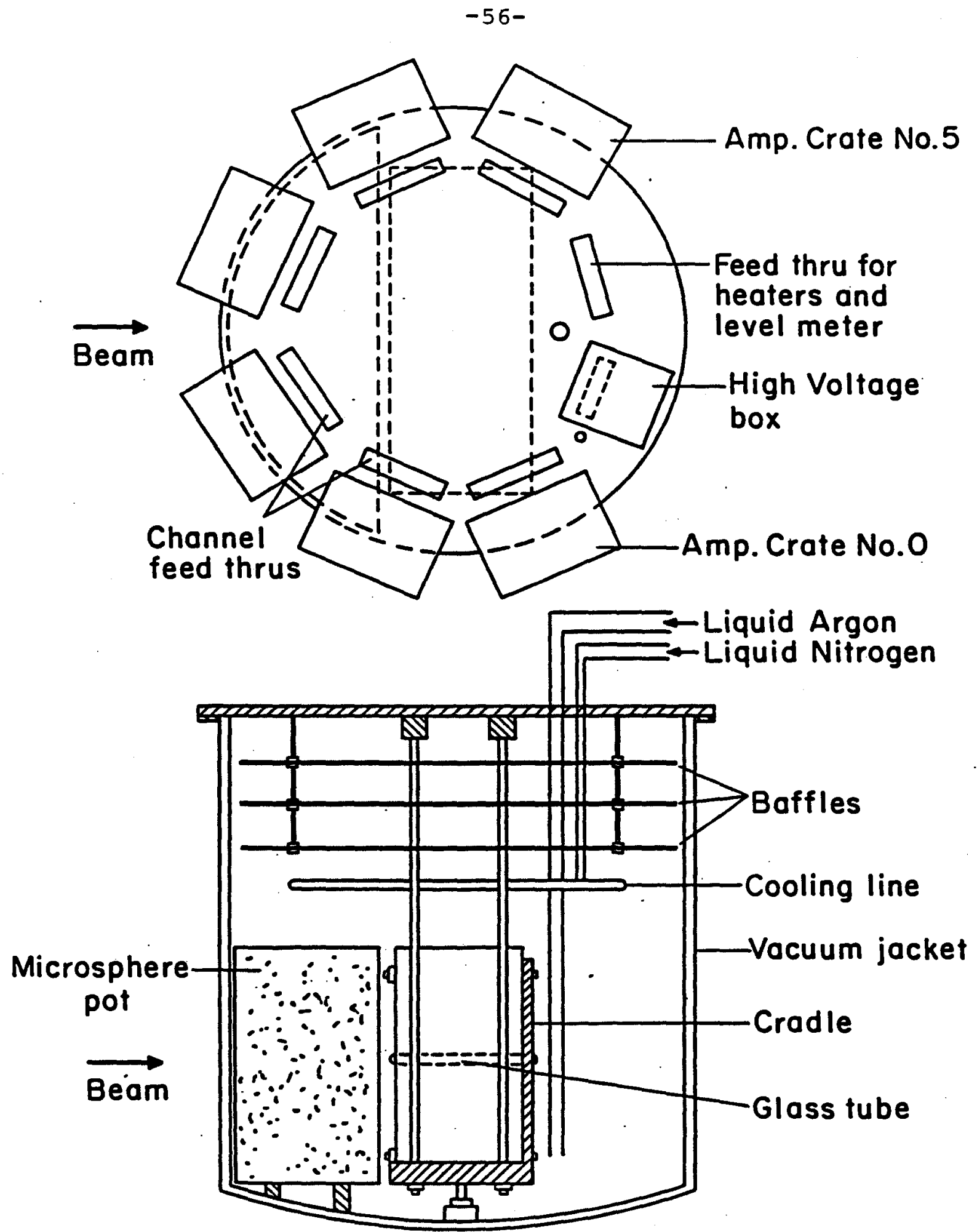

Figure 14 
argon, there would be a serious degradation of energy resolution due to photon conversions and subsequent energy losses. Therefore a thin-walled pot was placed in this section to displace the liquid argon. The pot was filled with hollow glass microspheres(density of $.08 \mathrm{gm} / \mathrm{cc}$ ) to prevent it from imploding when it was pumped down. The walls of the eryostat, the walls of the microsphere pot and about 3 $\mathrm{cm}$ of argon in front of the detector presented a total of about 0.9 radiation lengths.

About 3000 liters of liquid argon were sufficient to cover the detector. The argon was purchased from a local welding supply house in 200 liter dewars. Oxygen contamination, initially 1-4 parts per million, was reduced by passing the argon gas through the purification system shown in Figure 15. In the system, room temperature argon gas was passed over a palladium catalyst along with a small quantity of hydrogen. The resulting water molecules were removed by passing the gas through dessicants and then through a molecular sieve operating at dry ice temperature. Oxygen content at the output of the purification system was typically a few tenths of a part per million. The normal course of operation was to liquify the purified gas into a storage dewar, then transfer the liquid into the cryostat. Tests of the argon showed that even after periods of several months, the oxygen content remained below 1 part per million. 


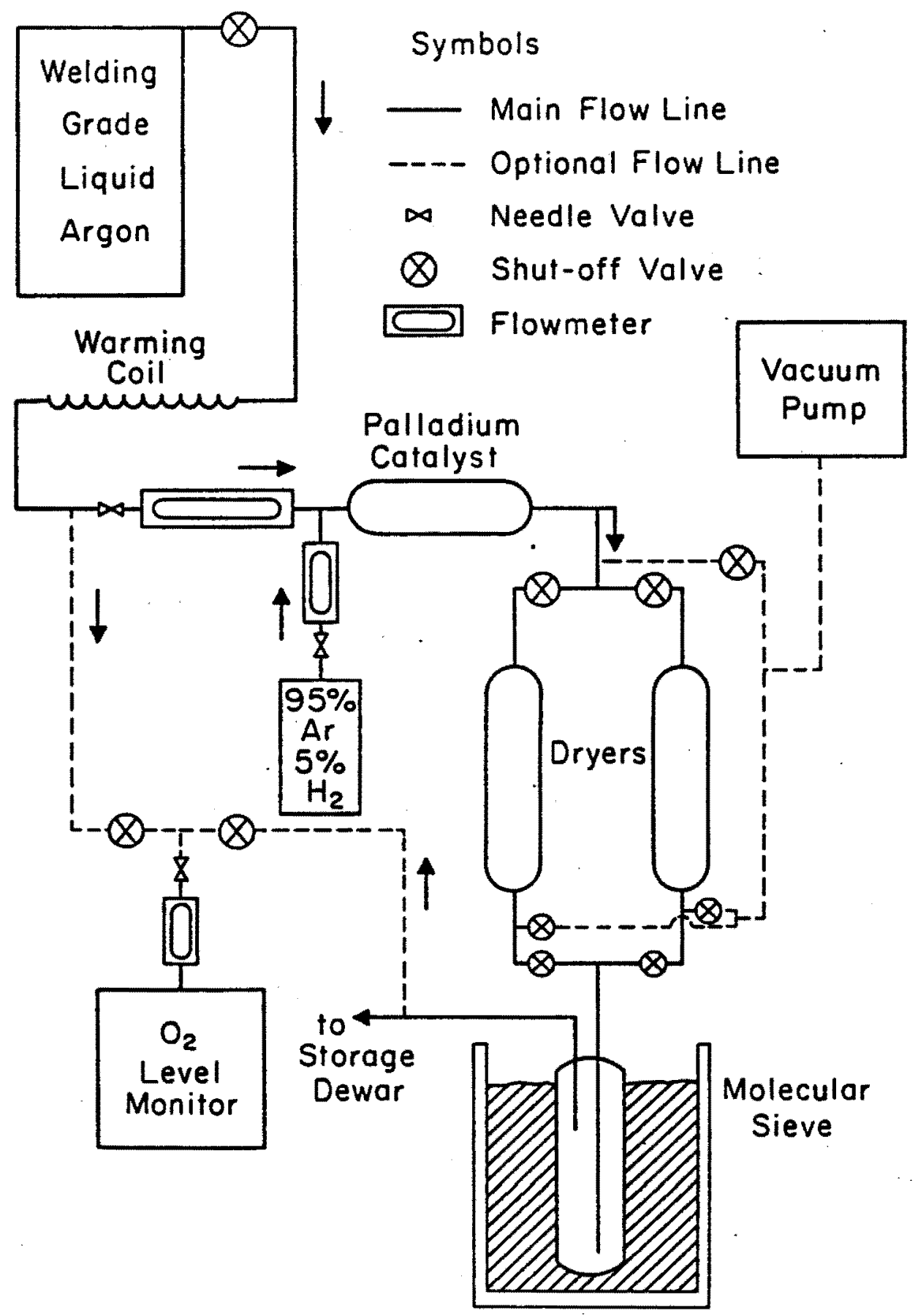

Figure 15 
Studies have shown [37] that the amount of collected charge falls exponentially with the oxygen content. Because of this it was important to be able to purify the argon and to have it remain pure during the course of the running. (See however Section C, Chapter III and Appendix A)

Thermal resistors mounted on the cradle made it possible to sense the level of the liquid. In addition, the resistors helped ensure that there was never more than a $10^{\circ} \mathrm{C}$ temperature gradient across the detector during cooldown. The thermal resistors were connected via twisted pair cable to feedthroughs in the cover plate. Twelve 100 watt resistors, also attached to the cradle, could be used to speed up warming during transfer of the liquid argon out of the cryostat.

A pressure sensitive mercury switch, which controlled the flow of liquid nitrogen through internal cooling coils, was used to regulate the argon gas pressure inside the dewar. During normal operation the pressure varied between 2 and 5 psi above atmosphere, with a cycling period of about 30 minutes.

As mentioned earlier in this section, a high photon rate $\left(10^{5} / \mathrm{sec}\right)$ was expected in the LAC. The strips near the beam hole would be hit most frequently $\left(5 \times 10^{4} / \mathrm{sec}\right)$. Past 
amplifier schemes for liquid argon calorimeters [38] have employed optimal transformer coupling to reduce the noise. In these schemes the detector is impedance matched to the amplifier, with a resultant low noise level. However, due to the narrow bandwidth of transformers, amplifiers employing transformer coupling tend to ring, leading to poor two pulse resolution. In an experiment such as E272 where the photon energies were large ( $5 \mathrm{GeV}$ to $100 \mathrm{GeV}$ ), the noise requirements were not as stringent. The crucial factor was the two-pulse resolving time. It was desired to have a two-pulse resolving time of the order of the charge collection time(400 nsec), but still keep the noise level low enough so that a measurement of 1 GeV/channel could be possible without seriously degrading the resolution. This coiresponded to an rms noise level of less than $10^{-14}$ Coulombs. Both of these criteria were satisfied in the scheme outlined below. (For a more detailed description see Reference [39]).

Figure 16 shows the block diagram of the amplifier electronics for a single channel. Each channel of the LAC, with a capacitance of 2.5 nanofarads(2.2 nanofarads for the Y-strips), was connected to a single amplifier through a multiconnector flat cable. A $10 \mathrm{ohm}$ resistor was used to back-terminate the amplifier at the detector. This back-termination was implemented to prevent $r$ inging. 
a) Electronics for one channel of LAC

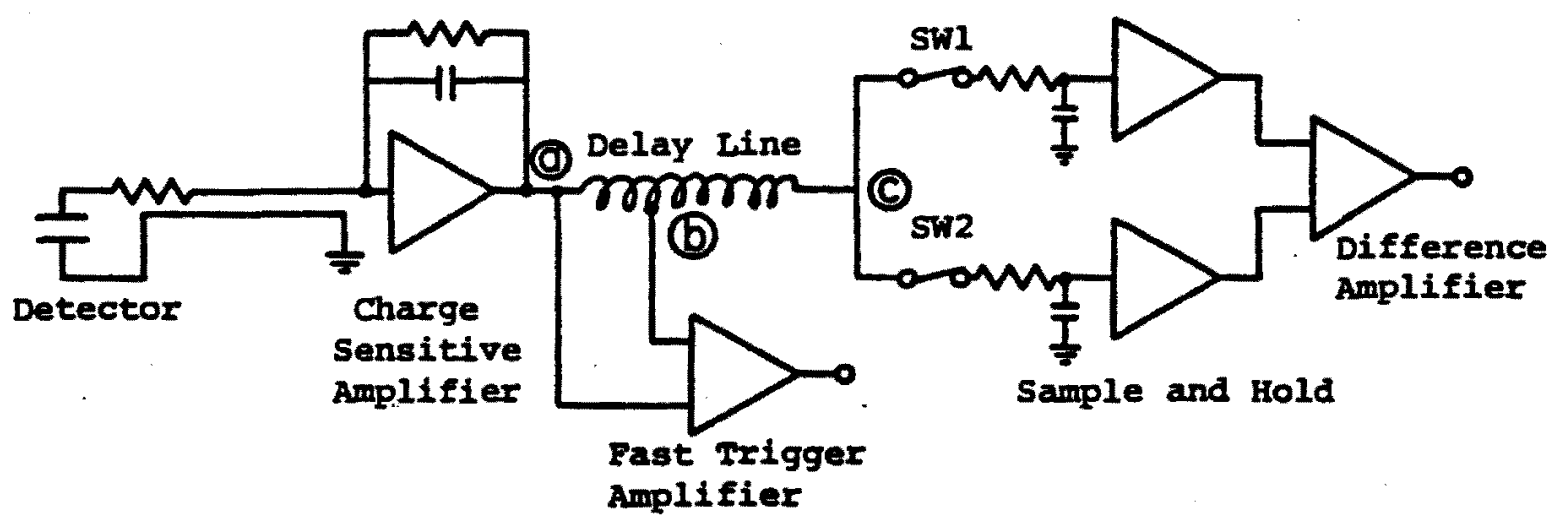

b) Signal at point 0

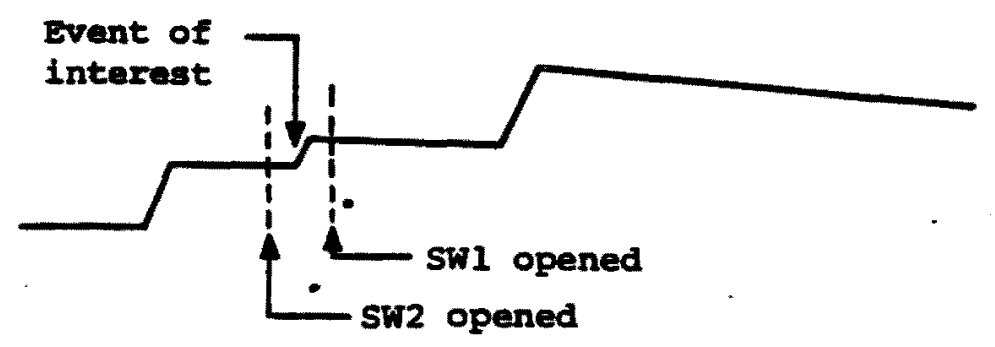

c) Read-out system

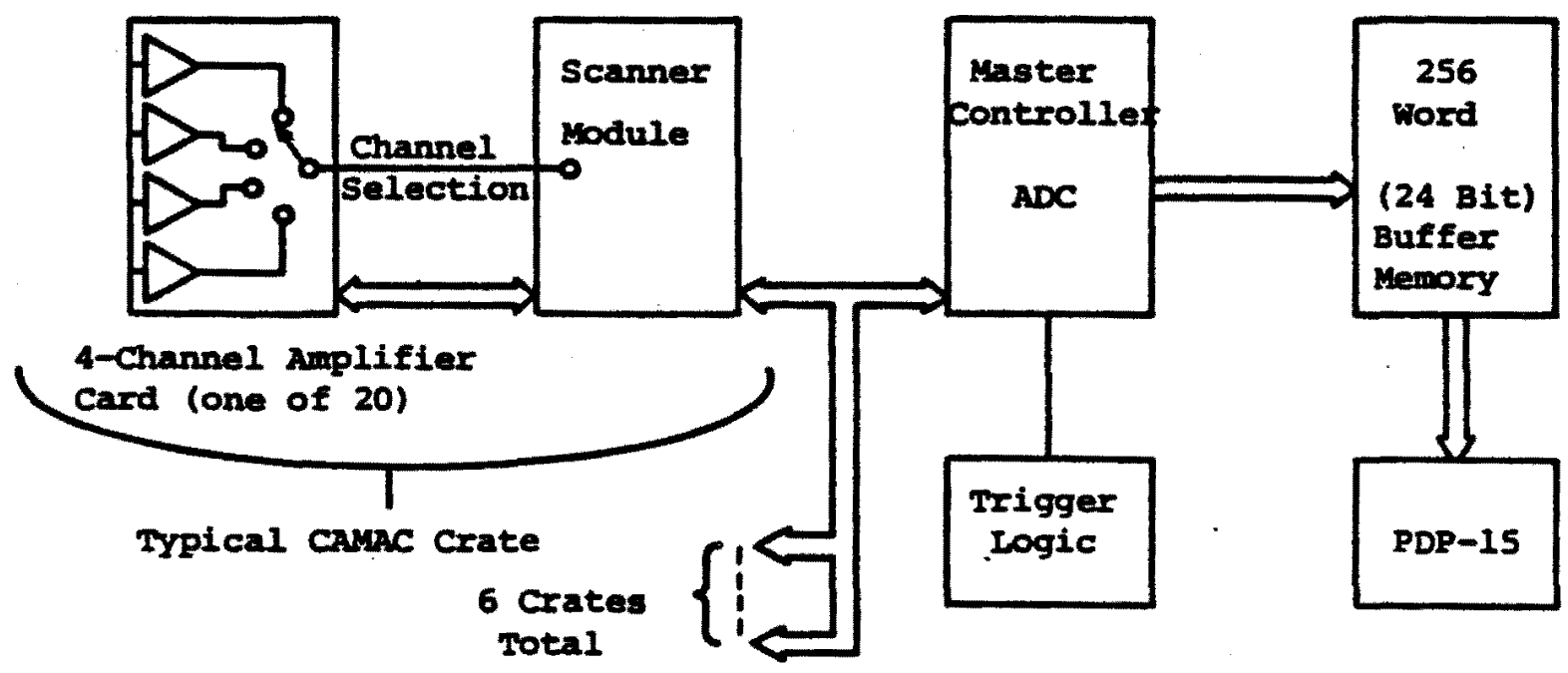

Figure 16 
The amplifier circuit card consisted of a charge-sensitive amplifier, a delay line, a pickoff for a fast-trigger amplifier, two sample-and-hold circuits, a difference amplifier and an output multiplexer. The charge-sensitive amplifier on the front end was capacitively fed back and had a high gain-bandwidth $\left(10^{8} \mathrm{hz}\right)$. The amplifier had a rise time of approximately $120 \mathrm{nsec}$. Because the intrinsic LAC pulse rise time was on the order of 250 nsec, the amplifier response, folded in quadratically, did not have a large effect. Since the amplifier had a large dynamic range, it was possible to make the RC decay time for the amplifier much larger than the rise time. For most channels in the detector the fall time was 200 microseconds, but this was shortened to 20 microseconds for strips near the beam. This was needed due to the larger rates in this region.

The output of the charge-sensitive amplifier passed through a 400 nsec delay line to the sample-and-hold section. The $400 \mathrm{nsec}$ delay provided time for a trigger decision to be made. The cMOs switches sw1 and Sw2 were opened,respectively, before the event of interest, and after the peak pulse height had been reached. With both switches opened the output of the difference amplifier was proportional to the total integrated charge. A DC voltage, adjustable for each channel, was applied to one of the inputs 
of the difference amplifier to provide a pedestal level. This double sampling degrades the signal/noise ratio by a factor of $\sqrt{2}$, but the design figure for the noise level was able to be reached. More importantly, the double sampling feature enabled optimum two pulse resolution.

Another difference amplifier, driven by the front end, provided outputs suitable for triggering purposes. Restoration of the baseline was achieved by delay-line differentiation using a 200 nsec tap in the delay element. A sum of these "fast" outputs provided the energy requirement for the trigger. (See section $F^{*}$ )

The sample-and-hold switches were opened at the appropriate times if the final event trigger was satisfied. The sampled signals were then digitized and read out as shown in Figure 16. Four amplifier channels were placed on one card, and 20 cards, together with a scanner module, were placed in one CAMAC crate. Six CAMAC crates contained the 480 channels in the LAC. The scanner module stepped through the 4 channels in the 20 amplifier cards looking for "sampled-and-held" output levels above a certain readout threshold. For E272 this threshold corresponded to approximately $100 \mathrm{MeV}$. If the output level was greater than this threshold, the scanner would send the analog output from the amplifier to the Master Controller module, along with a 
"Data Available" signal. The controller module then digitized the analog signal and sent the digitized result, along with the channel address, to a 24-bit buffer memory. After digitization of all channels over threshold, the data acquisition computer would read out the buffer memory. The scanning and conversion time for a single crate, with all channels above threshold, was about 2 milliseconds.

A scheme was implemented to keep track of the pedestals and gains of the 480 LAC channels. To measure the gains, a pulse of known amplitude was injected, between spills, into capacitors connected to the signal lines of the channels. The resulting amplifier pulse was digitized and read out as in a normal event. Different sections of the detector could be pulsed independently so as to cut down on the number of channels read out for any particular calibration event. The pedestals were measured by setting the readout threshold to zero and digitizing the amplifier outputs. As will be seen later, the amplifier gains were very stable. The pedestals tended to fluctuate; consequently, a run-by-run monitor of the pedestal levels was needed for reconstruction.

E. Experimental Trigger 
A selective trigger was required to single out the $0.05 \%$ of the total $\pi^{+} \mathrm{Pb}$ cross-section that corresponded to $\rho^{+}$production. The trigger was designed to enhance the presence of coherent interactions with one charged particle and at least one photon in the final state. The thinness of the nuclear targets, required for adequate $t$ resolution(see Section (C), mandated the use of a low-level trigger processor to eliminate interactions that occured downstream of the target. These interactions might otherwise have dominated the trigger rate. An additional low-level processor was used to restrict the triggers to those with only one charged track in the final state. Our experiment marked one of the first uses of trigger processors at Fermilab. [40] More detail will be given later.

Figure 17 shows a schematic diagram of the scintillation counters used in the trigger, and Figure 18 shows a general layout of the trigger logic.

In the vicinity of the target the beam was defined by two scintillation counters: B2 and B3. It was important to have a stable reference time for use with the Lecroy $2770 \mathrm{~A}$ Drift Chamber Digitizers. This reference time was provided by the leading edge of the discriminated B3 signal and all other timings in the trigger were derived from it. Counters BO and Bl,bracketing the Cerenkov region of the beamline, 


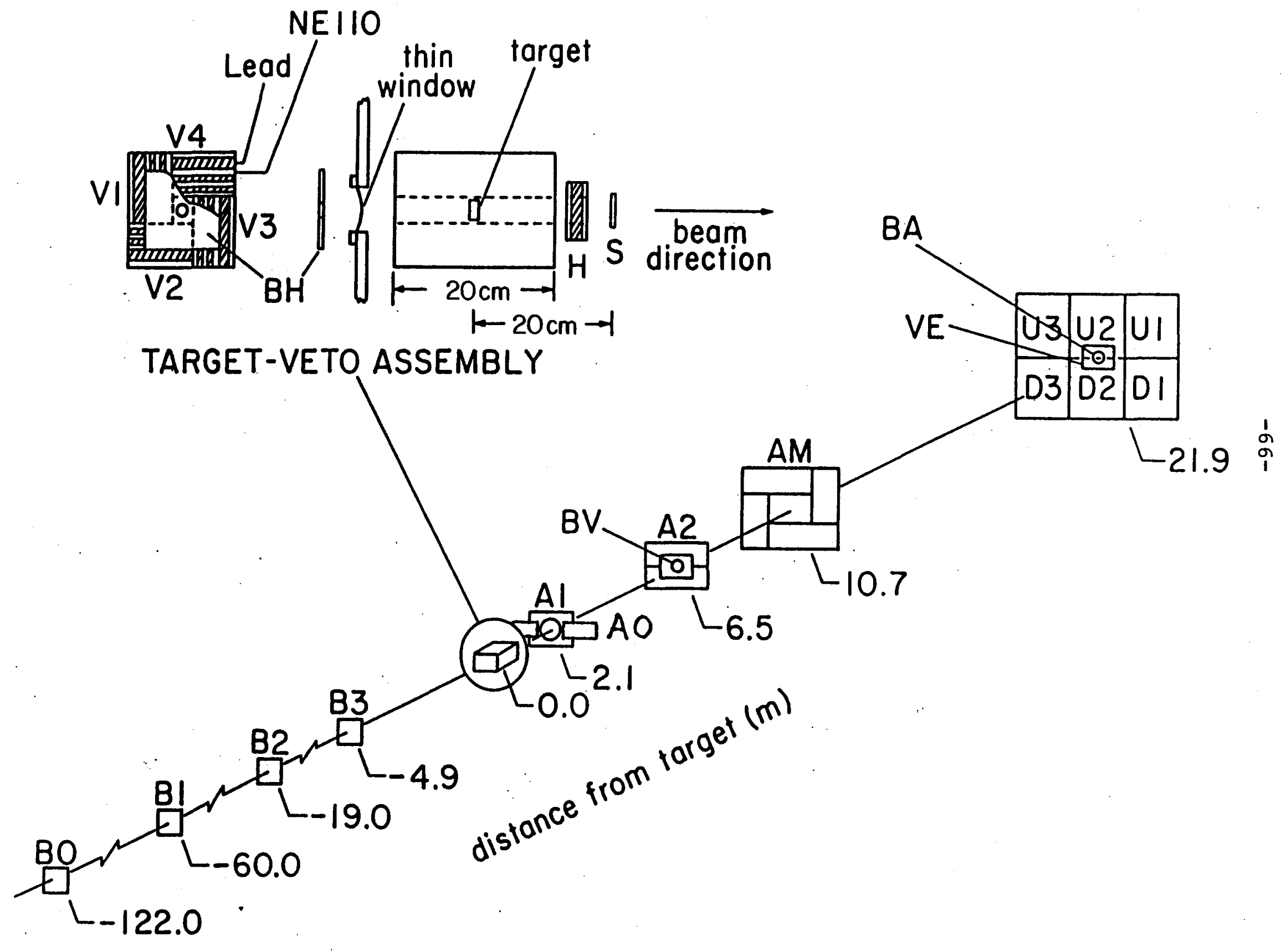




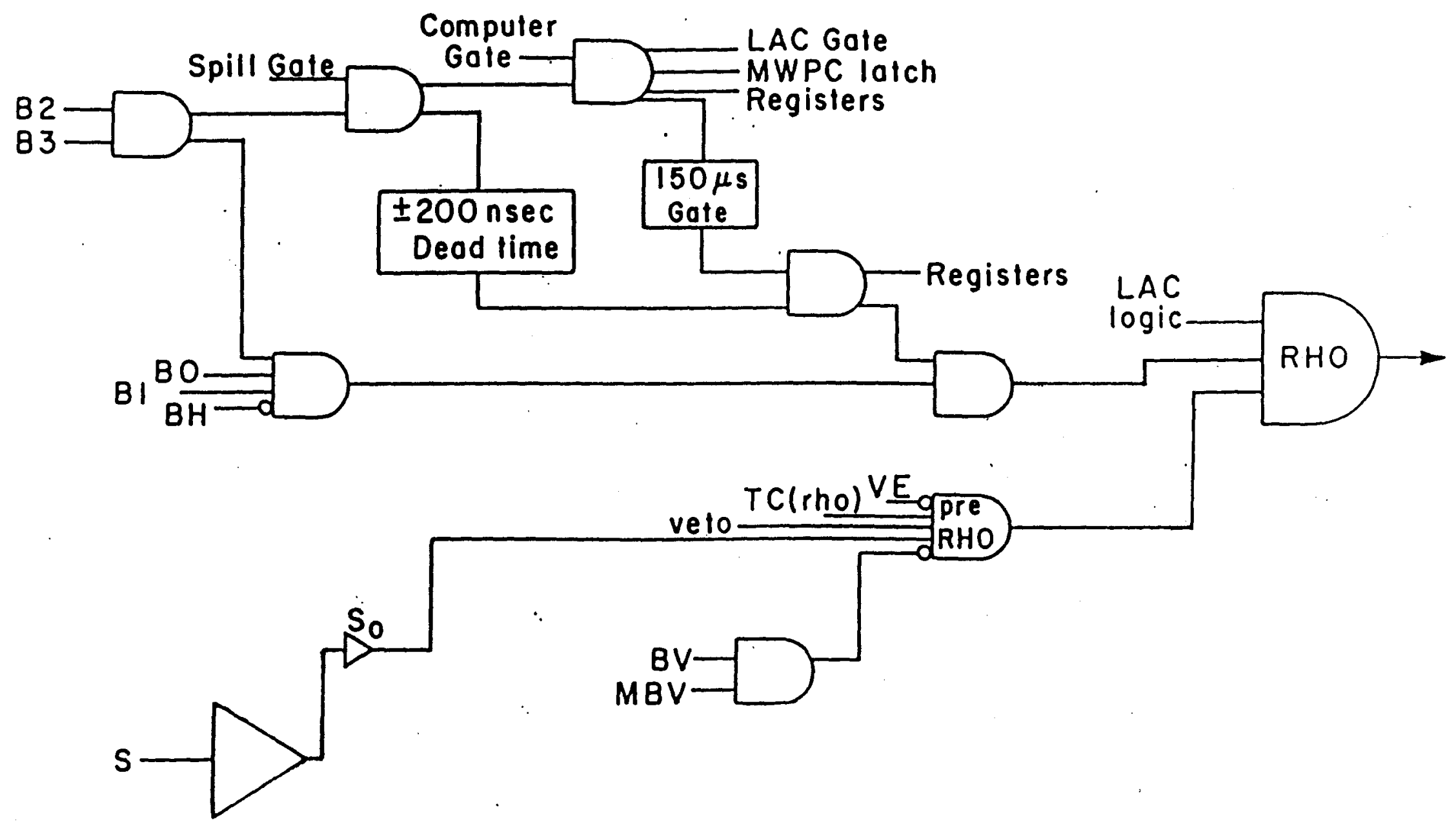


were put in coincidence with the B2 B3 signal to improve the beam definition. A halo counter , BH, located upstream of the target, that had a $1.6 \mathrm{~cm}$ diameter hole at its center was used in veto. This ensured that the defined beam track intersected the target. The beam definition was thus: $\mathrm{B}=\mathrm{B} 0 \cdot \mathrm{BI} \cdot \mathrm{B} 2 \cdot \mathrm{B} 3 \cdot \mathrm{BH}$. Signals from Cerenkov counters were put in coincidence with the beam to define the incident particle type. Thus the coincidence B.KI signaled an incident kaon. The coincidence $B \cdot K 2 \cdot K I$ signaled an incident pion. It was necessary to veto on the $\mathrm{Kl}$ signal in defining pions because of the presence of kaon contamination in the $\mathrm{K} 2$ signal.

As mentioned earlier, the $2 \mathrm{~cm}$ cell size for the drift chambers corresponded to a maximum drift time of 200 nsec. To prevent confusion from spurious hits in the drift chambers, caused by events other than the one of interest, the beam particles were required to be at least 200 nsec apart. This was accomplished by implementing a \pm 200 nsec deadtime around the $\mathrm{B} 2 \cdot \mathrm{B} 3$ coincidence. At rates typical for this experiment, namely, $3 \times 10^{5} /$ second, this caused a deadtime of about 108 .

Accelerator reference times were provided corresponding to the beginning and end of spill. The trigger logic was gated on during this time. The B2 B 3 coincidence, gated by the spill gate and by the computer ready gate, 
provided a fast signal that could be used to strobe the LAC and to provide a latch for the proportional chamber signals. The gated $\mathrm{B} 2 \cdot \mathrm{B} 3$ signal, in coincidence with $\mathrm{B} 0 \cdot \mathrm{B} 1 \cdot \mathrm{B} 2 \cdot \mathrm{B} 3 \cdot \overline{\mathrm{BH}}$, provided the normalized beam count.

An array of counters was arranged around the target as shown in Figure 17. In coherent interactions, the nucleus recoils with a momentum of less.than $100 \mathrm{MeV} / \mathrm{c}$. This momentum is not high enough for the nucleus to leave the target. A good test for coherence, then', is the absence of any large angle recoil particles. The V1-V4 and $H$ counters were used as veto in the trigger logic. The $4 \mathrm{~V}$ counters consisted of sheets of NEllo scintillator interleaved with lead plates for a total of 5.2 radiation lengths. The H counter, located downstream of the target, was composed of a sandwich of lead and scintillator with a $3.2 \mathrm{~cm}$ diameter hole in the center. The light from $\mathrm{V}$ and $\mathrm{H}$ counters was viewed, via plastic light pipes, by Amperex 56AVP phototubes. To provide good two-pulse resolution the outputs were clipped with 4 nsec shorting cables. To decrease vetos caused by delta rays in otherwise good events, the inner walls of the $V$ counters were lined with $0.25 \mathrm{~mm}$ of copper.

Various veto counters, placed in the upstream half of the spectrometer (shown in Figure 17), defined the geometrical acceptance of the experiment(essentially the magnet 
aperture). The AO, A1, A2, and AM counters were scintillator-lead sandwiches, about 6 radiation lengths thick, with RCA 8575 phototubes. Two scintillation counters were placed, in addition, along the inside vertical faces of the BM109 magnet. These counters, also with 8575 phototubes, were used to veto events in which low-momentum charged particles of either sign were bent into one of the faces of the magnet.

Because the targets were at most a few percent of an interaction length thick, most of the beam passed through them without interacting. A small counter, BA, $12.9 \mathrm{~cm}$ in diameter and $0.16 \mathrm{~cm}$ thick) was placed in front of the LAC and used to veto events with charged particles in the beam region.

The signals from all of the veto counters in the experiment were fanned together to form a common veto logic signal:

$\mathrm{B} 2 \cdot \mathrm{B} 3 \cdot \mathrm{BH} \cdot \overline{\mathrm{BA}} \cdot(\overline{\mathrm{V} 1+\mathrm{V} 2+\mathrm{V} 3+\mathrm{V} 4}) \cdot(\overline{\mathrm{A} 0+\mathrm{A} 1+\mathrm{A} 2+\mathrm{A} 3+\mathrm{AM}+\mathrm{B}})$

This signal formed part of the Rho trigger logic.

The number of charged particles that left the target was determined by a thin $(3.8 \mathrm{~cm} x \quad 3.8 \mathrm{~cm} \times 0.16 \mathrm{~cm})$ 
scintillation counter located $20 \mathrm{~cm}$ downstream of the target. A discriminator level $s_{0}$, corresponding to one or more charged particle, was required for the Rho trigger. The pulse from this $S$ counter was ADC'd for use in later offline analysis.

The material in the beamline downstream of the target amounted to .024 pion interaction lengths. This was comparable to or greater than the number of interaction lengths in any of the targets. Without some protection, the trigger rate would be dominated by these downstream interactions. To suppress these events, a matrix was formed from signals of one $X$ plane in each of the $J 1$ and $J 2$ chambers and from the signals in the $3 \mathrm{~cm}$ central beam region of both of the PIX chamber planes. The beam hits in $J 1$ and $J 2$ were used to define an incident track direction. If any hits were present in the $\mathrm{P} 1 \mathrm{X}$ planes at an angle of less than $0.3 \mathrm{mrad}$ relative to the incident beam direction, the Matrix Beam Veto(MBV) was satisfied and the event was rejected. If set up in this manner, the MBV would have rejected events that had charged particles produced at large angles in $Y$ but small in $x$. This was so because only the $x$ proportional coordinates were used in forming the matrix. To restrict the vetoing to small angles in both $Y$ and in $X$, the MBV signal was put in coincidence with the signal from a small scintillation counter BV(2.5 cm diameter and $0.16 \mathrm{~cm}$ thick). 
$B V$ was placed in the beam region just downstream of PlX. The resulting coincidence was called $M(B V)^{2}$. The efficiency of the $M(B V)^{2}$ was found to be about 908 and was limited mainly by the inefficiencies of the proportional chambers.

A high speed track counting system (Bellcord) was developed [41] to provide a fast decision on track multiplicities in the proportional chambers. The system makes use of coaxial cable "buses" on which fast pulses of the proportional chambers P1X, P1Y, and P2 were summed. signals from the two staggered planes were interleaved to reduce the effective spacing by a factor of 2 . A track was defined in a chamber by the presence of a contiguous group of "hit" wires with at least one signal-less wire between groups. The Bellcord provided logic levels for each chamber(within a time of 60 nsec) corresponding to $1,2,3,4$ or 5 tracks. The signals were latched for offline comparison with the MWPC information and were found to be in agreement more than 908 of the time. The track counting requirements for the Rho trigger(TC(Rho)) were that 1 or 2 tracks were found in the PIX-PIY system and 1 or 2 tracks were found in P2. The requirements were kept loose to allow for noise in the chambers, delta rays, and in the case of $P 2$, the possibility of large angle tracks downstream of the magnet. 
The LAC played a prominent role in the Rho trigger. The most serious background to the trigger came from elastic scattering of the incident pions. The angle of scattering could have been large enough so that the $M(B V)^{2}$ was not satisfied, yet small enough to stay within the geometrical acceptance of the spectrometer. The energy deposition in the LAC, although different in character from that due to photons,was occasionally large enough to satisfy any reasonable energy requirement for the trigger. Because of the large cross-section, elastic scattering events could have completely dominated the trigger rate. suppression of such events was achieved by making use of the kinematics of $\rho^{+}$ production and decay. Because $\rho^{+}$production takes place at small $t$, the momentum vector of the $\rho^{+}$lies essentially along the beam axis. If the charged pion from the $\rho^{+}$decay was produced above the center plane of the detector, kinematics required the $\pi^{\circ}$ to be emitted below the center plane.

Two energy sums(EUP and EDOWN) were formed from the fast outputs of the $Y$ amplifiers in the front half of the detector. As their names imply, EUP was the sum of the outputs of the amplifiers above the center line and EDOWN the sum of those below. The EUP and EDOWN analog signals were fed into discriminators which had thresholds corresponding to an energy of roughly $7.5 \mathrm{Gev}$. Six scintillation counters, 3 UP and 3 DOWN, were mounted on the front of the tower, 
covering the entire area of the LAC. In order for the trigger to be satisfied, a signal from the EUP discriminator could not be accompanied by any signals from the UP scintillation counters, and similarly for EDOWN. In addition, analog signals for EUP and EDOWN were fanned together into a discriminator with a threshold corresponding roughly to $10 \mathrm{Gev}$. This formed a total energy requirement for the event, ETOT. A schematic of the LAC logic is shown in Figure' 19.

A background from elastic scattering still remained for events where the scattering occurred in the median plane of the LAC. Energy could be deposited in the LAC in such a way as to satisfy the EUP or EDOWN (and ETOT) requirements, without firing the corresponding scintillation counters. This background was suppressed by the VE counter $(5 \mathrm{~cm}$ vertically by $10 \mathrm{~cm}$ horizontally and $0.16 \mathrm{~cm}$ thick) which was. placed to cover the beam region near the median plane. The use of the VE counter as a veto caused only a small loss of good events( calculable by Monte Carlo), but almost totally eliminated the remaining elastic scatters.

In summary, the definition of the Rho trigger was:

$$
\text { Rho=B } \cdot\left(\text { Veto LogiC) } \cdot S_{O} \cdot T C(R h o) \cdot \overline{M(B V)}^{2} \cdot \nabla E \cdot(L A C \text { LogiC) }\right.
$$




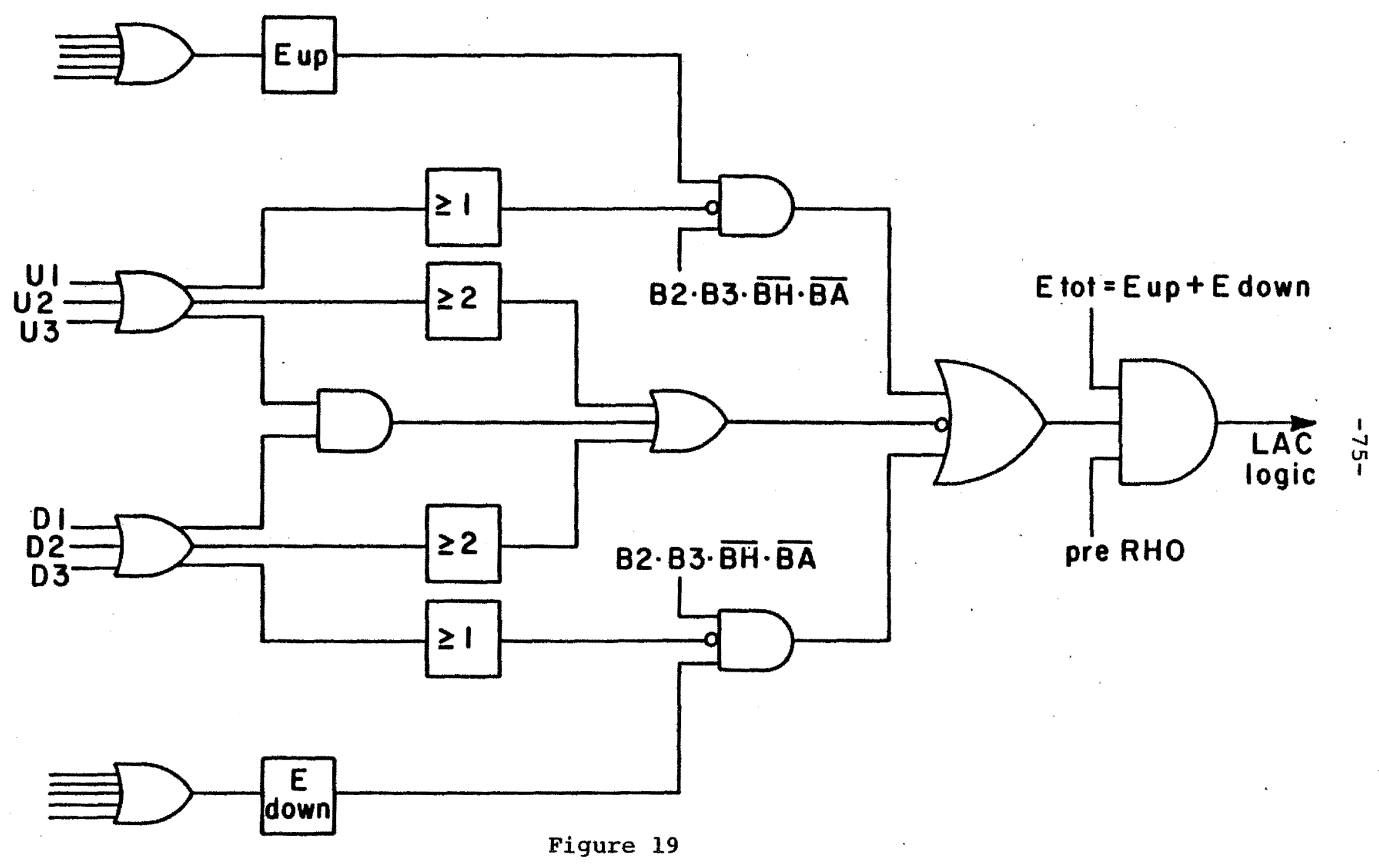


Various minimum bias beam triggers were also taken to help with the normalization and the understanding of systematic effects in the spectrometer. Their definition can be summarized essentially as below:

$\mathrm{Beam}=\mathrm{B} \cdot \mathrm{B} 2 \cdot \mathrm{B} 3 \cdot \mathrm{BA} \cdot \mathrm{BE}$

with the magnet on and

$\mathrm{Beam}=\mathrm{B} \cdot \mathrm{B} 2 \cdot \mathrm{B} 3 \cdot \mathrm{BH}$

with the magnet off.

F. Data Acquisition and Monitoring

Whenever the experimental trigger was satisfied, the trigger logic was gated off and various readout signals and gates were generated. Data for the event were digitized and then were read out throught a CAMAC system by the on-line computer, a PDP-15. The system had a total of 6 CAMAC crates controlled by one branch driver and interfaced to the PDP-15. The 6 CAMAC crates contained 11 TDC modules(to digitize the drift chamber information), 2 buffer memories(to record the proportional chamber and LAC information), 2 ADC units(to digitize various analog signals), and various registers and scalers to record beam and counter information. The PDP-15 
parformed some rudimentary analysis on the data and then wrote. it to magnetic tape. A schematic of the data flow is shown in Figure 20.

There existed the possibility of sending a subset of the data to the central Fermilab computer, a Cyber-175, to be analyzed using the experiment's offline programs. This was accomplished by the use of the Bison-Net system [42]. The PDP-11 computer shown in Figure 20 controlled the transfer of data to the system. By the use of this feature both hardware and software details of the experiment could be monitored.

Beam spills were separated by a period of time which varied from 8 seconds to 16 seconds, depending on the time of day. During this period calibration pulses were sent to the drift chambers and to the LAC, and the resultant data written on tape. Problems were searched for and the experimental equipment monitored by on-line analysis of these calibration events along with a sample of data events.

About 300 tapes were written during October and November of 1979. Most of the data were taken with a 200 Gev positive beam, with some time being devoted to a cablibration run at negative $50 \mathrm{GeV}$. Three nuclear targets were used, carbon, copper and lead, with most of the data being contained in the copper and lead running. In addition, 


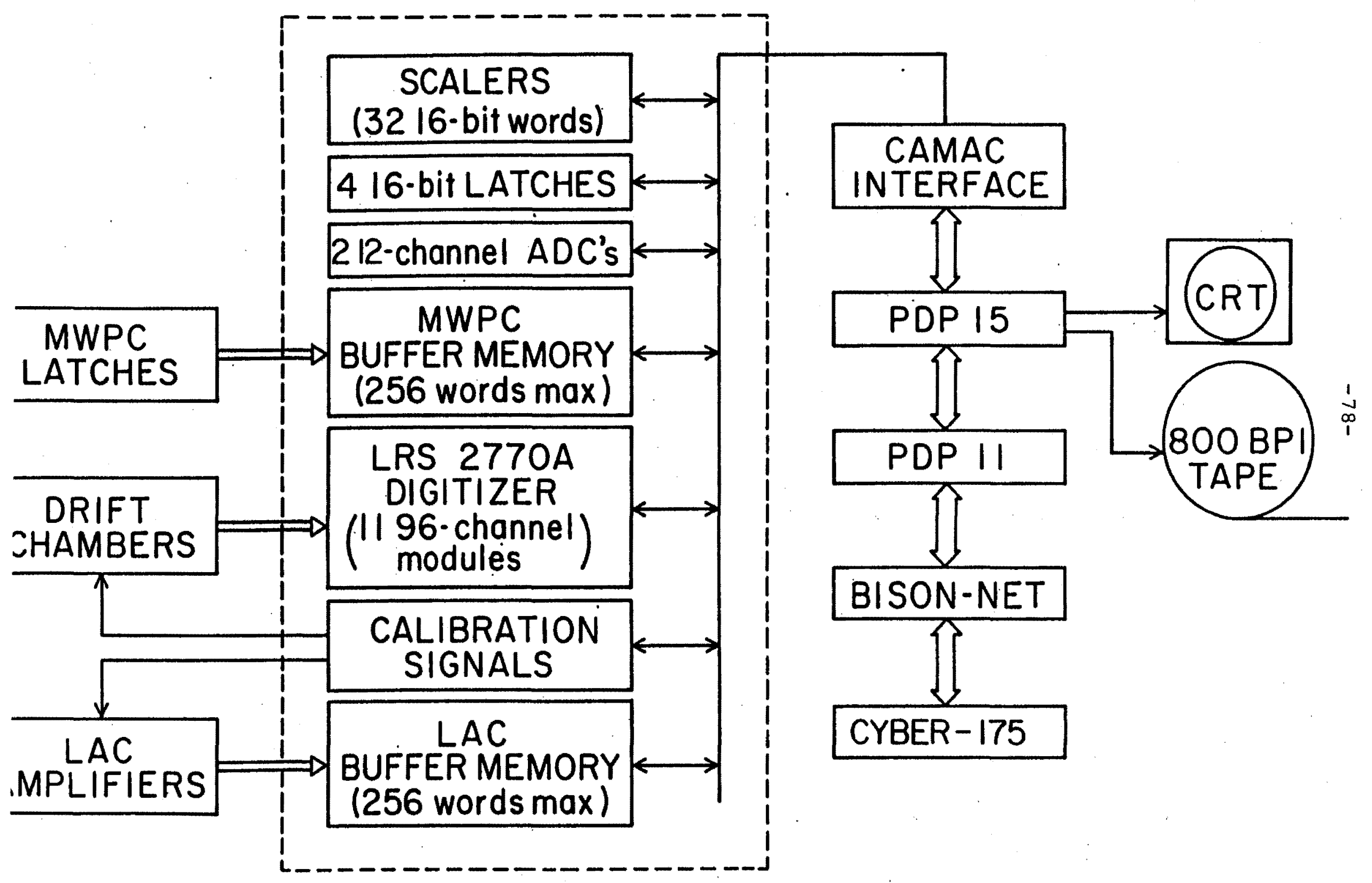


about 108 of the data was taken with no target present. Some straight-thru beam data were also taken with magnet off for alignment of the chambers. 
Chapter III

Data Processing

In this chapter the procedures for calibration and alignment of the spectrometer and for reconstruction of the events will be presented. Section A describes the calibration and alignment of the drift chambers and of the LAC. 'Section B describes the algorithms for track and photon reconstruction while, in. Section $c$, the run-to-run consistency of the experimental data is discussed.

A. Calibration and Alignment

A.I Drift Chambers

Five parameters were necessary to determine the spatial locations of the charged particle "hits" in the drift chambers. Two, the gains and offsets for each TDC channel, were electronic in nature; the other three, arift velocity, sense wire position, and the zero point in the time scale related to the characteristics of the chambers themselves. 
The calibration system for the TDC's was discussed in Chapter II. Four calibration pulses, of known separation in time, were used to trigger the TDC's. Thus, four points were available for each channel to determine the conversion of digital counts to nanoseconds. The four points were fit to a straight line with the slope of the line determining the gain(number of nanonseconds/count) and the zero intercept of the line determining the offset of the TDC channel. The linear fit worked very well; the mean deviation of these four points from the fitted straight line was less than 1 nsec. (One nanosecond corresponded to a drift distance of about 50 microns.) As the total spatial resolution for the drift chambers was on the order of 200 microns, the contribution from the TDC calibration was not significant.

Calibration data were taken in every run for each of the 1034 channels of the Lecroy $2770 \mathrm{~A}$ digitizers used in the experiment. Since the TDC units were sensitive to temperature changes, the gains and offsets tended to fluctuate. (See Reference [34] for more detail.) In Figure 21 are shown the time variations of the gains and offsets of two representative TDC channels. The scale of these fluctuations mandated the use of individual TDC calibration fits for each run. 

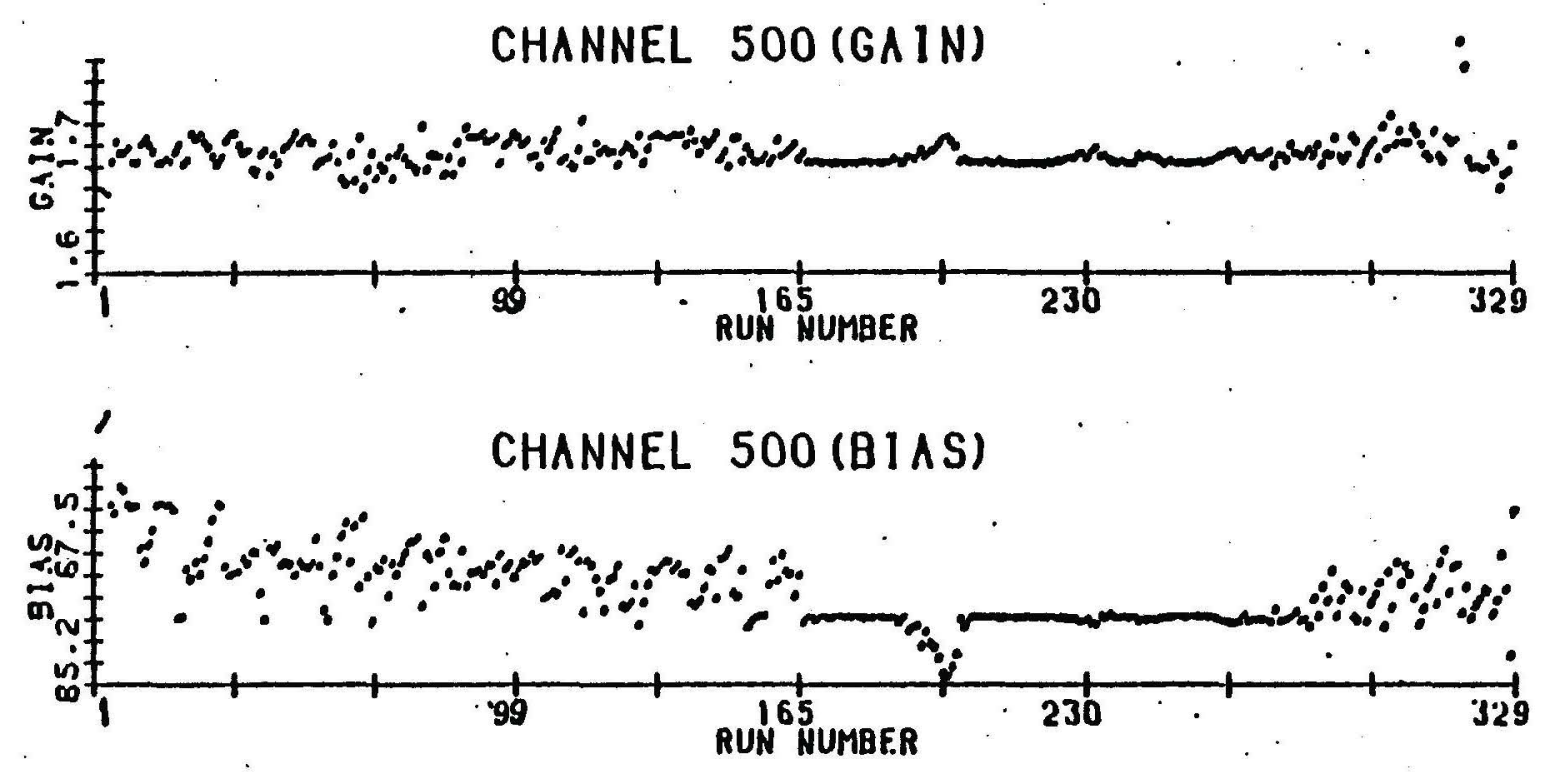

CHANNEL 900 (GAIN)
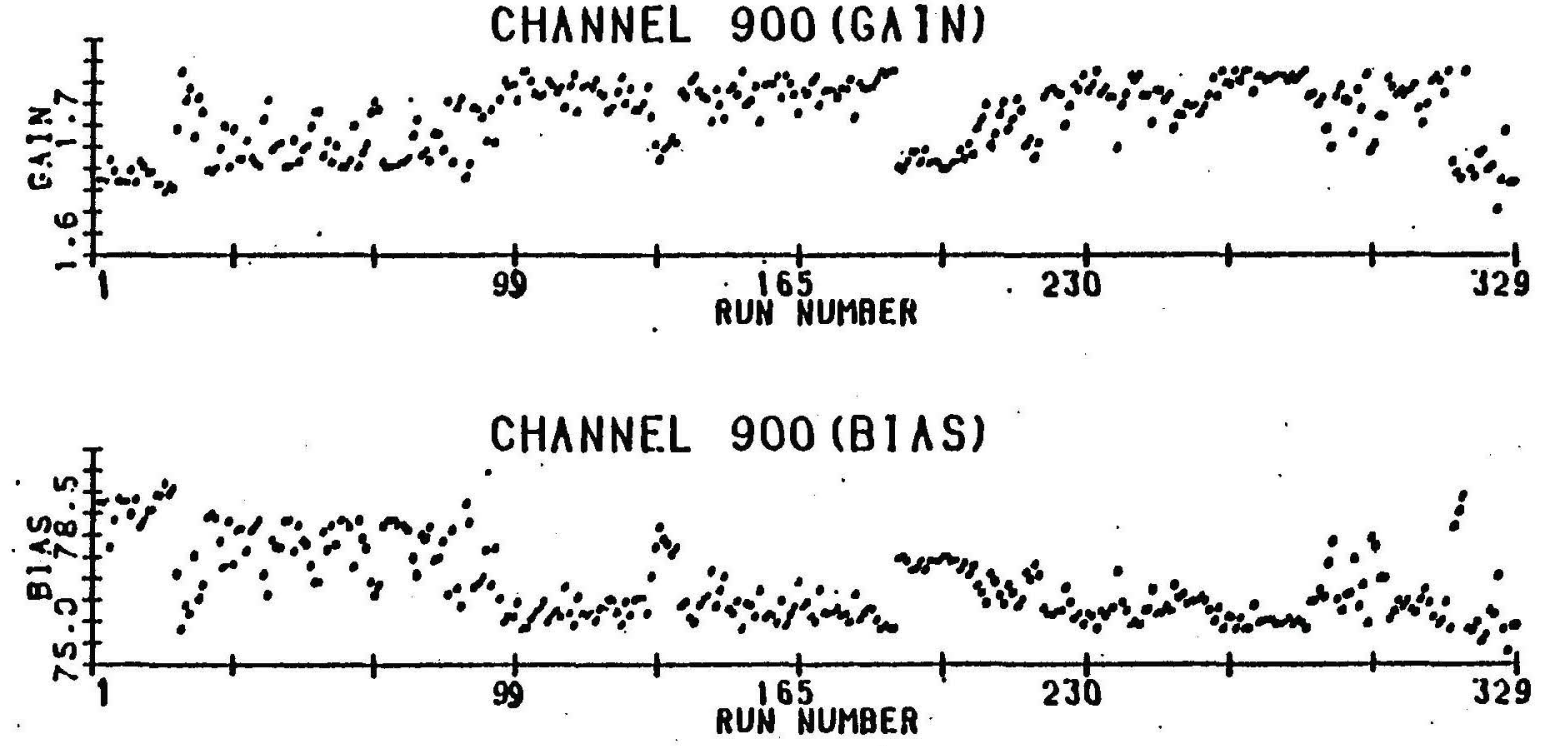

Figure 21 
To convert the time intervals determined by the TDC's into spatial coordinates, it was necessary to know the zero point of the time scale, the drift velocity of the ionization electrons produced along the charged track, and the positions of the sense wires.

"Straight-thru" tracks( beam data taken with no target present and the BM109 off) were used to align the chambers with respect to each other and to the beam axis. The first step was to establish a coordinate system for each event using the data from the beam chambers $\mathrm{Jl}$ and $\mathrm{J} 2$. It was assumed that all chambers were perpendicular to the beam and that all wires formed their design angles with the vertical. Furthermore, it was also assumed that the wire spacing in each plane was uniform. (During construction, sense wire placements were found to be accurate to \pm 30 microns. Again for more detail, see Reference [34].) The use of these assumptions allowed one alignment parameter per plane, the displacement transverse to the wire direction, to completely describe the position. Comparison with the data showed these assumptions to be reasonable.

The cutoff values for the time distributions in the different channels gave an initial idea of the zero point. In a similar way, the widths of the distributions provided an estimate of the drift velocity. In an iterative fit, the 
resolution of the drift chambers was optimized by varying the drift velocities and time offsets along with the alignment parameters. After the fit converged, the alignment parameters were fixed, and the drift velocities and offsets were varied to optimize the resolution in data taken in the normal Rho trigger mode. Rho trigger events covered a larger area of the chambers than did the "straight-thru" events. The values obtained for the drift velocities and time offsets were more realistic in that they were not determined solely by events limited to a narrow region along the beam axis.

For the chambers upstream of the BMI09, the arift velocity was determined to be $5.13 \times 10^{-3} \mathrm{~cm} / \mathrm{nsec}$; for those downstream, the drift velocity was $4.90 \times 10^{-3} \mathrm{~cm} / \mathrm{nsec}$. The difference in drift velocities was due to the downstream chambers having half as many field shaping wires. In Figure 22 are shown plots of TDC times versus position in the drift chamber cell, with the positions being determined by the Jl and $\mathrm{J} 2$ beam chambers. A linear relationship exists except at the edge of the cell.

The spatial resolution for the drift chambers was found to be 200 microns for the upstream chambers and 250 microns for the downstream chambers. close to the beam (within $0.75 \mathrm{~cm}$ ) the resolution was somewhat worse due to the accumulation of space charge. (See Reference [43] for 


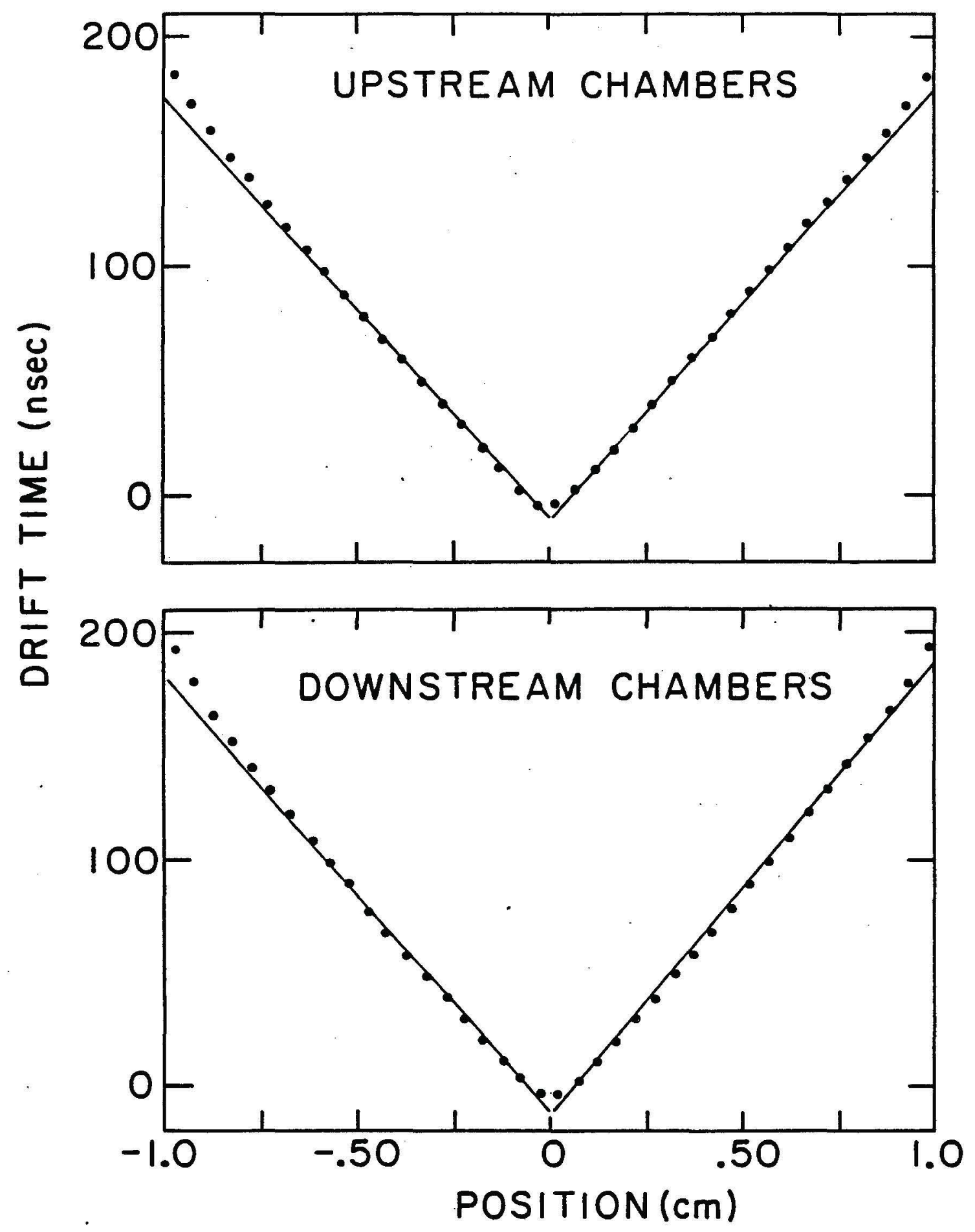

Figure 22 
more detail.) The kinematics of $\mathrm{K}^{+} \rightarrow \pi^{+} \pi^{\circ}$ and $\rho^{+} \rightarrow \pi^{+} \pi^{\circ}$ events, however, were such that, in most cases, the charged pion was outside the beam region. (For example, see Figure 36 in Chapter IV.)

\section{A.2 Liquid Argon Calorimeter}

The calibration of the LAC involved determination of two types of constants: those relating to the electronics and those relating to the actual charge collection. The amplifier pedestals and gains belong to the first type. As mentioned in Chapter II, the pedestals and gains were monitored in between spills calibration events. For every run the average pedestal value for each channel was calculated. Before any data reconstruction took place, this average pedestal value was subtracted from the ADC count measured in each channel. The pedestal value for each amplifier channel was determined by two sources. The major source, the DC offset of the difference amplifier, was measured adequately by the calibration technique outlined above. But it was also found during the course of the running that the pedestal level varied with the beam intensity. Electrons from ionized argon atoms were collected in approximately $400 \mathrm{nsec}$. (See Appendix A) The positively charged argon atoms themselves had a mobility $10^{3}-10^{4}$ times lower than the electrons and their drift time thus was on the 
order of milliseconds. The positive ion drift in the liquid argon induced a small DC current in the amplifiers which resulted in a pedestal shift proportional to the current. The magnitude of the pedestal shift was determined as a funtion of intensity and corrections were applied. The effect was worst nearest the beam region since the "activity" there was the highest. At the maximum intensity used in the data taking, the positive ion drift resulted in a pedestal increase, in the strips nearest the beam, of approximately 300 MeV.

The average gain for each amplifier was also measured in every run. The gains were tracked to see if any appreciable change took place over the course of the 2 month data taking period. As the fluctuations were less than 28 on the average, no attempts were made to correct the gains for drifting. Although the electronic gains remained stable, the observed pulse heights changed substantially during the run, probably due to an improvement in the charge collection efficiency. There will be more discussion of this point in Section $C$.

Once the pedestal of a channel was subtracted, it was necessary to convert the remaining ADC counts to an energy. Conversion factors from counts to $\mathrm{GeV}$, the second type of constant mentioned above, were determined for every 
amplifier. Overall, the amount of charge collected depended on (see Appendix A) the high voltage on the gap and the purity of the argon. Conversion factors for each channel, in addition, depended on the capacitance of that channel, the amplifier gain and the amount of argon in the detector gaps corresponding to that channel.

These conversion factors were determined with electrons in a calibration run using a negative $50 \mathrm{GeV}$ beam. The electrons were identified with the $\mathrm{RI}$ Cerenkov counter, and were momentum analyzed in the spectrometer. The energies as determined in the spectrometer were compared to the pulse heights read out from the LAC. From this comparison the conversion factors were obtained. The detector was scanned with the electron beam, both in $\mathrm{x}$ and in $Y$. The $X$ scans, for the different $Y$ coordinates were obtained by moving the detector on airpads along the $x$ direction. The $Y$ scans, for two different $X$ coordinates in the left and right portion of the detector, were made possible by sweeping the beam vertically using the dipole magnet ElPM. (See Figure II.)

The following procedure was used for determining the conversion factors. First, cuts were applied to eliminate pions misidentified as electrons, and electrons that were accompanied by Bremsstrahlung photons. These cuts eliminated 
about 168 of the data. Conversion factors for the $x$ strips were determined from the $X$ scans and similarly for the $Y$ strips. The data in each scan was used to minimize the quantity $\sum_{1}\left(E_{i}{ }^{O}-E_{i}\right)^{2}$ where the summation was over all events. $E_{i}{ }^{\circ}$ was the reference energy for each event and was defined by $P_{i} / 2-E_{i}$ back. $P_{i}$ was the momentum of the electron as determined by the drift chambers and $\mathrm{E}_{i}$ back was the energy deposited in the back half of the LAC. The determination of $\mathrm{E}_{\mathrm{i}}$ back will be discussed later. The energy measured by the LAC was defined as $E_{i}=\sum_{j} a_{j} n_{i} j$ where $a_{j}$ was the conversion factor for each channel and $n_{i} j$ the number of ADC counts in that channel. The five strips around the shower maximum were used in the summation above. Individual calibration of the strips in the back half of the detector was somewhat difficult since the typical energy deposit, for an electron, was only about 138 of the total. Instead, a constant factor (13 MeV/count) was assigned to all of the channels in the back (except near the beam region). The value of $13 \mathrm{Mev} / \mathrm{count}$ for the constant factor was determined by minimizing the energy resolution.

The value of $13 \mathrm{MeV} /$ count was used initially for all of the front channels also. Subsequent iterations of the minimizations replaced this value with individual conversion factors for each strip. The final values for the conversion factors are shown in Figure 23. The conversion factors for 

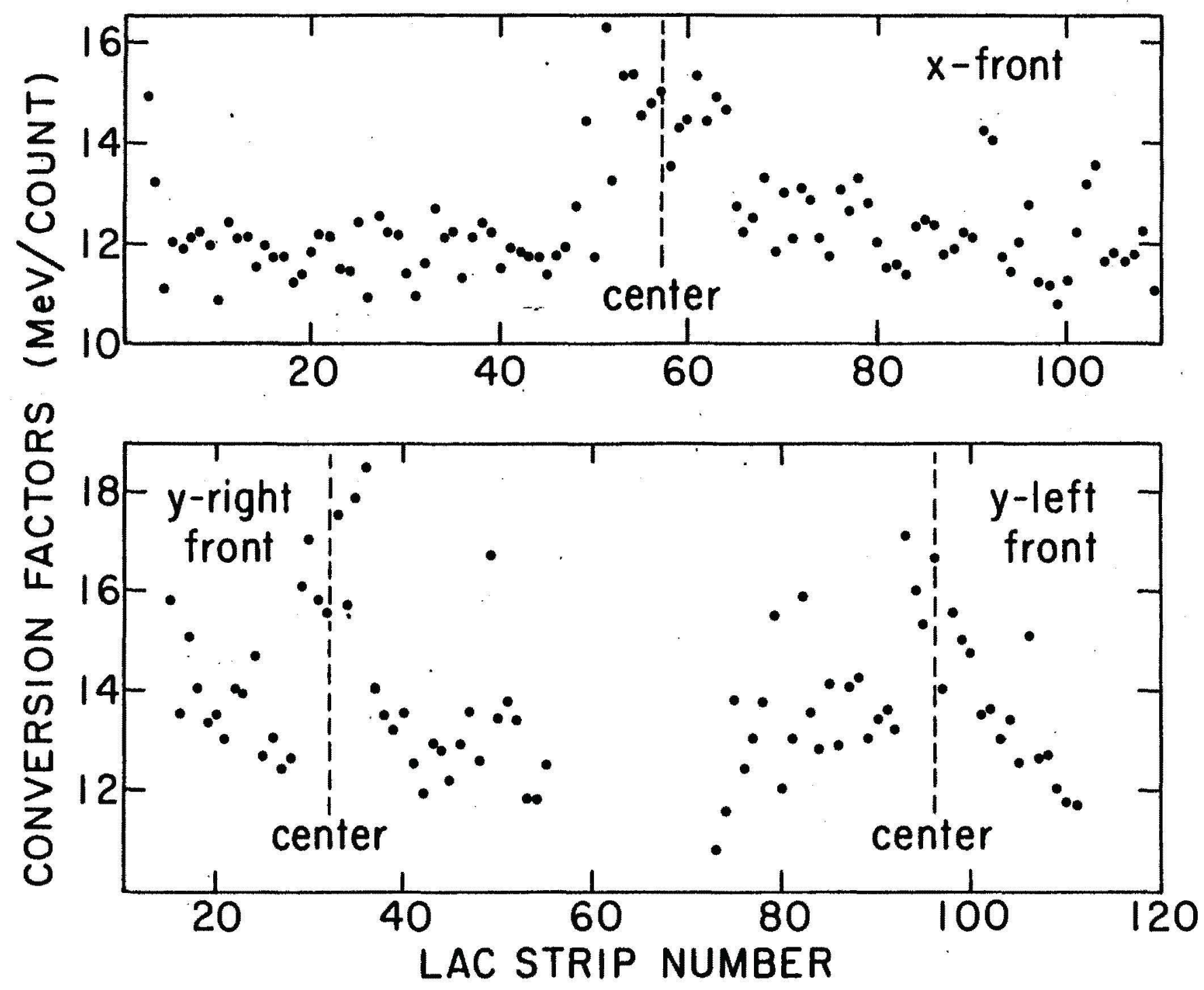
the strips near the beam were about 208 higher than the rest. As mentioned in Chapter II, the RC decay time for the amplifiers in this region was decreased to prevent possible saturation. This change also had the effect of decreasing the amplifier gain.

Using the final conversion factors, a resolution $(\sigma / \sqrt{ } \mathrm{E})$ of between $10 \%$ and $12 \%$ was obtained for the LAC. The $10 \%$ figure derived from the $Y$ scans, while the $12 \%$ figure was from the $x$ scans. The deterioration of the resolution observed in the $x$ scans might have resulted from some remaining contamination from events containing Bremsstrahlung photons. The ratio of the electron energy determined by the LAC to the electron energy determined by the spectrometer is shown in Figure 24. The curve through the data is a Gaussian whose rms width ( $\triangle E / E)$ equals 1.468. The smaller Gaussian shows the spectrometer(i.e. drift chamber) contribution to the energy resolution. Figure 25 shows the position resolution obtained with electrons in the calibration run. Again, the narrow Gaussian shows the contribution to the resolution expected from the spectrometer. The Gaussian curve drawn through the data has an rms width of $0.67 \mathrm{~mm}$. Unfolding the spectrometer resolution results in a spatial resolution of $0.58 \mathrm{~mm}$ for the LAC. 

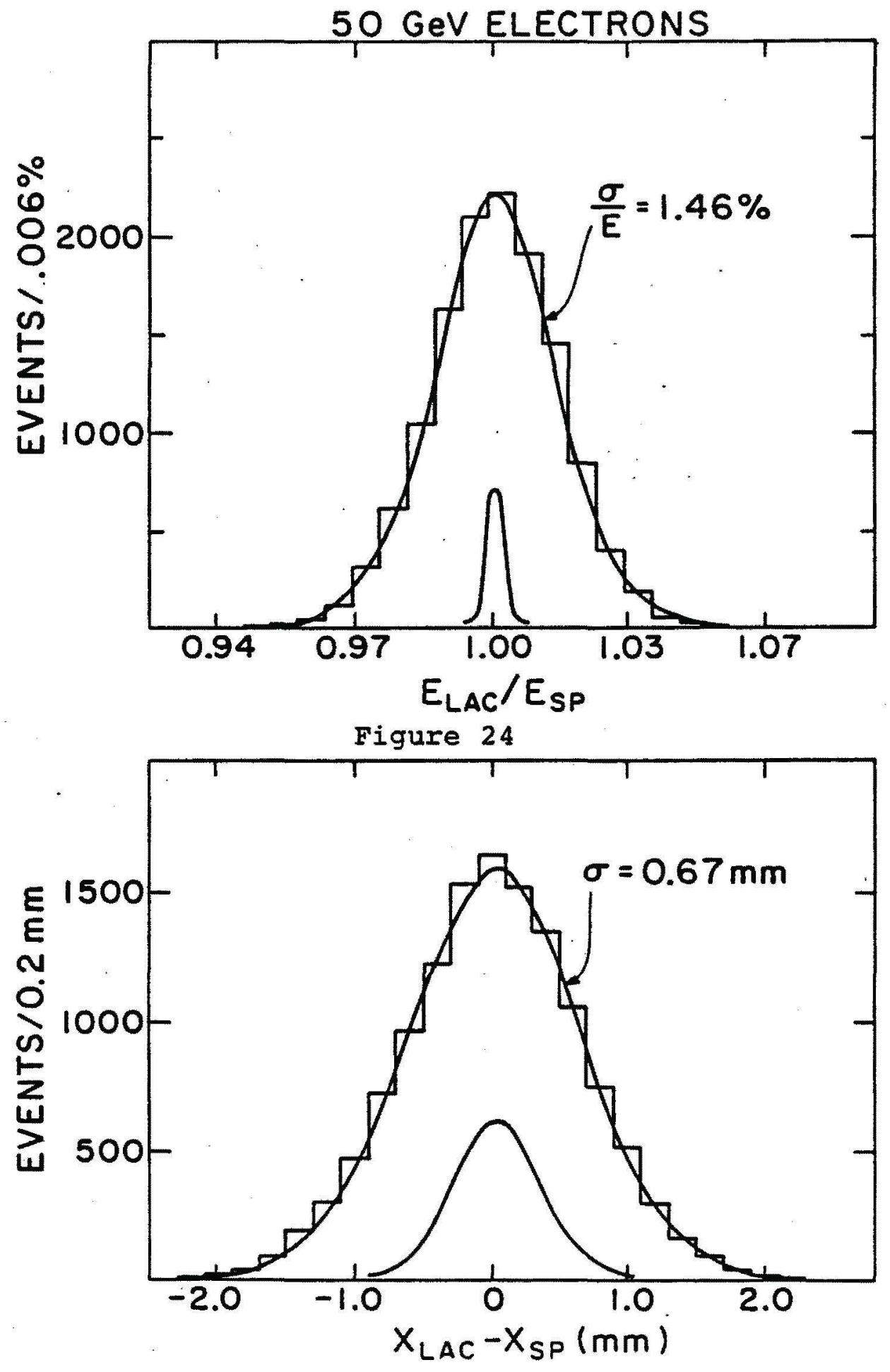

Figure 25 
The resulition figures obtained in the calibration run were somewhat better than those seen in the actual data taking. The operating conditions in the calibration run were more "ideal" than the conditions present during normal data taking. A more realistic idea of the operating resolution of the detector was provided by a sample of $\mathrm{K}^{+}+\mathrm{e}^{+} \pi^{\circ} \mathrm{v}_{\mathrm{e}}$ and $\mathrm{K}^{+} \rightarrow \pi^{+} \pi^{\circ}$ decay events taken with the regular trigger. The $\mathrm{K}^{+}+\mathrm{e}^{+} \pi^{\circ} \mathrm{v}_{\mathrm{e}}$, or $\mathrm{Ke} 3$ events, provided a spectrum of positron energies from $20 \mathrm{GeV}$ to $80 \mathrm{GeV}$. Figure 26 shows the positron energy determined by the LAC plotted versus the positron energy determined by the spectrometer. The response is roughly linear up to $80 \mathrm{GeV}$. In Figure 27 , the square of the rms energy resolution of the LAC for positrons $\left(\sigma^{2}=\left(E_{L A C}{ }^{-E_{S P E C}}\right)_{\text {rms }}{ }^{2}\right)$ is plotted versus positron energy. The dashed line through the data is given by

$$
\sigma^{2}=(0.14 \sqrt{\mathrm{E}}(\mathrm{GeV}))^{2}+(0.55)^{2}
$$

The $\sqrt{E}$ term represents the contribution to the resolution from sampling fluctuations while the constant terms represents pedestal shifts, amplifier noise, and related effects. The electron calibration point is shown for comparison. Note that the resolution for the ke 3 events is about a factor of 1.5 worse than the resolution for the electron calibration events. The discrepancy can be attributed to a number of factors: 

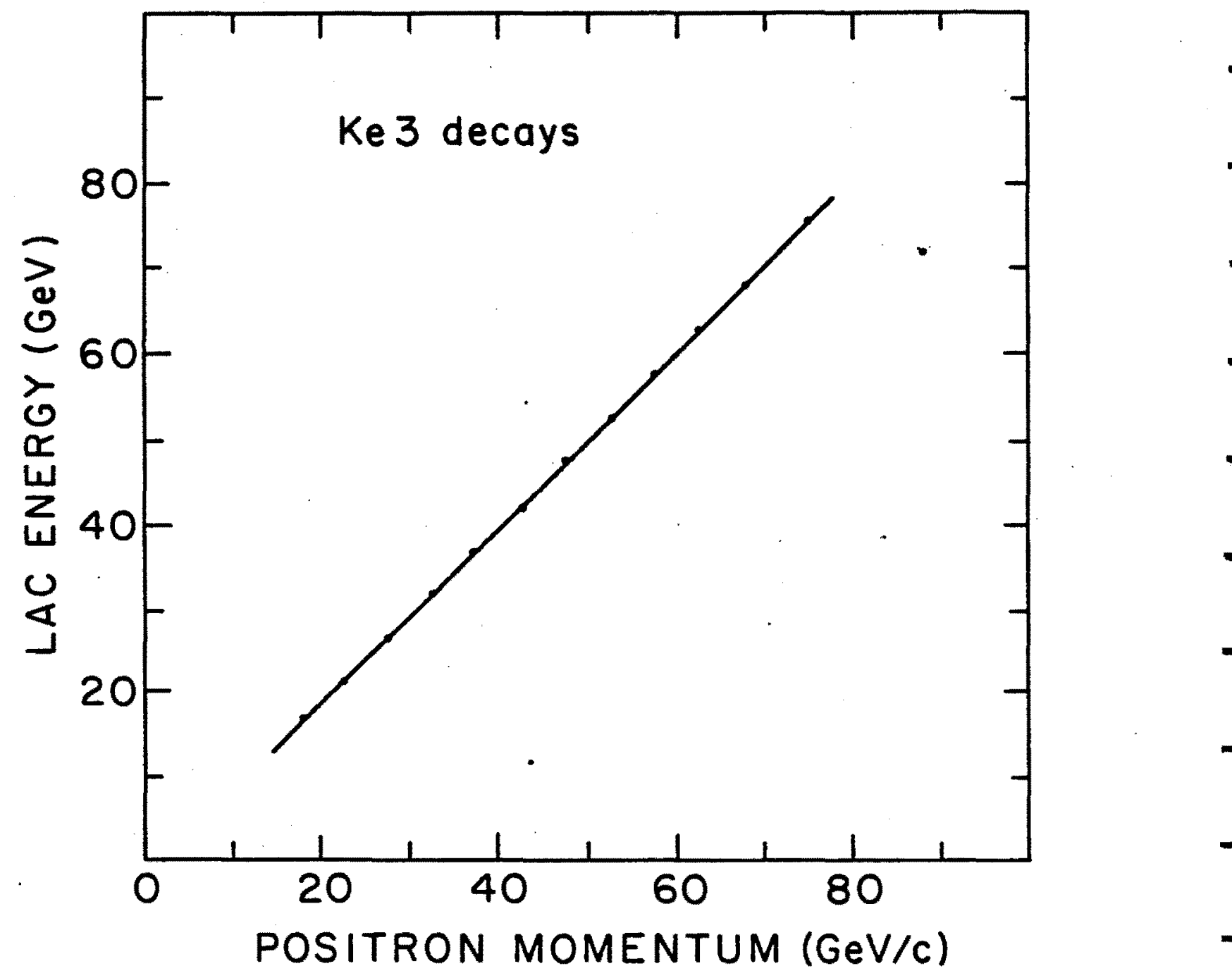

Figure 26 
$\mathrm{Ke}_{3}$ DECAYS

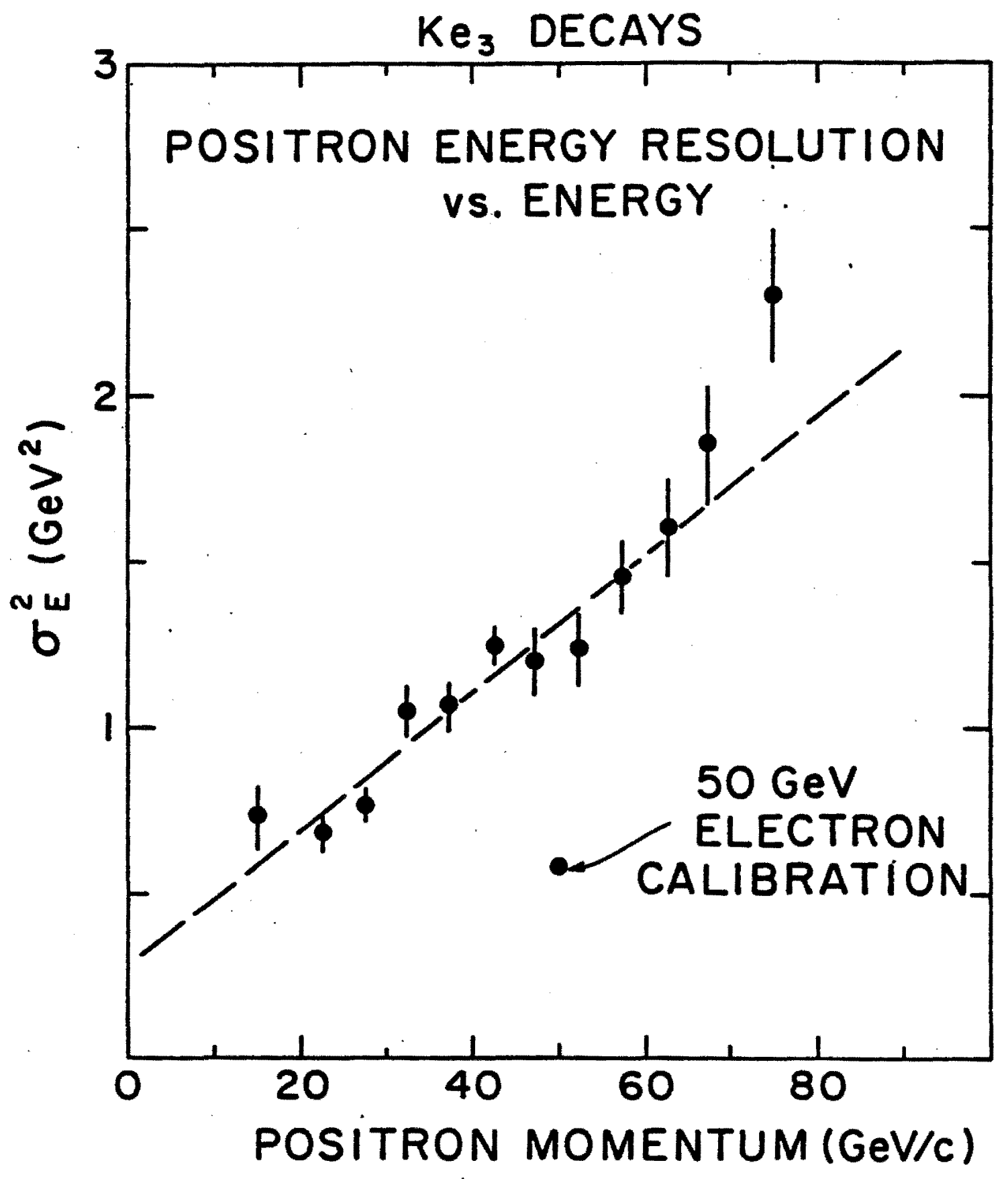

Figure 27 
1. The Ke3 events were accumulated over a period of several months. The calibration events were taken in one day. Any drifts or changes of LAC parameters during the calibration period would be insignificant.

2. The beam intensity during calibration was kept very low. During data taking, the intensity varied up to $5 \times 10^{5} / \mathrm{sec}$. Structure in the beam rate could have produced sudden pedestal shifts in the amplifiers.

3. The positrons from the Re3 events were spread over the detector in $X$ and $Y$. In the calibration the electrons had a constant $Y$ position in the $X$ scanning and vice versa.

4. The two photons from the $\pi^{\circ}$ could sometimes overlap in one view with the shower from the positron; the resulting confusion could have degraded the resolution. In the calibration, only one particle was incident on the detector at a time.

5. The positrons from $\operatorname{Re} 3$ decays came in at a multitude of angles, while the calibration electrons came in essentially at a single angle. 
The study of the energy resolution obtained using Re3 decays was informative because it demonstrated that resolution values obtained in calibration runs cannot be indiscriminately applied to data taking situations.

The position resolution of the LAC obtained for positrons from Re 3 decays is shown in Figure 28. The resolution obtained for $50 \mathrm{Gev}$ calibration electrons is shown also. The vertical scale is the rms position resolution $\left(\sigma_{\text {LAC }}=\left(x_{\text {LAC }}{ }^{-x_{\text {SPEC }}}\right)_{r m s}\right)$. The feature most noticeable is the rapid degradation of position resolution at low positron momentum. At low momentum the positrons are bent at large angles into the LAC. At large entrance angles, fluctuations in the longitudinal shower development can lead to significant errors in the measurement of the transverse position of the shower. This degradation in the position resolution at large angles was not important for the main body of the $\mathrm{K}^{+} \rightarrow \pi^{+} \pi^{\circ}$ and $\rho^{+}+\pi^{+} \pi^{\circ}$ data, since the entrance angles were very small in these cases.

The electromagnetic and hadronic shower shapes, as determined from calibration electrons and $\pi^{+}$'s from $\mathrm{K}^{+} \rightarrow \pi^{+} \pi^{\circ}$ decays respectively, are shown in Figure 29. studies of photon showers at different energies showed that the electromagnetic shower shape was independent of energy at the 58 leve1. [44] The shower shape was used in the photon 


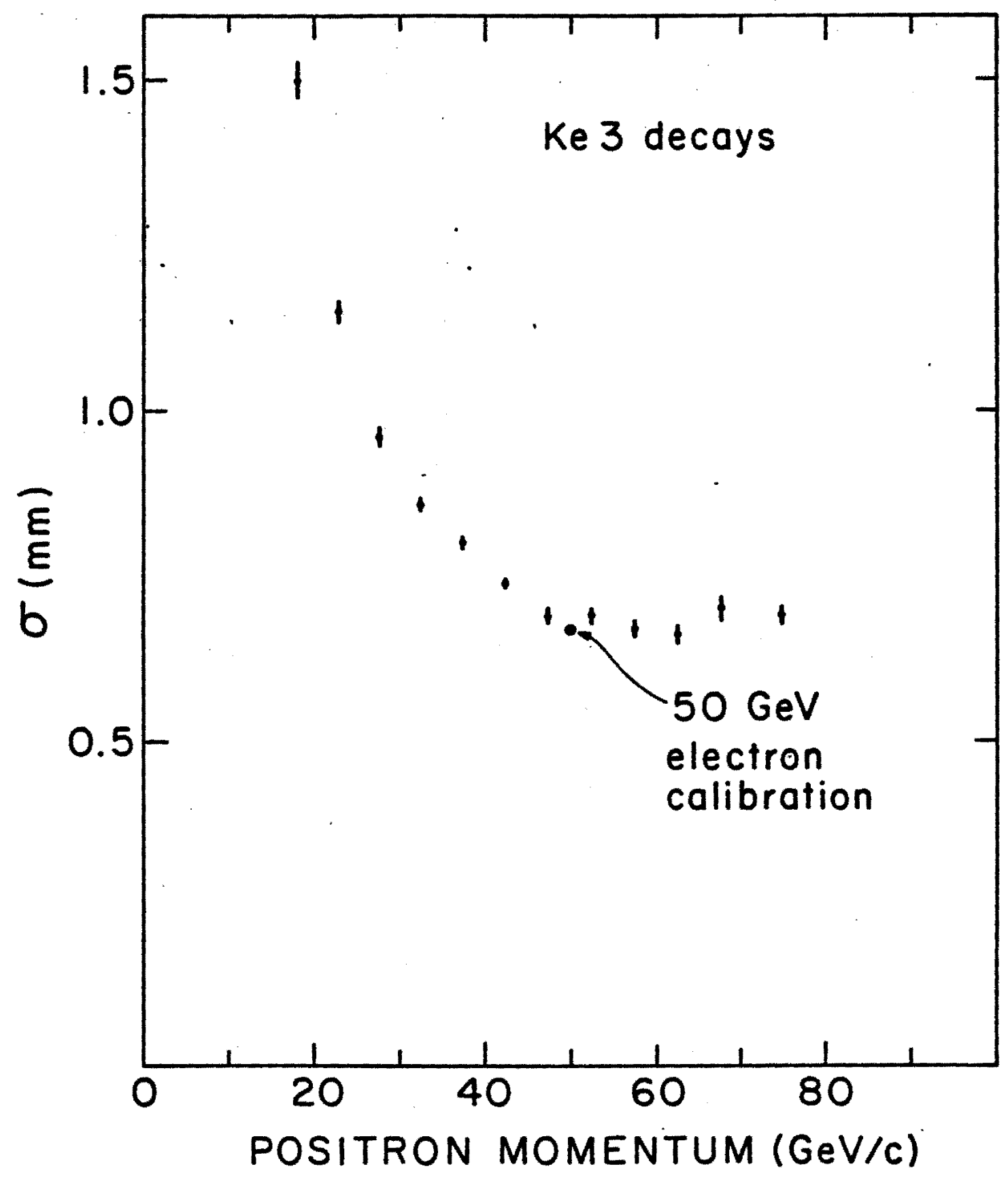

Figure 28 


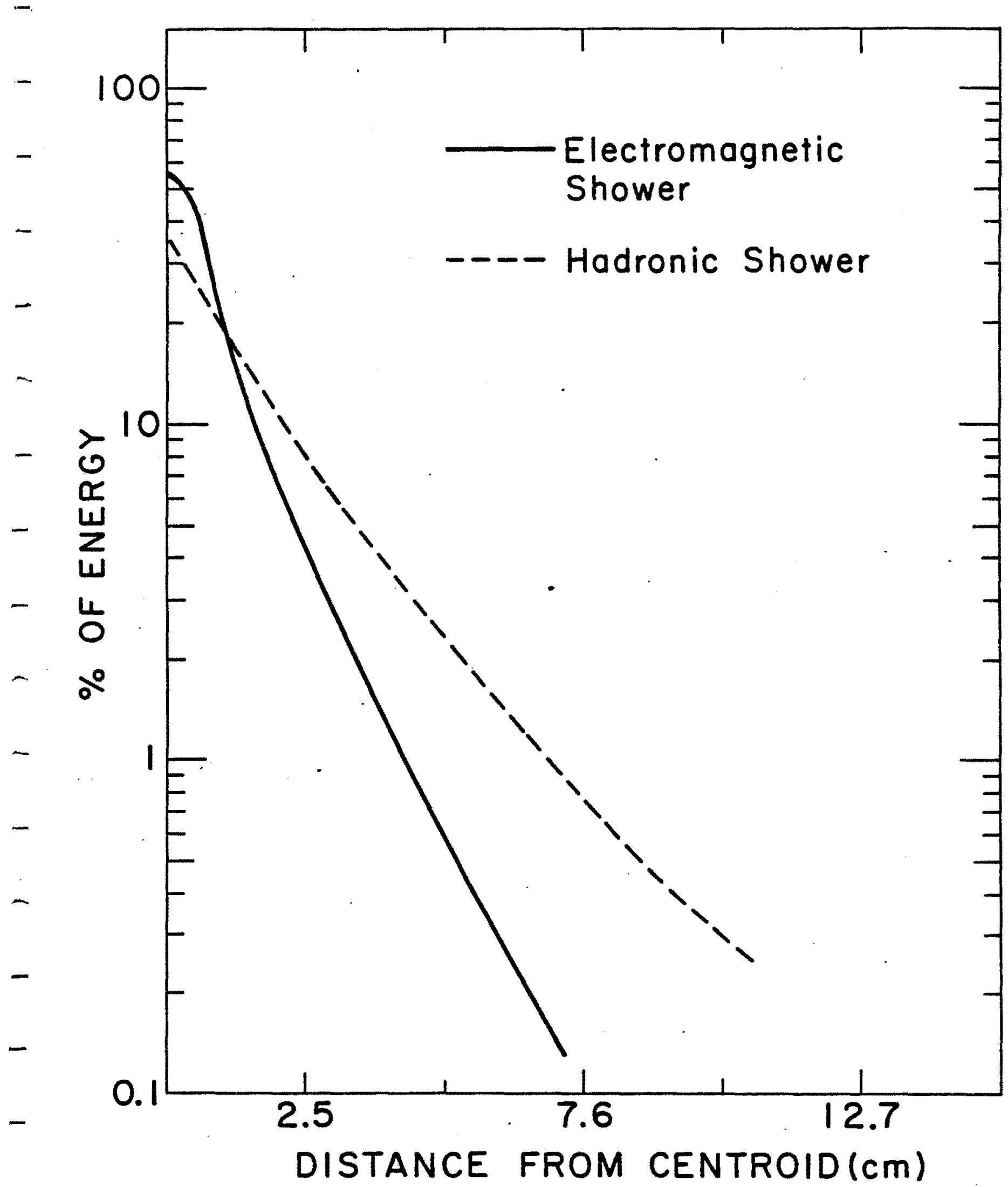

Figure 29 
reconstruction program to separate photons with partial overlaps in one view.

$$
\pi^{-'} \text { 's from } \mathrm{K}^{+} \rightarrow \pi^{+} \pi^{+} \pi^{-} \text {decays (taken with another }
$$
experimental trigger) were used to study the response of the LAC to hadrons. This decay was particularly useful for studying hadron response because no photons were involved and the $\pi^{-}$was bent into the opposite side of the detector from the two $\pi^{+\prime}$ s. It was determined that approximately $36 \%$ of all $\pi^{-1}$ s did not interact in the LAC. This is another way of saying that that the LAC presented one absorption length to the pions. The relative energy distribution( energy deposited in LAC/ total energy) for all pions that deposited more than $5 \mathrm{GeV}$ in the LAC is shown in Figure 30a. The distribution of EBACR/ETOT for both electrons and pions is shown in Figure 30b. Their different characteristics in this distribution enabled photons to be discriminated from hadrons.

It was important that the position of the LAC be established to a high accuracy in order for the $t$ (or square of the momentum transfer) of Reaction (1.1) to be calculated correctly. Two methods were used in the position measurement with each giving a similar answer. 

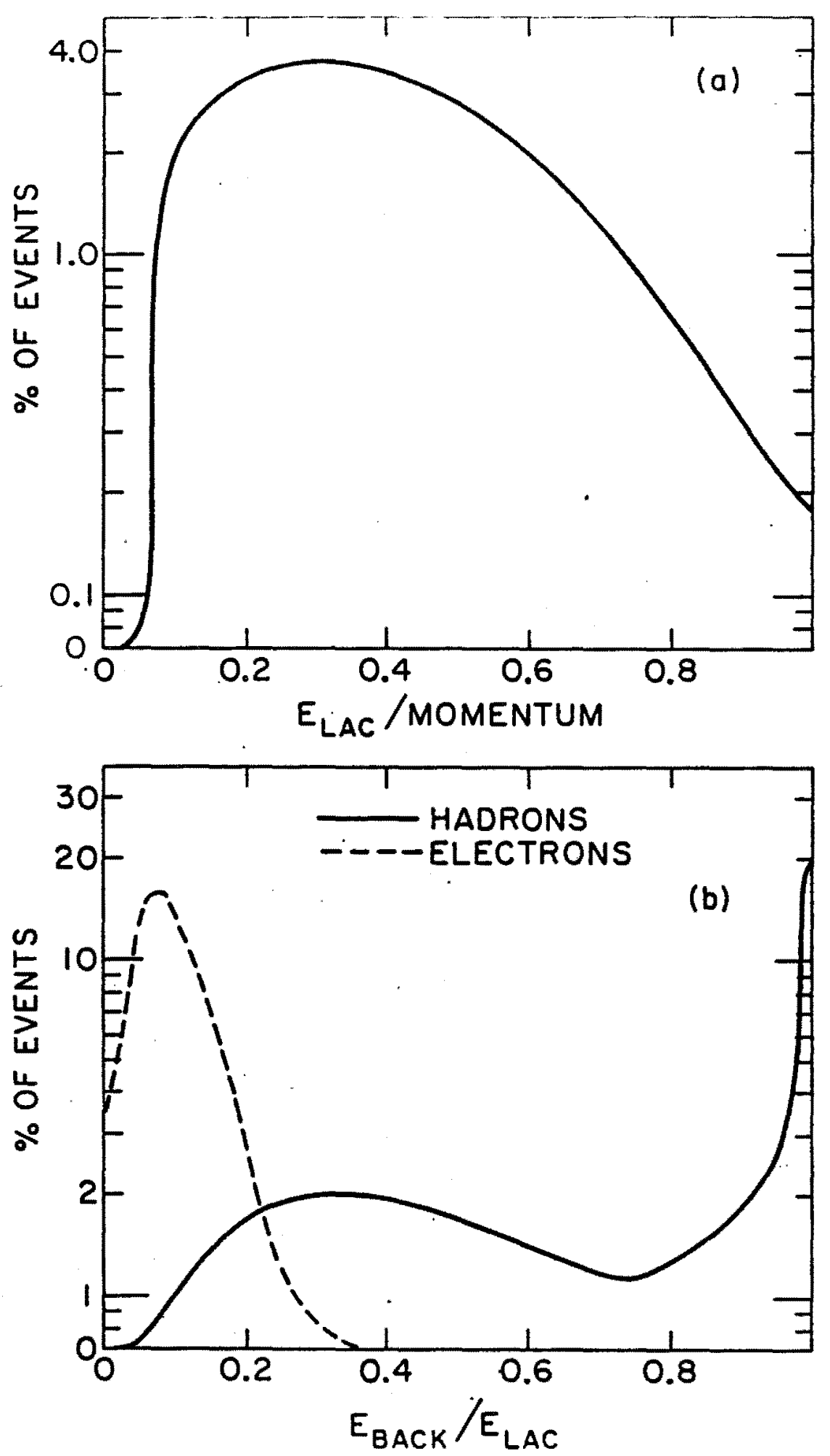

Figure 30 
The first method made use of the Re3 events collected in the run, along with a sample of pion electron scattering events collected with another experimental trigger. Showers were deposited in different sides of the LAC in the two types of events, since one involved electrons while the other involved positrons. This was very helpful in that a comparison of the position measurements tended to eliminate systematic effects. In this method the shower position(in each coordinate) determined by the photon reconstruction program was compared to the projection, at the $z$ position of the LAC, of the electron(positxon) trajectory determined by the drift chamber system. The LAC position in $X$ and $Y$ was shifted to obtain the best agreement. It should be mentioned that this method was sensitive enough to detect $a 0.5$ milliradian tilt( $X-Y$ plane) to the LAC.

The second method was somewhat more heuristic but perhaps more germane to the physics. Use was made of the thousands of $\mathrm{K}^{+} \rightarrow \pi^{+} \pi^{\circ}$ events collected in the Rho trigger. Since the process $\mathrm{K}^{+}+\pi^{+} \pi^{\circ}$ is a decay, intrinsically it takes place with a $t$ of zero. A measurement of the actual distribution in $t$ of these decays gives an idea of the resolution of the spectrometer. If the spectrometer has Gaussian resolution, the distribution in $t$ of the $R$ decays should look like $e^{-t / 2 \sigma^{2}}$, where $\sigma$ is the resolution in $p$. (See Appendix B for more details.) The second method 
involved shifting the LAC position in $x$ and $y$ until the resolution in $t$ or $p_{t}$ observed was a minimum. In Figures $31 a$ and $31 \mathrm{~b}$ is shown the resolution observed as a function of LAC position (position shifts are measured with respect to the LAC positions obtained using the first method). A clear minimum is seen for a LAC position shifted in $X$ by $30 \mathrm{mils}$ from that determined by the first method, and in $Y$ by 8 mils. For most of the analysis, the LAC position obtained by the second method was used. Physics results were checked with the position obtained by the first method, however, and were found to be consistent. (See Chapter VI.)

B. Reconstruction

The "hits" and pulse height information recorded on magnetic tape were processed through a reconstruction program to determine the positions and energies of the particles in the events. The reconstruction process is described below.

\section{B.1 Charged Track Reconstruction}

The first step in the charged track reconstruction program was the conversion of the TDC counts to spatial positions in the drift chambers. This was done using the calibration constants described in section $A$. Since there was no way to distinguish between the left and right side of 


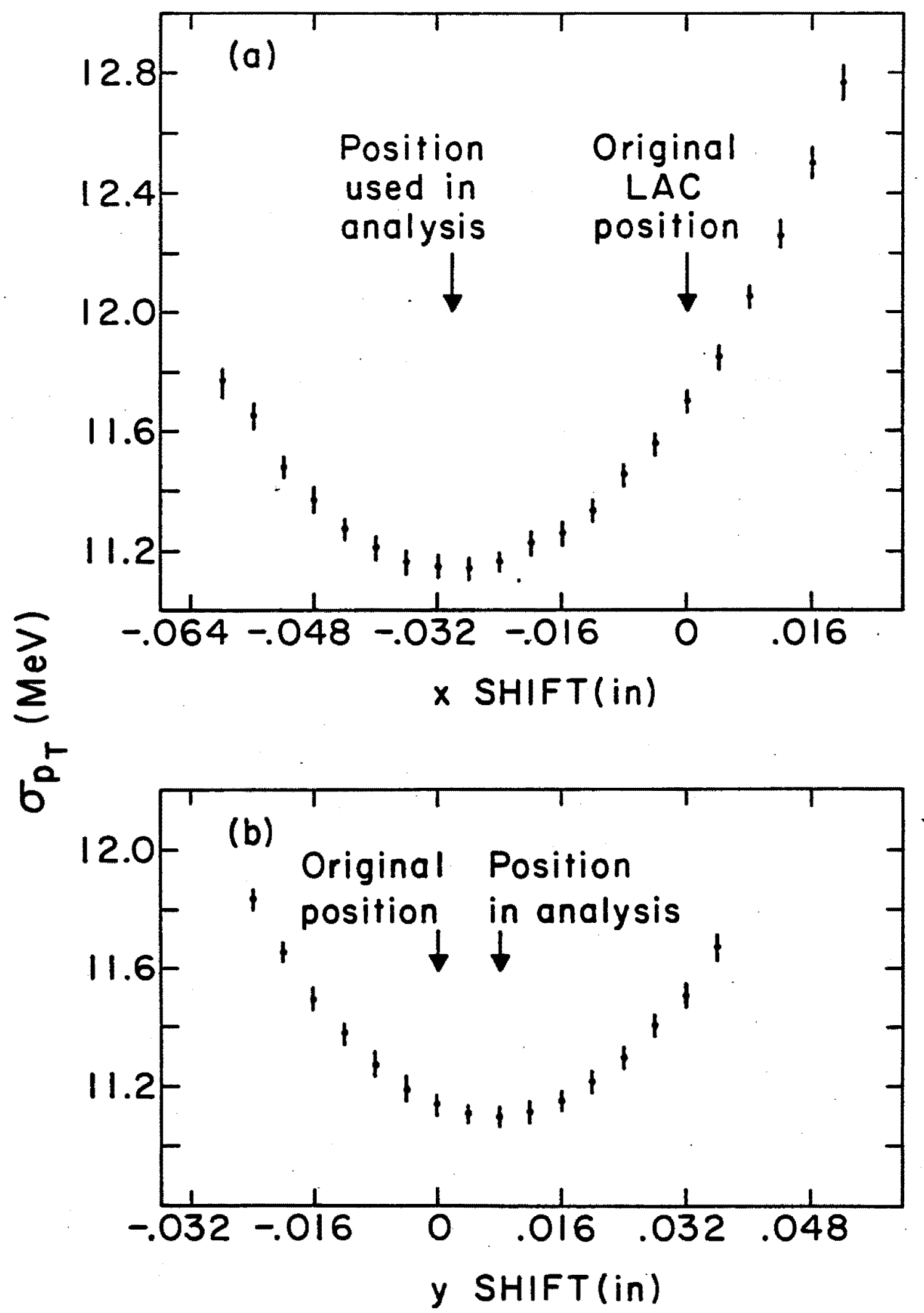

Figure 31 
a wire in a single cell, two spatial coordinate candidates were generated for every "hit" wire.

Downstreap tracks were searched for using hits from the P2, D3, and D4 chambers. (See Figure 11 for the location of individual chambers.) It was easier to start with the downstream segments since multiple tracks would tend to be well separated due to the dispersion of the magnet. After finding hits in the $x$ and $U$ projections, hits in the $V$ projection were predicted and searched for. If a predicted hit was found, all of the downstream hits were fitted to a straight line. (A hit in a projection could come from either or both of the 2 staggered planes in that projection.) All possible combinations for which the hits were within $1.14 \mathrm{~mm}$ of the resultant straight line were considered and the fit with the best $\chi^{2}$ was chosen as the initial downstream track segment.

Once the downstream segments were defined, upstream track segments were searched for as follows. The BM109 magnet deflected charged particles only in the horizontal direction. Since the magnetic field of the BMI09 was very uniform, the trajectory of a charged particle passing through the magnet could thus be approximated by two straight line segments intersecting at the middle of the magnet and having the same $Y$ slope. This approximation provided the starting 
point for the search for the upstream track segment. Another point, and hence the upsteam $x$ slope, was provided by the hits found in the Pl chamber. Thus five parameters, the $\mathrm{X}$ and $Y$ coordinates of the track at the center of the magnet, the $x$ slopes of the upstream and downstream segments of the track, and the $Y$ slope common to both track segments, defined the trajectory at this point in the reconstruction process.

A weight $w_{i}=e^{-\left(r_{i}-r_{i}{ }^{0}\right) / 2 \sigma^{2}}$ was calculated for each hit within $3.8 \mathrm{~mm}$ of the track hypothesis. In the weight, $\left(r_{i}-r_{i}{ }^{\circ}\right)$ was the distance between the hit and the track and $\sigma$ provided a measure of the uncertainty in the fitting. Initially $\sigma$ was set equal to $6.4 \mathrm{~mm}$. An iterative fitting procedure was carried out, with the value of $\sigma$ being reduced by a factor of $3 / 4$ in each step. Also, in each step, a new set of track parameters was generated and the hits with low weights were discarded. The left/right ambiguity for each wire was solved by discarding the hit in the pair with the lower weight. In the final fit to define the track, the search radius was reduced from $3.8 \mathrm{~mm}$ to $1 \mathrm{~mm}$.

The beam track was defined using the hits in the $\mathrm{Jl}$ and $\mathrm{J} 2$ chambers. The interaction vertex was defined to be the point of closest approach of the beam track and the upstream track segment. Using $\mathrm{K}^{+} \rightarrow \pi^{+} \pi^{\circ}$ decays it was found that the $\mathrm{K}^{+}$beam track could be linked with an outgoing track 
over 95 of the time. About 18 of the loss was due to chamber inefficiency, while the remainder resulted from the strictness of the program's track finding criteria.

\section{B.2 Photon Reconstruction}

Photon positions and energies were calculated from the LAC information by the photon reconstruction program(GAMREC). For every event, the strips with energies exceeding $100 \mathrm{MeV}$ were digitized, and the pulse height information was written onto magnetic tape. The first step in the reconstruction process was to subtract the appropriate pedestal level for each channel(determined on a run-by-run basis) and to convert the remaining digital counts into energy using the conversion factors determined in the calibration procedure.

The next stage involved pattern recognition. The two views, $X$ and $Y$, were treated separately at first. Groups of strips containing significant energy were searched for in both views. Each group was divided into individual peaks, with two peaks being considered distinct if at least one strip between them had a smaller pulse height. The energy of each peak was determined by subtracting the tails of neighboring peaks. The tails were generated by using shower shape information from the calibration run. This procedure 
was iterated until energies of all the peaks were stable. Each individual peak was called a "gamma".

Positions of gammas were determined using a 3 strip algorithm. With $P 2$ being the pulse height in the peak strip, Pl the pulse height in the strip to the left and P3 the pulse height in the strip to the right, the position, in units of strip width, was determined using the following algorithm

$$
x-x_{0}= \pm\left[0.4+A\left(C l \mp V\left(C 2+C 3 / A^{2}\right)\right)\right]
$$

with $A=(P 3-P 1) / P 2-C 4$ and $X_{0}$ being the center of the peak channel strip. The constants $\mathrm{Cl}, \mathrm{C2}, \mathrm{C} 3$, and $\mathrm{C4}$ were determined from calibration data. Note that because of the granularity of the detector strips, a simple energy weighted mean position algorithm would have led to errors in the position measurement.

Initially, the energy of each gamma was also determined using a 3 strip algorithm. In the later versions of the reconstruction program, a more sophisticated algorithm was employed that made use of up to 9 strips for the energy determination of each gamma. (The exact number of strips used depended on the energy of the individual gamma.) For both algorithms, corrections based on the known shower shape were made for the energy contained in the tails. 
After the energies and positions of all gammas in the detector were determined, gammas in the two views were correlated to form photons. The correlation was based solely on an energy matchup, since no $U$ or $V$ readout strips were employed in our detector. Since the $X$ and $Y$ readout boards were-interleaved, the LAC was in essence two semi-independent detectors, the resolution of each being approximately a factor of $\sqrt{2}$ worse than the combination. The high correlation of the pulse heights in the two views is shown in Figure 32, which is a scatterplot of the $X$ and $Y$ gamma energies for positrons from ke3 events.

For two gammas to be correlated the quantity

$$
\frac{\left|E_{X}-E_{Y}\right|}{\left[E_{X}+0.5+0.03 E_{X}{ }^{2}\right]^{1 / 2}}<0.5
$$

, with $E_{X}$ and $E_{Y}$ in GeV, was required to be less than a certain threshold value, which was increased in successive iterations of the search. Here, $\mathbf{E}_{\mathbf{X}}$ is the energy of the gamma in the $X$ view and $E_{Y}$ the energy of the gamma in the $Y$ view. After each successful correlation, the successfully correlated gammas were removed from further consideration.

Because of the high energies of the $\pi^{\circ}$ 's involved in this experiment, it was quite likely for the two photons of 


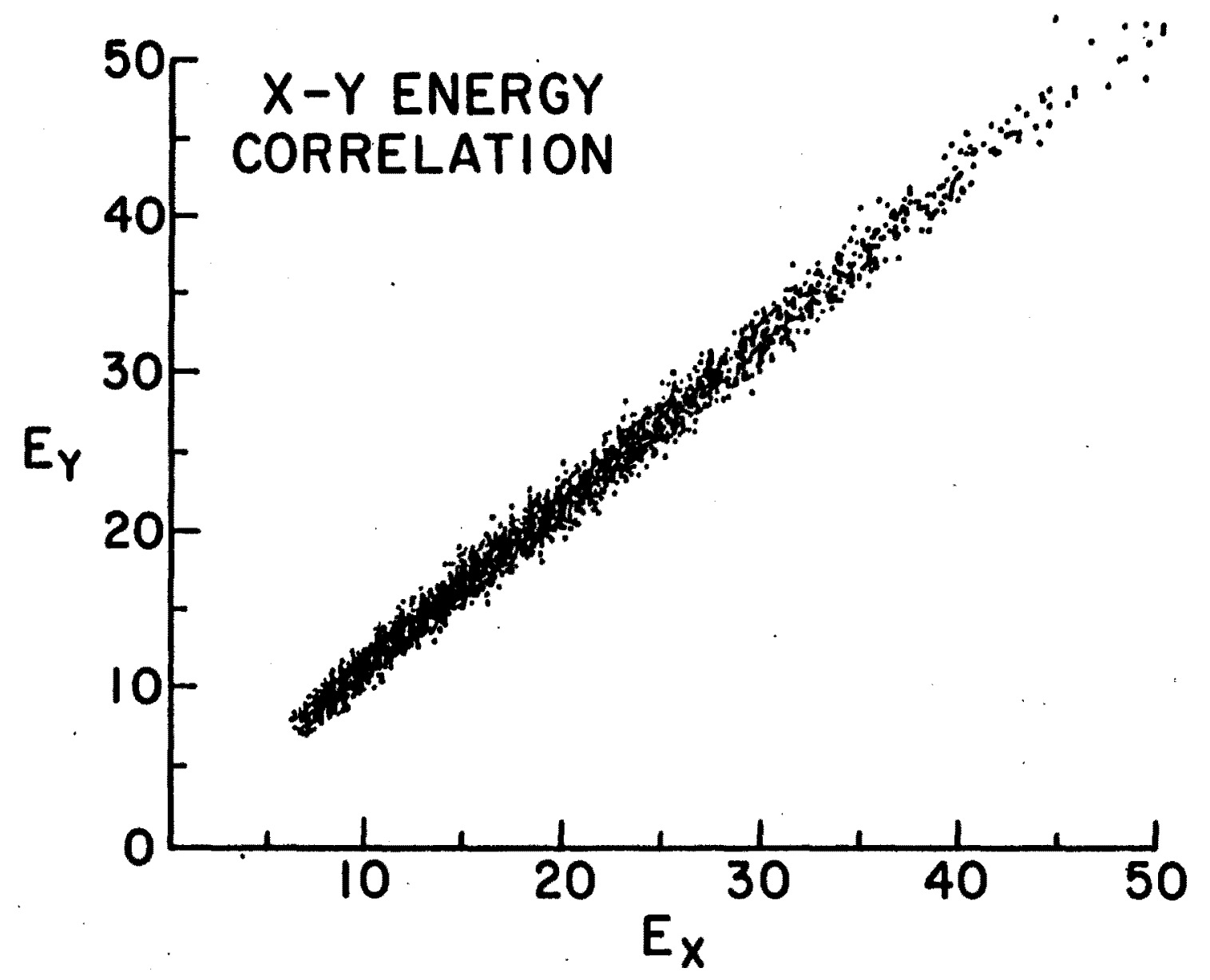

Figure 32 
the $\pi^{\circ}$ to appear merged in one view while appearing as resolvable gammas in the other view. After all isolated gammas were eliminated, the reconstruction program attempted to correlate the energy of two gammas in one view with a single gamma in the other view. If such a correlation was possible the positions of the two gammas in the merged view were redetermined with a fitting procedure that made use of the gamma energies found in the unmerged view.

The energy in the back half of the LAC was determined for each gamma with another 3 strip algorithm. The front and back were added to determine the total photon energy. The position of each photon was determined solely from the front half of the detector, as described before. Before being written onto summary tape, photon positions were compared to charged track positions. If a photon was within $2.5 \mathrm{~cm}$ of a charged track, the photon was labeled as a charged-photon overlap and flagged as an electron or hadron candidate.

C. Run by Run Consistency.

In the reconstruction process, the reconstructed energies, positions, and charged particle trajectories were written, for each data type, onto a summary tape. During the processing, various quantities in each data run were examined to ensure that the quality of data was consistent throughout 
the experiment. For example, chamber efficiencies and scaler rates were studied for each run to check for potential problems.

One good check of consistency was the measured decay rate for $\mathrm{K}^{+}+\pi^{+} \pi^{\circ}$ events. The number of these decays per data tape was sufficiently high that a \pm 10 o run by run comparison was possible. The runs that had a statistically low number of decays were left out of any further analysis. In this way seven data tapes, mostly from test runs, were rejected from further analysis.

The minimum bias beam triggers mentioned in . Chapter II were useful to check the alignment of the scintillation counters in the experiment. It was discovered that two of the counters, $B H$ and $s$, were misaligned during part of the running period. Correction factors, derived from the beam triggers, were applied to the data to correct for the misalignment (See Chapter V.)

In our experiment, the IAC was the apparatus most susceptible to variation over time. The $\pi^{\circ}$ mass (from $\mathrm{K}^{+} \rightarrow \pi^{+} \pi^{\circ}$ decays) was monitored from run to run to detect any change. As mentioned in section A.2, the gains of the LAC amplifiers were monitored and found to be constant(to within a few percent) over the course of the running period. The $\pi^{\circ}$ 
mass, however, was found to have increased by approximately 308 over this time period. The increase, therefore, must have been due to an improvement in charge collection. Figure 33 shows the relative collected charge/deduced from the observed $\pi^{\circ}$ mass) as a function of time(using selected runs). A roughly linear increase with time is seen. Since the high voltage on the LAC was monitored and found to remain constant, the increase in charge collection was interpreted as being due to the freezing out of some electronegative impurity in the liquid argon. A simple time dependent correction was applied to the conversion factors of the LAC amplifiers so that the $\pi^{\circ}$ mass, in each $r$ un, was normalized to the correct value. Appendix A discusses charge collection in the LAC in more detail. 


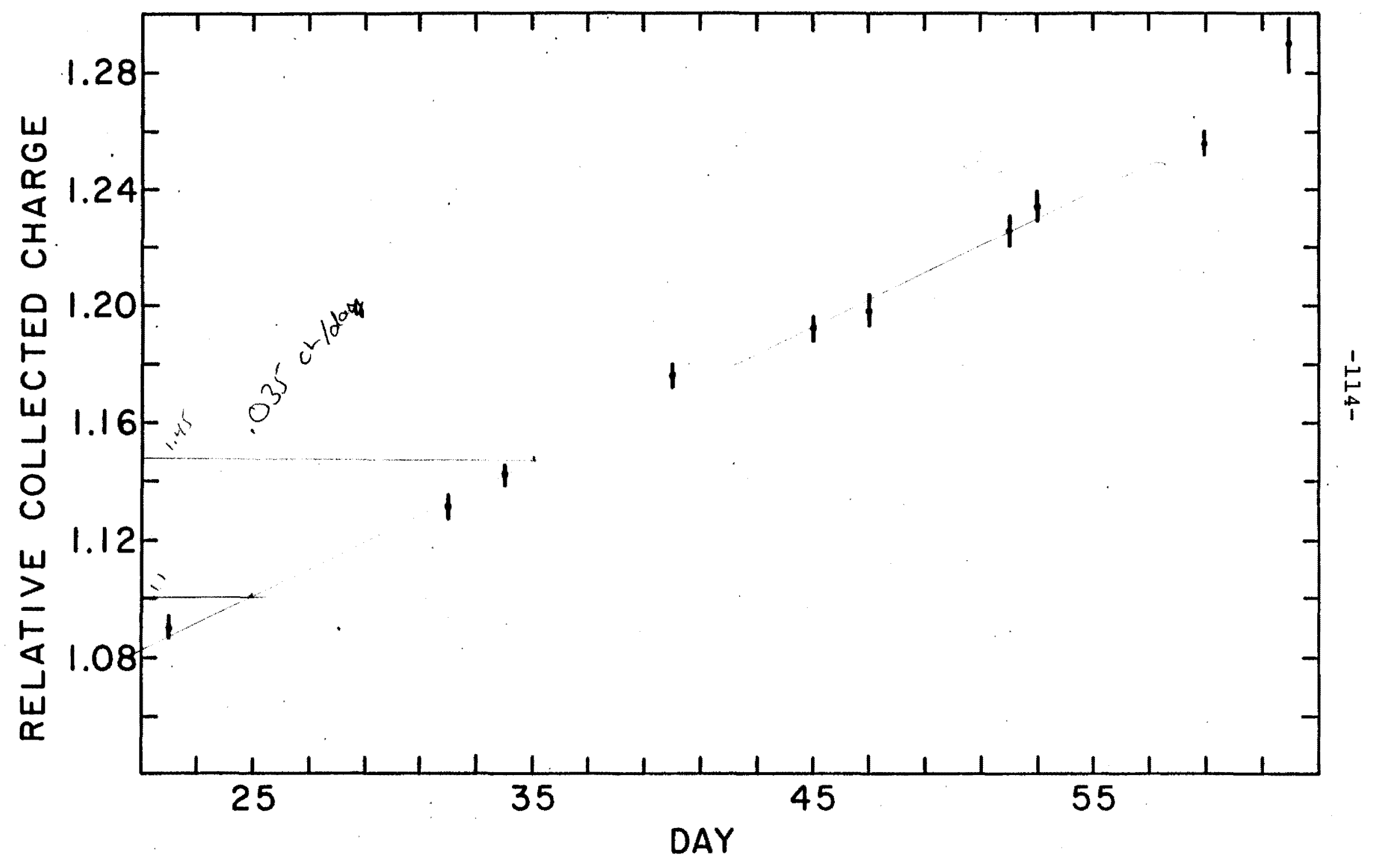

Figure 33 
Comparisons to Monte Carlo Model

The experiment was modeled in a Monte Carlo program so that the geometric and trigger acceptances of the spectrometer could be determined. By passing the Monte Carlo generated events through the same reconstruction program used for the experimental data, the efficiency of reconstruction was also determined. In this chapter the Monte Carlo model will be described. Also the resolution of the spectrometer will be discussed in more detail and comparisons will be made between Monte Carlo results and data.

A. Monte Carlo Model

Events of the type shown below

$$
\begin{aligned}
& \pi^{+}+(A, z) \rightarrow \rho^{+}+(A, z) \\
& k^{+}+\pi^{+} \pi^{+} \pi^{\circ} \\
& \mathrm{K}^{+}+\mathrm{e}^{+} \pi^{\circ} v_{\mathrm{e}}
\end{aligned}
$$


were generated with the Monte Carlo program and allowed to propagate through the spectrometer. The events were vetoed if any of the decay products left the geometrical acceptance of the spectrometer. For charged particles, hits were generated in the chambers along the particle trajectory and, for photons and electrons, electromagnetic showers were generated in the LAC. The various parameters necessary in the Monte Carlo program were determined using real events. The procedure used to determine these parameters is outlined below:

1. The angular divergence and transverse dimensions of the incident beam were determined from an analysis of bean tracks.

2. Positions of veto counters(BA, BV, VE, etc) were measured by looking for sharp cutoffs in charged particle distributions at the appropriate $z$ positions.

3. Efficiencies and noise levels of the chambers were determined from data. The beam region was desensitized to mimic the space charge effects seen in the experiment. 
4. The logic signals $T C$ and MBV were simulated using the generated hit distributions.

5. Multiple Coulomb scattering in the targets was simulated using a Gaussian approximation for the effect.

6. The transverse kick, $\int B \cdot d 1$, of the BMI09 magnet and its transverse dimensions were determined from studies of real data.

7. Photon and electron showers were generated in the LAC by distributing energy to the detector strips using the shower shape determined in the calibration. The energy deposited in each strip was smeared in a Gaussian fashion with $\sigma^{2}=(.14 \sqrt{ } \text { E) })^{2}+$ .003 . The first term corresponds to the energy dependent resolution observed in the LAC using Ke 3 decays. The second term is a constant term added to each strip to mimic the noise effects seen in real data. The total energy was divided between the front and back of the LAC according to the distributions found in the calibration studies.

8. Hadron interactions in the LAC were simulated using the characteristics discusssed in Chapter III. 
9. The LAC signals EUP, EDOWN, and ETOTAL were calculated with the strip pulse heights in the same manner as in the actual experiment. The Monte Carlo events were required to satisfy the LAC logic simulation.

The events generated in this manner were then passed to the reconstruction program discussed in Chapter III. The degree to which the Monte Carlo successfully mimics the experiment will be shown by the agreement between experimental and Monte Carlo distributions.

B. Comparison of $\mathrm{R}^{+} \rightarrow \pi^{+} \pi^{\circ}$ decays-Monte Carlo and Experiment

The experimental trigger was sensitive to $\mathrm{K}^{+}+\pi^{+} \pi^{\circ}$ decays which occurred in the interval from approximately $1 \mathrm{~m}$ upstream of the target to $6 \mathrm{~m}$ downstream. The experimental data set contained a much larger sample of $\mathrm{K}^{+}$decays than of $\rho^{+}$events. Topologically, the two types of events were very similar. This topological similarity was taken advantage of by using the normalization(number of decays expected/number of decays observed) of the $\mathrm{K}^{+}+\pi^{+} \pi^{\circ}$ decay rate as a correction factor to the $\rho^{+}$cross section. This point will be discussed in more detail in the next chapter. The data sample of $\mathrm{K}^{+} \rightarrow \pi^{+} \pi^{\circ}$ decays was large enough to allow a statistically significant comparison of the experimental distributions to 
the Monte Carlo generated distributions. As will be seen in the following discussion, there was a fairly good agreement between the data and Monte Car10, with the discrepancies not seriously affecting the analysis. The distributions below had restrictions, or "cuts", applied, whenever applicable, on the $z$ vertex of the decay, the 2 photon mass, the $\pi^{+} \pi^{\circ}$ mass, the total energy, and the reconstructed $t$. These cuts are described in more detail below and in section $\mathrm{C}$ of Chapter $\mathrm{v}$. Unless otherwise noted, the Monte Carlo prediction is indicated by a solid line.

In the Monte Carlo, kaons were allowed to decay uniformly in the region from $1.25 \mathrm{~m}$ upstream of the target to $6.25 \mathrm{~m}$ downstream of the target. A comparison, for $\mathrm{R}^{+} \rightarrow \pi^{+} \pi^{\circ}$ decays, between the experimental and Monte Carlo $\mathrm{z}$ vertex decay distributions is shown in Figure 34. The dip seen in the data near the target region is due to the presence of various veto counters. These counters were not included in the Monte Carlo model since the target region was avolded in any normalization procedure. The region that was used in the $\mathrm{K}^{+}$decay normalization is marked in the figure by the arrows.

Since kaons are spin 0 particles, the angular distribution of the charged pion from the $\mathrm{x}^{+}$decay, in the helicity frame, should be flat. (The Gottfried-Jackson and helicity frames are defined in Figures $4 c$ and $4 d$ for the case 


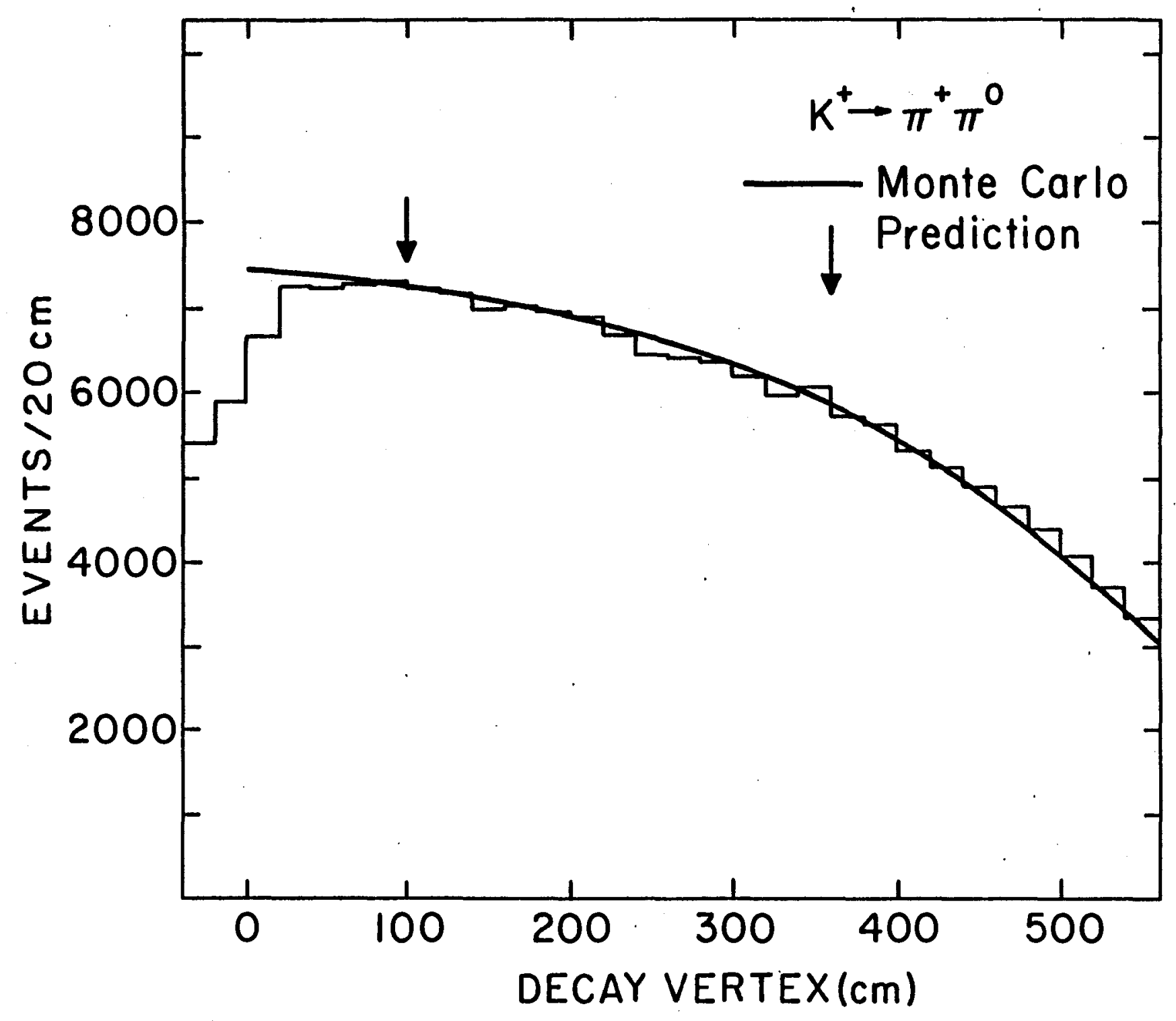


of $\rho^{+}$production.) A comparison of the Monte Carlo and experimental angular distributions is shown in Figure 35. The angle plotted here is the angle of the charged pion relative to the flight direction of the kaon, in the rest frame of the kaon. Both distributions are cut off at forward angles. Events with the charged pion at forward angles tended to be eliminated by the $M(B V)^{2}$, and the $B A$ and $V E$ counters. Also events of this type were lost due to vetoing of the low energy $\pi^{\circ} \cdot s$ in the $A M$ counters and due to the threshold imposed on energy deposition in the LAC. The good agreement indicates that the trigger and geometric acceptances have been modeled correctly in the Monte carlo.

As mentioned in the last chapter, the beam region in the drift chambers was deadened due to the buildup of space charge. In Figure 36 is shown a comparison between data and Monte Carlo of the transverse distance between the charged pion track and the beam axis. The $z$ position of the plot corresponds to the $\mathbf{P} 1 \mathrm{X}$ chamber. The agreement indicates that the chamber inefficiency did not cause any serious problem.

The asymmetry distribution for the two photons from the $\mathrm{R}^{+}$decay $\pi^{\circ} \cdot \mathbf{s}$ is shown in Figure 37. The asymmetry for the two photons is defined as 
$-122-$

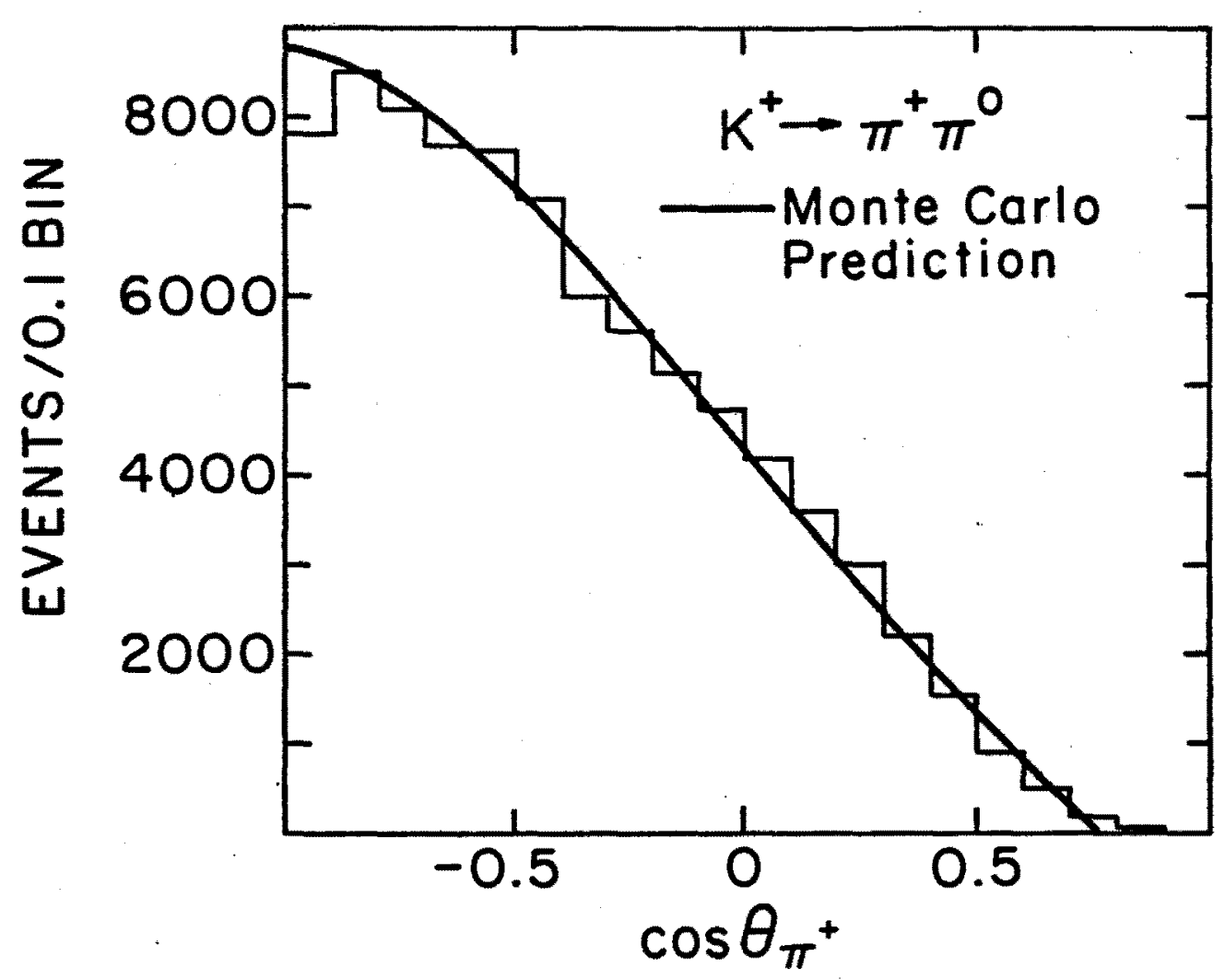

Figure 35

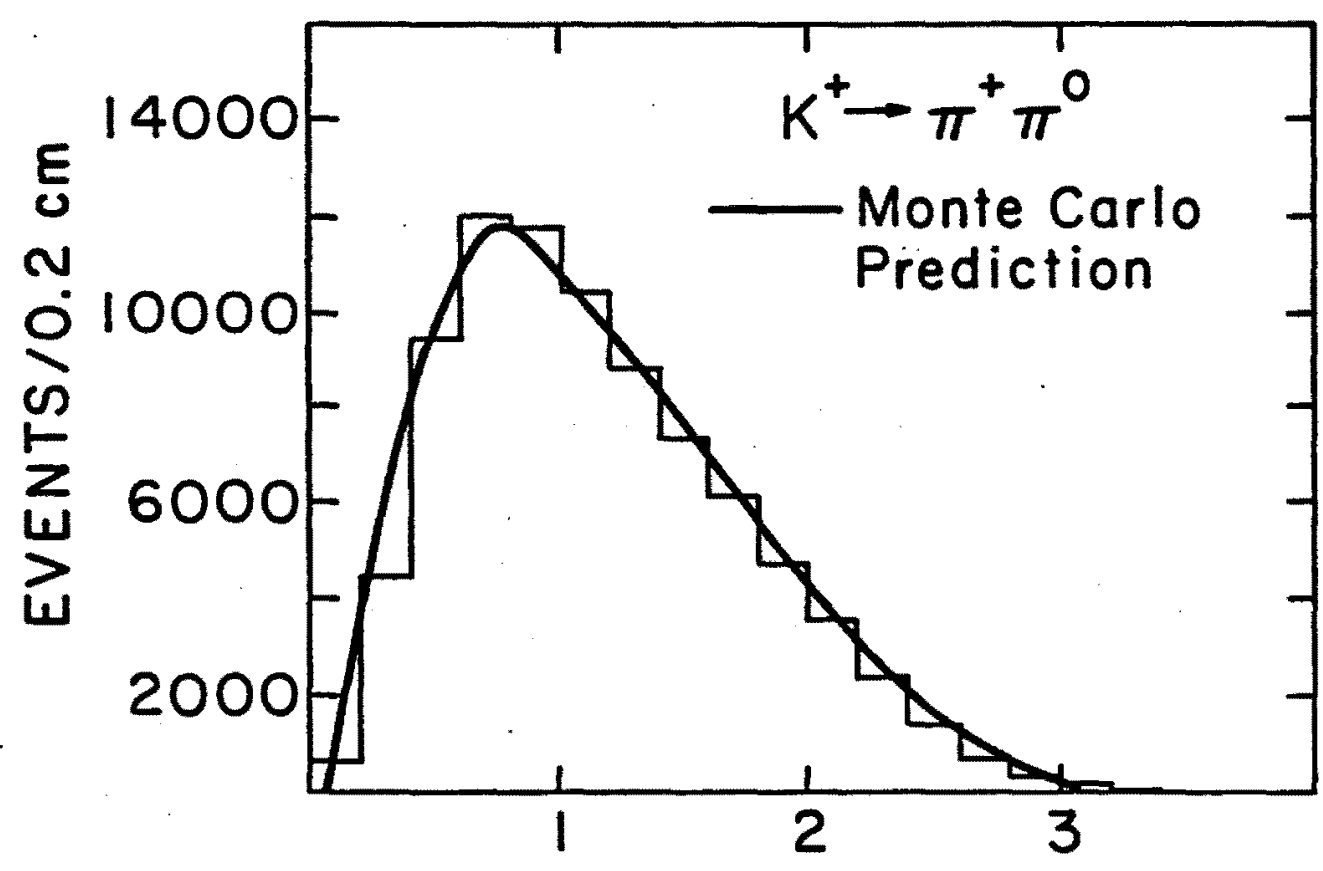

TRANSVERSE DISTANCE $(\mathrm{cm})$

Figure 36 


$$
\text { Asymmetry }=\left|\frac{E_{\text {Photon 1 }}-E_{\text {Photon 2 }}}{E_{\text {Photon 1 }}+E_{\text {Photon 2 }}}\right|
$$

The solid line in the figure is the Monte carlo generated curve. Since the $\pi^{\circ}$ is also a spin 0 object, the asymmetry distribution should be flat. The dropoff at higher asymmetries is due mainly to one of the photons from the $\pi^{\circ}$ falling below the $5 \mathrm{GeV}$ minimum energy cut.

The energy distribution for $\pi^{\circ}$ 's from $\mathrm{K}^{+} \rightarrow \pi^{+} \pi^{\circ}$ events is shown in Figure 38. The $\pi^{\circ}$ energy spectrum, which in the absence of acceptance effects would be flat, is strongly shifted towards high energy. Events with high energy $\pi^{+' s}$ (and hence low energy $\pi^{\circ} \mathrm{s}$ ) were vetoed since the charged pion was regarded as a noninteracting beam particle. This effect was mentioned before in connection with the angular distribution of the charged pion in the helicity frame.

The separation $\left.R\left(=\sqrt{\left(x^{2}+Y^{2}\right.}\right)\right)$ of the two photons from the $\pi^{\circ}$ in the LAC is shown in Figure 39a. The separation in the LAC in one view ( $X$ or $Y$ ) of the two photons is shown in Figure 39b. The peak at zero separation corresponds to a complete overlap in that view. In the case of complete overlap in one view, photon energies and positions were assigned by making use of the information in the other view. This was discussed in some detail in Chapter III. The solid 

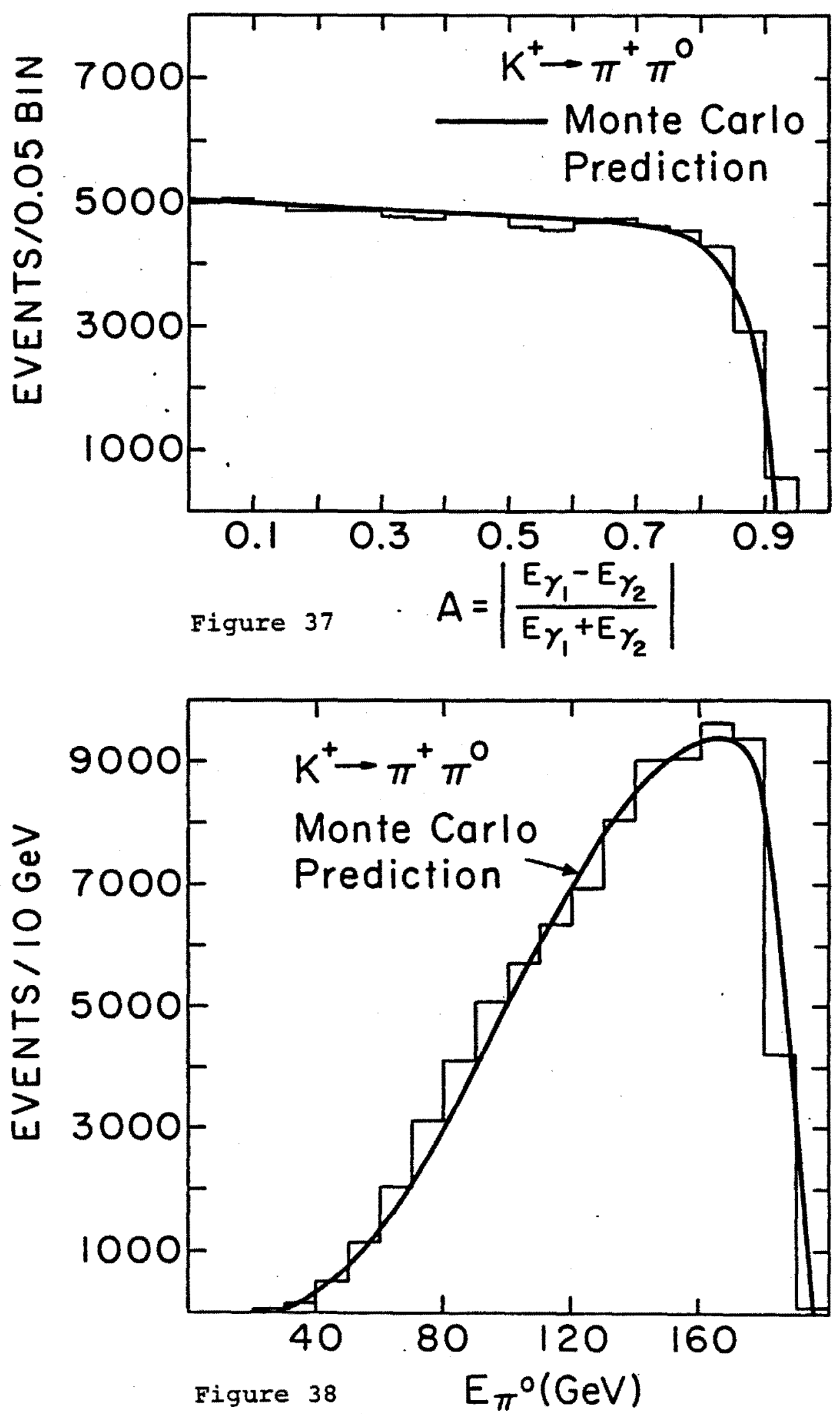


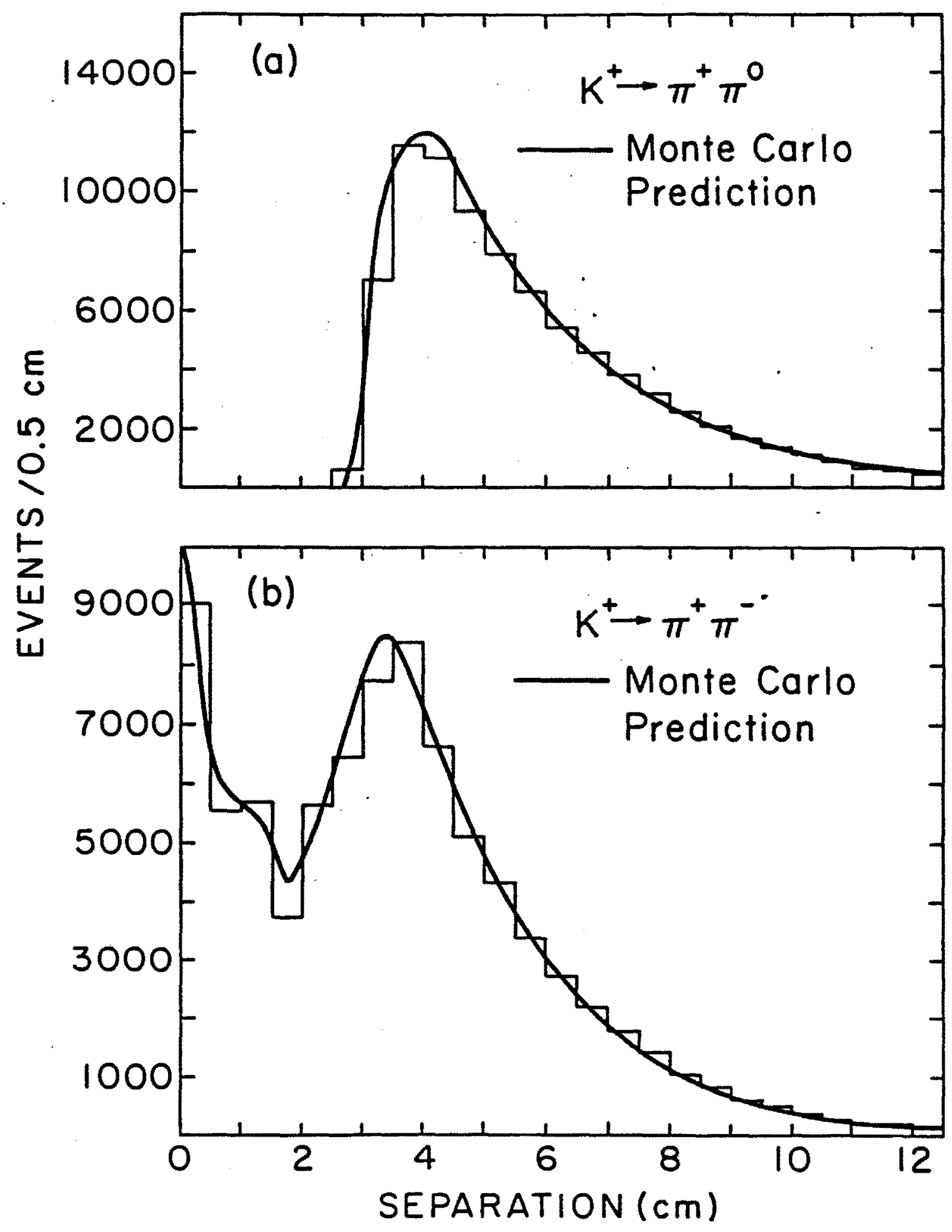

Figure 39 
Iine in both curves is the Monte Carlo prediction. The point emphasized by the last three figures is that the average $\pi^{\circ}$ energy in this experiment was high, and correspondingly, the average separation of the two photons was small. The presence of overlapping showers led to a degradation of the energy and position resolution for photons.

$$
\mathrm{K}^{+}+\mathrm{e}^{+} \pi^{\circ} v_{e} \text { events were generated in the same region of }
$$
the spectrometer as $\mathrm{K}^{+} \rightarrow \pi^{+} \pi^{\circ}$ decays. Using the positrons from from these Re3 decays, the position and energy resolution of the LAC, as generated by the Monte Carlo, were tested. A

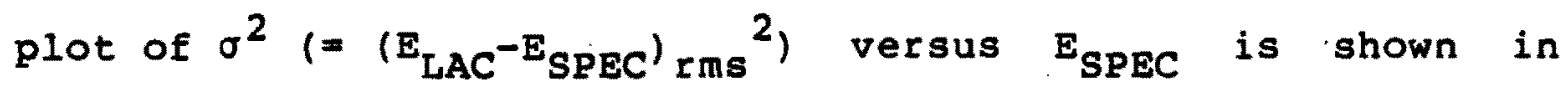
Figure 40. For comparison, the resolution function observed for the data, $\sigma^{2}=(.14 \sqrt{\mathrm{E}})^{2}+(.55)^{2}$, is plotted as a dashed line. Reasonable agreement is seen. A comparison of the position resolution of the LAC observed in the Monte Carlo and in the experimental data is shown in Figure 41 . For energies greater than. $35 \mathrm{GeV}$, good agreement is seen. For energies lower than this, the degradation of the resolution observed in the real data is much stronger than the degradation observed in the Monte Carlo data. The reason for this low energy discrepancy was mentioned in Chapter III. Having been bent by the analyzing magnet, low energy positrons entered the LAC at large angles. The large angles of incidence meant that fluctuations in the longitudinal development of the electromagnetic showers translated to 


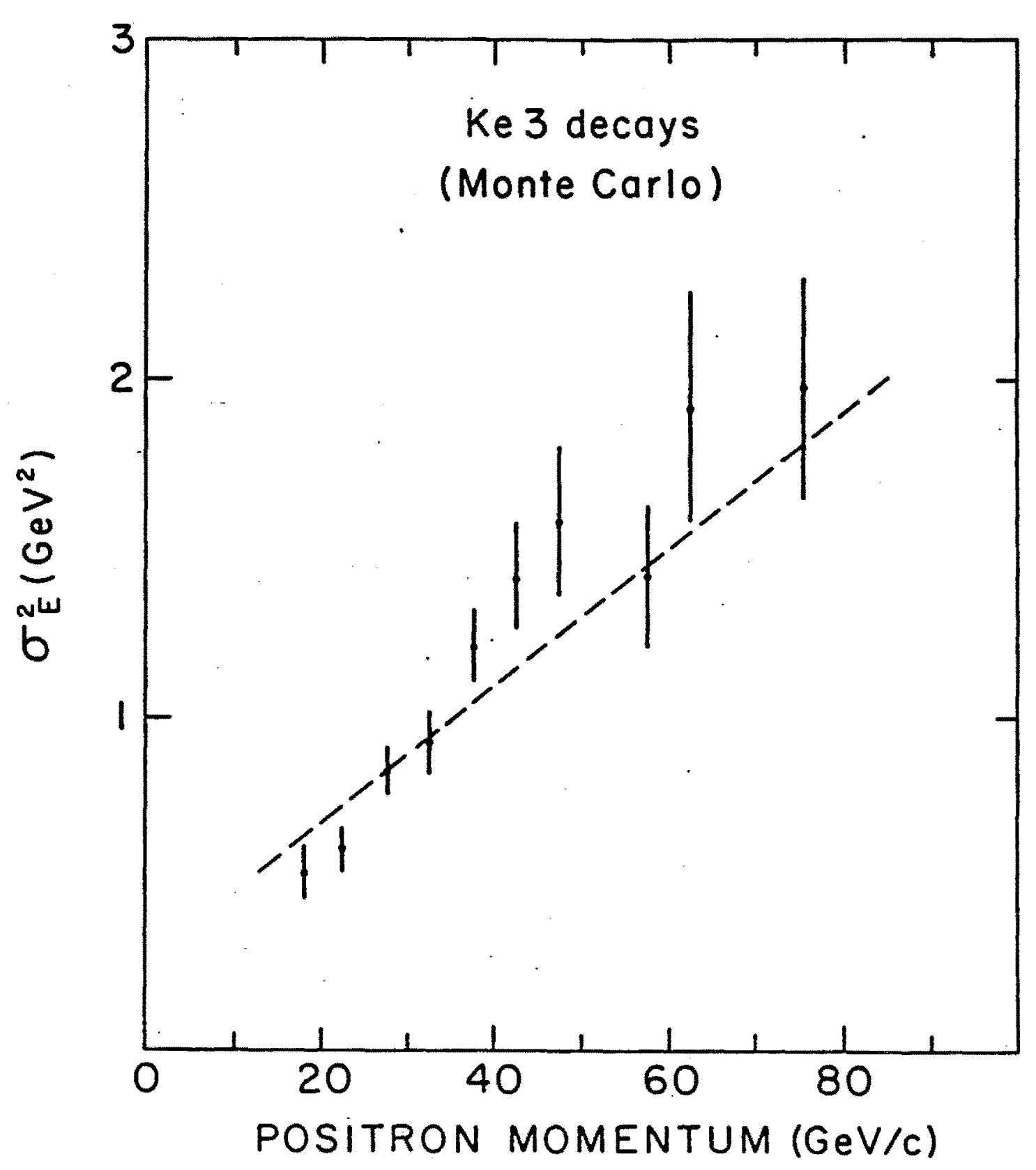

Figure 40 


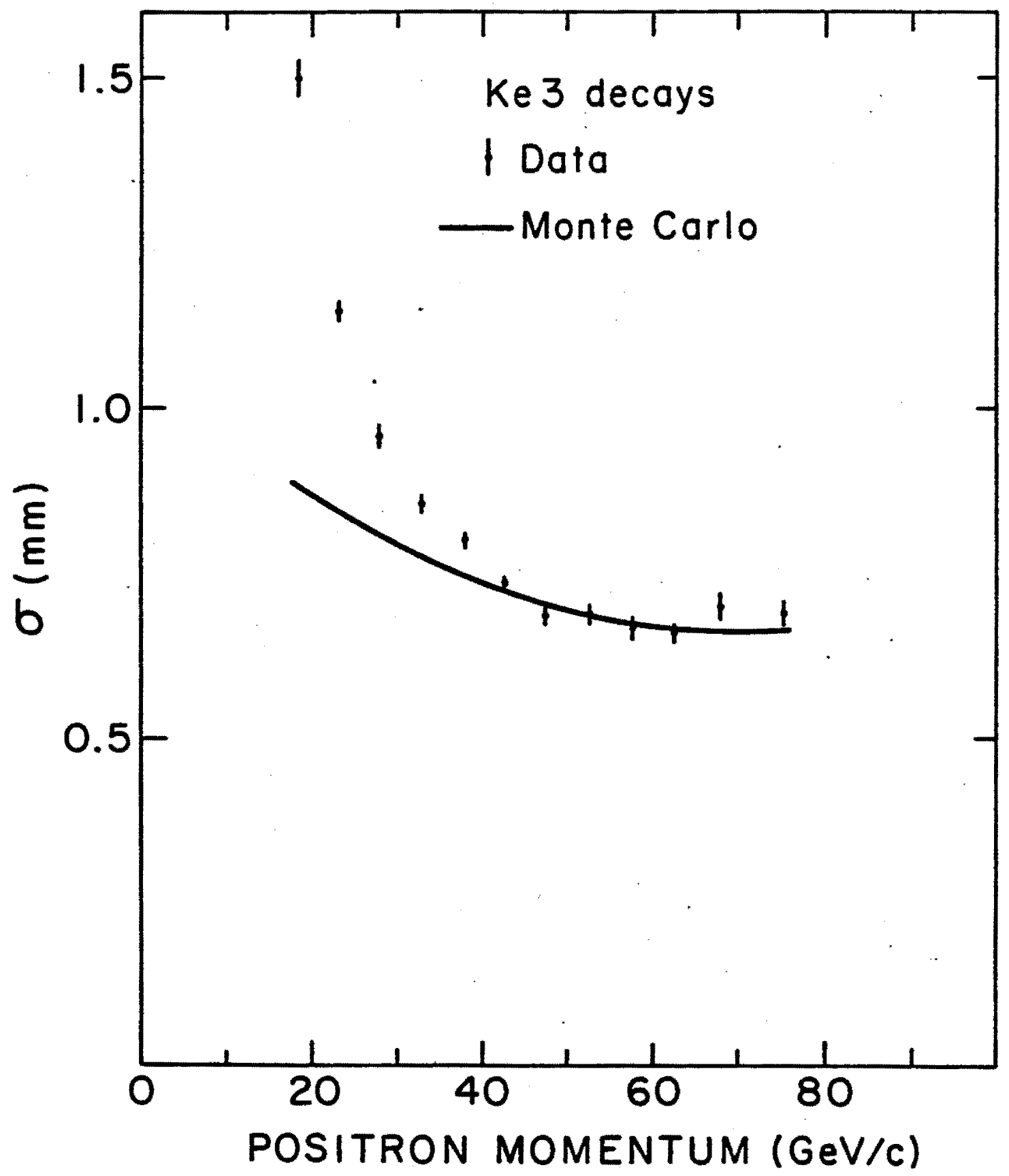

Figure 41 
fluctuations in the observed transverse positions of the showers. In the general E272 Monte Carlo, shower fluctuations were not introduced since the bulk of the data(all $\mathrm{K}^{+} \rightarrow \pi^{+} \pi^{\circ}$ and $\rho^{+} \rightarrow \pi^{+} \pi^{\circ}$ events) involved angles into the LAC of less than 10 milliradians. (As seen from the figure, no significant degradation is noticed for angles less than 1 GeV(size of momentum kick)/35 GeV $\simeq 30$ milliradians.) As a test, longitudinal variations in the shower development were inserted into the Monte Carlo for Ke3 decays(using a formula derived from Rossi's Approximation B [46]), and a degradation of the position resolution at low energies similar to that observed in the experimental data was seen. The position resolution at higher energies was unaffected.

The two photon mass distribution for $\mathrm{K}^{+}+\pi^{+} \pi^{\circ}$ decays and the Monte Carlo comparison are shown in Figure 42. The Monte Carlo distribution is somewhat narrower than the data; for the Monte Carlo $\sigma=4.7 \mathrm{MeV}$, for the experimental data $\sigma=5.5 \mathrm{MeV}$. The cause of this discrepancy is not clear. It may have to do with the difficulty of using an electromagnetic shower shape determined at 50 Gev over a wide range of energies. As mentioned earlier, the shower shape was used in the reconstruction program to separate neighboring photons. Since the Monte Carlo used the same shower shape for generation as was used in the reconstruction program, the resolution would necessarily be expected to be 


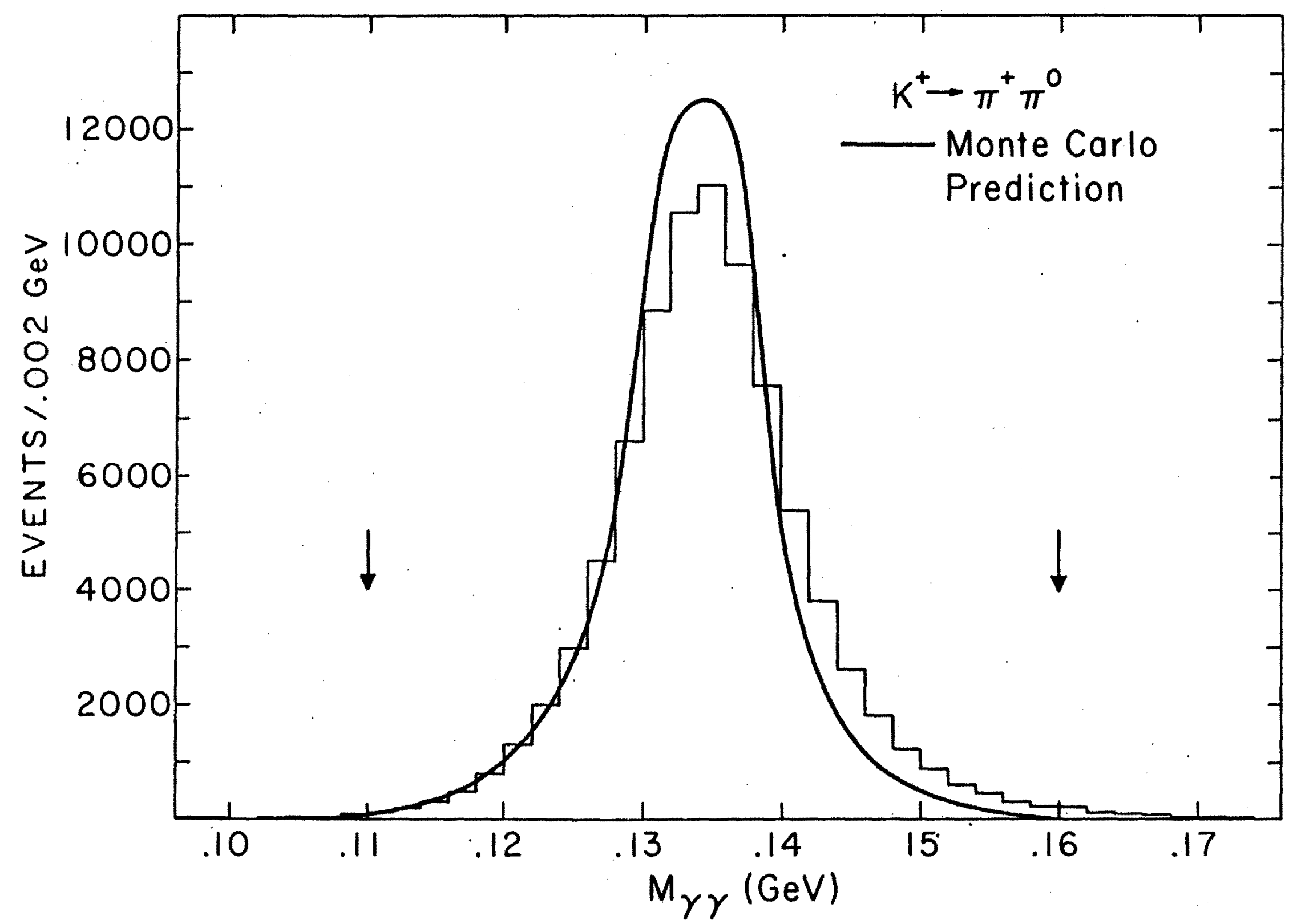


better. The $\pi^{\circ}$ mass cut used in the data analysis is shown in the figure by the two arrows.

Figure 43 shows the $\pi^{\circ}$ mass resolution plotted versus the $\pi^{\circ}$ energy, again for $\mathrm{K}^{+} \rightarrow \pi^{+} \pi^{\circ}$ events. The dashed curve is the mass resolution expected using the position and energy resolution observed with Re3 events. The solid curve is the Monte Carlo prediction. The mass resolution observed in the data was roughly a factor of 1.5 worse than was expected from the position and energy resolutions found for single showers. The Monte Carlo predictions agree more closely. (The events in this sample have been restricted to low asymmetry.) The discrepancy between the resolution found and the resolution expected points out the difficulty of working with two overlapping showers rather than single isolated showers.

The $\pi^{+} \pi^{\circ}$ mass distribution, from $\mathrm{K}^{+}$decays, is shown in Figure 44 along with the Monte carlo prediction. The Monte Carlo curve agrees fairly well with the data, although it is a bit narrower due to the better $\pi^{\circ}$ mass resolution seen in the Monte Carlo. The standard deviation for the data distribution is $8.5 \mathrm{Mev}$. The limits of the $\mathrm{K}^{+}$mass cut for the data analysis are shown by the arrows.

The $\pi^{+} \pi^{\circ}$ energy distribution is shown in Figure 45. The total energy of the event was reconstructed. Thus the 


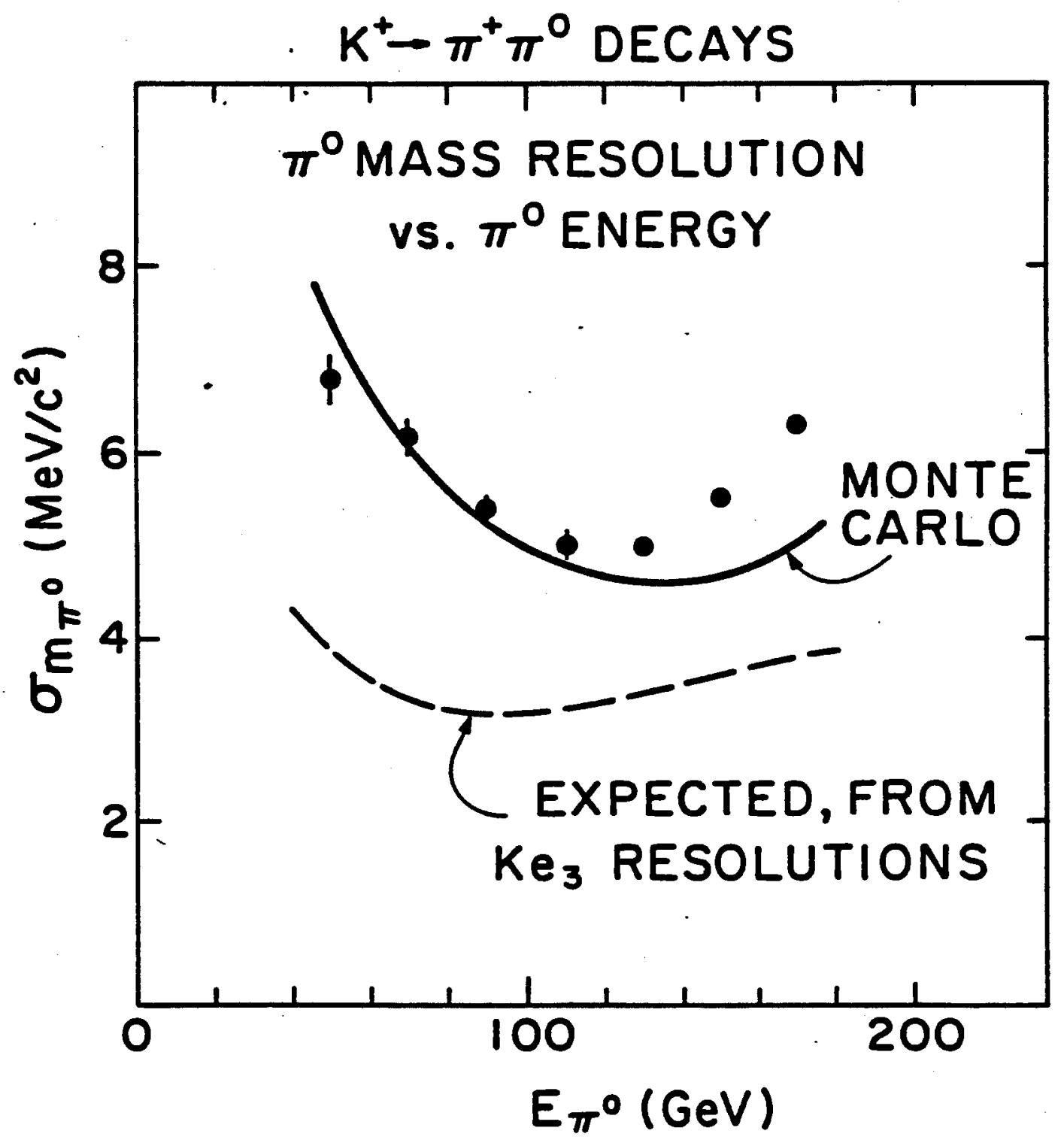

Figure 43 


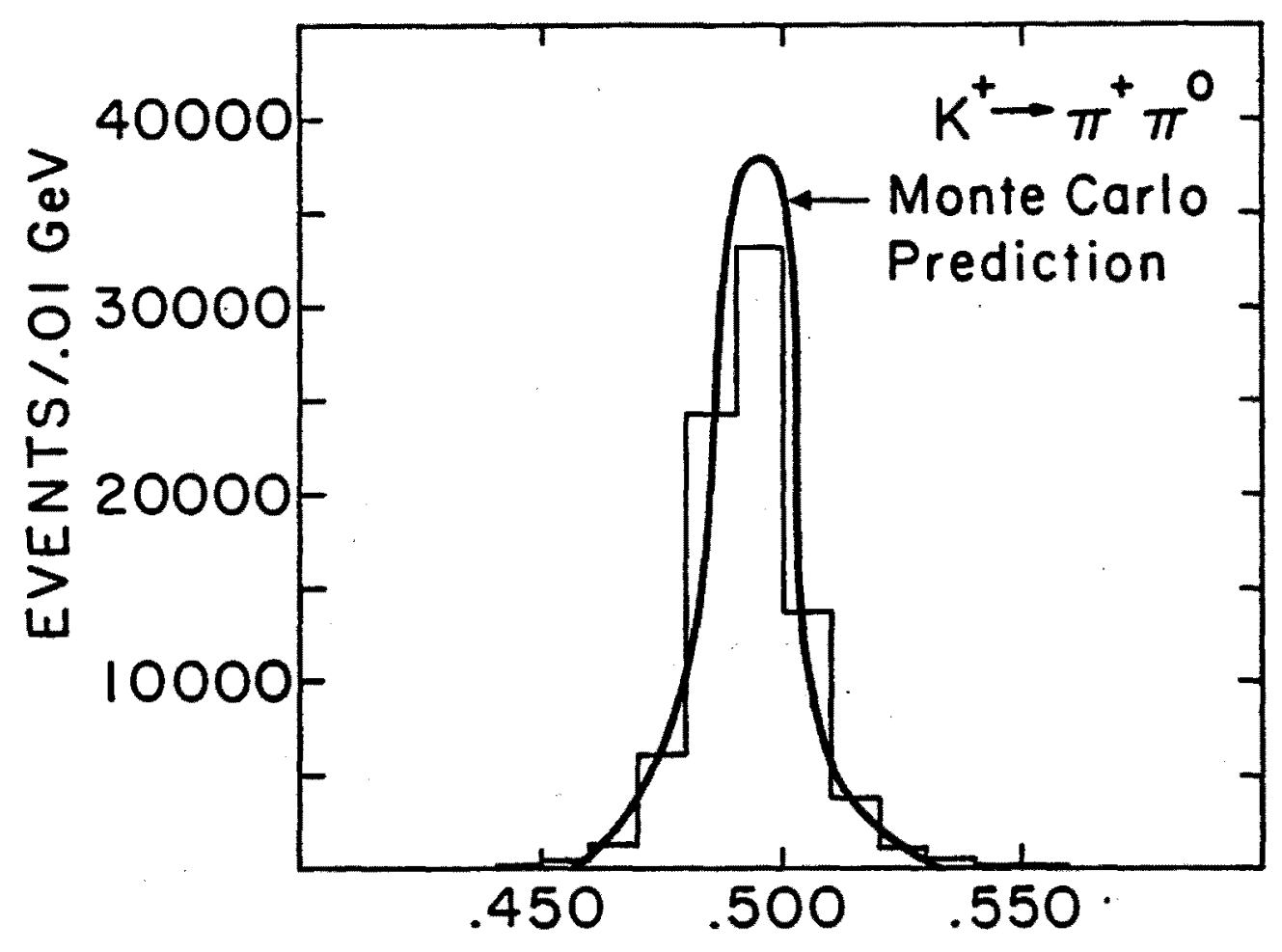

Figure 44

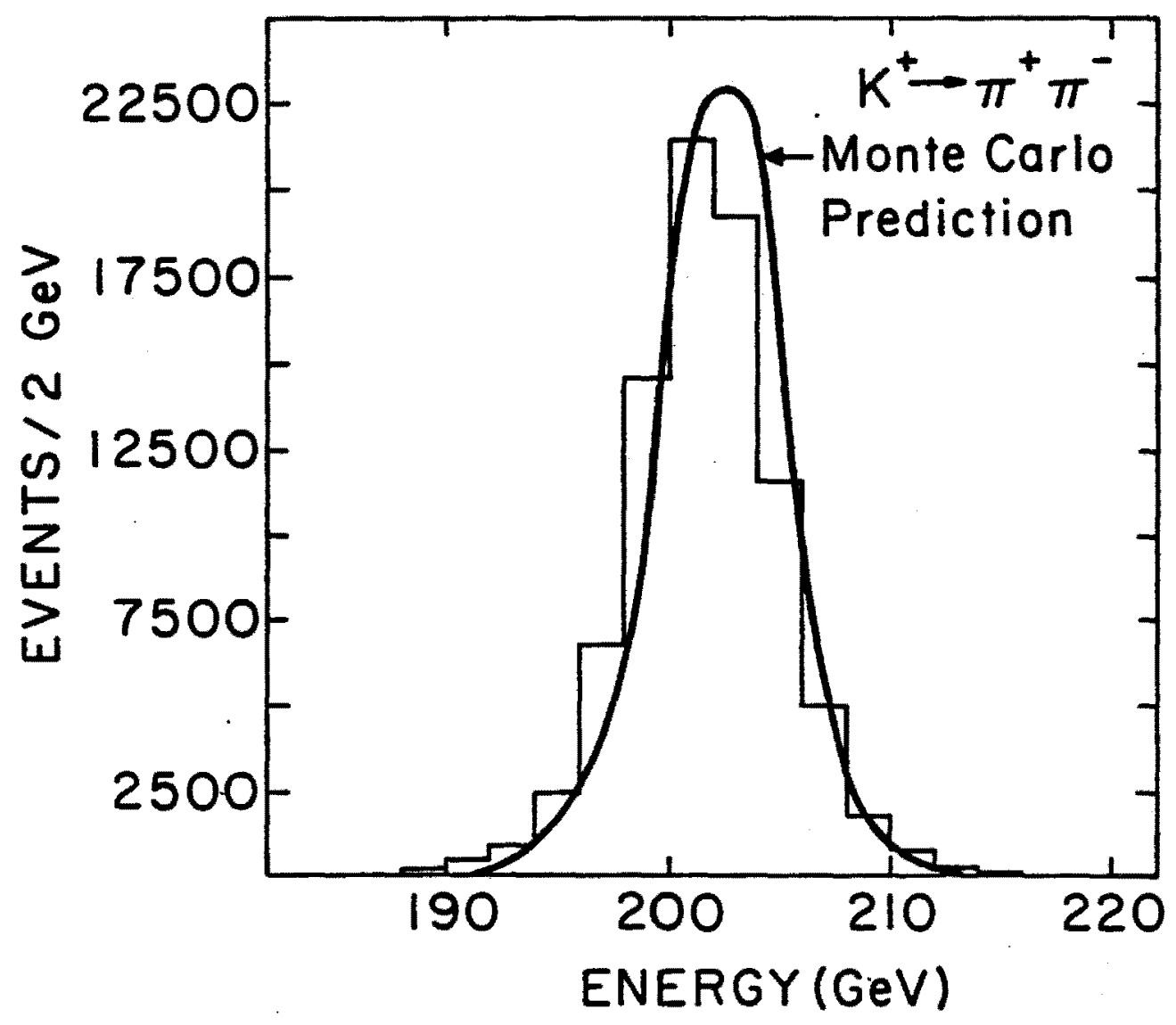

Figure 45 
width of the distribution comes from two sources: the resolution of the spectrometer, and the momentum bite of the incident beam. The limits of the energy cut used in the analysis are shown by the arrows.

One of the greatest values of the $\mathrm{K}^{+}+\pi^{+} \pi^{\circ}$ decays is in the knowledge they give of the $t$ resolution of the spectrometer. This point was discussed in Chapter III. If we assume a Gaussian resolution for the spectrometer, the $t$ distribution observed for $\mathrm{K}^{+}+\pi^{+} \pi^{\circ}$ decays has the form

$$
N(t)=N_{0} e^{-t / 2 \sigma^{2}}
$$

where $\sigma$ is the resolution in in transverse momentum $\left(p_{t}\right)$ of the spectrometer. (See Appendix B for more details.) The observed $t$ distributions of $k^{+} \rightarrow \pi^{+} \pi^{0}$ decays for the three different targets, and for target empty, are shown in Figure 46. The assumed exponential form works well at lower values of $t$. At higher values of $t$, the Gaussian approximation does not work as well. In addition, contamination from $\operatorname{Re} 3$ and Ku3 decays begins to become significant.

Multiple scattering in the target contributes a great deal to the observed t resolution. This is demonstrated by the much steeper $t$ slope seen with no target present. The differences in slope among the three targets are due to 


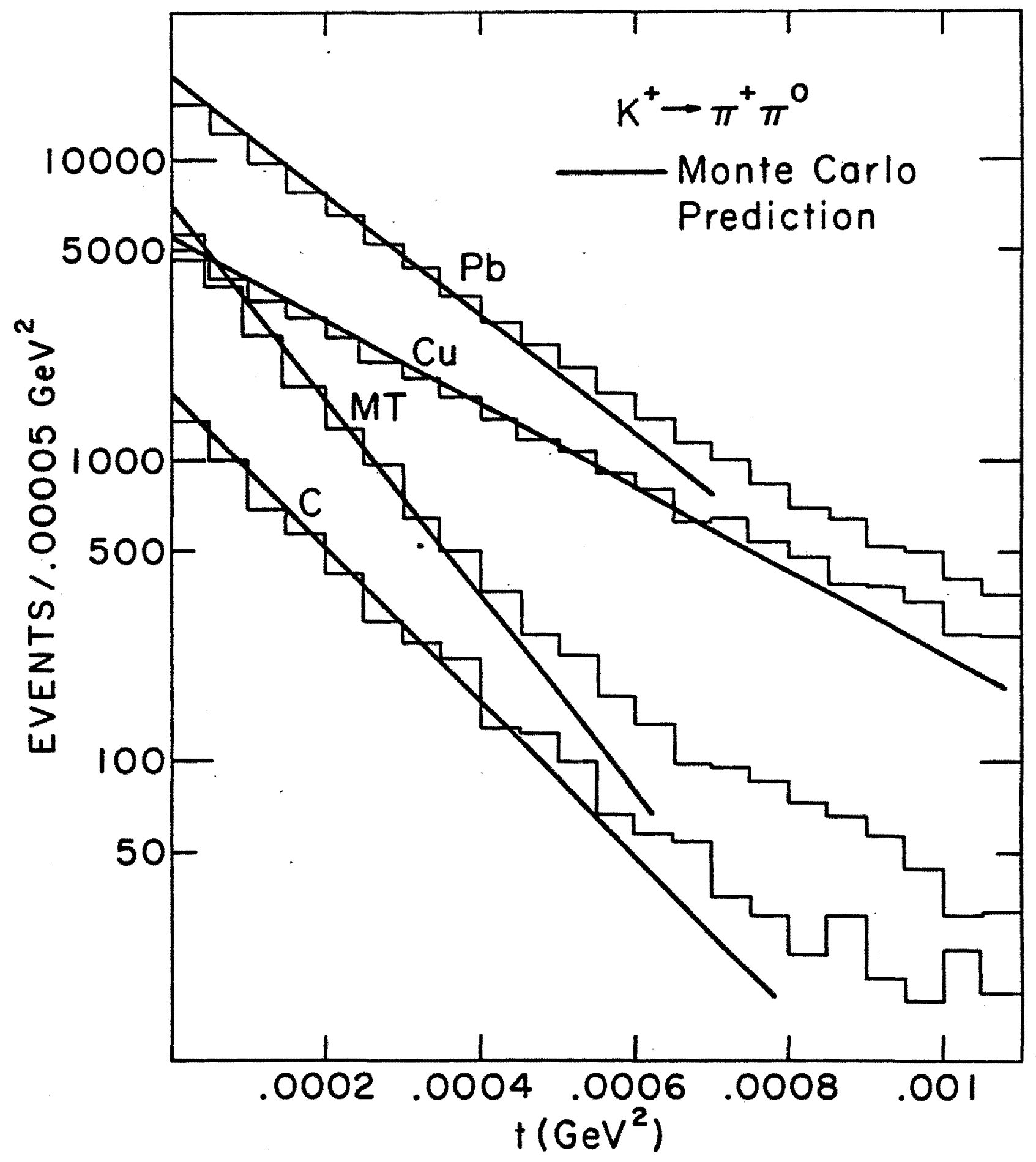

Figure 46 
differences in target thickness. The slopes seen in the Monte Carlo for the three targets and for no target are shown by solid lines. Agreement between the Monte Carlo and data is good, but again the resolution obtained in the Monte carlo is somewhat better. Since the Monte carlo is used to determine the $t$ resolution appropriate for $\rho^{+}$production, it is important to take into account any discrepancy. It was assumed that the $t$ resolution for $\mathrm{K}^{+}+\pi^{+} \pi^{\circ}$ decays could be written in the form:

$$
\sigma_{\text {Experimental }}^{2}=\sigma^{2} \text { Monte Carlo }+\sigma^{2} \text { Residual }
$$

A value for $\sigma^{2}$ Residual was obtained for every target. (See Tabie II.) Furthermore, it was assumed that $\sigma^{2}$ Residual had the same value for $\rho^{+}$events as for $k^{+}$decays. The quadratic sum of the Monte Carlo $\rho^{+}$resolution and the residual term was used as the "experimental" resolution appropriate for $\rho^{+}$ production. The effects of this assumption will be discussed in Chapter VI. 
Table II

$$
\text { Resolution in } \mathrm{p}_{t} \text { for } \mathrm{K}^{+} \rightarrow \pi^{+} \pi^{\circ}
$$

$\begin{array}{cccc}\text { Target } & \frac{\sigma_{\text {Monte }} \operatorname{Carlo}(\mathrm{MeV})}{\mathrm{C}} & 8.9 & \frac{\sigma_{\text {Data }}(\mathrm{MeV})}{\sigma_{\text {Residual }}(\mathrm{MeV})} \\ \mathrm{Cu} & 12.0 & 9.6 & 3.5 \\ \mathrm{~Pb} & 9.8 & 12.9 & 4.8 \\ \mathrm{MT} & 7.8 & 11.1 & 5.2 \\ & & 8.6 & 3.5\end{array}$




\section{Chapter V}

Normalization

A determination of the radiative width of the $\mathrm{p}^{+}$ meson involves, to first order, the determination of the cross section for $\rho^{+}$production. This can be seen from Equation (1.51). As a matter of definition then, the accuracy to which the radiative width can be determined is bounded by the accuracy to which the cross section can be determined. This chapter discusses the procedures used for normalizing the $\rho^{+}$cross section. As mentioned in the last chapter, the Monte Carlo provided a measure of the geometrical acceptance and trigger efficiency of the apparatus. By feeding the Monte Carlo generated events into the reconstruction program, the reconstruction efficiency of the off-line analysis was also determined. Other corrections had to be applied to take into account factors not inciuded in the Monte Carlo such as initial or final state absorption, Cerenkov counter contamination, and counter misalignment. The calculable corrections can be divided into two. categories: those relating to beam normalization, and those 
relating to corrections to the final state due to spectrometer effects. Non-calculable corrections, such as equipment readout failures,etc., were accounted for by making use of the normalization of the $\mathrm{K}^{+} \rightarrow \pi^{+} \pi^{\circ}$ decay. Correction factors for both $\rho^{+}$production and for $\mathrm{K}^{+}+\pi^{+} \pi^{\circ}$ decays are discussed in this chapter.

\section{A. Beam Normalization}

The definition of the incident bean flux was discussed in Chapter II. The beam flux for each particle type was further defined by requiring the appropriate signals from the Cerenkov counters. Misidentification of particle type was possible, mainly due to electronic noise in the phototubes. An estimate of the misidentification was made using presgure curves (Figures 8,9 , and 10) and the beam counting scalers. The kaon content in the pion beam was estimated to be about 0.58 and the pion content in the kaon bean was estimated to be 1.58. Correction factors were applied to the appropriate beam fluxes.

Past experience in the Ml beamline has shown the muon flux to be approximately 18 of the pion flux. [47] This figure is consistent with calculations of pion and kaon decay upstream of the Cerenkov region. Since muons registered as pions in the $\mathrm{K} 2$ Cerenkov counter, a correction factor needed 
to be applied to the pion beam flux. However, as discussed in Chapter II, approximately $2.2 \mathrm{~m}$ of beryllium was placed at the first focus in the beamline to enhance the $\mathrm{K}^{+}$fraction of the beam. The presence of the beryllium in the beamline had the side effect of enhancing the muon fraction of the beam since muons produced upstream of the first focus were not attenuated to any significant degree while the pion flux was reduced by a factor of several hundred. The muon contamination in the pion signal was measured using the beam triggers from a "straight thru" run in which the BM109 was turned off. [48] since no magnetic field was present, the beam particles were not bent into the beam hole in the LAC, but instead intersected the active area. The beam intensity was reduced to avoid any saturation effects in the LAC amplifiers. The number of proton, kaon, and pion absorption lengths presented by the LAC were calculated. Comparisons were made between the percentage of beam particles of each type that were expected to interact and the percentage that actually did interact. The proton and kaon measurements were consistent with calculations(see below about muon contamination in the kaon flux), but the pion comparison demonstrated the existence, at the $6 \%$ level, of a non-interacting component in the pion flux. This non-interacting component was interpreted as muon contamination and an appropriate correction was applied to the pion flux. 
Electrons in the beam will also have registered as pions in the Cerenkov counters. In Reference 47 , the electron contamination in the pion signal was measured to be 0.18. Since the presence of the beryllium in the beamline caused no electron enhancement, the 0.18 figure was used as a correction in this experiment.

Decays of incident kaons between the Cerenkov region and the spectrometer were simulated using a Monte Carlo. The decay $R^{+}+\mu^{+} v_{\mu}$ was estimated to reduce the kaon yield by about 0.48 . (The only decays effective in reducing the flux were those in which the decay muon was inside the beam acceptance.) In the study discussed in the previous paragraph, a muon contamination consistent with 0.48 was seen in the $\mathrm{R}^{+}$signal. A correction factor for this contamination was applied to the $\mathrm{R}^{+} \mathrm{flux}$. In a similar way the pion flux was reduced by the decay $\pi^{+}+\mu^{+} \nu_{\mu}$. The correction for this, of course, was included in the muon contamination factor mentioned before.

The amount of material downstream of the Cerenkov region, but upstream of the target, amounted to about $6 \times 10^{-3}$ proton absorption lengths. A correction was applied for incident particle absorption in this material. Also, interaction of the beam with this material could have created more than one beam track. To prevent ambiguities, events 
with more than one beam track were not considered for analysis. A correction was applied for this to the beam count. The effects discussed above are summarized in Table IIIa.

Events, otherwise good, could have been killed due to the accidental firings of the various veto counters in the experiment. The veto counters could have fired because of random, noise in the phototubes, delta ray emission from the charged particles in the event, or misalignment of the veto counters. (As mentioned earlier, the B counter was found to be misaligned during part of the run, vetoing events in which one of the particles in the final state happened to hit the counter.) A correction factor for this type of occurence was calculated using Beam trigger events. The Beam trigger was a minimum bias trigger, depending on none of the veto counters for its definition. The rate of firing of the veto counters for the Bean triggers was measured, and correction factors were applied for each target and incident particle type.

The $\mathbf{s}$ counter was set up downstream of the target so that all charged particles in the events of interest would pass through it. A signal from the $s$ counter corresponding to one or more charged particles in the event was required for the Rho trigger. During part of the run, the counter was bent slightly away from the beam region and it was possible 


\section{Table IIIa}

Correction Factors to Beam Normalization

$$
\text { (effects independent of target) }
$$

\section{Beam Type}

\begin{tabular}{|c|c|c|}
\hline & $\pi^{+}$ & $\underline{\mathrm{K}^{+}}$ \\
\hline $\begin{array}{l}\text { Cerenkov } \\
\text { misidentification }\end{array}$ & .995 & .987 \\
\hline $\begin{array}{l}\text { Muon } \\
\text { contamination }\end{array}$ & $.94 \pm .015$ & - \\
\hline $\begin{array}{l}\text { Electron } \\
\text { contamination }\end{array}$ & .999 & \\
\hline $\begin{array}{l}\text { Decay of beam } \\
\text { particle } \\
\text { downstream of } \\
\text { Cerenkov region }\end{array}$ & $\underbrace{\star *}$ & .996 \\
\hline $\begin{array}{l}\text { Absorption of } \\
\text { particle in } \\
\text { upstream material }\end{array}$ & .9995 & .9996 \\
\hline
\end{tabular}


for charged particles from good events to miss the counter. This effect was also studied using Beam triggers and the resulting factors were used to correct the normalization. The correction factors to the beam normalization due to the above effects are presented in Table IIIb.

\section{B. Spectrometer Related Corrections}

Event loss due to absorption of the initial or final state in the target was possible. For $\mathrm{K}^{+}+\pi^{+} \pi^{\circ}$ decays, only absorption of the incident $\mathrm{K}^{+}$needed to be calculated since $\mathrm{R}^{+}$decays in the target region (or upstream) were not considered in the normalization. For Reaction (1.1) , a convolution of the absorption in the target of the incoming and outgoing states was necessary. For each target, the data of A.S. Carroll et al. [49] was used to calculate the number of absorption lengths present for each particle type. Photon absorption was calculated using the formula exp(-7/9t), where $t$ is the thickness of the target in radiation lengths. (For high energy photons, the absorption length(determined by pair production) can be written as $9 / 7 t_{0}$; where $t_{0}$ is the radiation length. [50]) Correction factors for the above effects were calculated and applied to the cross sections.

The spectrometer presented as much material, or more, as the different targets. Correction factors for final state 


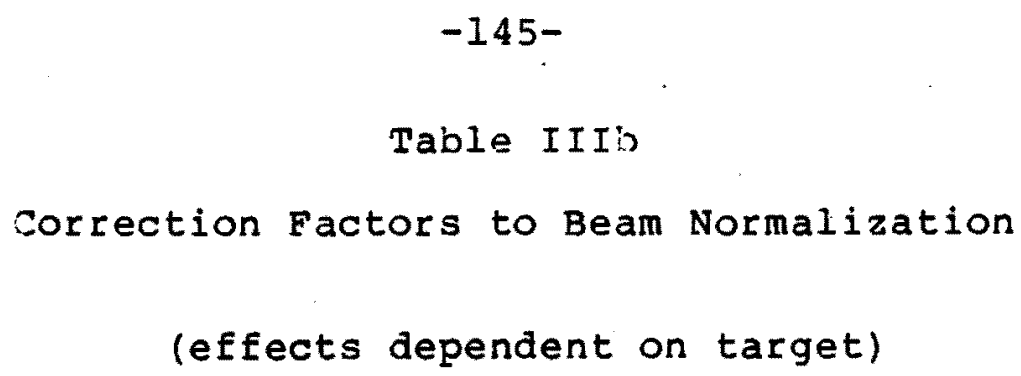


absorption were calculated in a manner similar to that described above. The material in the acceptance of the final state(helium bags, chambers, scintillation counters,etc.) was determined and the number of pion and kaon absorption lengths calculated, again using the data of Carroll et al. The number of radiation lengths in the above material was also calculated and corrections were applied for final state photon absorption.

Hadrons, interacting in the LAC,could sometimes mimic photons(although in general the characteristics of their energy deposition are sufficiently different to allow discrimination). As mentioned in Chapter III, showers in the LAC that had a charged track within $2.5 \mathrm{~cm}$ were designated as charged-photon overlaps and flagged as possible hadron or electron) candidates. If a hadron shower did not have a charged track within $2.5 \mathrm{~cm}$, and its longitudinal energy deposition was consistent with that of a photon, it was called a photon. Large fluctuations in hadron shower development in conjuction with large entrance angles in the LAC made it possible for the reconstructed shower position and the charged track position to differ by more than $2.5 \mathrm{~cm}$. A similar argument was applied to electron showers in Chapter IV. A study of $\mathrm{R}^{+} \rightarrow \pi^{+} \pi^{\circ}$ decays found that 2.28 of the events contained an extra photon. Since both $\mathrm{R}^{+} \rightarrow \pi^{+} \pi^{\circ}$ and $\rho^{+}+\pi^{+} \pi^{\circ}$ events were constrained in the analysis to have only two 
Table IVa

Correction Factors to Cross Section

(effects dependent on target)

\section{Target}

$\underline{\mathrm{C}} \quad \underline{\mathrm{Cu}} \quad \underline{\mathrm{Pb}}$

$\begin{array}{llll}\text { Incident } \mathrm{K}^{+} & 1.035 \pm .0001 & 1.033 \pm .0001 & 1.0054 \pm .0001 \\ \begin{array}{l}\text { absorption in } \\ \text { target }\end{array} & & & \\ \begin{array}{l}\text { Incident } \pi^{+} \text {and } \\ \text { outgoing } \pi^{+} \pi^{\circ} \\ \text { absorption } \\ \text { in target }\end{array} & 1.13 \pm .004 & 1.431 \pm .003 & 1.184 \pm .003 \\ & & & \\ & & & \\ \text { * specific to } . \mathrm{k}^{+} \rightarrow \pi^{+} \pi^{\circ} & & \end{array}$


$-148-$

Table IVb

Correction Factors to Cross Section

(effects independent of target)
$\mathrm{K}^{+}$absorption
1.00185
in $S$ counter
1.0021
$\pi^{+}$absorption
in $S$ counter
$\pi^{\circ}$ absorption.
in $S$ counter ${ }^{*}$
1.0072
$\pi^{+}$absorption
in spectrometer
1.024
$\pi^{\circ}$ absorption
1.132
in spectrometer
3 photon
correction
$1.022 \pm .005$
of $\pi^{\circ}$ decay
1.0116
* specific to $\mathrm{K}^{+} \rightarrow \pi^{+} \pi^{\mathrm{O}}$
** specific to $\rho^{+}+\pi^{+} \pi^{\circ}$ 
photons, the presence of a third photon resulted in the event being thrown out. A correction factor was applied to the data for this effect.

Since the $\pi^{\circ}$ has a .0115 branching ratio to $\mathrm{e}^{+} \mathrm{e}^{-} \gamma$ (Dalitz decay), a $1.15 \%$ correction was applied to both $\mathrm{K}^{+} \rightarrow \pi^{+} \pi^{\circ}$ and $\rho^{+} \rightarrow \pi^{+} \pi^{\circ}$ rates. The decay of final state charged pions in the spectrometer was investigated with the Monte Carlo and found to lead to a 0.35 correction for both the $\mathrm{K}^{+}$ and $\rho^{+}$events. The correction factors for spectrometer effects are summarized in Tables IVa and IVb.

\section{C. $\mathbf{k}^{+}$Decay Normalization}

The above correction factors, and those obtained from the Monte Carlo, were tested by comparing the expected rate for the decay $\mathrm{K}^{+}+\pi^{+} \pi^{\circ}$ to the rate actually seen. The comparison was carried out as follows. Using the incident kaon beam flux, the number of kaons expected to decay in the mode $\pi^{+} \pi^{\circ}$ in the region $1 \mathrm{~m}$ to $3.2 \mathrm{~m}$ downstream of the target was calculated. The number of kaons reconstructed in this region was multiplied by the correction factors mentioned in this chapter and by the correction factors determined by the Monte Carlo and reconstruction program. For both Monte Carlo and data $\mathbf{K}^{+} \rightarrow \pi^{+} \pi^{\circ}$ events, the following cuts were applied: 
1. Rho trigger

2. 1 beam track

3. Kaon Cerenkov

4. I track in final state

5. 2 photons in final state

6. no charge-photon overlaps with $E / P$ in the range 0.9-1.1 (This was applied to eliminate contamination from $R^{+} \rightarrow e^{+} \pi^{\circ} \nu_{e}$ decays.)

7. Decay vertex in the normalization region

8. Reconstructed energy in the range 192-212 GeV

9. Reconstructed $t$ less than .005 (GeV/c) ${ }^{2}$

10. Reconstructed $\pi^{\circ}$ mass in the range .110-.160 GeV

11. Reconstructed $\mathrm{K}^{+}$mass in the range $.450-.550 \mathrm{GeV}$

Some of these cuts were discussed in the last chapter. Cuts $1,2,4,5,6,8$ and 10 are common to the $\rho^{+}$ 
analysis. The $\mathrm{K}^{+}$decay normalization factors for each target are shown in Table $v$. Within errors, they all agree. As will be seen in the next chapter, these factors were applied as a correction to the $\rho^{+}$cross section. 
Table V

$$
\mathrm{K}^{+} \rightarrow \pi^{+} \pi^{\circ} \text { Normalization Factors }
$$

Target

C

$\mathrm{Cu}$

$\mathrm{Pb}$
Factor

$1.16 \pm .03$

$1.13 \pm .02$

$1.14 \pm .02$ 


$$
\rho^{+} \text {Data }
$$

A. Introduction

In this chapter the data for the reaction

$$
\pi^{+}+(A, Z)+\rho^{+}+(A, Z)
$$

will be presented and the results of fits to the mass and $t$ distributions will be discussed. Comparisons of the data to the Monte Carlo generated distributions will be made and the overall normalization of the cross section will be discussed in more detail.

Evidence that reaction $(1.1)$ is dominated by $\rho^{+}$ production is shown in Figures $47 \mathrm{a}, 47 \mathrm{~b}$, and $47 \mathrm{c}$ where the $\pi^{+} \pi^{\circ}$ mass distribution is plotted for the lead,copper and carbon targets respectively. For these distributions, cuts have been applied to the total energy, interaction vertex, and $\pi^{\circ}$ mass. For each of the targets, a proninent peak is 
$-154-$
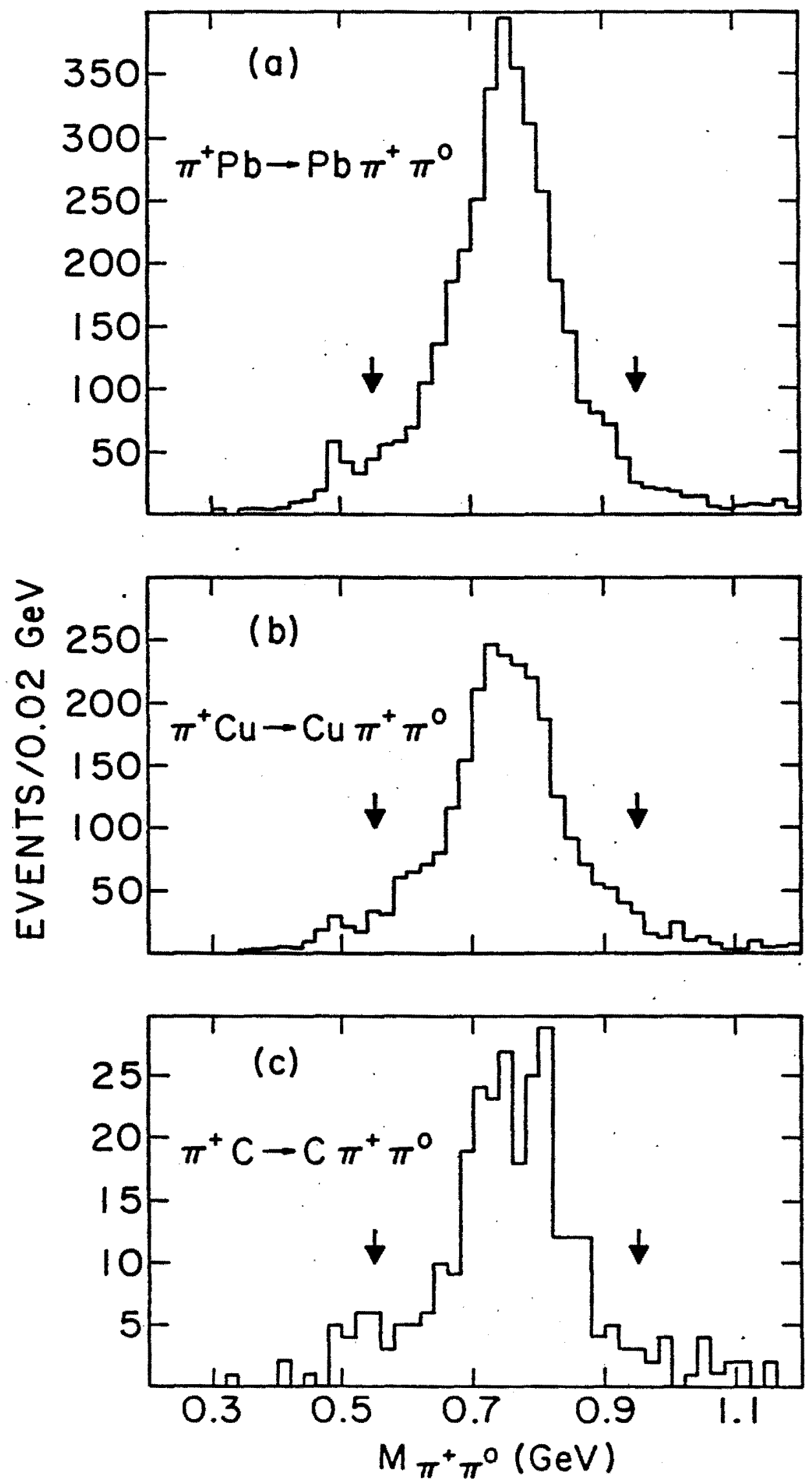

Fiqure 47 
seen in the $\rho^{+}$region. A secondary peak can also be noticed around $.500 \mathrm{GeV}$. This peak is due to $\mathrm{K}^{+}+\pi^{+} \pi^{\circ}$ decays in which the incident $\mathrm{K}^{+}$was identified as a $\pi^{+}$due to inefficiencies in the cerenkov system. To avoid the region of $\mathrm{k}^{+}$ contamination, $\rho^{+}$candidates with a mass below $.550 \mathrm{GeV}$ were not considered in the analysis. An upper mass cut of .950 GeV was also applied. The $\pi^{+} \pi^{\circ}$ mass range used in the analysis is indicated in Figure 47 by the two arrows. The loss of events (about 108 ) due to the $\rho^{+}$mass cut was corrected for using the line shape of the $\pi^{+} \pi^{\circ}$ mass distribution. This will be discussed in more detail in Section $D$.

B. Data and Comparisons to the Monte Carlo Model

Simulated events for reaction (1.1) were produced using the Monte Carlo model discussed in Chapter 4. The events were generated with a $t$ of near $0\left(1.0 \times 10^{-10} \mathrm{GeV}^{2}\right)$ and with a Breit-wigner mass distribution using the world averages for the resonance mass and width. (Again, for convenience's sake when discussing values of $t$, I'll refer to it as a positive number. For $t$, read $|t|$.$) The events were$ generated with a $t$ of near 0 both for the sake of simplicity and to calculate the expected resolution of the spectrometer for $\rho^{+}$production(as discussed in Chapter 4 ). The acceptance of the spectrometer for $\rho^{+}$production was checked and found 
to be $t$ independent up to a $t$ of $0.1 \mathrm{Gev}^{2}$. The events were also generated with a $\sin ^{2} \theta \sin ^{2} \phi$ angular distribution in the Gottfried-Jackson frame, as expected for a spin 1 resonance. The $\rho^{+}$mesons were produced at the target, allowed to decay, with the decay products propagating through the spectrometer. Events in which decay particles left the geometrical acceptance of the spectrometer were disregarded. Hits were generated in the chambers for charged tracks and showers were generated in the LAC for photons. Positions and, energies were smeared with the resolutions observed in the data. The chamber and LAC information was then passed to the same reconstruction program used for real data events. The Monte. Carlo events generated in this manner were used to estimate the geometric and trigger acceptances of the spectrometer and also the efficiency of the reconstruction program. As will be seen in section $C$, these factors were used in the calculation of the $\rho^{+}$cross section. The Monte Carlo events were also useful in that a comparison of Monte Carlo distributions to data distributions showed the degree to which the experiment was understood. In the following figures, unless otherwise noted, the data appear in histogram form while the reconstructed Monte Carlo results appear as solid curves.

The reconstructed interaction vertex distribution for $\rho^{+}$events is shown in Figure 48a. Cuts on total energy, $\pi^{\circ}$ 


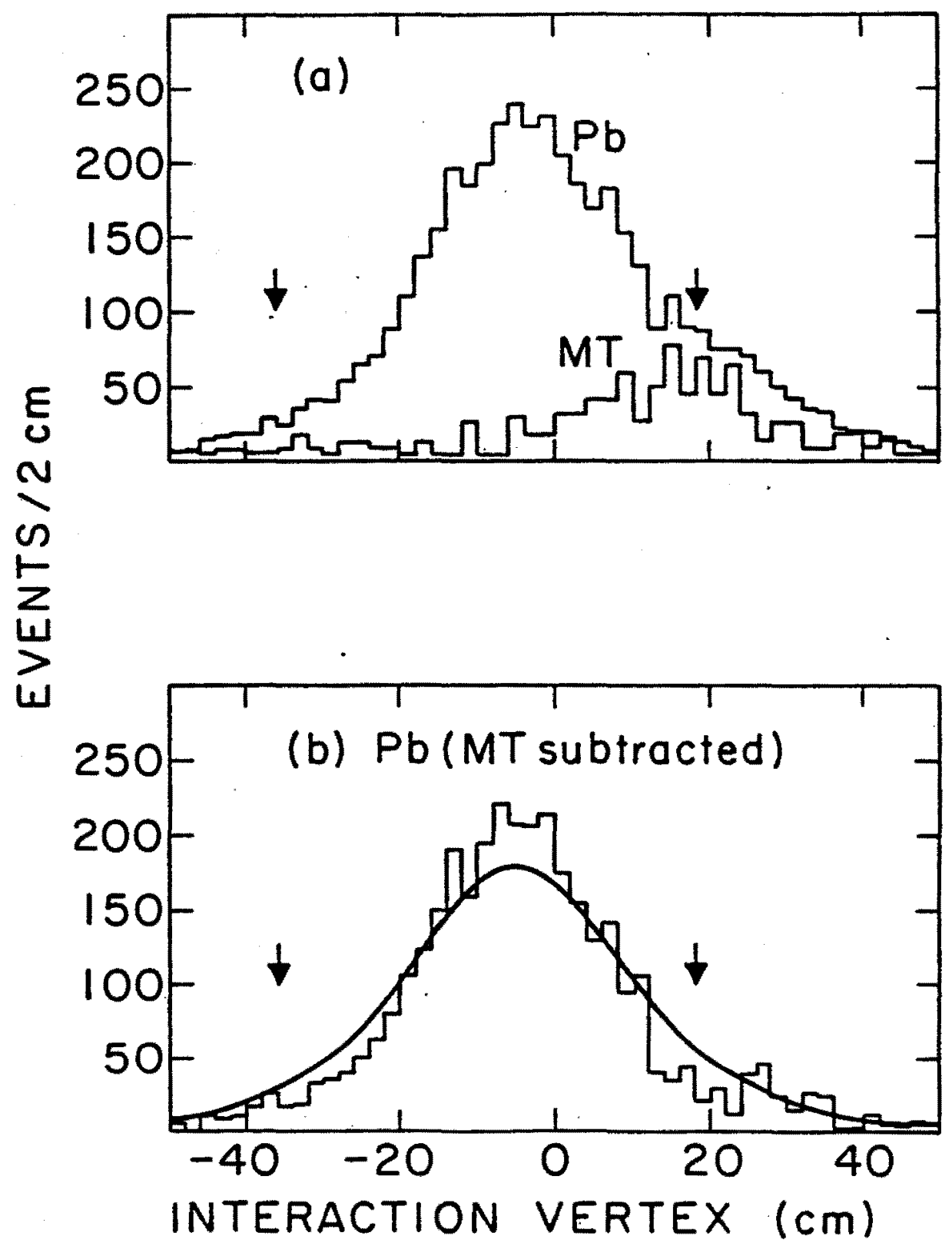

Figure 48 
mass, and $\rho^{+}$mass have been applied to the events in this distribution. The larger histogram shows the vertex distribution for the lead target while the smaller shows the. normalized(to the lead target beam count) distribution for no target present. From the figure it is apparent that most of the background from the target empty is due to interactions downstream of the target region. There are two reasons for this: more material is present downstream of the target and inţeractions upstream of the target tend to be eliminated by the target veto counters. To minimize the effect of the target empty subtraction, only those events in the region between the two arrows were used for further analysis. This cut results in a loss of real events of about $13 \%$. The loss wais corrected for using the Monte carlo. The lead target interaction vertex distribution, now with target empty subtracted, is shown in Figure $48 \mathrm{~b}$ and compared with the Monte Carlo prediction. The Monte Carlo distribution is seen to be somewhat broader than that found in the data. since a severe cut on the interaction vertex is needed to reduce the target empty background, the Monte Carlo overestimates the loss of events by about $8 \%$. This overestimation was studied and found to be the same for each target. A correction factor for this effect is included in. Table VII.

The distribution of the decay angle of the $\pi^{+}$from the $\rho^{+}$decay in the Gottfried-Jackson frame is shown in 
$-159-$
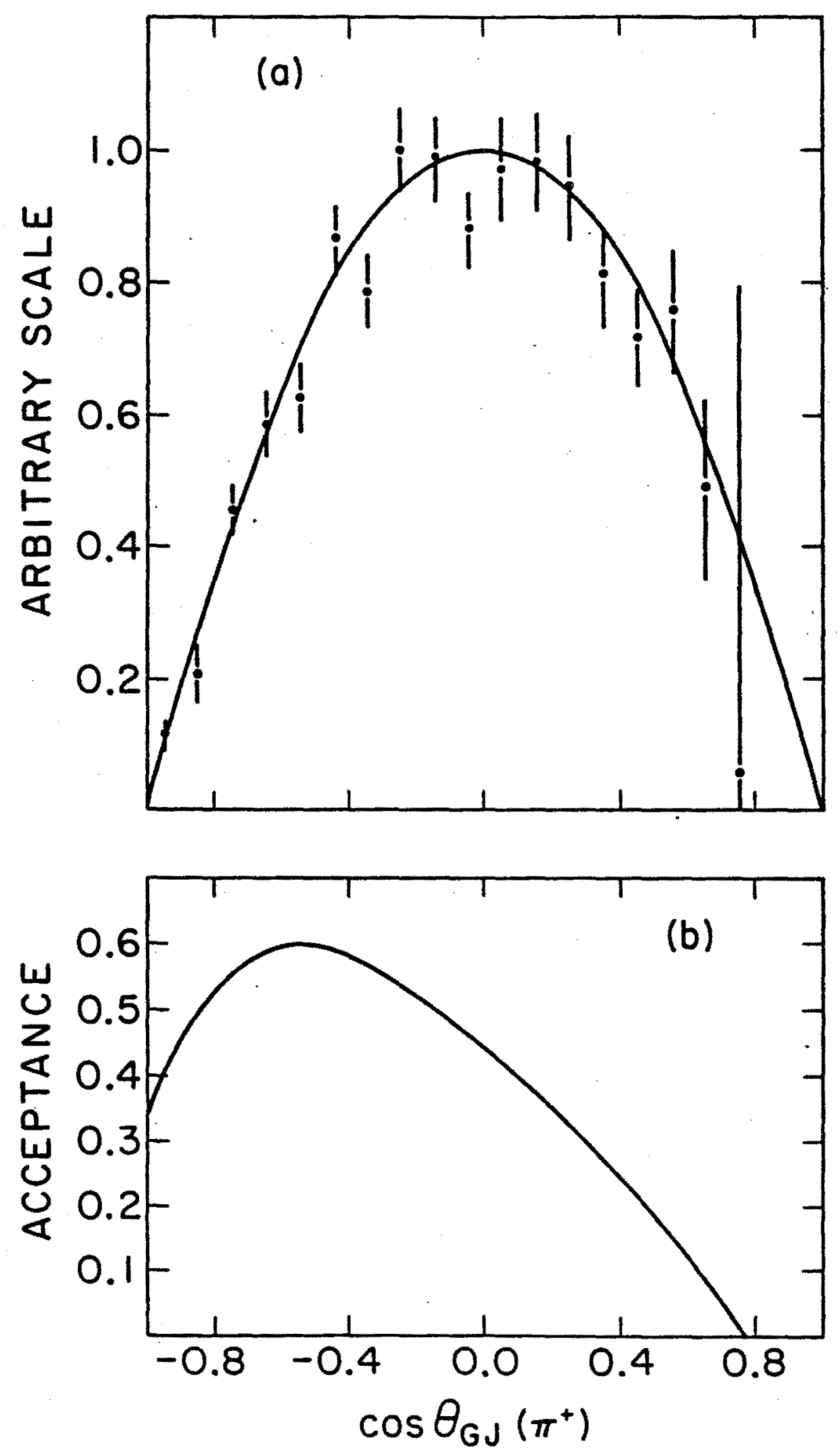

Figure 49 
Figure $49 \mathrm{a}$ for the lead target. Cuts on energy, interaction vertex, $\pi^{\circ}$ mass, and $\rho^{+}$mass have been applied. In addition the events have been restricted to have $t$ less than .002 $\mathrm{GeV}^{2}$. The events in Figure $49 \mathrm{a}$ have also been corrected for target empty background and for geometric acceptance and reconstruction efficiency. The results are in excellent agreement with the $\sin ^{2} \theta$ dependence (solid curve) expected for the decay of coherently produced $\rho^{+}$mesons. In Figure 49b, the combined geometric acceptance and reconstruction efficiency is shown as a function of $\cos \theta_{G J}$. The rapid falloff at forward angles is due to the effects of the various veto counters in the experiment, as discussed before.

The $\phi$ distribution of the $\pi^{+}$in the Gottfried-Jackson frame is shown for two different $t$ cuts in Figures 50a and 50b. At small values of $t$ the $\phi$ dependence is dominated by the resolution of the spectrometer. This is demonstrated in the two figures where the expected $\sin ^{2} \phi$ dependence is much more evident in the higher $t$ region.

The good agreement of the $\cos \theta_{G J}$ distribution with the expected $\sin ^{2} \theta_{G J}$ shape indicates that inelastic background is not a problem at small t. . The most. likely source of inelastic background would be from coherent $\mathrm{A}_{1}{ }^{+}+\pi^{+} \pi^{\circ} \pi^{\circ}$ production in which one of the $\pi^{\circ} \mathrm{s}$ was lost. Background from this source would introduce a $\cos ^{2} \theta$ component 


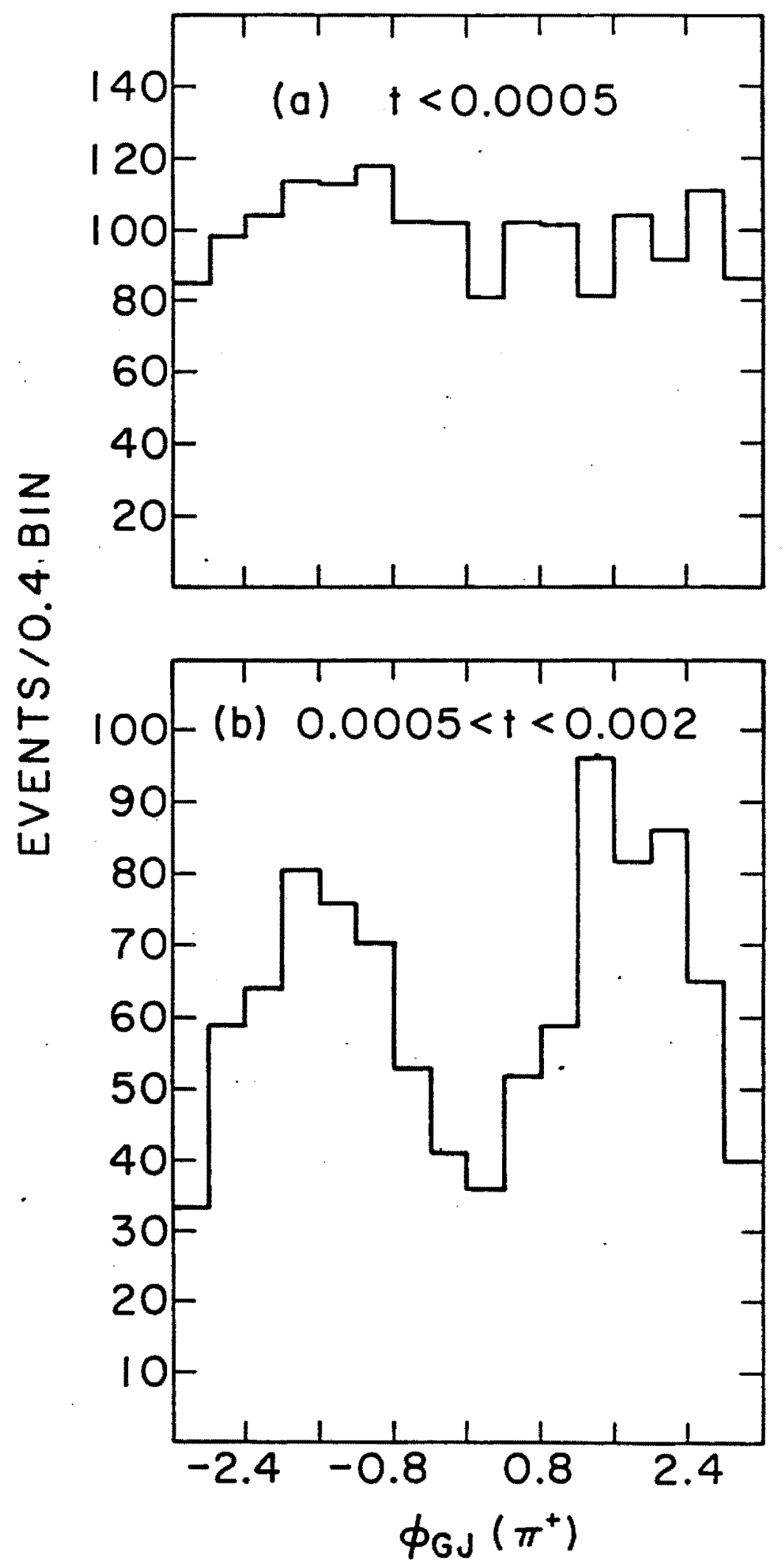

Figure 50 
to the $\cos \theta_{G J}$ distribution due to the presence of contributions from the $\pi$-exchange Deck diagram. [51] The good energy resolution obtained in our experiment enabled us to eliminate most of this type of background. The distribution of total reconstructed energy for $\rho^{+}$events is shown in Figure 51 a for the lead target. Cuts have been placed on interaction vertex, $\pi^{\circ}$ mass, and $\rho^{+}$mass, and the target empty contribution has been subtracted. The upper data have no restriction on $t$; the shaded region shows the effect of imposing a $t$ less than $.002 \mathrm{Gev}^{2}$ cut. Some inelastic background is evident in the low energy tail. Most of this background was eliminated by requiring the reconstructed energy to be in the range 192-212 GeV, shown by the two arrows in the figure. In Figure 51b, the shaded energy distribution above ( $t$ less than $.002 \mathrm{GeV}^{2}$ ) is compared to the Monte Carlo prediction.

The two photon mass distribution for $\rho^{+}$events on the lead target is shown in Figure 52a, along with the Monte Carlo prediction. The target empty contribution has been subtracted and the events have been restricted to $t$ less than $.002 \mathrm{Gev}^{2}$, in addition to the standard cuts described above. The good spatial and energy resolution in the LAC enabled us to achieve a very good mass resolution for the $\pi^{\circ}(\sigma=5 \mathrm{MeV})$. Candidates with 2 photon mass in the range shown by the two arrows $(.110-.160 \mathrm{GeV})$ were used in the analysis. It should 


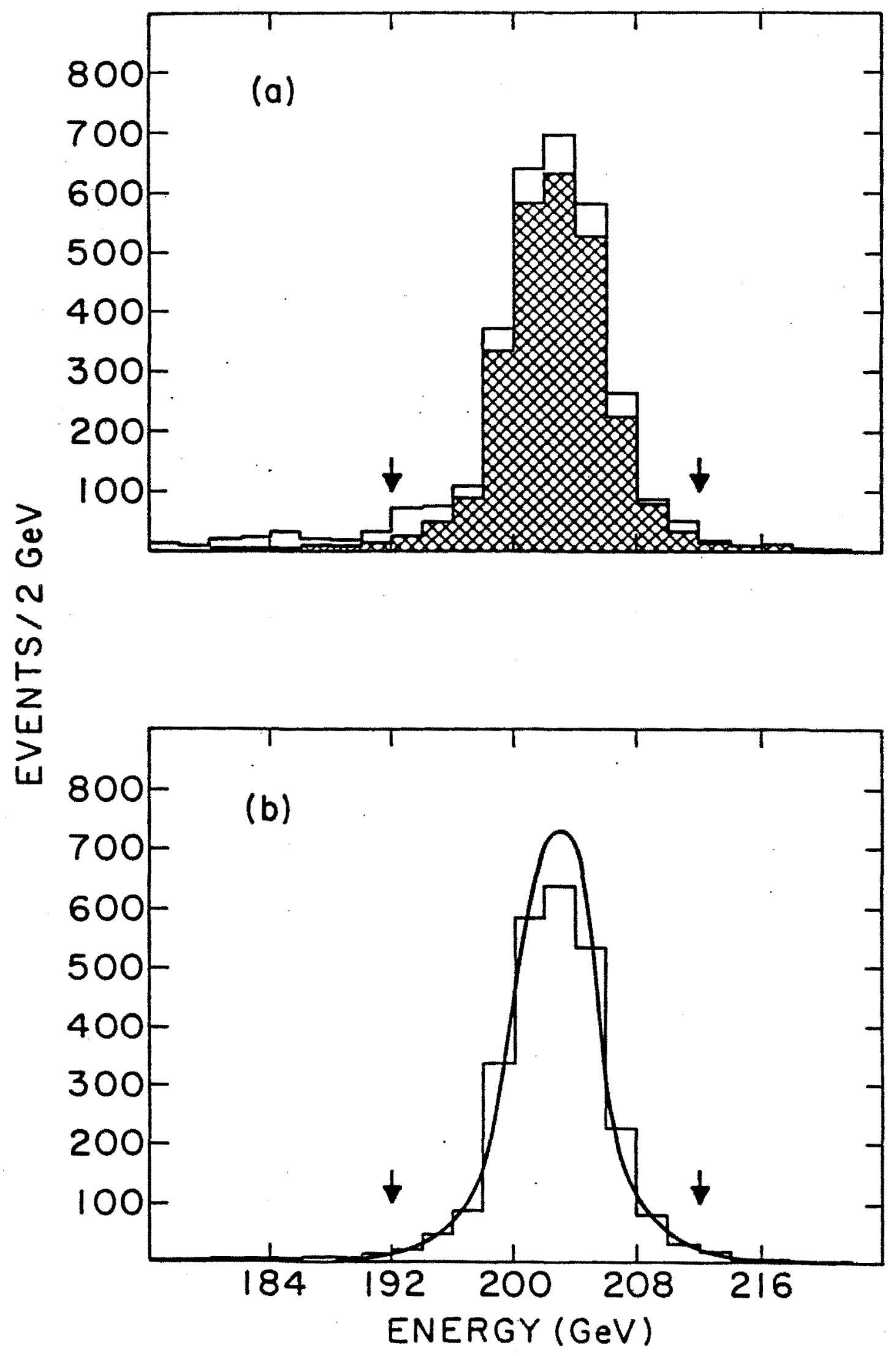

Figure 51 

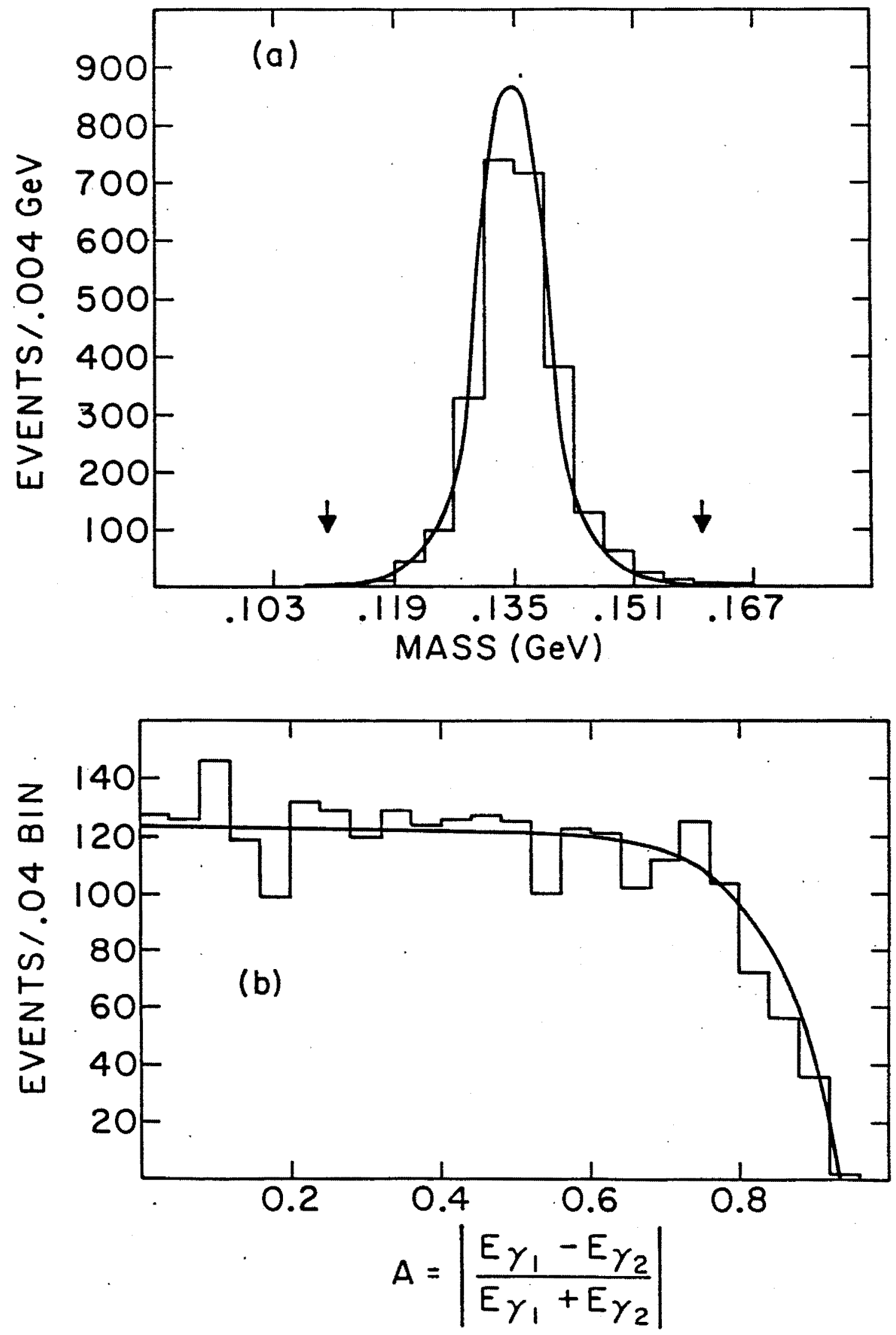

Figure 52 


\section{$-165-$}

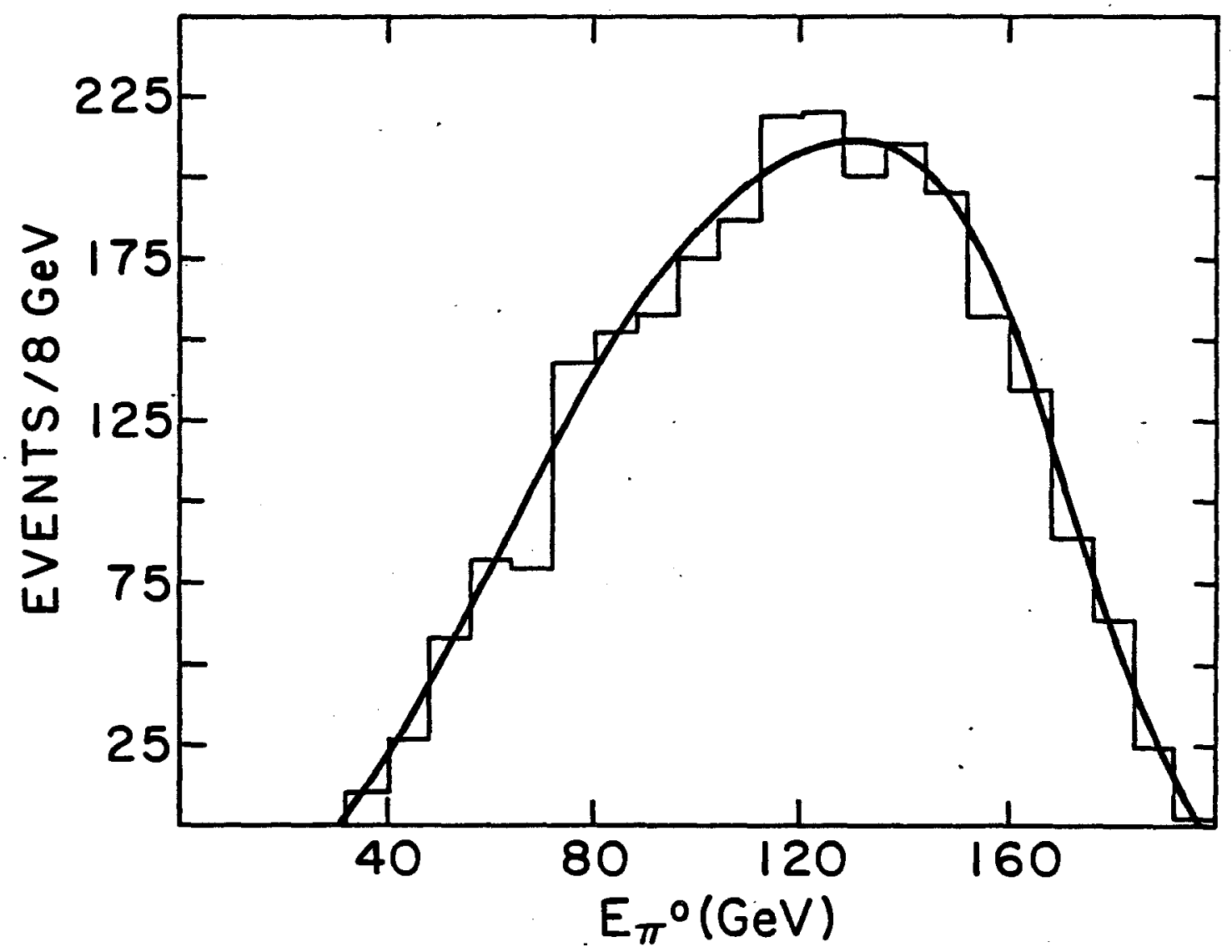

Figure 53 
be restated that the restrictions on the 2 photon mass and on the total reconstructed energy that were used in the $\rho^{+}$ analysis were the same as those used in the extraction of the $\mathrm{K}^{+} \rightarrow \pi^{+} \pi^{\circ}$ decay normalization factor.

In Figure $52 \mathrm{~b}$, the asymmetry distribution of the two photons is plotted for $\rho^{+}$events on the lead target. In addition to the restrictions outlined above, the events in the distribution also have the cut on $\pi^{\circ}$ mass. The agreement between Monte Carlo and the data indicates that the Monte Carlo correctly estimates the loss of photons.

The $\pi^{\circ}$ energy spectrum is shown in Figure 53 for $p^{+}$ events. The cuts applied are the'same as for the asymmetry distribution. The average $\pi^{\circ}$ energy in this distribution is 120 GeV.(For $\mathrm{K}^{+}$decays the average is $130 \mathrm{GeV.}$ ) The distribution is somewhat similar to that seen for $\mathbb{R}^{+} \rightarrow \pi^{+} \pi^{\circ}$ decays. (see Figure 38 ), although not quite as skewed towards higher energies.

\section{Mass Fittings}

The acceptance corrected $\pi^{+} \pi^{\circ}$ mass distributions are shown for the lead and copper targets in Figures 54 and 55. The cuts described in the last section on interaction vertex, total energy and $\pi^{\circ}$ mass have been applied. The 


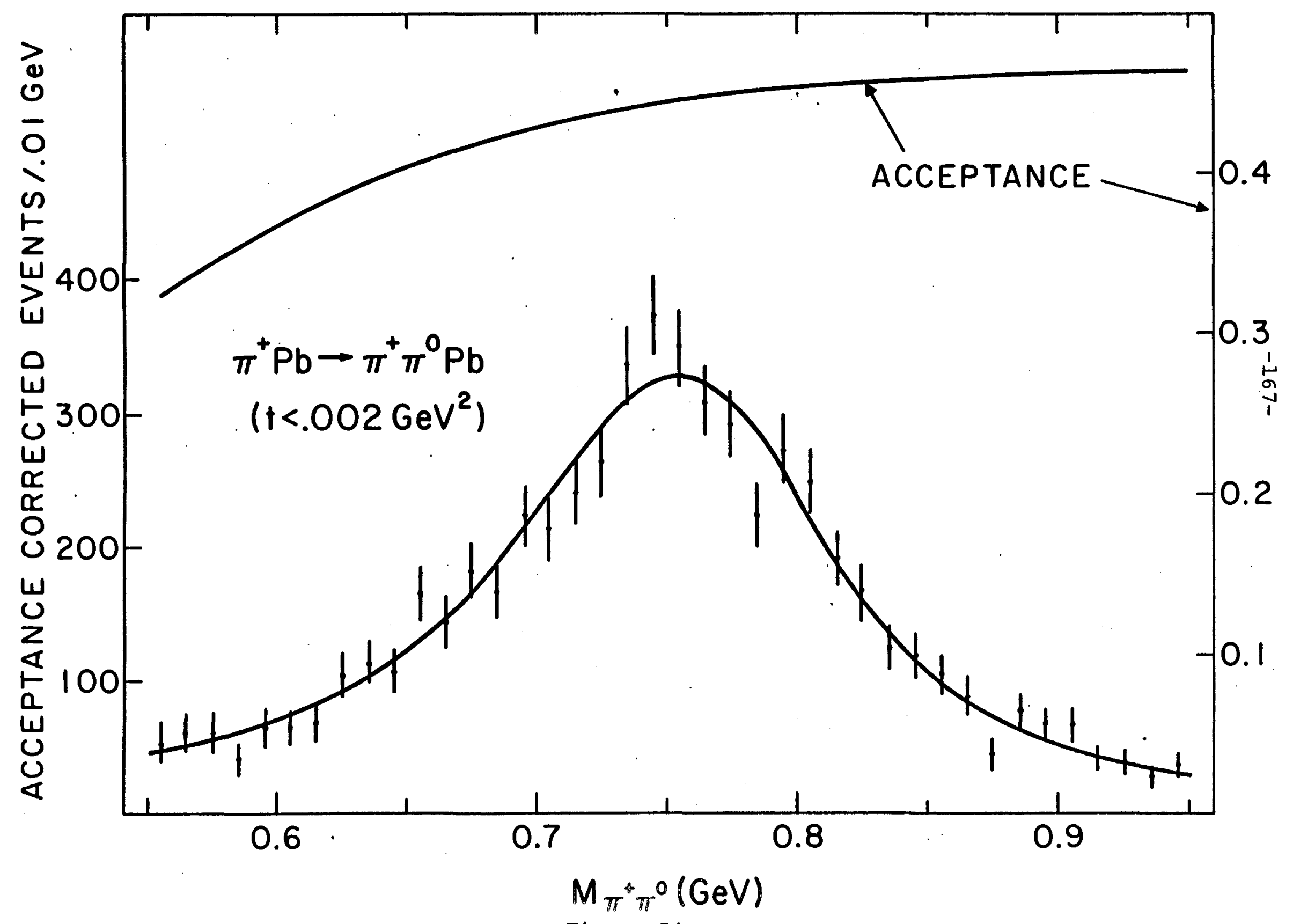

Figure 54 


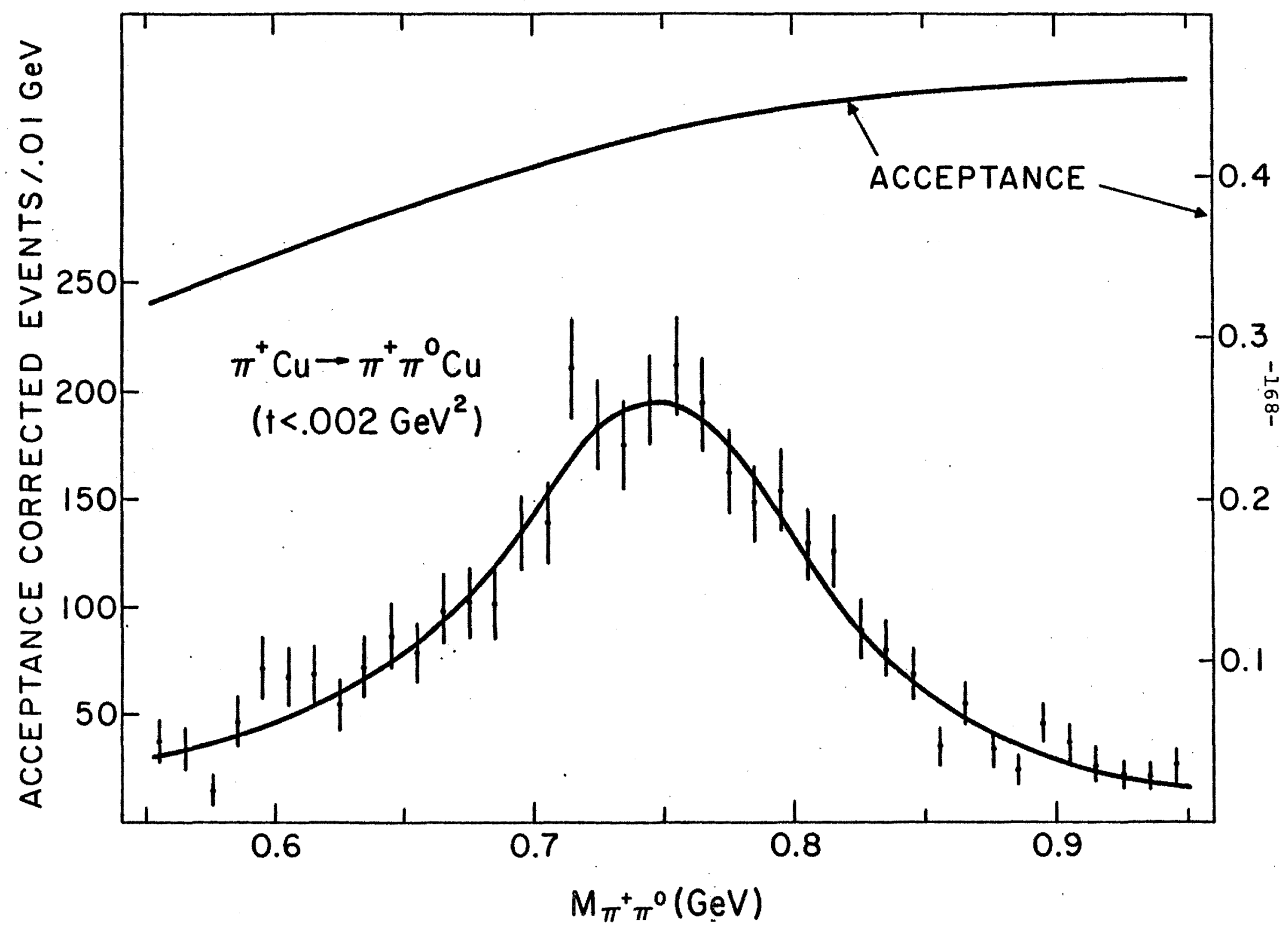


acceptance as a function of mass, shown above the mass distributions, includes the effects of these cuts as well as the reconstruction efficiency and the geometric acceptance. The events in the mass distribution, in addition, have been restricted to $t$ less than $.002 \mathrm{GeV}^{2}$ to enhance the coulomb production component.

Since the $\rho^{+}$is a resonance, the mass spectrum is described by a Breit-wigner formula. Some distortion from this simple formula is observed in our experiment, howèer, since Coulomb production has an intrinsic mass dependence. This can be seen from Equation (1.48) in Chapter I. Integrating Equation $(1.48)$ over $t$ (and substituting in the appropriate numbers for $\rho^{+}$production), we obtain an expression for the differential cross section with respect to the invariant mass squared.

$$
\begin{gathered}
\frac{d \sigma}{d m^{2}}=12 \pi \alpha z^{2} \frac{m^{2}}{\left(m^{2}-m_{\pi}{ }^{2}\right)^{3}} \Gamma_{\gamma}\left[\frac{1}{\pi} \frac{\Gamma_{\pi \pi^{\prime}}}{\left(m-m_{\rho}\right)^{2}+\left(\Gamma_{t o t} / 2\right)^{2}}\right] \\
\int_{t_{\min }}^{t_{\max } \frac{t-t_{\min }}{t^{2}}|F(t)|^{2} d t}
\end{gathered}
$$

Converting the expression in brackets to the relativistic form of the Breit-wigner(and letting $\Gamma_{\text {tot }}=\Gamma_{\pi \pi}$ ), and also converting the cross section to one with respect to the invariant mass, we obtain 


$$
\begin{aligned}
\frac{d \sigma}{d m}=48 \pi \alpha z^{2} & \frac{m^{3}}{\left(m^{2}-m_{\pi}^{2}\right)^{3}} \Gamma_{Y}\left[\frac{1}{\pi} \frac{m_{\rho}^{2} \Gamma_{\pi \pi}}{\left(m^{2}-m_{\rho}\right)^{2}+\left(m_{\rho} \Gamma_{\pi \pi}\right)^{2}}\right] \\
& \int_{t_{\min }}^{t_{\max }} \frac{t-t_{\min }}{t^{2}}|F(t)|^{2} d t
\end{aligned}
$$

The integral over $t$ is a function of the mass $m$ due to the mass dependence of $t_{\min }$. If we set $|F(t)|^{2}=e^{-b t}$ (where $b=400$ for lead and $b=200$ for copper) and allow tmax to go to infinity (integrating with a $t_{\max }$ of infinity instead of .002 results in an answer that differs by a simple multiplicative constant), the above integral can be approximated

$$
f(m)=\int_{\min }^{\infty} \frac{t-t_{\min }}{t^{2}} e^{-b t} d t \simeq-1.577-1 n\left(b t_{\min }\right)
$$

where, again, the mass dependence is contained in $t_{m i n}$.

Some additional distortion from the simple Breit-wigner shape occurs because of the mass dependence of the two widths $\Gamma\left(\rho^{+}+\pi^{+} \gamma\right)$ and $\Gamma\left(\rho^{+}+\pi^{+} \pi^{\circ}\right)$. If the two body decay of a resonance proceeds through a partial wave of orbital angular momentum $l$, the width for either of the two processes, with mass dependence included, can be written

$$
\Gamma(q)=\Gamma_{0}\left[\frac{q}{q_{0}}\right] 2 l+1 \frac{x(q)}{x\left(q_{0}\right)}
$$


where $q$ is the 3-momentum of each particle in the resonance rest frame and the subscript o refers to the value at the resonance mass. [52] $x(q)$ is an additional semi-phenomenological factor and for $\rho^{+}$production $\ell=1$. Table VI shows the different forms for $\Gamma\left(\rho^{+}+\pi^{+} \pi^{\circ}\right)$ and $\Gamma\left(\rho^{+}+\pi^{+} \gamma\right)$ used in the mass fittings. Note that some forms of $\Gamma\left(\rho^{+} \rightarrow \pi^{+} \gamma\right)$ used involves the square of the $\left(k / k_{0}\right)$ term instead of the cube. (To prevent confusion, I'll use $k$ to describe the decay particle 3 -momentum in the $\rho^{+} \rightarrow \pi^{+} \gamma$ case.) This was arrived at in analogy with the form of the width used in photoproduction experiments. [53]

Using the forms of the widths listed in Table VI, Equation (6.2) was used to fit the mass spectra for the lead and copper targets. The three parameters in the fit were the total width $\Gamma_{0}$, the resonance mass $m_{\odot}$, and an arbitrary normalization.

The results of the mass fittings are presented in Table VI. The best results are obtained with $\Gamma_{\gamma}=\Gamma_{O}(\gamma)$ $\left(k / k_{0}\right)^{2}\left(2 k_{0}{ }^{2}\right) /\left(k^{2}+k_{0}{ }^{2}\right)$ and $\Gamma_{\pi \pi}=\Gamma_{0}(\pi \pi) \quad\left(q / q_{0}\right)^{3}\left(2 q_{0}{ }^{2}\right) /\left(q^{2}+q_{0}{ }^{2}\right)$ or $\Gamma_{\pi \pi}=\Gamma_{O}(\pi \pi)\left(q / q_{0}\right)^{3} m_{0} / m$. Two other solutions have somewhat higher $x^{2}$ and the rest are prohibitively high. The two solutions with the best $x^{2}$ are compared to the data in Figures 54 and 55 (essentially they are described by the same curve). 


\section{Table VI}

Results of Mass Fits

Forms of Mass Dependence

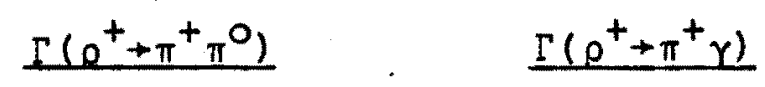

(1) $\left(\frac{\mathrm{q}}{\mathrm{q}_{0}}\right)^{3} \frac{\mathrm{m}}{\mathrm{m}}$

(1)

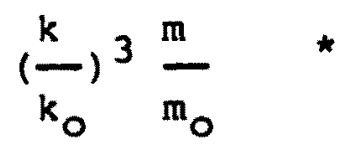

(2) $\left(\frac{q}{q_{0}}\right)^{3} \frac{2 q_{0}^{2}}{q^{2}+q_{0}^{2}}$

$\left(\frac{k}{k_{0}}\right)^{3} \frac{2 k_{o}^{2}}{k^{2}+k_{0}^{2}}$

(3)

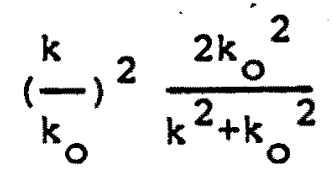

(4)

$\left(\frac{k}{k_{0}}\right)^{2} \frac{\mathrm{m}}{\mathrm{m}_{\mathrm{o}}}$

* For simplicity's sake I have switched from $q$ to $k$ in describing the different forms for $\Gamma\left(\rho^{+}+\pi^{+} \gamma\right)$.

Target Form of

Pb

$$
\Gamma_{\gamma} \Gamma_{\pi \pi} \text { Resonance Mass (GeV) }
$$

width (GeV)

$.1422 \pm .0046$

$.1430 \pm .0053$

$.1516 \pm .0060$

$.1516 \pm .0060$

$.1493 \pm .0057$

$.1492 \pm .0058$

$.1478 \pm .0059$

$.1480 \pm .0058$

$.1418 \pm .0072$

$.1427 \pm .0072$

$.1529 \pm .0078$

$.1528 \pm .0065$

$.1509 \pm .0075$

$.1507 \pm .0075$

$.1484 \pm .0076$

$.1486 \pm .0075$ $x^{2} /$ DOF

$97.2 / 37$

$97.8 / 37$

$45.8 / 37$

$44.9 / 37$

$41.2 / 37$

$41.4 / 37$

$63.4 / 37$

$60.8 / 37$

$80.2 / 37$

$77.0 / 37$

$45.9 / 37$

$45.2 / 37$

$40.8 / 37$

$40.8 / 37$

$58.2 / 37$

$56.5 / 37$ 
The values obtained for the total width of the $\rho^{+}$are very similar for the copper and lead targets for the two best solutions. The fitted values for the resonance mass differ, however, by $6 \mathrm{MeV}$, about 2-3 standard deviations. This is a measure of the systematic error in the determination of the resonance mass. If we take the statistical error weighted means of the resonance mass and of the total width obtained for the two targets, and add the systematic and statistical errors quadratically to get the total error, we obtain

$$
\begin{aligned}
& m_{0}=0.7697 \pm 0.0035 \mathrm{GeV} \\
& I_{0}=0.1497 \pm 0.0048 \mathrm{GeV}
\end{aligned}
$$

Again, these values are derived from the two solutions with the lowest $x^{2}$. These results agree well with those listed in the Particle Data Book. [54] It should be noted that our determination of the mass and width of the $\rho^{+}$meson might be one of the cleanest since the production mechanism is well understood. Previous attempts to fit the charged $\rho$ mass and width have used either OPE (One Pion Exchange) models [55] or simply phase space [56] to describe the production mechanism.

For simplicity's sake, no strong contributions have been considered in the mass fittings. strongly produced $\rho^{+}$ mesons have a slightly different mass shape since no coulomb distortion is present in the production mechanism. However, as will be seen later, for $t$ values of less than $.002 \mathrm{GeV}^{2}$ the production is overwhelmingly Coulomb. 
D. Calculation of Cross Sections

The cross section per event can be written

$$
\begin{aligned}
\sigma(\text { per event })= & \left(A / N_{\circ} \rho t\right) /(\text { Normalized beam } f l u x) \\
& \times \text { Geometric and reconstruction losses } \\
& \times \text { Absorption losses } \\
& \times \mathrm{K}^{+}+\pi^{+} \pi^{\circ} \text { normalization factor }
\end{aligned}
$$

$A$ is the atomic weight of the target nucleus, $N_{0}$ is Avogadro's number and $\rho$ is the thickness of the target in grams $/ \mathrm{cm}^{2}$. The incident beam flux was defined as outlined in Chapter II and normalized using the correction factors discussed in Chapter IV (Tables IIa and IIb). The $\rho^{+}$cross section was corrected for geometric and reconstruction losses, initial and final state absorption, and finally the $\mathrm{K}^{+} \rightarrow \pi^{+} \pi^{\circ}$ decay rate normalization. The last two corrections were discussed in some detail in Chapter $V$ and are summarized in Tables IV and $V$. The first correction, from geometric and reconstruction losses, was estimated using the Monte Carlo and reconstruction programs as discussed earlier in this chapter. Note that reconstruction losses include the effect of the various cuts used in the analysis. The correction factors discussed above are summarized and the cross section per event is given for each of the three targets in Table VII. 
Table VII

Summary of Correction Factors for $\rho^{+}$production

\section{Target}

$\underline{\mathrm{C}} \quad \underline{\mathrm{Cu}} \quad \underline{\mathrm{Pb}}$

\begin{tabular}{|c|c|c|c|}
\hline $\begin{array}{l}\text { Absorption } \\
\text { corrections }\end{array}$ & $1.372 \pm .008$ & $1.737 \pm .009$ & $1.43 .7 \pm .008$ \\
\hline $\begin{array}{l}\text { Geometric and } \\
\text { reconstruction } \\
\text { losses }\end{array}$ & $2.32 \pm .026$ & $2.326 \pm .021$ & $2.30 \pm .02$ \\
\hline $\begin{array}{l}\text { Interaction } \\
\text { vertex } \\
\text { correction }\end{array}$ & $0.92 \pm .02$ & $0.92 \pm .02$ & $0.92 \pm .02$ \\
\hline $\begin{array}{l}\mathrm{K}^{+} \rightarrow \pi^{+} \pi^{\circ} \\
\text { normalization }\end{array}$ & $1.16 \pm .03$ & $1.13 \pm .02$ & $1.14 \pm .02$ \\
\hline $\begin{array}{l}\text { Product of } \\
\text { above } 4 \\
\text { factors }\end{array}$ & $3.40 \pm .123$ & $4.20 \pm .125$ & $3.47 \pm .100$ \\
\hline $\begin{array}{l}\text { Normalization } \\
\text { of } \pi^{+} \text {beam }\end{array}$ & $0.648 \pm .012$ & $0.685 \pm .013$ & $0.682 \pm .013$ \\
\hline$\sigma(\mu b /$ event $)$ & $.077 \pm .003$ & $.077 \pm .0027$ & $.330 \pm .011$ \\
\hline
\end{tabular}


E. Extraction of the Radiative Width

The differential cross sections $(d \sigma / d t)$ for the three nuclear targets are shown in Figure 56. The cross sections were calculated as discussed in section $c$. The data were subjected to the cuts on interaction vertex, 2 photon mass, and total energy that were discussed previously. Also, to eliminate background from $\mathrm{K}^{+} \rightarrow \pi^{+} \pi^{\circ}$ decays, only events with $\pi^{+} \pi^{\circ}$ mass in the range .550-.950 GeV were used in the analysis. The correction for this mass cut will be discussed later in this section.

The $t$ distribution shown in Figure 56 displays the characteristics described in chapter $I$ for coherent $\rho^{+}$ production. Coulomb production dominates at low $t$, as seen by the sharp forward peak which increases roughly as $\mathrm{z}^{2}$ for the three nuclear targets. The tails to the $t$ distributions at large $t$ indicate the presence of strong $\rho^{+}$production. The solid lines in the figure indicate the results of fits of the data to Equation (1.54). The fitting formalism will now be discussed.

By making the narrow width approximation, we can write the total cross section in the form

$$
\frac{d \sigma}{d t}=\left|T_{c}+e^{i \phi_{T_{s}}}\right|^{2}
$$




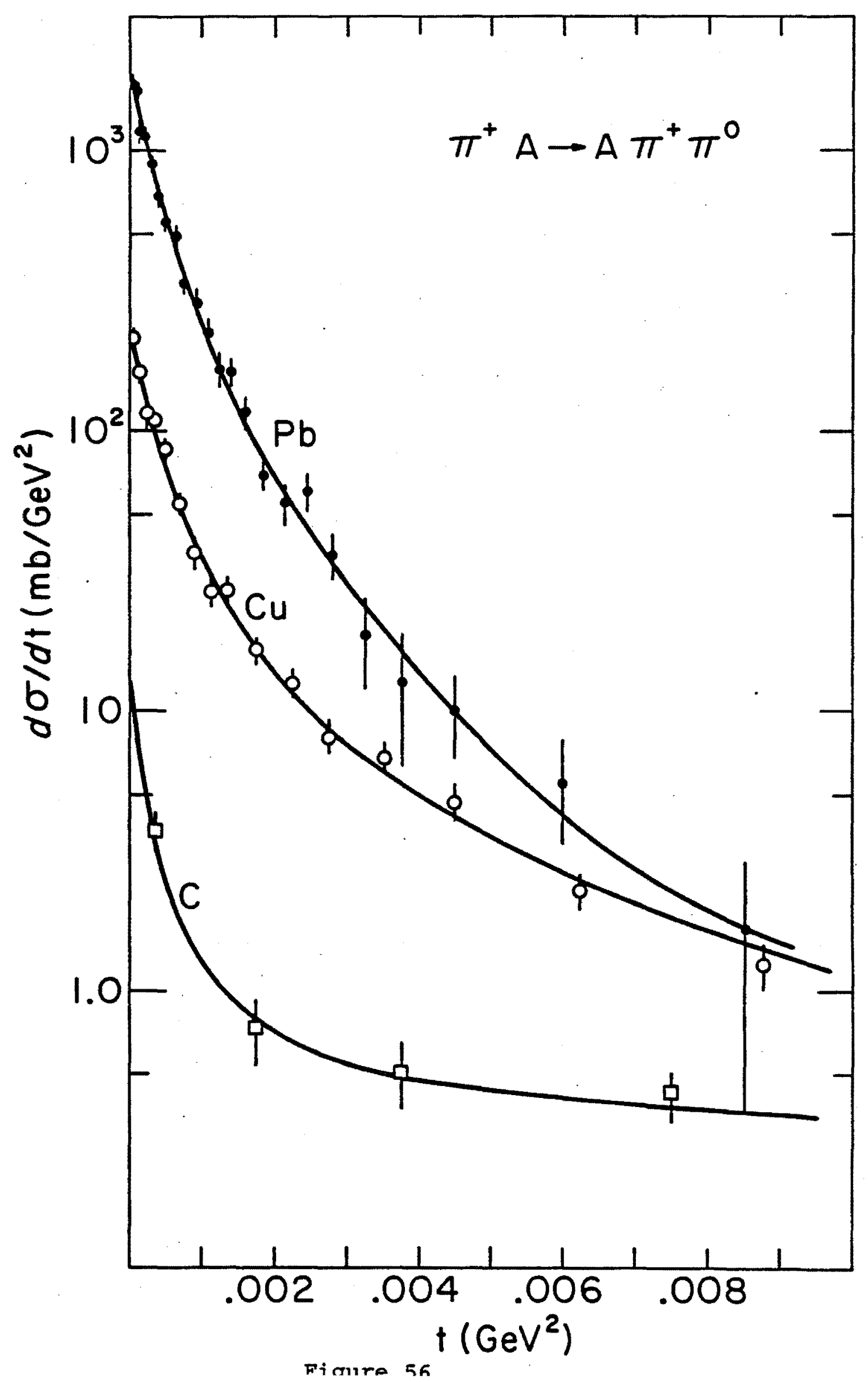


where $T_{c}$ and $T_{s}$ indicate the Coulombic and strong production amplitudes and $\phi$ is the relative phase between them. The Coloumbic amplitude can be written explicitly as

$$
\begin{aligned}
& T_{C}(q)=\left[\Gamma_{0}\left(\rho^{+}+\pi^{+} \gamma\right)\right]^{1 / 2} F_{C O u l}(q) \\
& F_{c o u l}(q)=\frac{z}{q^{2}}\left[24 \pi \alpha \frac{m_{\rho}^{3}}{\left(m_{\rho}{ }^{2}-m_{\pi}{ }^{2}\right)^{3}}\right]^{1 / 2} \sqrt{2}(\hat{\varepsilon} \times \vec{k}) F_{c}(q)
\end{aligned}
$$

where $F_{C}(q)$ is the form factor of the nucleus. The model of C. Bemporad et al [57] has been adopted to evaluate the form factor. The basics of this model will be sketched here. More details are available in. References 57 and 58.

In this model, the form factor $F_{c}(q)$ is divided into three regions, as shown in Figure 57. The form factor can be written

$$
F_{c}(q)=F_{c}{ }^{i n t}(q)+F_{c} e^{\operatorname{ext} 1_{+}} F_{c} \operatorname{ext2}^{\ln }
$$

Each term can be expressed

$$
\begin{aligned}
& F_{C}^{i n t}(q)=\frac{i q^{2}}{4 \pi p R^{3}} \int a^{3} r e^{-1 / 2 \sigma^{\prime} T(b)} e^{i X_{C}(b)} e^{i \vec{q} \cdot \vec{r}} \cdot \vec{r} \\
& F_{C} \operatorname{extl}(q)=\frac{i q^{2}}{4 \pi p} \int d^{3} r e^{i X_{C}(b) e^{i \vec{q} \cdot \vec{r}} \frac{\vec{r}}{r^{3}}}
\end{aligned}
$$




\section{$-179-$}

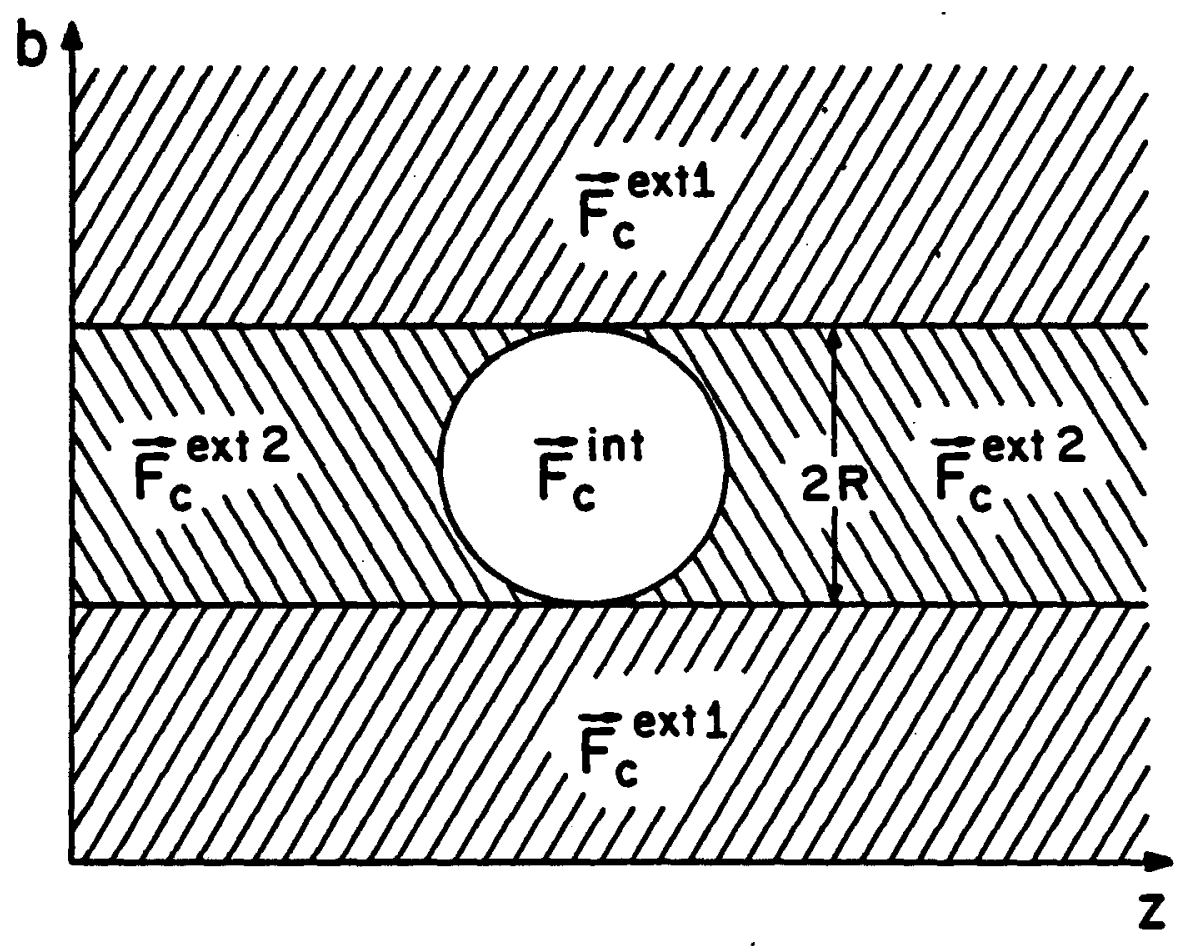

Figure 57 


$$
F_{C} \operatorname{eri}^{2}(q)=\frac{i q^{2}}{4 \pi p} \int a^{3} r e^{-1 / 2 \sigma^{\prime} T(b)} e^{i X_{c}(b)} e^{i \vec{q} \cdot \vec{r}} \frac{\vec{r}}{r^{3}}
$$

The form factor not only takes into account the nuclear shape but also the absorption of the incoming and outgoing states within the nucleus and the coulomb phase added due to the nuclear charge distribution. The definition of some of the terms used in the form factor are provided below:

1. $\sigma^{\prime}=\sigma(1-i \alpha)$ where $\sigma$ is the total $\pi^{+}$(or $\rho^{+}$) nucleon cross section and $\alpha$ is the ratio of the real to the imaginary part of the forward scattering amplitude of $\pi^{+} \cdot s\left(\right.$ or $\left.\rho^{+} ' s\right)$ on nucleons. For this analysis, we have assumed that the incident $\pi^{+}$and outgoing $\rho^{+}$ have the same values of $\sigma$ and $\alpha$. [59]

2. $T(b)=A \int_{-\infty}^{+\infty} d z \rho(b, z)$ is the nuclear thickness defined in terms of the impact parameter $b . p(b, z)$ is the density distribution of nuclear matter.

3. $x_{c}(b)=-z e^{2} / h c \int_{-\infty}^{+\infty} d z \phi\left(\left[b^{2}+z^{2}\right]^{1 / 2}\right)$ is the coulomb phase due to the nuclear matter distribution.

$\phi=\int a^{3} r^{\prime} g(\vec{r}) /\left(\left|\vec{r}-\vec{r}^{\prime}\right|\right)$ is the coulomb potential of the nucleus. 
Most Coulomb production takes place outside the nucleus. (See Chapter I) Therefore the form used for the nuclear density distribution will not seriously affect the calculation. For calculating the coulomb form factor above, we have assumed a nucleus with a uniform distribution and a radius of $R=\left(c^{2}+(7 / 3)\left(\pi a^{2}\right)\right)^{1 / 2}$ where $c=1.12 A^{1 / 3}$ fermi and $a=0.545$ fermi. $R$ is the equivalent radius of the woods-Saxon distribution of nuclear matter which was used in the form factor calculation for strong production, as will be discussed below.

The amplitude for coherent strong production can be written

$$
\begin{aligned}
& T_{s}(q)=\left[C_{s}\right]^{1 / 2} F_{s}(q) \\
& F_{s}(q)=A \int d^{3} r e^{i \vec{q} \cdot \vec{r}} e^{-1 / 2 \sigma ́ T(b)} e^{i \chi_{c}(b) \frac{\sqrt{2}(\hat{\varepsilon} \mathbf{x})}{q_{T}}} \nabla_{b} \rho(b, z)
\end{aligned}
$$

$C_{s}$ is a normalization constant and the other parameters have been described before. Because of the $\nabla_{b}(b, z)$ term above, strong production is sensitive to the nuclear density distribution. The dominant contribution to the strong cross section, in fact, comes from the region near the nuclear surface. (This is the origin of the effective $A^{2 / 3}$ 
dependence for the strong cross section discussed in Chapter I.) Because of this sensitivity to the nuclear matter distribution, an explicit woods-Saxon density distribution $\rho(r)=\rho_{o} /(1+\exp (r-c / a))$ was used where $c$ and a have been defined before.

The differential cross section, Equation $(6.5)$, can be decomposed, for computational purposes, into the following form

$$
\begin{aligned}
& \frac{d \sigma}{d t}=r_{0}\left(\rho^{+}+\pi^{+} \gamma\right)\left|F_{\operatorname{coul}}(q)\right|^{2}+c_{s}\left|F_{s}(q)\right|^{2} \\
& +2\left[\Gamma_{0}\left(\rho^{+} \rightarrow \pi^{+} \gamma\right) C_{s}\right]^{1 / 2} \quad x \\
& {\left[\left(F_{\text {coul }}{ }^{\operatorname{real}}(q) F_{S}{ }^{r e a l}(q)+F_{c o u l}{ }^{i m a g}(q) F_{s}{ }^{i m a g}(q)\right) \cos \phi\right.} \\
& +\left(F_{\text {coul }}{ }^{\left.\left.\operatorname{imag}(q) F_{s}{ }^{r e a l}(q)-F_{c o u l}{ }^{r e a l}(q) F_{s}{ }^{i m a g}(q)\right) \sin \phi\right]}\right.
\end{aligned}
$$

The four terms

$$
\begin{array}{ll}
\left|F_{\text {coul }}(q)\right|^{2} & (6.13 a) \\
\left|F_{S}(q)\right|^{2} & (6.13 b) \\
\left(F_{c o u l}{ }^{r e a l}(q) F_{s}{ }^{r e a l}(q)+F_{c o u l}{ }^{i m a g}(q) F_{s}{ }^{i m a g}(q)\right) & (6.13 c) \\
\left(F_{c o u l}{ }^{i m a g}(q) F_{s}{ }^{r e a l}(q)-F_{c o u l}{ }^{r e a l}(q) F_{s}{ }^{i m a g}(q)\right) & (6.13 d)
\end{array}
$$


were calculated over the $t$ range of interest, then corrected for the mass cut imposed on the $\rho^{+}$analysis and for the narrow width approximation that were made before. These corrections will be discussed in more detail below.

To simplify the form of the Coulombic differential cross section, Equation (1.49), the assumption was made that $\Gamma_{0}\left(\right.$ total width) $<m_{0}$. By making this assumption, the Breit-wigner term in Equation (1.49) simplifies to a delta function, making the integration over mass trivial. The simplified form of the Coulomb differential cross section is shown in Equation (1.51). However, the total width of the $\rho^{+}$ meson (150 Mev) is comparable to the resonance mass $(770 \mathrm{MeV})$. The explicit mass dependence in Equation (1.49) (and the implicit mass dependence contained in $t_{\text {min }}$ ) make it necessary for a correction to be applied to the terms (above) calculated with the narrow width approximation. The ratio of Equation (1.49) to Equation (1.51) can be written

Ratio=Coulombic correction term

$$
=\frac{1}{2} \int\left(\frac{m_{\rho}^{2}-m_{\pi}^{2}}{m^{2}-m_{\pi}^{2}}\right)^{3} \frac{m^{2}}{m_{\rho}^{3}} \frac{t-t_{\min }(m)}{t-t_{\min }\left(m_{\rho}\right)} g_{\rho}(m)\left[\frac{1}{\pi} \frac{r / 2}{\left(m-m_{\rho}\right)^{2}+(\Gamma / 2)^{2}}\right] d m^{2}
$$


or changing the form of the Breit-wigner

$$
\begin{aligned}
& \text { Ratio }= \\
& \qquad\left(\frac{m_{\rho}^{2}-m_{\pi}^{2}}{m^{2}-m_{\pi}^{2}}\right) \frac{m^{2}}{m_{\rho}^{2}} \frac{t-t_{m i n}(m)}{t-t_{m i n}\left(m_{\rho}\right)} g_{\rho}(m)\left[\frac{I}{\pi} \frac{m_{\rho} \Gamma}{\left(m^{2}-m_{\rho}\right)^{2}+\left(m_{\rho} \Gamma\right)^{2}}\right] d m^{2}
\end{aligned}
$$

Here, $\Gamma$ is the total width of the $\rho^{+}$and $g_{\rho}(m)$ contains the mass dependence of the radiative width. (The mass dependence of the two widths was discussed in section B.) For the above formulas, the mass was taken to be . $770 \mathrm{GeV}$ and the total width was taken to be $.150 \mathrm{GeV}$.

To correct for the mass cut imposed in the analysis, the integration above was only taken between these two limits: $(.550 \mathrm{GeV})^{2}$ to $(.950 \mathrm{Gev})^{2}$. The mass dependences of the two widths were those most successful in fitting the line shape of the $\rho^{+}:\left(q / q_{0}\right)^{3} 2 q_{0}{ }^{2} /\left(q^{2}+q_{0}{ }^{2}\right)$ for the total width and $\left(k / k_{0}\right)^{2} 2 k_{0}{ }^{2} /\left(k^{2}+k_{0}{ }^{2}\right)$ for the radiative width.

The interference terms are only partially coulombic so they were corrected by 
Interference correction term=

$$
\begin{gathered}
\int\left[\left(\frac{m_{\rho}^{2}-m_{\pi}^{2}}{m^{2}-m_{\pi}^{2}}\right) \frac{m^{2}}{m_{\rho}^{2}}\right]^{1 / 2} g_{\rho}(m)\left[\frac{t-t_{m i n}(m)}{t-t_{m i n}\left(m_{\rho}\right)}\right]^{1 / 2} \\
{\left[-\frac{m_{\rho} \Gamma}{\left.\pi m^{2}-m_{\rho}{ }^{2}\right)^{2}+\left(m_{\rho} \Gamma\right)^{2}}\right] d^{2}}
\end{gathered}
$$

and since the strong term contains no explicit mass dependence, it was corrected only for the loss of events due to the mass cut.

$$
\begin{aligned}
& \text { strong correction term= } \\
& \int g_{\rho}(m)\left[\frac{1}{-\pi} \frac{m_{\rho} \Gamma}{\left(m^{2}-m_{\rho}{ }^{2}\right)^{2}+\left(m_{\rho} \Gamma\right)^{2}}\right] d m^{2}
\end{aligned}
$$

Before comparing to the data, the calculated cross section, Equation (6.12), had to be corrected for the $t$ resolution of the spectrometer. The observed cross section $\mathrm{d} \sigma / \mathrm{dt}$ is a convolution of the true production cross section $d \sigma / a \tilde{t}$ with the resolution function $R(t, \tilde{t})$ :

$$
\frac{d \sigma}{d t}=\int d \tilde{t} R(t, \tilde{t}) \frac{d \sigma}{d \tilde{t}}
$$


We have assumed that the resolution function can be parameterized as a two dimensional gaussian in transverse momentum

$$
R(t, \tilde{t})=\frac{1}{2 \pi \sigma^{2}} e^{-\left(\left|\vec{q}_{T}-\tilde{\vec{q}}_{T}\right|\right)^{2} / 2 \sigma^{2}}
$$

where $\tilde{\vec{q}}_{T}$ and $\vec{q}_{T}$ are the exact and observed transverse momentum, respectively, and $\sigma$ is the standard deviation of the resolution function. This hypothesis was tested with $\mathrm{K}^{+}+\pi^{+} \pi^{\circ}$ decays and found to work.well. (See Chapter IV and Appendix B.) The resolution, $\sigma$, used for each target was the quadratic sum of the $t$ resolution found in the Monte Carlo for $\rho^{+}$production and a residual term determined by comparing the Monte Carlo and data resolutions for the process $\mathrm{K}^{+}+\pi^{+} \pi^{\circ}$. The Monte Carlo and residual terms are shown in Table VIII for each target along with the quadratic sum. Folding in the $t$ resolution, the $t$ dependent factors 16.13 $a-d)$ were calculated in $t$ intervals for comparison to the data. The parameters $\Gamma_{0}\left(\rho^{+}+\pi^{+} Y\right) ; C_{S}$, and $\phi$ were varied to obtain the best fit.

The results of the fits to the data, for the $t$ ranges indicated, are shown in Table IX. For the global fit, $C_{S}$ and $\phi$ were assumed to be independent of target material. The errors on the parameters are purely statistical. Some 
Table VIII

Resolutions for $\rho^{+} t$ Fittings

\begin{tabular}{|c|c|c|c|c|}
\hline Target & $\sigma_{\text {Monte }}$ & $\operatorname{Car} 10(\mathrm{MeV})$ & $\sigma_{\text {Residual }}(\mathrm{MeV})$ & {$\left[\sigma_{M C}{ }^{2}+\sigma_{\operatorname{Res}}{ }^{2}\right]^{1 / 2}$} \\
\hline c & & 9.0 & 3.5 & 9.6 \\
\hline $\mathrm{Cu}$ & & 12.0 & 4.8 & 12.9 \\
\hline $\mathrm{Pb}$ & & 10.1 & 5.2 & 11.4 \\
\hline
\end{tabular}


Table IX

Results of Fits to $t$ Distributions

\begin{tabular}{ccccccc} 
Target $\begin{array}{c}t \text { range } \\
\left(\mathrm{GeV}^{2}\right)\end{array}$ & $\begin{array}{c}\Gamma\left(\rho^{+}+\pi^{+} \gamma\right) \\
(\mathrm{KeV})\end{array}$ & $\begin{array}{c}C_{\mathrm{s}} \\
\left(\mathrm{mb} / \mathrm{GeV}^{2}\right)\end{array}$ & $\begin{array}{c}\phi \\
\text { (degrees) }\end{array}$ & $x^{2} / \mathrm{DOF}$ \\
\cline { 1 - 1 } $\mathrm{C}$ & $<.04$ & $47.30 \pm 6.95$ & $0.54 \pm .07$ & $0.0 \pm 92.8$ & $0.7 / 4$ \\
$\mathrm{Cu}$ & $<.01$ & $59.49 \pm 1.88$ & $0.24 \pm .08$ & $15.30 \pm 39.60$ & $14.2 / 13$ \\
$\mathrm{~Pb}$ & $<.01$ & $59.30 \pm 1.64$ & $1.43 \pm .55$ & $86.75 \pm 10.98$ & $13.5 / 20$ \\
Global & $<.01$ & $59.78 \pm 1.15$ & $0.34 \pm .13$ & $41.26 \pm 23.62$ & $35.9 / 40$
\end{tabular}


Table $\mathrm{X}$

Integrated cross sections for $\rho^{+}$Production

$\begin{array}{cccc}\text { Target } & \begin{array}{c}\int d t\left|F_{C}\right|^{2} \\ (\mu b)\end{array} & \begin{array}{c}\int d t\left|F_{S}\right|^{2} \\ (\mu b)\end{array} & \begin{array}{c}2 \operatorname{Re} \int d t F_{C}^{*} F_{S} e^{i \phi} \\ (\mu b)\end{array} \\ & 4.7 \pm .69 & 1.23 \pm .16 & 2.13 \pm .28 \\ \mathrm{Cu} & 122.9 \pm 3.9 & 5.11 \pm 1.70 & 18.2 \pm 2.8 \\ \mathrm{~Pb} & 848.0 \pm 22.6 & 69.9 \pm 26.9 & 25.3 \pm 26.9\end{array}$


systematic effects were examined to check the stability of the solutions. Changing the $t$ range of the fit results in a change of $\Gamma_{0}\left(\rho^{+} \rightarrow \pi^{+} \gamma\right)$ of the order of $\pm 1-28$, within the statistical error. As mentioned previously, the LAC position was determined by two techniques: one using information from $\pi$-e scattering events and Ke 3 decays, and the other using $\mathrm{K}^{+} \rightarrow \pi^{+} \pi^{\circ}$ events and moving the LAC in order to minimize the observed resolution in $t$. The LAC position determined from the latter method was used in the analysis. (They differed by $30 \mathrm{mils}$ in $X$ and $8 \mathrm{mils}$ in $Y$.$) To check for any bias; the$ analysis was repeated, for data on the lead target, using the LAC position determined by the first method. Less than a 18 change in the value of $\Gamma$ was seen. A rather severe cut on the interaction vertex was used in order to cut down on the target empty background. using a more liberal cut on the interaction vertex $(-35 \mathrm{~cm}+25 \mathrm{~cm})$, the analysis was repeated for the lead data and the change in the value of the radiative width was found to be 1.58 . The line shape of the $\rho^{+}$meson was used in the $t$ fits to correct for events on the tails lost due to the mass cut(.550-950 GeV). Varying the values of the resonance mass and of the total width by \pm one sigma(from the results listed in section C) results in changes of the value of $\Gamma_{0}\left(\rho^{+} \rightarrow \pi^{+} \gamma\right)$ of \pm 1.58 and \pm 1.08 , respectively. 
In the $\rho^{+}$analysis, a residual resolution term, determined from $\mathrm{K}^{+} \rightarrow \pi^{+} \pi^{\circ}$ decays, was added quadratically to the $t$ resolution determined from the $\rho^{+}$Monte Carlo. The resulting resolution was used in the $t$ fits of the $\rho^{+}$data. As a test of the propriety of this measure, the data was in addition fit using other values for the $t$ resolution, both larger and smaller than the quadratic sum of the Monte carlo and residual terms. Increasing $\sigma_{p_{t}}{ }^{2}$ by $10 \%$ for both the copper and lead targets results in an increase of the radiative width of approximately 18. For 38 degrees of freedom, it also increase the total $x^{2}$ of the fit by 3 . Decreasing $\sigma_{p_{t}} 2$ by 108 results in a decrease in the radiative width of about 18 but, again for 38 degrees of freedom, causes an increase in the total $x^{2}$ of about 4 . Using the Monte Carlo derived values for $\sigma_{p_{t}} 2$ results in a decrease in the fitted value of $\Gamma\left(\rho^{+}+\pi^{+} \gamma\right)$ of about 2.58 but an increase in the total $\chi^{2}$ of about 23. The fact that the value chosen in the analysis for $\sigma_{p_{t}} 2$ gives the best value of $x^{2}$ lends confidence to the procedure used to arrive at it. The error in the radiative width due to the uncertainty in $\sigma_{p_{t}} 2$ is estimated to be 18 .

In Table $X$ are presented the integrated values of $\left|F_{c}\right|^{2},\left|F_{s}\right|^{2}$ and the interference term for $t$ of less than .01 $\mathrm{GeV}^{2}$. The values are obtained from integrations over the fitted curves. 
F. Conclusions

Based on the global fit of the three targets presented in the last section, we quote a new value for the radiative width,$\Gamma_{0}\left(\rho^{+}+\pi^{+} \gamma\right)=60 \pm 4$ ReV. The error is a quadratic sum of the statistical error of the fit and of the total systematic error. Some of the major sources of systematic error are: $K^{+}$decay normalization( \pm 28$)$, muon contamination( $( \pm 1.58)$, and interaction vertex correction $( \pm 28)$. The values for $C_{s}$ and $\phi$ are not very well determined since Coulomb production dominates, but are roughly consistent with extrapolations made from measurements at lower energies. [60]

A previous measurement of our group [61], of the radiative width of the $\rho^{-}$, obtained a value, $\Gamma_{0}\left(\rho^{-} \rightarrow \pi^{-} \gamma\right)=71 \pm 7$ Kev. This value is somewhat higher than that quoted in this thesis (but consistent at about the 208 level of probability). The previous measurement was conducted at two beam energies, $156 \mathrm{GeV}$ and $260 \mathrm{GeV}$, with the bulk of the data being taken at $156 \mathrm{GeV}$. Six targets were used: carbon, aluminum and two thicknesses each of lead and copper. The value of $71 \mathrm{kev}$ was obtained from a weighted average of the global fits at the two different energies. 
The spectrometer used was the same for the two measurements. For the measurement quoted in this thesis, however, certain modifications were made that led to an improvement in the spectrometer resolution. In addition, the data sample (of $\rho$ events) was about two times larger for this measurement than for the previous one. The systematic error was also somewhat lower, leading to a smaller total error. If we consider the two quoted values, $60 \mathrm{KeV}$ and $71 \mathrm{KeV}$, as independent measurements(assuming there is no significant production difference due to the different charges), and calculate the error weighted mean, we obtain: $\Gamma_{0}(\rho+\pi Y)=63 \pm 4 \mathrm{ReV}$. This value is in reasonable agreement with that expected from the $\nabla D M, S O(3)$, and non-relativistic quark model arguments discussed in Chapter I. 
FOOTNOTES

1. M. Gell-Mann and F. Zachariasen, Phys. Rev. 124 $.953(1961) ;$ Y. Nambu and J.J. Sakurai, Phys. Rev. Lett. $\underline{8}, 79(1962) ;$ M. Gell-Mann, D. Sharp, and W.G. Wagner, Phys. Rev. Lett. $8,261(1962) ;$ R.F. Dashen and D.H. Sharp, Physical Review 133,1585B(1964); N.M. Kroll, T.D. Lee, and B. Zumino, Phys. Rev. 157 , $1376(1967)$. For an excellent review see also, $R$. Feynman, Photon-Hadron Interactions , (W.A. Benjamin, Inc., Reading, Mass. 1972).

2. J.J.. Sakurai, Phys. Rev. Lett. $11,48(1963)$; S.L.

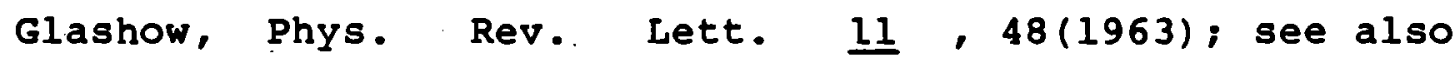
Dashen and Sharp in Reference 1.

3. See, for example, P.J. O'Donnell, Rev. of Mod. Phys. $\underline{53}, 4(1981)$

4. S. Meshkov, C.A. Levinson, and H.S. Lipkin, Phys. Rev. Lett. $10,361(1963 ;$ B.T. Feld, Models of Elementary Particles ; (Blaisdell, Waltham,1969); J. Bernstein, Elementary Particles and Their Currents. (Freeman, San Francisco, 1968).

5. P.J. O'Donnell, Canadian Joụnal of Physics, $\underline{55}$, 1301(1977); see also M. Gell-Mann and F. Zachariasen in Reference 1 . 
6. See M. Gell-Mann, D. Sharp, and W.G. Wagner in Reference 1 .

7. Particle Data Group, Rev. Mod. Phys. 52, S1(1980).

8. See, for instance, $\dot{M}$. Gell-Mann, Phys. Lett. $\underline{8}$, 214(1964); G. Zweig, CERN Rep. 8419/TH (1964); G. Zweig, Symmetries in Elementary Particle physics, ed. A. Zichichi, (Academic Press, New York) p. 192 (1965); J.J. Kokkedee, The Quark Model, (W.A. Benjamin, Inc., New York, 1969); A.W. Hardy and D.B. Lichtenberg, Rep. Prog. Phys. $41,1707(1978)$.

9. J. Sucher, Rep. Prog. Phys. 41, 1781(1978).

10. C. Becchi and G. Morpurgo, Phys. Rev. 140 , 687B (1965); Y. Anisovitch et al., Phys. Lett. 16 , $194(1965) ;$ W. Thirring, Phys. Lett. $16,335(1965) ;$ L. Soliev, Phys. Lett. $16,345(1965)$.

11. See P.J. O'Donnell(Reference 3) for a more complete development.

12. S. Ono, Prog. Theo. Phys. 50, 589(1973); s. Ono, Nuovo Cimento Lett. 14 , 569(1975); N. Isgur, Phys. Rev. Lett. $36,1262(1976)$.

13. A.H. Rosenfeld, A. Barbaro-Gualtieri, W.H. Barkes, P. Bastien, J. Kirz, and M. Roos, Rev. Mod. Phys. $\underline{36}$, $977(1964)$. 
14. T. Ohshima, Phys. Rev. D $\underline{22}, 707(1980)$.

15. B. Gobbi et al., Phys. Rev. Lett. $\underline{33}, 1450(1974)$; B. Gobbi et al., Phys. Rev. Lett. $\underline{37}$, 1439(1976).

16. A.N. Kamal and G.L. Kane, Phys. Rev. Lett. 43,551 .

17. T. Jensen, Ph. D. Thesis, University of Rochester Report UR-747; D. Berg et a!̣., Phys. Rev. Lett. 44 , 706(1980); T. Jensen et al... University of Rochester Report UR-826 (to be published in Physical Review D).

18. R.H. Hackman, N.G. Deshpande, D.A. Dicus, and V.L. Teplitz, Phys. Rev. D 18, 2537(1978); M. Frank, P.J. O'Donnell and B. Wong, Z. Phys. C $I, 277(1981)$.

19.. J. Bjorken and.S. Drell, Relativistic Quantum Mechanics , (McGraw-Hill, Inc., 1964).

20. G. Fidecano et al., Phys. Lett. $\underline{23}, 163(1966)$.

21. Y. Eisenberg et al., Phys. Rev. Lett. $25,764(1970)$.

22. H. Primakoff, Phys. Rev. 81, 899(1951).

23. G. Bellettini et al., Nuovo Cimento 40A, 1139(1965); G. Bellettini et al., Nuovo Cimento 66A, 243(1970); V.I. Kryskin et al., soviet Physics JETP $\underline{30}, 1037(1970)$; C. Bemporad et al., Phys. Lett. 25B , 380 (1967).

24. See, for instance, T. Ferbel, Proceedings of the lst 
Workshop on Ultra-Relativistic Nuclear Collisions, LBL-8957, 1979 .

25. See, for example, V.D. Barger and D.B. Cline, Phenomenological Theories of High Energy Scattering, (W.A. Benjamin, Inc., New York, 1969).

26. L. Stodolsky, Phys. Rev. $144,1145(1966)$.

27. K. Gottfried and J.D. Jackson, Nuovo Cimento 33 , $309(1964)$.

28. G. Berlad et al., Ann. of Phys. $\underline{75}, 461(1973) ;$ M. Gourdin, Nuclear Physics $\underline{32 B}, 415(1971) ; A$. Halprin, C.M. Andersen, and H. Primakoff, Phys. Rev. $\underline{152}$, 1295(1966); N. Jurisic and L. Stodolsky, Phys. Rev. D3,724(1971). For an excellent review, see also L. Strawczynski, Ph. D. Thesis, University of Rochester Report UR-475(1974).

29. See A. Halprin et al. and $\mathrm{N}$. Jurisic et al. in Reference 28 .

30. H. Harrari, Phys. Rev. Lett. 26, 1400(1971); see also L. Stodolsky in Reference 26.

31. See the M-I User's Guide, S. Ecklund, Fermilab Report TM-743.2833, May 1977 .

32. W.F. Baker et al., "Production of $\pi^{ \pm}, \mathrm{K}^{ \pm}, \mathrm{p}$ and $\overline{\mathrm{p}}$ by 400 GeV Protons," Fermilab Report-78/79-EXP. 
33. A. Jonckheere et al., Nucl. Instr. and Meth. 180 , $25(1981)$

34. For a more detailed description of the drift chamber system, see T. Jensen, Ph. D. Thesis, Reference 17.

35. P. Koehler et al., Fermilab Proposal E272(Revised), "Proposal to Measure Coherent Dissociation of $\pi^{-}, \mathrm{K}^{-}$, and $\bar{p}$ into Two-Body Systems at Fermilab Energies," May (1975).

36. W.S. Willis and V. Radeka, Nucl. Instr, and Meth. 120 , 221(1974). For more information on the construction and operation of liquid argon calorimeters, see also G. Knies an D. Neuffer, Nucl. Instr. and Meth. $120,1(1974) ; \mathrm{J}$. Engler et al., Nucl. ilnstr. and Meth., $120,157(1974) ;$ D. Hitlin et al., Nucl. Instr. and Meth., $137,225(1976) ; C$. Cerri and F. Sergiampietri, Nucl. Instr. and Meth. 141,207(1977); J.H. Cobb et al., Nucl. Instr. and Meth. 158, 93(1979); C. Nelson et al., Fermilab-Pub-82/41-EXP (submitted to Nucl. Inst. and Meth.)

37. See J. Engler et al. in Reference 36.

38. See W.J. Willis and V. Radeka, J. Engler et al., D. Hitlin et al., and C.W. Fabjan et al., in Reference 36.

39. For a more detailed description of the LAC electronics see T.F. Droege, F. Lobkowicz, and Y. Fukushima, 
Fermilab Report TM-746 2500.000, October(1977). See also c. Nelson et al. in Reference 36 .

40. A review of trigger processor use at Fermilab is given by T. Nash, Fermilab Report-81/39 2100.000, October(1978).

41. C. Kerns, Fermilab Report TM-819 2510.000, (October 1978).

42. A. Brenner et al., Fermilab Report TM-537 2340.000, (December 1974).

43. C. Chandlee, Ph.D. Thesis, University of Rochester (unpublished).

44. S. Cihangir, Ph.D. Thesis, University of Rochester (unpublished).

45. An excellent discussion of calorimeter resolution can be found in a Tristan workshop review by Seigi Iwata, "Calorimeters (Total Absorption Detectors) for High Energy Experiments at Accelerators," Department of Physics, Nagoya University Report DRNu-3-79, (February 1979).

46. B. Rossi, High Energy Particles, (Prentice-Hall, Inc., 1952)

47. A.S. Carroll et al., Phys. Rev. Lett. 33, 932(1974). 48. For more details on the level of muon contamination, see 
J. Huston, "Muon Contamination in E272," University of Rochester Internal Report.

49. A.S. Carroll et al., Phys. Lett. $80 \mathrm{~B}, 319(1979)$.

50. For $\mathrm{z}>20$, this formula is accurate to 18 or better for photons of energy $10 \mathrm{GeV}$ or greater. See $\mathbf{s}$. Iwata (Reference 45), p. 10.

51. R.T. Deck, Phys. Rev. Lett. $13,169(1964)$.

52. J.D. Jackson, Nuovo Cimento 34,1644 (1964).

53. R.L. Walker, Phys. Rev. $182,1729(1969)$.

54. Particle Data Group, Phys. Lett. 111B, 1(1982). The Particle Data Group averages three experiments( see Footnotes 55 and 56 ) to obtain the charged $\rho$ mass and width. The values they obtain are $m_{\rho}=(766.8 \pm 1.4) \mathrm{MeV}$ and $\Gamma_{\rho}=(147.8 \pm 3.7) \quad \mathrm{MeV}$. By considering measurements involving the neutral $\rho$, they conclude that the mass of the $\rho$ is $(769 \pm 3) \mathrm{MeV}$. They also note that an estimate of the mass of the $\rho$ can be obtained from a su(4) generalization of the Gell-Mann-Okubo mass formula. In the limit of ideal mixing it can be written

$$
\rho=\frac{2\left(\Psi K^{*}-\phi D^{\star}\right)+\omega\left(D^{*}-K^{*}\right)}{2(\Psi-\phi)-\left(D^{\star}-K^{\star}\right)}
$$


Substituting in the values for the masses of the mesons on the right-hand side(which can be determined to much better accuracy than the $\rho)$, we obtain $m_{\rho}=(768 \pm 2) \mathrm{MeV}$.

55. J. Pisut and M. Roos, Nucl. Phys. B6 ,325(1968); L. Byerly et al., Phys. Rev. D $\underline{7}, 637$ (1973).

56. R.L. Eisner et al., Phys. Rev. 164, 1699 (1967). 57. C. Bemporad et al., Nucl. Phys. B51, 1(1973).

58. K.J. Kolbig and B. Margolis, Nucl. Phys. B6 ,85(1968); G. Faldt et al., Nucl. Phys. B4l 125 (1972); G. Faldt, Nucl. Phys. B43, 591(1972). See also T. Ohshima, "Numerical Calculation for Coherent $\gamma \pi+\rho$ Produçtion," University of Rochester Internal Report.

59. The $\pi^{+}$-nucleon cross section, $24.5 \mathrm{mb}$, was obtained from the Particle Data Tables (Reference 7 ). The value of $\alpha$ used, 0.053, was obtained from I. Fajardo et al., Phys. Rev. D $24,46(1981)$. The value of $\sigma_{\pi N}$ or $\alpha$ used has only. a small effect on the fitted value for $\Gamma\left(\rho^{+}+\pi^{+} \gamma\right)$. For example, setting $\sigma_{\pi N}$ to 0 results in a change in the resultant value of $\Gamma$ of only 1.58 .

60. D.J. Crennel et al., Phys. Rev. Lett. $27,1674(1971)$; J. Bartsch et al., Nucl. Phys. B46, 46(1972). See also B. Gobbi et al., Reference 15, and T. Jensen et al., in Reference 17. 
61. See T. Jensen et al., Reference 17 . The results contained in this paper supersede all previous analysis by our group on the radiative width of the $p^{-}$.

62. For detailed discussions on electron drift in liquid argon and the effect of impurities, see D.W. Swan, Proc. Phys. Soc. $\underline{83}, 659(1964) ;$ L.S. Miller, S. Howe, and W.E. Spear, Phys. Rev. $166,871(1968) ;$ E. Shibamura et al., Nucl. Instr. and Meth. $131,249(1975)$. See also J. Engler et al., in Reference 36 .

63. See J.H. Cobb et al. in Reference 36 . 
Appendix A

Charge collection in the LAC

Electrons produced by ionization in the liquid argon gaps were collected by the readout strips on the copper-clad G-10 boards. The amount of collectible charge produced can be written

$$
Q=f e \frac{E}{2 \cdot 26.4 e v}
$$

where $f$ is the fraction of energy deposited in the liquid argon (about 0.16 in this case), $\mathbf{E}$ is the energy of the incident photon or electron, and $26.4 \mathrm{ev}$ is the lonization potential of argon. Only half of the produced charge can be collected in a reasonable time due to the low mobility of the positive ions. That is, half of the electron charge is frozen in order to image the positive ion charge. Figure $58 a$ shows a schematic representation of one cell of the LAC and Figures $58 \mathrm{~b}$ and $58 \mathrm{c}$ show the current and charge waveforms for the case of uniform ionization. Perhaps the most salient feature of the charge waveform is that 758 of the charge is collected in half of the total drift time. At high electric fields, the total drift time can be parameterized as [62] 
a)

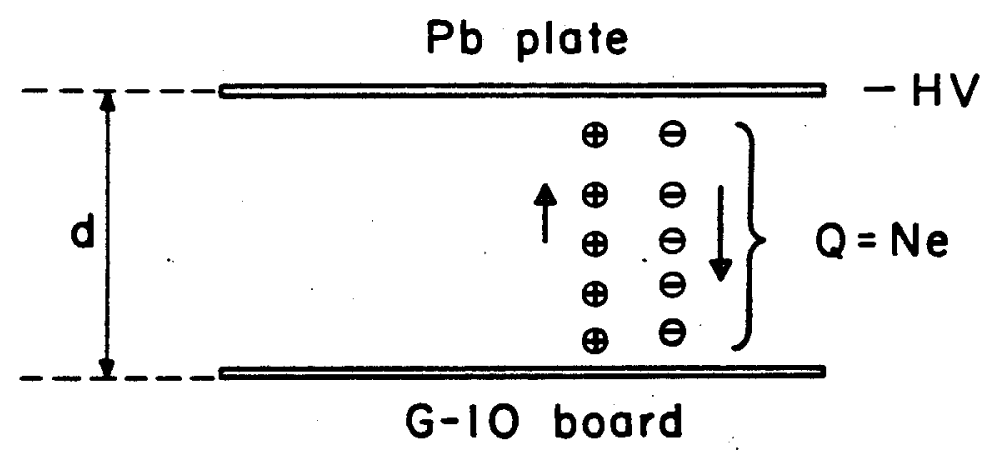

b)

c)
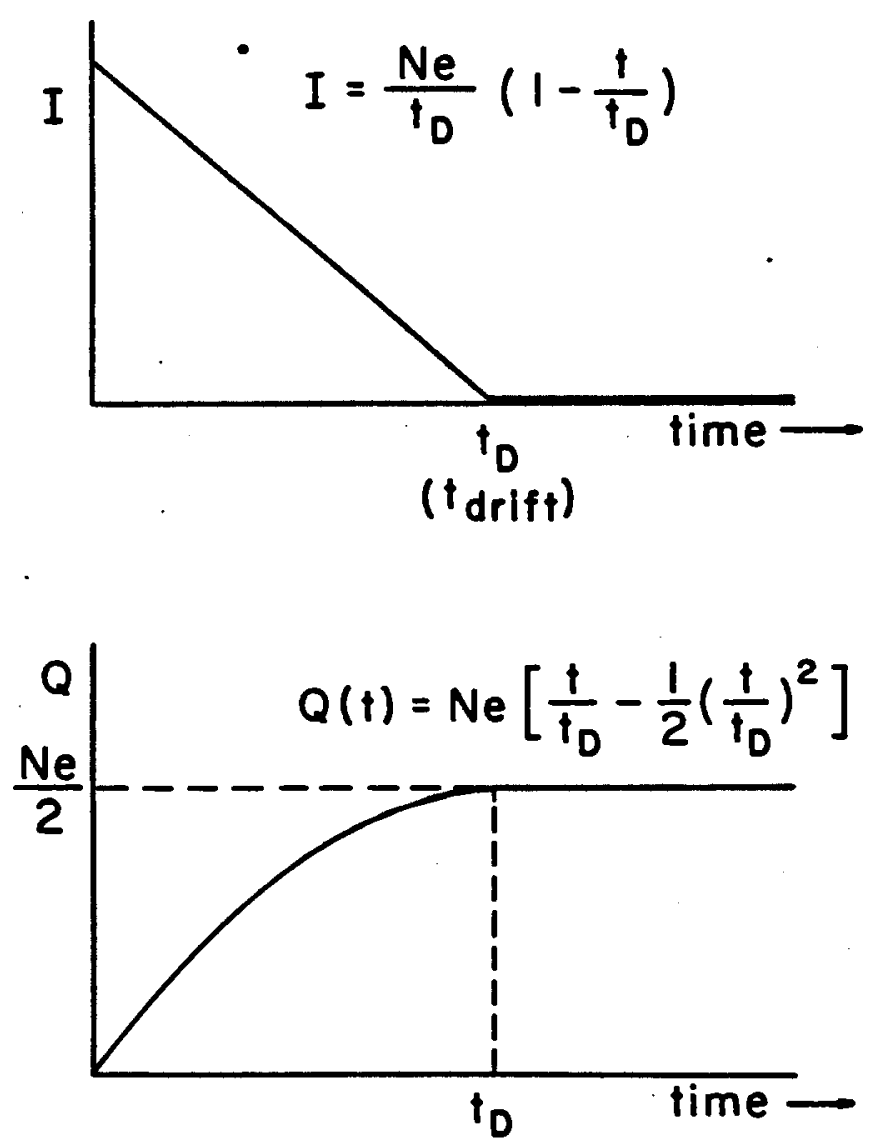

Figure 58 


$$
t_{\text {drift }}=\frac{\text { gap width }}{2.1 \times 10^{4}[\mathrm{E}(\mathrm{v} / \mathrm{cm})]^{1 / 3}}
$$

For a gap width of $0.2 \mathrm{~cm}$ and an electric field of 12.5 $\mathrm{kV} / \mathrm{cm}$, the drift time is approximately 400 nanoseconds.

Figure 59 shows the ratio of the charge collected to the total charge expected from Equation (A.I) as a function of electric field. The charge collection curve was taken during the $50 \mathrm{GeV}$ calibration run. At the operating point of the detector, only about 418 of the charge expected was actually seen. In a paper reporting results from a liquid argon calorimeter at the ISR, Cobb et al [63] state that Equation (A.I) is correct for incident muons but gives a value approximately 308 too high for incident electrons. The additional loss for heavily lonizing showers is attributed to transition effects between the argon and lead.

Part of the remaining discrepancy can be attributed to recombination of the electron-ion pairs. Studies using a B source have found the fraction of electrons that escape recombination to be

$$
\mathrm{f}=\frac{1}{1+\mathrm{K} / \mathrm{E}} \quad \mathrm{K}=1.1+0.2 \mathrm{kV} / \mathrm{cm}
$$




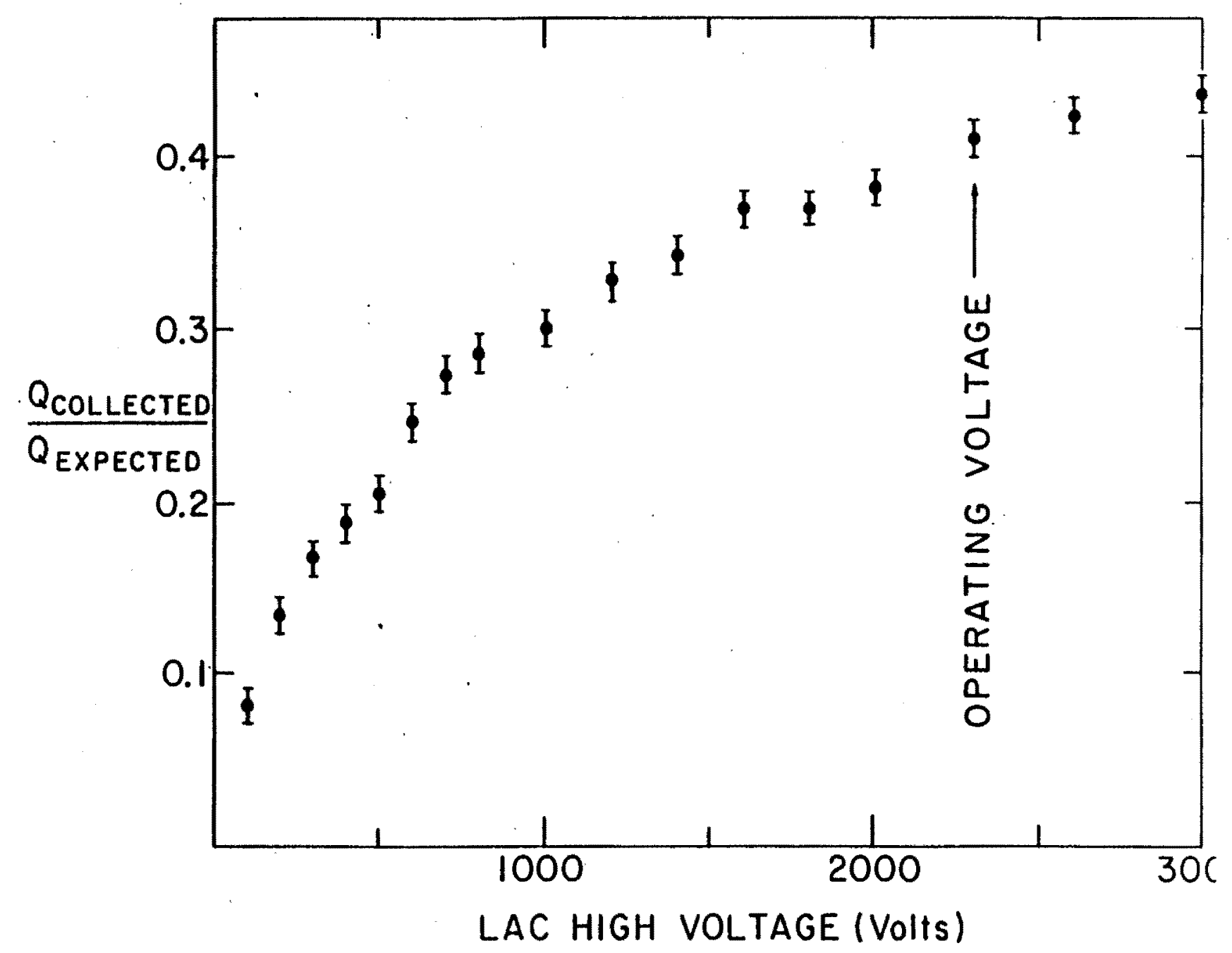

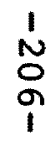

Figure 59 
For a field of $12.5 \mathrm{kV} / \mathrm{cm}, f=.92$. Due to transition and recombination effects, then, only 548 of the charge given by Equation (A.I) can be expected to be collected. This is still far greater than the 418 shown in Figure 59.

The mean free path of an electron in liquid argon in the presence of oxygen can be written

$$
s=0.12-\left[\frac{\mathrm{E}-\mathrm{cm} \mathrm{O}^{2}, \mathrm{ppm}}{\mathrm{p}}\right]
$$

The charge collected for a mean free path $\mathbf{s}$ can be written

$$
Q=Q_{o} f-\frac{s}{d}\left[1-e^{-d / s}\right]
$$

where $f$, again, is the recombination factor and $d$ is the gap width. If the remaining charge loss was attributed to the presence of oxygen, then the argon in the LAC would have had to contain about 6 parts per million of oxygen. (For E=12.5 $\mathrm{kV} / \mathrm{cm}$ and $\mathrm{p}=6.0 \mathrm{ppm}, \mathrm{s}=.25 \mathrm{~cm}$.$) As mentioned in Chapter II,$ the argon was purified before liquefication. The argon at the output of the purification system was monitored and found to contain less than 1 ppm of oxygen. We thus feel confident that our oxygen contamination was negligible and that the loss in pulse height must be attributed to some other 
electronegative impurity. During the course of our run the charge collected was found to increase by approximately $30 \%$. The increase was approximately linear with time. This increase in charge collection efficiency was noted by a corresponding increase in the observed $\pi^{\circ}$ mass from $\mathrm{K}^{+} \rightarrow \pi^{+} \pi^{\circ}$ decays. Since the amplifier gains and the LAC high voltage were monitored and found to remain constant, the increase in the $\pi^{\circ}$ mass must have been due to an increase in the collected charge. We have attributed the improvement in electron transport efficiency to a "freezing out" of some electronegative impurity during the course of the run. Figure 33 shows the increase with time of the collected charge. The roughly linear slope is consistent with a constant "freezing out" of an electronegative impurity. At the end of the run the level of charge collection was close to that predicted by Equation (A.I) with the transition and recombination effects taken into account. 
Appendix $B$

$\mathrm{P}_{t}$ Resolution from $\mathrm{K}^{+} \rightarrow \pi^{+} \pi^{\circ}$ Decays

A measure of the resolution of the spectrometer in $t$ or $p_{t}$ was provided by the decay $\mathrm{k}^{+}+\pi^{+} \pi^{\circ}$. A large sample of $\mathrm{K}^{+}+\pi^{+} \pi^{\circ}$ events were collected for each target over the course of the run. A derivation of the expected $t$ distribution for these decays is given below and some of the assumptions that have gone into this derivation are discussed.

The assumption was made that the measured transverse momentum $\vec{\nabla}_{t}$ had a gaussian distribution that was centered at the true transverse momentum $\tilde{\vec{p}}_{t}$ with variances in the $x$ and $y$ directions of $\sigma_{\mathrm{p}_{t x}}{ }^{2}$ and $\sigma_{\mathrm{p}_{t y}}{ }^{2}$. It was further assumed that $\sigma_{p_{t x}}=\sigma_{p_{t y}}$, that is, that the spectrometer had equal resolution in the horizontal and vertical directions and also that $\sigma_{p_{\text {tx }}}$ and $\sigma_{p_{t y}}$ were uncorrelated. The resolution function expressed in terms of transverse momentum can be written

$$
R\left(\overrightarrow{\vec{p}}_{t}, \tilde{\vec{p}}_{t}\right)=\frac{1}{2 \pi \sigma^{2}} e^{-\left[\left(\overrightarrow{\mathrm{p}}_{t}-\tilde{\vec{p}}_{t}\right)^{2} / 2 \sigma^{2}\right]}
$$

so the expected distribution of events in $p_{t}^{2}$ is 


$$
\frac{d \sigma}{d t}\left(p_{t}{ }^{2}\right)=\int R\left(\vec{p}_{t}, \widetilde{\vec{D}}_{t}\right) \frac{d \sigma}{d \tilde{t}}\left(\tilde{p}_{t}^{2}\right) d^{2} \tilde{\vec{p}}_{t}
$$

Transforming from $\mathrm{p}_{t}{ }^{2}$ to $t$, the above equation becomes

$$
\begin{gathered}
\frac{d \sigma}{d t}(t)=\frac{\cos \theta}{2 \sigma^{2}} \int d \tilde{t} \quad e^{-\left[\left(p_{t}-\widetilde{p}_{t}\right)^{2} / 2 \sigma^{2}\right]} e^{-\left[2 p_{t} \widetilde{p}_{t} / 2 \sigma^{2}\right]} \\
I_{0}\left(\frac{2 p_{t} \widetilde{p}_{t}}{2 \sigma^{2}}\right) \cos \tilde{\theta} \frac{d \sigma}{d \tilde{t}}(\tilde{t})
\end{gathered}
$$

Since the process is a decay

$$
\frac{d \sigma}{d \tilde{t}}(\tilde{t})=\frac{d \sigma}{d \tilde{t}}(0) \delta(\tilde{t})
$$

and since $\tilde{t}=0, \tilde{p}_{t}=0$ and $\cos \tilde{\theta}=1$. Substituting, we have

$$
\begin{aligned}
\frac{d \sigma}{d t}(t) & =\frac{1}{2 \sigma^{2}} \frac{d \sigma}{d t}(0) e^{-p_{t}^{2} / 2 \sigma^{2}} \\
& =\frac{1}{2 \sigma^{2}} \frac{d \sigma}{d t}(0) e^{-t / 2 \sigma^{2}}
\end{aligned}
$$

The observed event distribution can thus be parameterized

$$
\text { Events }(t)=E_{0} e^{-t / 2 \sigma^{2}}
$$


The above function was used in the fits of the observed $\mathrm{K}^{+}$ decay $t$ distributions, to obtain the resolution $\sigma$. 
